This document is downloaded from DR-NTU (https://dr.ntu.edu.sg) Nanyang Technological University, Singapore.

Study on muscle remodeling using in vivo microscopy of drosophila metamorphosis and quantitative image analysis

Kuleesha

2018

http://hdl.handle.net/10356/73192

https://doi.org/10.32657/10356/73192 


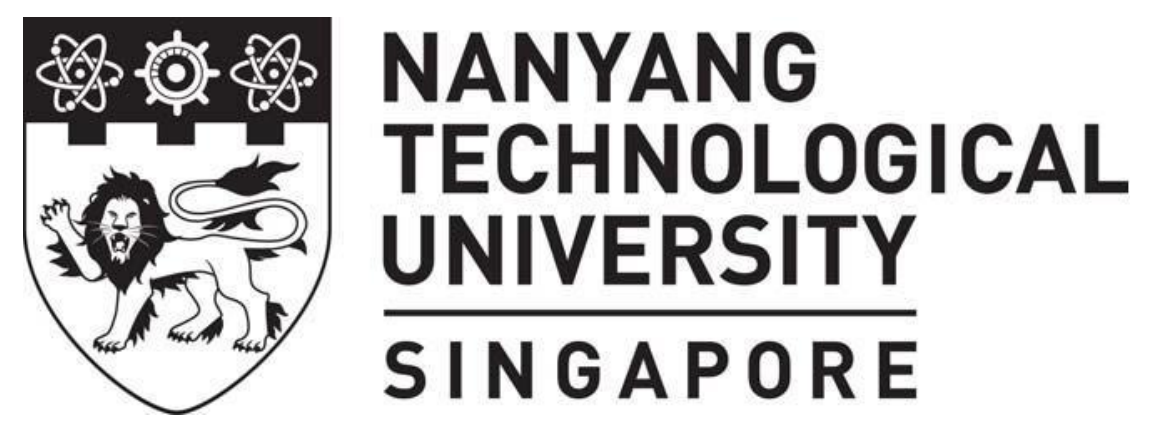

STUDY ON MUSCLE REMODELING USING IN VIVO MICROSCOPY OF DROSOPHILA METAMORPHOSIS AND QUANTITATIVE IMAGE ANALYSIS

KULEESHA

SCHOOL OF COMPUTER SCIENCE AND ENGINEERING 2018 


\section{STUDY ON MUSCLE REMODELING USING IN VIVO MICROSCOPY OF DROSOPHILA METAMORPHOSIS AND QUANTITATIVE IMAGE ANALYSIS}

\section{KULEESHA}

SCHOOL OF COMPUTER SCIENCE AND ENGINEERING

A thesis submitted to the Nanyang

Technological University in partial fulfilment of

the requirement for the degree of Doctor of

Philosophy

2018 


\section{Acknowledgements}

I thank my supervisors Associate Professor Lin Feng and Dr Martin Wasser for giving me valuable advice for this project. I would like to thank Wee Choo for her help in fly work and imaging, Wee Thong for the segmentation evaluation tool and advice on the FMAj tool design. I thank Dr Lee Hwee Kuan for providing academic advice on my research. I thank the IMCB (ACC and SC labs) for the use of Drosophila facilities.

I thank Nanyang Technological University and Bioinformatics Institute, Agency for Science, Technology and Research (A*STAR) for funding the research. I acknowledge the Bloomington Drosophila Stock center for providing fly stocks. I thank the TRiP at Harvard Medical School (NIH/NIGMS R01-GM084947) for providing transgenic RNAi fly stocks. 


\section{Table of Contents}

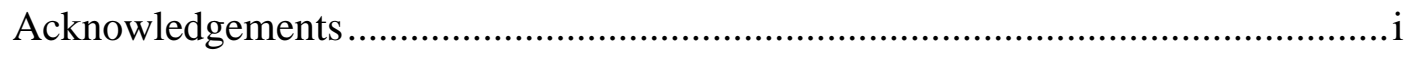

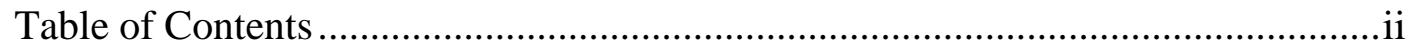

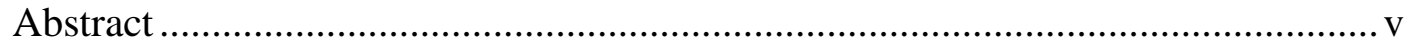

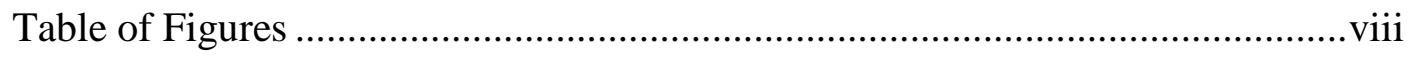

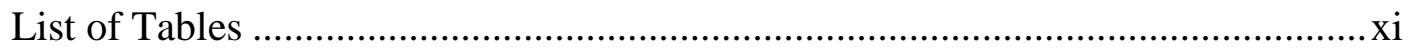

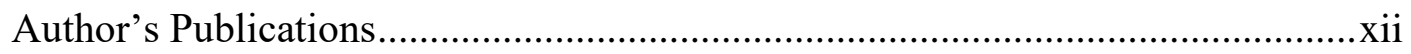

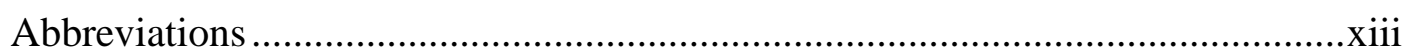

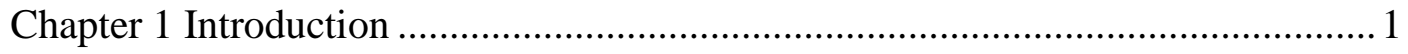

1.1 Skeletal Muscle Biology .................................................................. 1

1.1.1 Significance of muscle mass change ................................................ 2

1.1.2 Molecular mechanism of muscle mass control......................................... 3

1.2 Drosophila as a Model to Study Muscle Mass Change ................................ 5

1.2.1 Muscle development in Drosophila .................................................. 5

1.2.2 Signalling pathways regulating metamorphosis .................................. 9

1.2.3 Advantages of Drosophila for genetic studies ................................... 9

1.3 Nuclear Positioning in Muscles.................................................................. 11

1.4 Imaging as a Tool for Genetic Analysis ................................................... 12

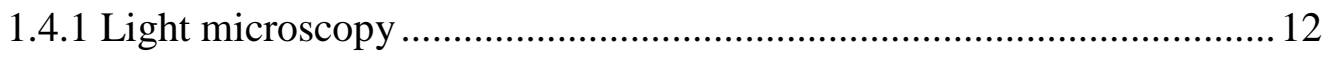

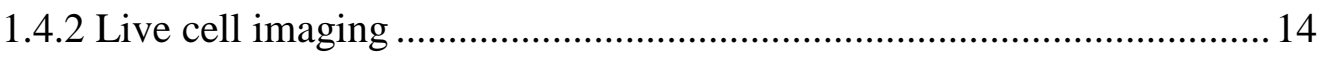

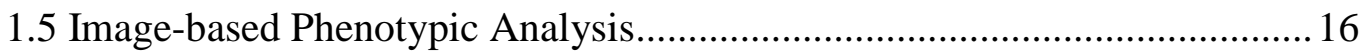

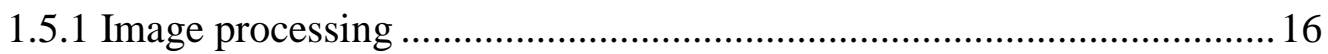

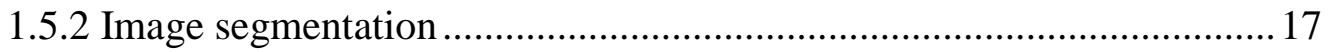

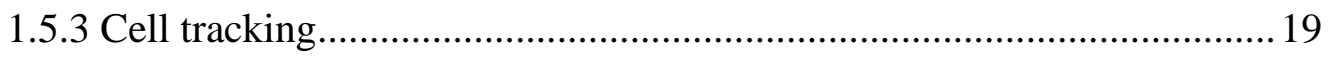

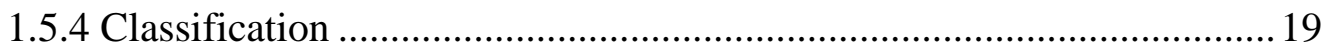

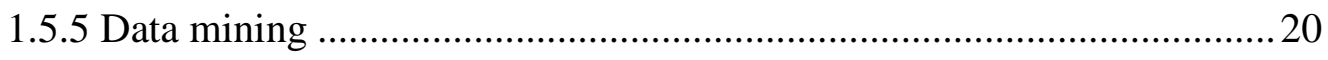

1.6 Related Work on Postembryonic Muscle Development ..............................220

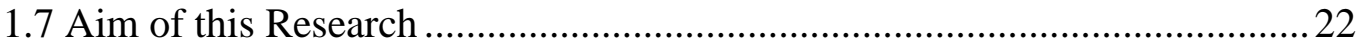


1.8 Organization of the Thesis .23

Chapter 2 Experimental Work on Genetic Perturbation and Imaging Protocol ..... 25

2.1 Targeted Misexpression using the GAL4/UAS System .26

2.1.1 RNA Interference. 27

2.2 Targeted Genetic Perturbation in Muscles .28

2.2.1 Drosophila Strains .28

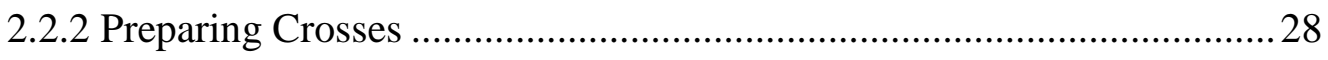

2.2.3 Specimen preparation for imaging ..................................................2 29

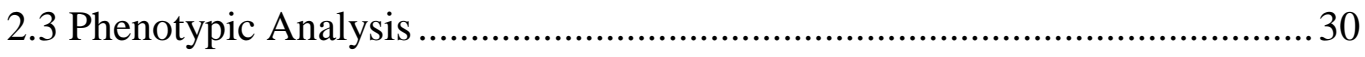

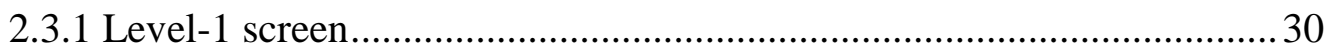

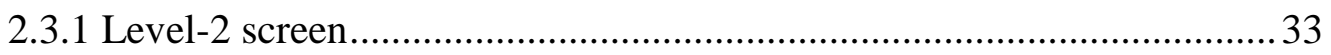

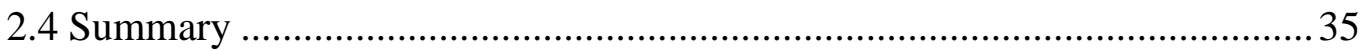

Chapter 3 FMAj for Time Series Analysis of Drosophila Muscles....................... 36

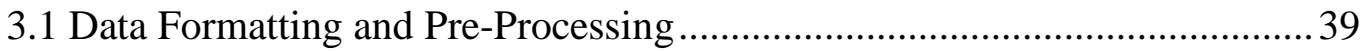

3.2 Image Analysis Workflow of the FMAj Tool ............................................ 39

3.2.1 Annotation of time-series images .................................................... 41

3.2.2 Segmentation and feature extraction .............................................. 41

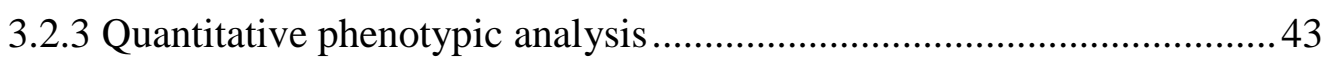

3.3 Relational Database Management ........................................................ 45

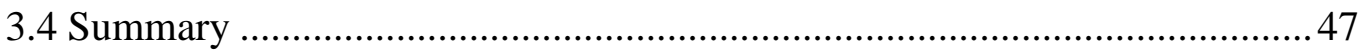

Chapter 4 Time Series Segmentation of Muscles in an Environment containing Sarcolytes .48

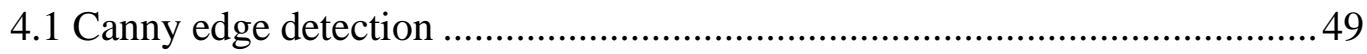

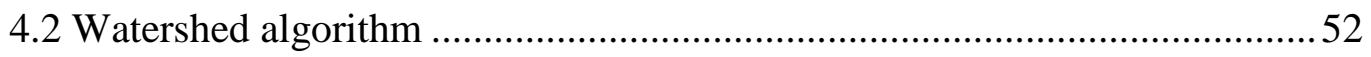

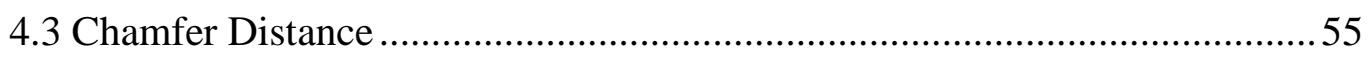

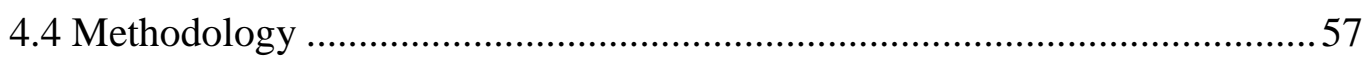

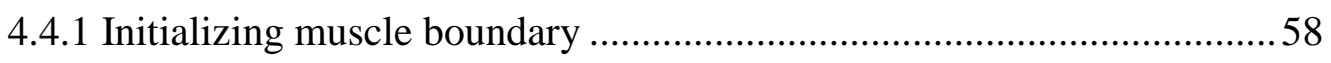

4.4.2 Edge confidence map generation using watershed segmentation .......59 
4.4.3 Region classification algorithm 60

4.5 Muscle Morphological Features.... .63

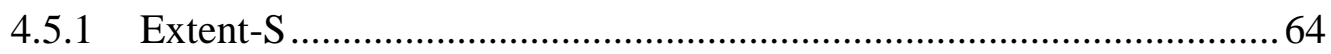

4.5.2 Polar histogram based morphological feature ....................................65

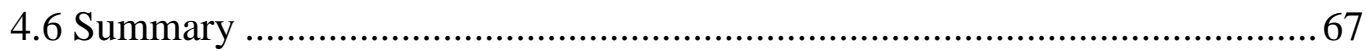

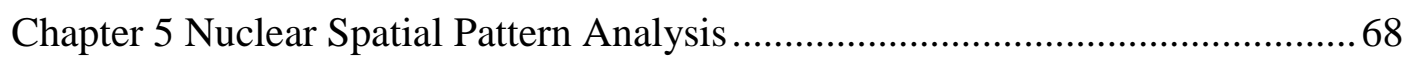

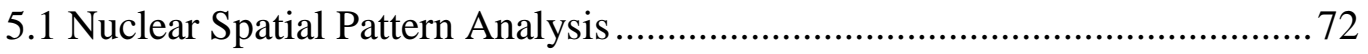

5.1.1 Nuclear region extraction inside persistent muscles............................ 73

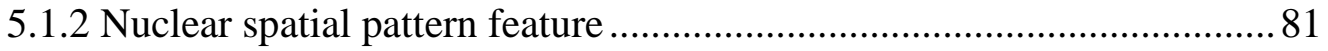

Rearrangement of nuclei in straightened muscle cell .................................. 81

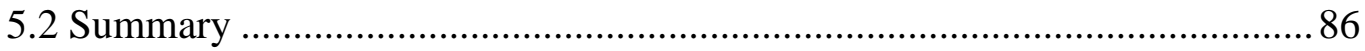

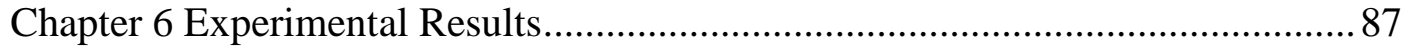

6.1 Testing and Validation of Muscle Segmentation ...................................... 87

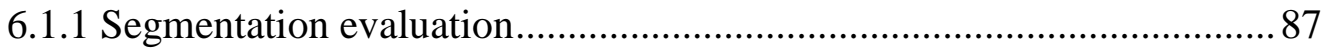

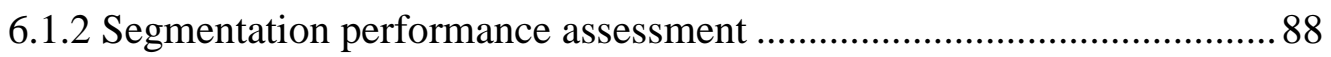

6.2 Evaluation of Nuclear Segmentation and Classification ............................. 91

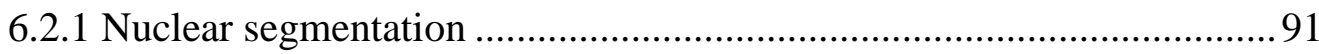

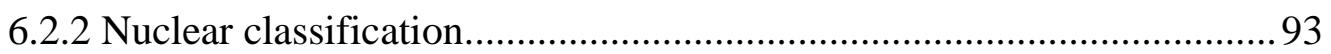

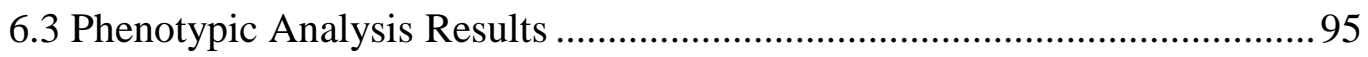

6.3.1 Morphological analysis of muscle cells........................................... 104

6.3.2 Nuclear spatial pattern analysis results.............................................. 107

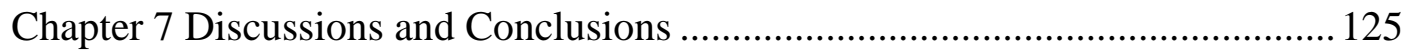

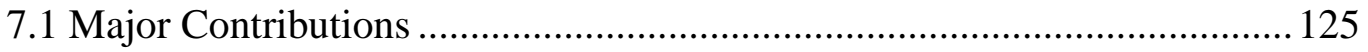

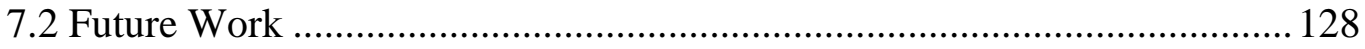

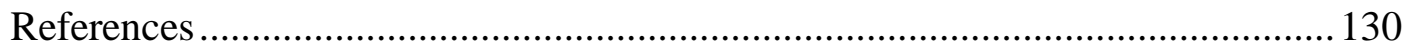




\section{Abstract}

Skeletal muscle cells control most of the physical movements in our body, from simple actions such as smiling or frowning to critical movement of the diaphragm for breathing. Maintenance of muscle mass and strength is essential for physical functions and mobility. The human body suffers from various muscular ailments ranging from the most common age related muscle loss to fatal muscular dystrophy. Advances in the understanding of signaling pathways involved in muscle mass control would facilitate the identification of pharmacological cure for muscle diseases.

The association between skeletal muscle mass and muscular disorder has been studied extensively in various organisms. However, only a few of them have used in vivo imaging to understand the development of the muscle particularly in insects such as Drosophila melanogaster and moth where the muscles undergo extreme remodeling during metamorphosis. In Drosophila melanogaster, larval muscles undergo two different developmental fates during metamorphosis; one population is removed by cell death, while the other persistent subset undergoes morphological remodeling and survives to adulthood. Thanks to the ability to perform live imaging of muscle development in transparent pupae and the power of genetics, metamorphosis in Drosophila can be used as a model to study the regulation of skeletal muscle mass. In this thesis, my objective is to explore in vivo imaging of Drosophila metamorphosis as a model to study muscle mass regulation at large scale using quantitative image analysis and discover new insights into gene functions in muscles which could not be found using traditional endpoint assays. We found new phenotypes for many genes by quantifying the morphological changes in persistent muscles and myonuclear distribution using my custom tool FMAj. A detailed analysis of muscle phenotypes in four autophagy genes, that is $\operatorname{Atg} 5, \operatorname{Atg} 9, \operatorname{Atg} 12$ and $\operatorname{Atg} 18$ revealed that autophagy acts transiently to promote autophagy. Additionally, we found that the loss of autophagy due to silencing of Atg9 and Atg18 enhanced the anti-polar migration of nuclei during mid-pupal development and decreased polar migration toward the latepupal development. Phenotypic analysis revealed that SNF1A and Grunge are involved in regulating the cell death of Drosophila abdominal muscles during 
metamorphosis.

In this study, our team characterized the phenotypic changes in Drosophila muscle cells caused by genetic perturbation during metamorphosis using time lapses in vivo imaging. A combination of RNAi and UAS-GAL4 system was used for muscle specific gene silencing and fluorophore expression. With the availability of advanced fluorescence microscopes and a reporter line with two markers, we acquired time lapse images of muscles and myonuclei. However, 3D time lapse imaging of Drosophila metamorphosis for 4-5 days generated large number of images (10-15 GB per sample) which is a bottleneck for the quantitative phenotypic analysis.

In order to quantify the phenotypic changes in large number of images, I designed a custom tool, Fly Muscle Analysis in Java (FMAj), integrated with a relational database to perform various tasks such as annotation, segmentation, feature generation and statistical analysis. The integration of multiple tasks enhances productivity as the alternative export of data and the manual processing in a spreadsheet program would be much more time-consuming. My first objective was to study the morphological changes in muscle cells during metamorphosis for which muscle cell boundaries were required. However unlike other cells like hela, muscle cells in Drosophila pupae are surrounded by dead sequestered muscles (debris), which makes it difficult to extract muscle boundary with high accuracy. Therefore, I designed a muscle segmentation technique which is capable of differentiating muscle and debris regions. I combined watershed based region classification with chamfer distance based shape feature and an edge confidence feature to obtain muscle boundary in time series stack. The shape information extracted from muscle boundary at previous time points restricts the muscle boundary in next time point from moving to debris. Comparative studies show that the accuracy of my method is better than other segmentation techniques.

I also performed nuclear spatial pattern analysis to understand the relationship between muscle mass change and the distribution of nuclei inside muscles. After nuclear segmentation, the regions were classified into two types of nuclei i.e. the nuclei inside muscle cells (internal nuclei) and the nuclei present inside debris produced during histolysis of muscle cell (external nuclei). I designed a tracking based nuclei classification algorithm which exploits the difference in the motion of internal and external nuclei. Comparative analysis show that my nuclear classification 
technique performs better than other techniques. I designed new nuclear spatial pattern features, each catering to a specific type of nuclei migration pattern.

In conclusion, the work in this thesis has been able to justify Drosophila melanogaster as a good model to study metamorphosis and to find new insights into the genes associated with muscle mass change. My tool provides additional support by providing an image processing platform to confirm the findings quantitatively. 


\section{Table of Figures}

Figure 1.a) Anatomy of skeletal muscle b) Effect of muscle contraction in sarcomere. 2 Figure 2.Significance of muscle mass. 3

Figure 3. Regulation of protein metabolism by two pathways: PI3K/AKT signaling and autophagy mechanism...... .5

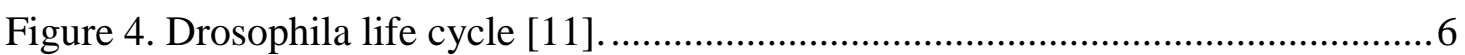

Figure 5. Muscle development in Drosophila at embryonic, larval and pupal stage......7

Figure 6. Workflow of the muscle analysis framework and contributions of this thesis.

Figure 7. Tissue specific gene silencing by combining UAS-GAL4 system and RNAi approach. .26

Figure 8. Muscle specific expression of two fluorescent proteins using UAS-GAL4 system.

Figure 9. Muscle phenotype at different stages of development during metamorphosis.

Figure 10. a) Macro zoom fluorescence microscope b) Line scanning Zeiss LSM 5 Live microscope. .34

Figure 11. Pipeline for quantitative time series phenotypic analysis of muscles. ….....38

Figure 12. Screenshot of the FMAj user interface. 40

Figure 13. Quantitative phenotypic analysis using FMAj. .44

Figure 14. Screenshot of an FMAj application to view and compare the muscle contours between two samples. .45

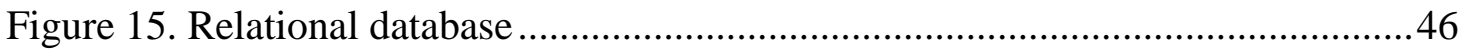

Figure 16. Sequestered muscle debris attached to the muscle cells .............................49

Figure 17. Comparison of different edge detection techniques ..................................51

Figure 18. Muscle boundary extraction using canny detector. ..................................52

Figure 19. Watershed regions generated at different values of threshold $h$.................53

Figure 20. Comparison between the performances of morphological watershed (b1, b2) and $\mathrm{N}$-cut $(\mathrm{c} 1, \mathrm{c} 2)$ segmentation on muscle image. Yellow arrowhead shows the edge which $\mathrm{N}$-cut was not able to identify. .54 
Figure 21. Oriented chamfer distance .56

Figure 22. Schematic overview of muscle segmentation. .58

Figure 23. The figure shows the results of watershed segmentation and the corresponding edge confidence map.

Figure 24. Region classification and its subsections.

Figure 25. (A) Bounding box of a muscle cell. (B) Bounding box of (A) muscle cell if it was not bent.

Figure 26. Comparison between the values of extent and extent-S in two different muscle cells.

Figure 27. Polar histogram based derivation of elongation feature. .66

Figure 28 Schematic diagram explaining different stages of nuclear localization....... 70

Figure 29 Workflow of the nuclear spatial pattern analysis pipeline. .72

Figure 30 a) Example of internal and external nuclei. b) Examples of nuclei clustering which complicates the tracking of nuclei. .73

Figure 31. Results of different stages of myonuclear segmentation...........................75

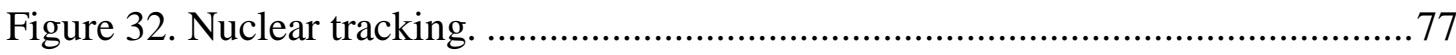

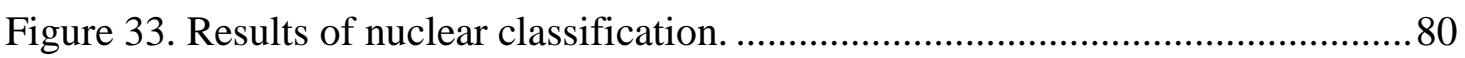

Figure 34. Rearrangement of nuclei in straightened muscle cell for comparing nuclei from different samples. 82

Figure 35. Example showing differences in nuclear spatial density due to change in cell size.

Figure 36. Schematic diagram explaining the derivation of (a) Normalized longitudinal migration index. (b) Normalized lateral displacement index

Figure 37. Comparison of nuclear pattern features of a muscle at three different time points .85

Figure 38. Segmentation evaluation. .88

Figure 39. Graph comparing F-measure at different stages of development for four muscle cells.

Figure 40. Performance evaluation of nuclear classification at different threshold values and different development stages.

Figure 41. Muscle development during the different stages of metamorphosis 103

Figure 42. Defective development in prepupal lethal mutants. 103 
Figure 43. Knockdown of Tor and Rheb causes decrease in the width of the persistent muscles.

Figure 44. Silencing of $T s c 1$ and $T s c 2$, regulator of Tor, causes hypertrophy of persistent muscles.

Figure 45. Atg5, Atg9, Atg 12 and Atg 18 promote atrophy and central clustering of nuclei in persistent muscles.

Figure 46.Comparison of median elongation (a) and elongation-S (c) in Atg9 and Atg 18 RNAi. 116

Figure 47. Average extent of muscle cells in $\operatorname{Atg} 9$ and Atg 18 mutants. 116

Figure 48. Silencing of SNF1A causes cell death of persistent muscles. 117

Figure 49. Silencing of $C P 1$ and $R M 62$ and over-expression of $T O R^{T E D}$ causes cell death of persistent muscles. 118

Figure 50.Grunge knockdown inhibits the histolysis of DEOMs in segment 1 and 2.

Figure 51. Silencing of $C p 1$ affect the distribution of nuclei along the medial axis in persistent muscles.

Figure 52. Longitudinal nuclear migration index help quantify the effect of $C p 1$ knockdown on anti-polar migration of nuclei in persistent muscles. 121

Figure 53. Nuclear spatial pattern features help quantify the effect of autophagy related genes knockdown on nuclear distribution in persistent muscles. 122

Figure 54. Spatial nuclear density index help quantify the distribution of nuclei with respect to the muscle mass change.

Figure 55. Comparison of change in longitudinal nuclear spread during polar and antipolar migration between different genotypes 124 


\section{List of Tables}

Table 1. Conservation of genes involved in insulin receptor signaling and autophagy.

Table 2. List of phenotypic tests for different developmental stages during Drosophila

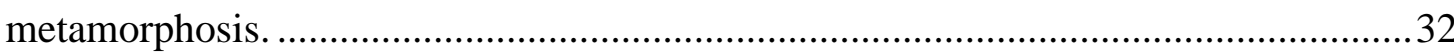

Table 3. Representation of nuclear tracks in tabular manner....................................... 78

Table 4. Comparison of the muscle segmentation performance of my method and a

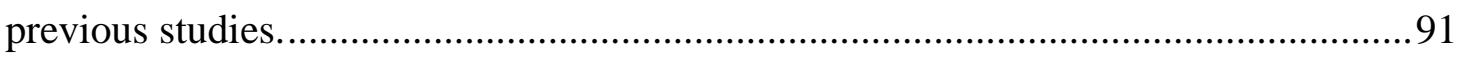

Table 5. Nuclear segmentation performance evaluation............................................93

Table 6. Comparison of performance of my nuclear classification with MTrack2 ......95

Table 7. Comparison between the findings of previous studies and results of our analysis for respective genes during Drosophila metamorphosis .............................................99

Table 8. Results of lethality test and muscle phenotype analysis............................. 102 Table 9. Time (Hours after head eversion) at which DIOM1 starts histolysing in different segments of the abdomen for Cpl and RM62 mutants. The yellow cell indicates the group of muscle cells in a sample which die at the same time in $C p 1$ mutant. ...........107 Table 10. Representative nuclear tracks. External nuclei corresponding to fragmented dead muscles move faster than nuclei tracked in remodelled muscles. 111

Table 11. Comparison of polar and anti-polar myonuclear migration between different genotypes 


\section{Author's Publications}

1. Kuleesha, Lin F, Wasser M: Spatial pattern analysis of nuclear migration in remodelled muscles during Drosophila metamorphosis. BMC Bioinformatics, 2017, 18:329, DOI 10.1186/s12859-017-1739-0.

2. Y. Kuleesha, W. C. Puah, and M. Wasser, "Live imaging of muscle histolysis in Drosophila metamorphosis," BMC Dev. Biol., vol. 16, no. 1, Dec. 2016.

3. Y. Kuleesha, W. C. Puah, and M. Wasser, "A model of muscle atrophy based on live microscopy of muscle remodelling in Drosophila metamorphosis," $R$. Soc. Open Sci., vol. 3, no. 2, p. 150517, Feb. 2016.

4. Kuleesha Y, Lin F, Wasser M: Muscle segmentation in time series images of Drosophila metamorphosis. IEEE Eng Med Biol Soc Conf 2015, pp. 30853088.

5. Kuleesha Y, Choo PW, Lin F, Wasser M: FMAj: a tool for high content analysis of muscle dynamics in Drosophila metamorphosis. BMC Bioinformatics 2014, 15(S 16):S6.

6. Kuleesha Y, Choo PW, Lin F, Wasser M: Study on muscle remodeling during Drosophila metamorphosis. Poster session presented at: Indian Ocean Rim Muscle Colloquium 2013; Singapore.

7. Kuleesha, Lin Feng, Martin Wasser, Quantitative time-series analysis of muscle cell dynamics in Drosophila metamorphosis, International Workshop on Advanced Image Technology (IWAIT'13), Nagoya, Japan, 7-9 January, 2013. 


\section{Abbreviations}

AHE: After Head Eversion

APF: After puparium formation

CLSM: Confocal laser scanning microscope

DEOM: Dorsal external oblique muscle

DFM: Direct flight muscle

DIOM: Dorsal internal oblique muscle

DLM: Dorsal longitudinal muscle

DVM: Dorsal ventral muscle

FMAj: Fly muscle analysis

GFP: Green Fluorescent Protein

IFM: Indirect flight muscle

LoG: Laplacian of Gaussian

MHC: Myosin heavy chain

MIP: Maximum intensity projection

mKO: Monomeric Kusabira Orange

MND: Myonuclear Domain

NSD: Nuclear spatial density

RNAi: RNA interference

ROI: Region of interest

TLM: Time-lapse microscopy

UAS: Upstream activation sequence 


\section{Chapter 1 Introduction}

Metamorphosis is a biological process that results in large scale morphological and functional changes in tissues. The transition from a larva to an adult fly involves extensive remodeling of cells and programmed cell death, which is controlled by a cascade of hormonal signals. Although metamorphosis is observed and studied primarily in insects and amphibians, understanding morphological changes in the muscular system may help reveal specific aspects of human muscle disorders. Our focus is on understanding the factors that contribute to the maintenance of the muscle mass by studying the genetics behind the processes that regulate post embryonic muscle development in Drosophila. Through genetic image-based screening we expect to reveal new gene functions and correlate them to understand the complex nature of the biological precursors to muscle remodeling.

\subsection{Skeletal Muscle Biology}

Skeletal muscle cells control most of the physical movements in our body, from simple actions such as smiling or frowning to critical movement of the diaphragm for breathing. Skeletal muscles also provide support to soft tissues, and regulate body temperature and carbohydrate and fat metabolism. Muscle movement is controlled by contraction and relaxation of skeletal muscle. This type of movement is achieved by the peculiar internal structure of muscles. Skeletal muscles are composed of several cylindrical muscle fibers. These fibers are striated, elongated, and multinucleate structures that consist of many myofibrils. The repetitive units of actin and myosin filaments form these myofibrils. The sliding motion of actin and myosin filaments causes the muscle to shorten (contract) or lengthen (relax), as shown in Figure 1 below: 

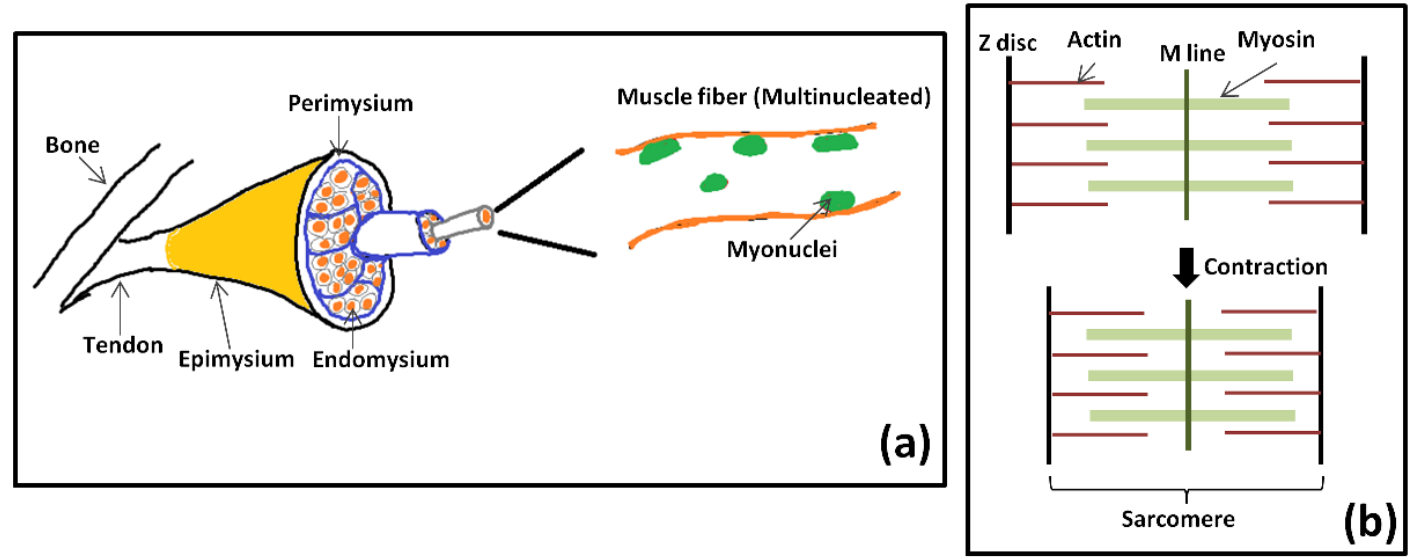

Figure 1.a) Anatomy of skeletal muscle b) Effect of muscle contraction in sarcomere.

\subsubsection{Significance of muscle mass change}

As illustrated in Figure 2, muscle mass and strength are essential for maintaining physical functions and mobility [1]. The association between change in muscle mass and muscle related disorders has been studied extensively in different organisms. In humans, loss of muscle mass or atrophy has been observed in cachexia (a wasting syndrome associated with chronic illnesses, such as cancer, AIDS, and sepsis), and age related weakening of muscles in sarcopenia, congestive heart failure, and muscular dystrophy. Disuse of muscles and starvation also leads to muscle atrophy. On the other hand, an increase in muscle mass, or hypertrophy, occurs with regular exercise, improved protein diet, and steroids. The growth of muscles induced by growth factors like IGF-1 and insulin receptors [2], [3] increases the cross-sectional area of muscles. However, in double muscling cattle [4], myostatin mutations lead to an increase in muscle mass, due to an increase in the number of muscles rather than an increase in their size (hyperplasia). Meat science is another area where improving skeletal muscle growth rate is used to produce high quality meat in livestock. An understanding of muscle growth mechanisms can also be used in clinical fields to prevent and cure muscle disorders. 


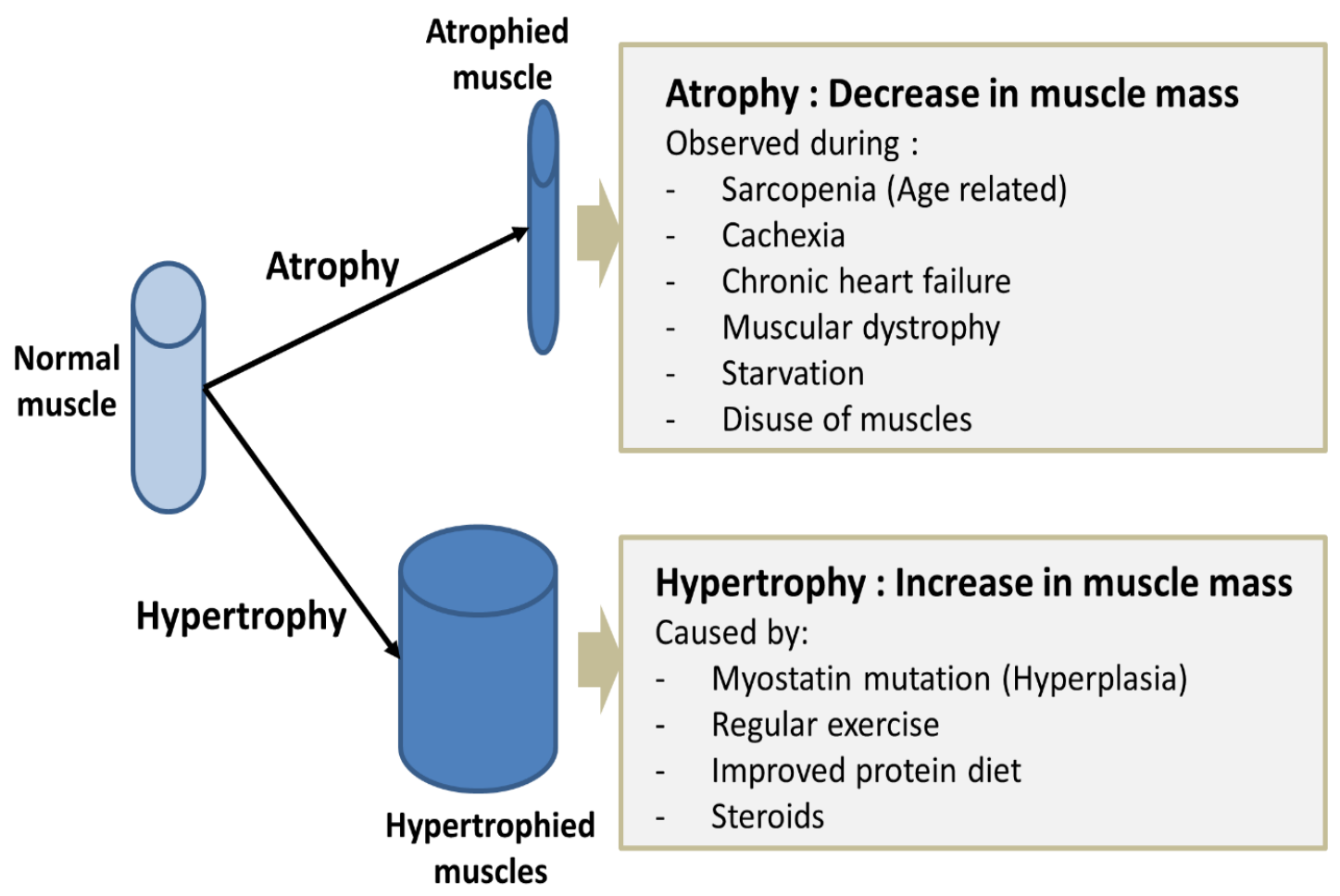

Figure 2.Significance of muscle mass.

\subsubsection{Molecular mechanism of muscle mass control}

Muscle mass is regulated by balancing two processes: protein degradation and protein synthesis. During atrophy the degradation of protein increases, while in the case of hypertrophy protein synthesis increases. Advances in the understanding of signaling pathways involved in muscle mass control would facilitate the identification of targets for pharmacological interventions for muscle disease. For example, in the case of atrophy treatment, the protein degradation pathway could be blocked or a higher rate of protein synthesis could be induced. The growth factors IGF-1 and insulin have been found to activate a downstream signaling mechanism that induces hypertrophy. The growth factor acts as an anabolic stimulus for the PI3-K/AKT pathway [3] and AKT reduces the expression of protein degrading ligands, thereby inhibiting protein degradation. Another downstream target of $A K T$ is $m T O R$, which is responsible for regulating protein synthesis.

$m T O R$ is also involved in another pathway that is associated with autophagy in skeletal muscles [1]. Figure 3 shows the association between the two pathways, 
PI3K/AKT signaling and autophagy machinery. Autophagy leads to the degradation of cytoplasmic components that provide amino acids during starvation [5]. It is also involved in degrading damaged organelles and toxic protein aggregates to maintain the health of muscles. The ubiquitin-proteasome and autophagy-lysosome systems are the major proteolytic pathways that control autophagy in muscles. During autophagy, the cytoplasmic components such as the mitochondria are enwrapped in a double layer membrane to form autophagosomes. The cytoplasmic content after fusion with a lysosome is sequestered by hydrolases that are present inside of it. In yeast, 16 genes (APG and AUT) [6] are associated with autophagosome formation. These proteins form complexes to regulate the induction of autophagy and autophagosome formation. As mentioned previously, $m T O R$ is also a critical regulator of autophagy induction. Activation of $m$ TOR by Insulin and IGF-1 through the PI3-k/AKT pathway suppresses autophagy [7], [8]. mTOR is also regulated by other factors, such as nutrients like amino acids, intracellular energy metabolism, and rapamycin. Many studies suggest that autophagic activity increases during muscle atrophy [9]. Age related muscle loss disorders including sarcopenia, and weakening and dilapidation of muscles have been associated with the activation of autophagy [10]. However, many aspects of the role of autophagy in muscle mass control are not yet understood. 


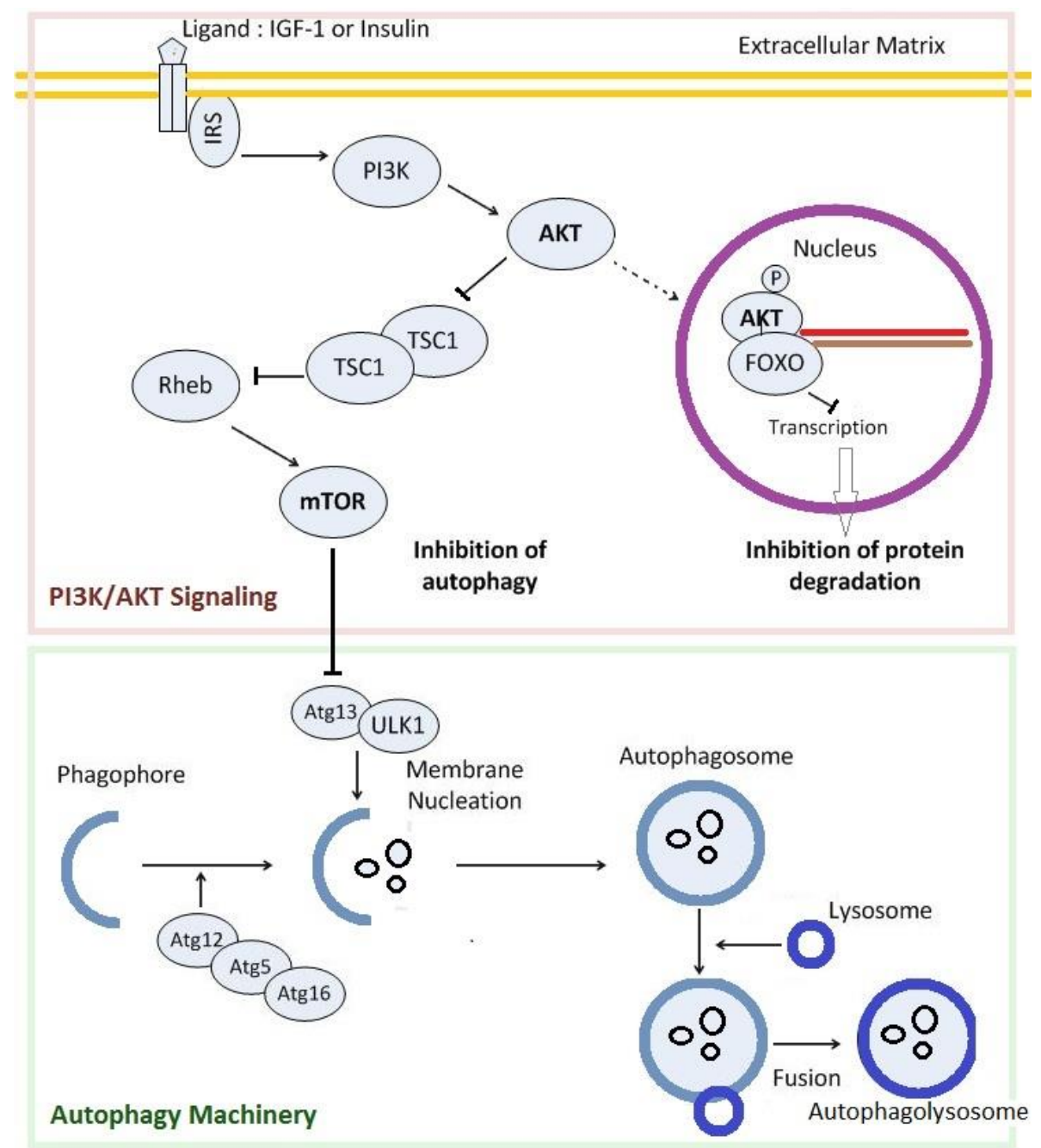

Figure 3. Regulation of protein metabolism by two pathways: PI3K/AKT signaling and autophagy mechanism.

\subsection{Drosophila as a Model to Study Muscle Mass Change}

\subsubsection{Muscle development in Drosophila}

Drosophila displays a holometabolous life cycle (i.e. before reaching adult form, they undergo an additional stage of metamorphosis, as shown in Figure 4). At the end of embryogenesis, the first instar larva hatches. It then goes through two more instar larval stages before turning into a pupa. Metamorphosis occurs during the pupal stage of the fly. At this stage, changes in the body structure of the larva take place through continuous growth and remodeling of the cells. After the completion of cellular restructuring the adults emerge from the puparium. The life cycle of Drosophila is 
approximately 9 days at a temperature of $25^{\circ} \mathrm{C}$, as illustrated in Figure 4. During development from embryo to adult, the muscles undergo immense changes in their pattern and morphology. Next, the details of muscle development in Drosophila are discussed.

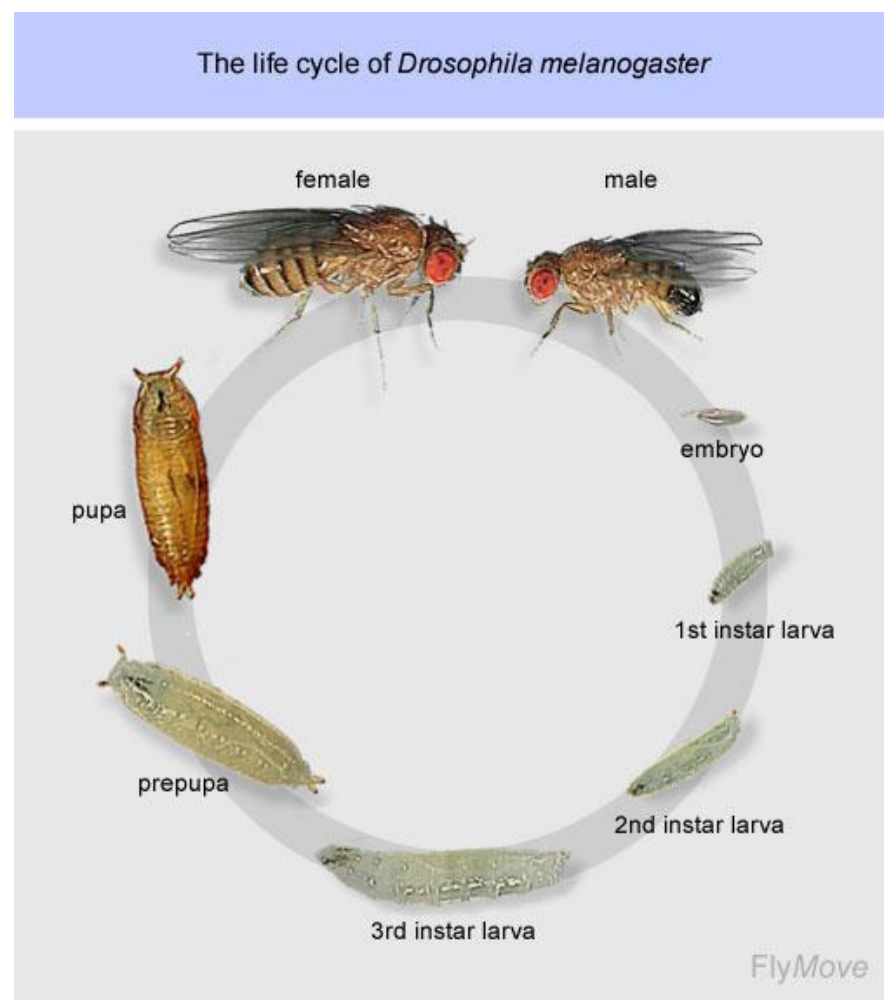

Figure 4. Drosophila life cycle [11].

\subsubsection{Embryonic and larval stage}

The mesoderm is one of the three primary germ layers between the ectoderm and endoderm that are formed during gastrulation. Apart from somatic muscle, other cell types such as gut lining, heart, and body fat also arise from the mesoderm. In an embryo mesoderm, cells start migrating dorsally and are partitioned into segments along the anterior posterior axis. Each segment gives rise to a population of different progenitors including ones for somatic muscles. Therefore, the myogenic lineage originates from segments in the mesoderm. The interplay of many genes controls these developmental processes. Many studied have been dedicated to understanding the pattern of expression that leads to the conversion of selected mesodermal cells to progenitor cells that are engrained with the information that is necessary for muscle formation [12]. The progenitor cells divide into two daughter cells; one of them initiates muscle formation 
in the embryo, the embryonic founder cell, while the other is a precursor to adult myoblasts. The embryonic founder cells fuse with the neighboring "naive" fusion competent myoblasts [13]. The characteristics of muscles, such as their size, location, site of attachment to the epidermis, and innervation, are decided by the founder cell. Since the fusion competent myoblasts cannot form muscle cells independently, their fate depends on the founder cells. After multiple processes of fusion with myoblasts, a syncytial muscle fiber is formed. The smallest muscle in an embryo is formed from 35 cells, while the largest is formed from 30-35 cells [14]. The adult precursor cells remain undifferentiated from an embryo until pupariation. However, during the larval stage they proliferate extensively. Figure 5 shows the muscle development during the embryonic, larval, and pupal stages in Drosophila.

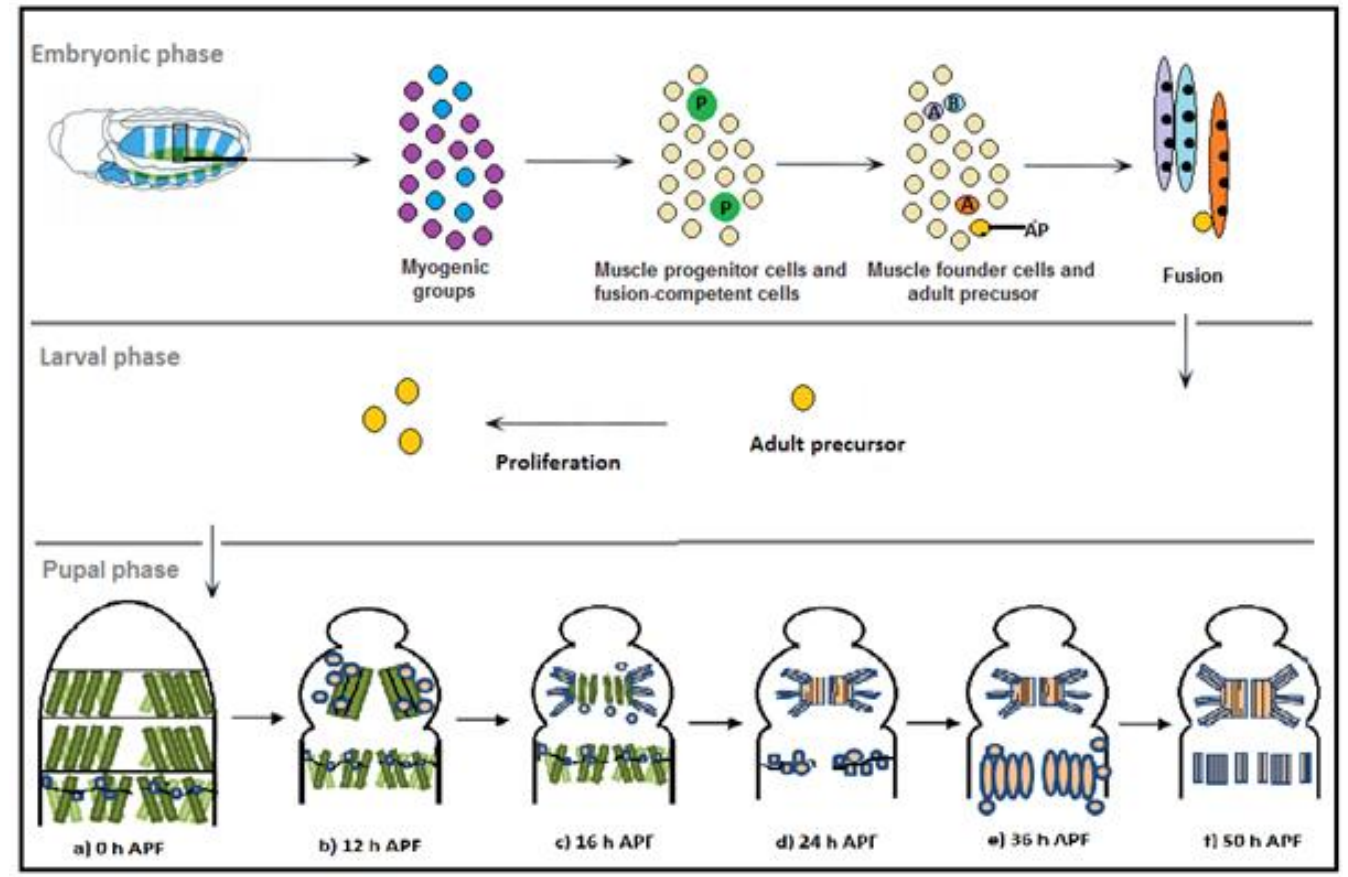

Figure 5. Muscle development in Drosophila at embryonic, larval and pupal stage.

\subsubsection{Metamorphosisłpupal stage}

During metamorphosis, large scale development and remodeling of tissues that led to the formation of adult body segments is observed. The head, thorax, abdomen, wings, and other structures start to take form, similar to adults. The muscles that are present in the larval stage are formed by the fusion of myoblasts in an embryo, as explained in the previous section. Most of these larval muscles undergo histolysis 
during metamorphosis [15]. The muscles that survive histolysis act as a template for fusion with the adult precursor cells to form adult muscle fibers [16], [17].

In Drosophila, metamorphosis is controlled by the changes in ecdysone titer [18], [19]. The first pulse of ecdysone in a larva triggers the formation of pre-pupa and the histolysis of thoracic larval muscles [14]. The pre-pupa develops over the next 10-12 hours, after which the ecdysone titers begin to rise again. This pulse triggers the prepupal-pupal transition marked by the eversion of the head. The thoracic muscles complete histolysis around 12 after puparium formation (APF). Only three thoracic oblique muscles survive (Figure 5a). These muscles start splitting by 16 APF and later fuse with myoblasts to form dorsal longitudinal muscle (DLMs) (Figure 5c). Dorsal ventral muscles (DVMs) are formed by de novo fusion of myoblasts. By 24 APF, each hemi-segment in the thorax has a DLM bundle with six fibers and three DVM bundles (Figure 5d). Muscle formation occurs later in the abdomen than in the thorax. Histolysis of abdomen larval muscles begins at around 20 hours APF (Figure 5d). The myoblast fusion in the abdomen begins at around 28-30 hours APF. By 50 hours the formation and patterning of the adult muscles is complete and each hemi-segment has a fixed number of dorsal, ventral, and lateral abdominal muscles (Figure 5f). The adult muscles are comprised of flight muscles (thoracic) and abdominal muscles. The direct flight muscles (DFM) control the orientation of the wings, while the indirect flight muscles (IFM) support the movement of wings. IFMs are large and are formed from DLMs and DVMs [14]. The muscles in the abdominal region control the movement and copulation in adult flies.

The adult muscle cells are multinucleated and are comprised of many fibers, while the embryonic muscle is a single multinucleated fiber. The structure of adult muscle cells in Drosophila is similar to the skeletal muscles in vertebrates. The process of development of muscles in vertebrates and Drosophila share many fundamental similarities. One of them is the formation of muscles through the fusion of myoblasts. Although muscle formation in vertebrates has only been studied in vitro, Drosophila provides an in vivo approach to understanding the mechanism behind muscle development. 


\subsubsection{Signalling pathways regulating metamorphosis}

$E c R$ or ecdysone receptor, one of the most extensively studied genes, plays a significant role in the steroid regulation of metamorphosis in Drosophila. The steroid hormone ecdysone binds to $E c R$, leading to the activation of a regulatory hierarchy of genes that are responsible for induction and differentiation processes during metamorphosis. The puparium formation is initiated by a release of ecdysone. The level of ecdysone begins to rise at 10 hours APF again and is at its peak at approximately 12 hours APF; this marks the transition from the pre-pupal stage to the pupal stage [19]. Many studies have shown that tissues such as the salivary gland [20], larval midgut, muscles, [21] and fat bodies [18], [22] undergo programmed cell death in a precise stage specific manner in response to ecdysone pulse. Apoptosis machinery also plays a role in programmed cell death during Drosophila metamorphosis [18].

The target of rapamycin (Tor) plays a critical role in the regulation of autophagy. In a study on fat body cells of Drosophila, autophagy was induced by the mutation of Tor or the repression of Tor through the PI3K pathway [22]. The PI3K pathway plays a crucial role as mediator of skeletal muscle hypertrophy [2], [23]. Autophagy inhibition has also been observed in the salivary gland through Tor signaling. Another study where autophagy was induced by rapamycin, an inhibitor of Tor, proved the role of Tor in autophagy regulation [24].

Tor acts as a mediator between the PI3K pathway and autophagy machinery. It represses autophagy through the phosphorylation of Atg 1 , one of the autophagy related genes. Autophagy machinery is comprised of many Atg genes that were initially found in yeast; however, not all of them are conserved in Drosophila. In Drosophila, the Atg1-Atg13 complex induces the formation of autophagosomes. Autophagosome nucleation requires the accumulation of phosphatidylinositol-3-phosphate (PI3P) enriched structures, which bind with other Atg proteins like Atg18, Atg6, and Atg14 to assist membrane trafficking. Vesicle expansion is regulated by two ubiquitin-like complexes, Atg5-Atg12-Atg6 and Atg7-Atg8. Drosophila has two Atg8 genes (Atg8a and Atg8b), and both localize to autophagosomes.

\subsubsection{Advantages of Drosophila for genetic studies}

Although more than 20,000 genes have been identified in humans, the functions of 
a large number of genes remains unknown even today. In order to understand the genetics of human diseases, the underlying genes and their functions must be identified. One of the most common methods for studying functional genomics is the use of mutant lines from different organisms to create a model of human diseases, such as neurological disorders and cancer. However, this methodology depends on the level of the evolutionary conservation of genes in an organism. A database called Orthodisease [25] lists the orthologs for human disease genes in various model organisms. The database identified 2269 orthologue clusters in Musmusculus, 1254 in Drosophila melanogaster, 1186 in C. elegans, and 202 in E. coli. This accounts for approximately $75 \%$ of human disease genes that have conserved homologues in $D$. melanogaster. Table 1 [26]-[28] shows some of the conserved genes of pathways that are involved in muscle mass regulation (e.g. the insulin receptor signaling pathway and autophagy machinery). The higher number of conserved genes is not the only reason that Drosophila has been favored by researchers as model organism. Drosophila, "the golden bug", [29] has several other features that make it ideal for studying genetic perturbation, especially in high throughput studies. It has a shorter lifespan (11-14 days), is smaller in size, and is cheaper to maintain in large numbers. Drosophila lines and transgenic strain libraries are available from various stock centers. 


\begin{tabular}{|c|c|c|}
\hline $\begin{array}{l}\text { D. melanogaster } \\
\text { gene }\end{array}$ & Homo sapiens gene & Function \\
\hline DInr & insulin/IGF1 receptor & \multirow{5}{*}{ Insulin receptor pathway } \\
\hline Dp110 & $\mathrm{PI}(3) \mathrm{K}$ & \\
\hline DAkt1/dPKB & Akt/PKB & \\
\hline dS6K & S6K & \\
\hline Chico & IRS & \\
\hline dTOR & mTOR & Regulates protein synthesis \\
\hline $\operatorname{Atg} 1$ & ULK1, ULK2 & \multirow{2}{*}{$\begin{array}{l}\text { Autophagosome formation : } \\
\text { Induction }\end{array}$} \\
\hline $\operatorname{Atg} 13$ & HARBI1 & \\
\hline Atg6 & BECN1 & \multirow{2}{*}{$\begin{array}{l}\text { Autophagosome formation : } \\
\text { Membrane Nucleation }\end{array}$} \\
\hline CG11877 & ATG14 & \\
\hline $\operatorname{Atg} 3$ & ATG3 & \multirow{6}{*}{$\begin{array}{c}\text { Autophagosome formation : } \\
\text { Expansion }\end{array}$} \\
\hline $\operatorname{Atg} 4$ & ATG4A,B,C,D & \\
\hline $\operatorname{Atg} 5$ & ATG5 & \\
\hline $\operatorname{Atg} 7$ & ATG7 & \\
\hline Atg8a, Atg8b & $\begin{array}{l}\text { LC3, GABARAP, } \\
\text { GABARAPL2 }\end{array}$ & \\
\hline $\operatorname{Atg} 12$ & ATG12 & \\
\hline
\end{tabular}

Table 1. Conservation of genes involved in insulin receptor signaling and autophagy.

\subsection{Nuclear Positioning in Muscles}

Skeletal muscle fibers are one of the largest cells, and they are multinucleated. It has been thought that the nuclei inside of muscle cells are positioned such that the nucleus to cytoplasm ratio would remain fixed [30]. One of the reasons for this possible behavior is that a nucleus can only support a fixed volume of cytoplasm, called a myonuclear domain (MND) [31], due to the limited distance that the protein can be transported (Diffusion model) [32]. Therefore, it is expected that in a healthy muscle, the nuclei would have maximum internuclear distances. A quantitative study on the spatial distribution of nuclei in mice confirmed that the nuclei are not randomly distributed inside of muscle and are arranged in a row-like formation, indicating that the nuclei could be repelling each other to minimize the transport distance [33]. Unlike 
healthy muscles, several studies have revealed that the MND size varies in hypertrophic and atrophied muscles [34], [35]. Centrally positioned nuclei have been observed in many muscle disorders, including central nuclear myopathies [36] and muscular dystrophy [37, p. 1], [38]. Previous studies have shown that KASH and SUN proteins play a role in regulating nuclei positioning [39], [40]. In a study on Drosophila larvae, the KASH (klar and msp-300) mutants showed impaired locomotion and aggregation of the myonuclei [41]. In another study, the loss of JNK signaling also resulted in the clustering of nuclei and large spaces in muscle cells devoid of nuclei [42]. Despite the studies conducted on nuclear positioning, its role in muscle function is not clear.

\subsection{Imaging as a Tool for Genetic Analysis}

\subsubsection{Light microscopy}

Light microscopy enables the observation of microscopic objects by illuminating them with a light source. It has been one of the most widely used research tools for centuries. From the first published work on a two lens compound microscope by Robert Hooke in 1665 , to the current automated microscopes with multi-focal and live cell imaging capabilities, microscopy has observed immense innovation and evolution to cope with the increasing demands of biological research [43].

There are many light illuminated microscopes for two dimensional imaging, such as bright field, dark field, and phase contrast [44]-[46]. These microscopes use a common configuration of focusing the light from a condenser lens onto a specimen and then collecting the transmitted or scattered light with an objective lens. Another microscope that is commonly used to visualize biological samples is a fluorescence microscope [47]. The florescence microscope uses light of a specific wavelength to illuminate the specimen and collect reflected light rather than transmitting it. It exploits the tendency of florescent probes to emit light of a given wavelength when excited with a laser, to view specimens that do not fluoresce on their own. Light sources like mercury lamps produce light, from which a specific wavelength called an excitation wavelength is extracted by an excitation filter. This light is focused on the specimen through the objective lens. After absorption of light, within nanoseconds, the fluorophore emits light that is filtered by the emission filter and captured by the detector. The difference 
between excitation and emission wavelengths is called Stokes shift; this is a significant property for determining the quality of a fluorophore. A higher Stokes shift makes it easier to distinguish an emission spectrum from an excitation spectrum. Other important properties of a fluorophore include lifetime, extinction coefficient, quantum yield, and photo stability.

Various types of fluorophores are available for staining biological samples. Fluorescent stains work by attaching to specific biological molecules. For example, DAPI is used to bind to nucleic acid groups for labeling cell nuclei; fluorescently labeled phalloidin is used to stain actin fibers in mammals; and Alexa Fluor Dye, highly resistant to photo-bleaching, indirectly binds to a molecule of interest. Quantum dots [48] are another potential non-organic fluorophore for fixed and live specimens. They are made of semiconductors and are largely used in confocal microscopy. However, the discovery of fluorescent proteins opened a new aspect of fluorescent imaging. Fluorescent proteins are produced within living cells and they undergo biological processes similar to the other proteins. These proteins are bright and non-toxic; thus, they can be used to monitor cell and tissue development. The first fluorescent protein that was discovered is the green fluorescent protein (GFP), which was extracted from Aequorea victoria, the Pacific Northwest jellyfish.

The concept of florescence microscopy and the availability of fluorophore has paved the way for the development of many advanced florescence microscopes, including the confocal laser scanning microscope (CLSM) [49] and light sheet fluorescence microscopy [50]. CLSM works on the same principal as fluorescence microscopy, but it overcomes the problem of out-of-focus blur, which most of the aforementioned microscopes suffered from. Out of focus rays come from the illumination of the sample at planes adjacent to the plane of focus, especially in thick specimens. CLSM overcomes this problem by filtering the out of focus rays by using a pinhole detector conjugate on the sample plane. Another advantage of a confocal microscope is the collection of z-slices at multiple planes of the sample, thus making it a useful tool for 3D visualization and analysis. However, performance with this microscope is reduced considerably if the thickness of a sample is more than 200 microns.

An advanced form of a confocal microscope, called two-photon excitation microscopy [51], is used for thicker tissues of $1 \mathrm{~mm}$. It uses two low energy photons to 
excite the fluorophore, compared to one high energy photon. It comes with three advantages: First, it uses longer wavelengths of infrared light to minimize scattering. Second, the absorption of two photons suppresses background signals. Finally, low energy excitation causes less bleaching. A multi photon excitation microscope uses multiple photons to excite the sample rather than two-photons. A spinning disk microscope [49] is also a confocal microscope that is designed to conduct fast scanning. The original confocal microscope can perform only point scanning; thus, it is slow. The spinning disk microscope has a Nipkow disk with multiple pinholes that are located on a conjugate image plane. The excitation laser is passed through the pinholes in a rotating disk to raster scan the specimen. The emitted light is then collected through the same pinhole or the opposite one. The right selection of size and distance between pinholes is important for avoiding pinhole crosstalk.

\subsubsection{Live cell imaging}

Before the 1990s, most of the work in fluorescent imaging was done on fixed specimens. Although the GFP was discovered by Shimamuro in 1962, it was not until the late 1990s that GFP became popular as a labeling technique for live cells. The ability of fluorescent markers to visualize a specific biological process without affecting the global physiology of the cell has made it an ideal tool for studying gene functions. Since the discovery of GFP, several other sophisticated fluorescent proteins have been identified, such as RFP, YFP, enhanced-GFP, and split GFP. These fluorescent proteins can be used to study various biological processes, such as protein interaction, direct measurement of cell parameters and state, organelle function, and cell motility. Recently, a genome wide phenotypic profiling was achieved in human Hela cells using time lapse imaging [52]. The purpose of this was to interpret the functions of mitotic genes like cell division, migration, and survival. The advantage of time lapse imaging is the ability to detect the transient behavior of biological entities, given that an appropriate interval for imaging is used. Research on muscles cells is also highly dependent on live cell imaging, since they are one of the few cells that can be cultured in media. For example, in a study of genes involved in muscle assembly, a primary muscle cell culture derived from Drosophila embryo was analyzed using fluorescence microscopy [53]. 
Another major advancement in this field is in vivo live cell imaging. This has been a breakthrough for research in developmental biology. With live cell imaging on cultures, it is possible to understand the changes in the physiology of cells, but it is not possible to provide $3 \mathrm{D}$ and temporal information about the changes in their natural environment. In vivo imaging is used to study disease models by monitoring gene expression in a targeted tissue in an organism. Thus, large scale analysis of biological processes would require imaging of the whole organ or organism. During the last decade, this technique has largely been exploited to visualize organisms such as $C$. elegans, Drosophila, and zebrafish. However, this technology is in its nascent stages and is crippled by complications and restrictions. Currently, time lapse in vivo imaging is possible only in small translucent organisms. Even the latest live imaging confocal microscope can only image up to 0.22 micrometers of depth in tissue, which further limits the options for studies. There are several optical limitations, but the pace at which technological advances are occurring, the development of versatile and multifunctional microscopes could circumvent these limitations in the near future. Another problem is the robustness of the labeling markers when using automated fluorescence imaging (i.e. time lapse imaging). For high-throughput phenotypic profiling experiments, the labeling and detection procedure should be highly reproducible and quantitatively assessable at different time intervals.

Despite these disadvantages, time lapse in vivo imaging has established a new trend in functional genomics experiments. When applied to genome wide screening techniques like RNAi, it becomes a powerful technique for high throughput automated and quantitative analysis of biological responses in living cells of whole organisms. In Drosophila, in vivo imaging has been used to observe embryogenesis and metamorphosis. The biological advantage of using a semitransparent organism at both stages has made it an ideal system for studying pre-natal development. The developmental responses of ecdysone, a critical regulator of metamorphosis in Drosophila, was studied by in vivo imaging of a larval salivary gland, imaginal discs, and abdominal muscles in Drosophila prepupa [54]. The UAS-GAL4 system was used to express GFP in respective tissues. Time lapse images were acquired using a modified Zeiss Axiophot microscope. Another In vivo imaging of Drosophila pupae was conducted using mesoscopic fluorescence tomography [55]. Fluorescence tomography has been reported to have greater penetration depths than fluorescent microscopy. 


\subsection{Image-based Phenotypic Analysis}

Fluorescence imaging based screen has been used extensively for phenotypic analysis in various cell types, including Hela, C2C12 cultures, and in in vivo organisms like Drosophila, C. elegans. With the availability of automated microscopy facilities, fluorescence imaging has the potential to be used for medium-large scale genetic profiling experiments. During the last decade, large scale microscopy screening has been used in various studies to identify genes that regulate specific biological functions. In most of these experiments, fluorescence images were manually categorized into different phenotypic classes [56], [57]. Although they were able to analyze gene functions at a system level, the manual annotation was time consuming and may have introduced human errors. The enormous data size, resulting from a large number of genetic screens with the possibility of multichannel, 3D, or time lapse imaging would make it impossible to conduct a manual investigation. A standard and unbiased numerical profiling of the screens requires an integrated workflow of automated image processing software. In general, the image processing pipeline that is used by most imaging based screens is described in the following section.

\subsubsection{Image processing}

Most images face the problems of non-uniform illumination, blurring due to optical noise, and debris or uninteresting objects. Median filters, convolution operations, and histogram-based contrast enhancement are various techniques for removing noise and improving the contrast of the object of interest. In addition to these common problems, fluorescence imaging in live samples often suffers from artifacts, such as attenuation of excitation and emitted light, and misalignment of adjacent z-slices. The intensity attenuation is corrected by approximating the intensity of each slice with a constant attenuation coefficient. However, the model only works with samples of the same type of tissues; thus, the same local attenuation properties. A commonly observed problem in $3 \mathrm{D}$ imaging is that the objects in adjacent z-slices do not align because of the movement of the sample during imaging. Registration techniques are used to align zslices and obtain a corrected 3D view of object of interest. Deconvolution is an advanced technique that is used to reduce out of focus fluorescence in 3D image stacks. 
Image processing often requires data reorganization that caters to software requirements. Conversion of images from one type to another (e.g. .LSM to .TIFF), stitching of images when a whole specimen cannot be imaged at a specific magnification, and maximum intensity projection of 3D slices for the study of a large number of time lapse images are some examples of image manipulation techniques used in biological image processing.

\subsubsection{Image segmentation}

The next step is to identify the object of interest in the image and extract its boundary. Segmentation techniques can be broadly divided into the following categories:

\subsubsection{Thresholding}

This technique is useful when there is a distinct segregation between the object of interest and the background. For such cases, automated thresholding methods like Otsu, Isodata, and max entropy are available; however, these methods do not provide good results if images suffer from non-uniform intensities and background noise. This problem is solved by local adaptive thresholding (i.e. calculating different thresholds for different regions). Computational analysis of mitotic phenotypes often applies local adaptive thresholding to segment nuclei [58], [59].

\subsubsection{Region based segmentation}

Region growing methods require the selection of seed points. On the basis of the homogeneity criterion, the neighboring pixels of the seed are either combined with the seed to form a region or left for evaluation by other seeds. Seeds can be selected manually or in an automated manner using low level filters like the Harris corner detector. Another option for region growing is to select regions instead of seeds and grow each region by adding neighboring regions [60]. This technique works on the homogeneity of pixels within a region and is suitable for large-scale image segmentation.

Watershed [61] is another region based segmentation method in which the image is divided into topographical regions, such that the region represents the catchment basin 
and the boundaries of the basin are high gradient edges. However, it has many disadvantages such as over segmentation and sensitivity to noise. This technique has been used with other powerful segmentation techniques to overcome its drawbacks. In nuclei segmentations, watershed split-n-merge is used to correct the erroneous over segmentation [59]. In some methodologies, watershed is used as an over segmentation technique that is followed by region merging [62].

\subsubsection{Edge-based segmentation}

Edge-based segmentation is one of the conventional methods for finding edges in an image. A Sobel edge detector [63] calculates the first order derivative using a threeby-three convolution kernel, while a Laplacian computes the second order derivative of an image. In addition, a canny edge detector [64] produces continuous edges by using double thresholding. Edge-based segmentation techniques are computationally less expensive. However, in cases with irrelevant objects and irregular gradient they fail to produce correct boundaries.

\subsubsection{Deformable Models}

Deformation based models like active contours [65] or level sets [66] use mathematical models to represent shapes with geometry and elastic curves. They work on the principle of minimizing the energy associated with a contour to fit the boundary of an object. Because of the topological flexibility and accuracy, they are widely used in medical image segmentation. However, they are sensitive to noise and because of manual initialization, they are not suited for large scale image segmentation.

\subsubsection{Graph-based Methods}

The graph based methods work on a global optimization criterion to segment images. The image is represented as a graph, where each node corresponds to a pixel or region and each edge corresponds to the weighted affinity between nodes. The graph is partitioned into subgraphs by minimizing certain cost functions; various cost functions have been proposed to solve graph partitioning problems. Minimum cut [67] minimizes the aggregate cut value between subgroups, while normalized cut [68] considers the connectivity of one node to all other nodes in the graph. Graph based algorithms are computationally expensive and sometimes lead to over segmentation. 


\subsubsection{Cell tracking}

In time series analysis, tracking cells throughout frames is important to understand their transient behavior. The tracking algorithm can be applied directly to the segmented cells. The correspondence between the same cells in adjacent frames can be created on the basis of the distance between centroids, rate of motion, or shape of the cells. It is important to consider cases where two cells merge or split, or they go outside of the window of the image [59].

\subsubsection{Classification}

The most commonly used methodology for the classification of biological entities is to extract features from each segmented object and classify them on the basis of the phenotypes observed. A segmented biological object can be quantitatively described using various types of features including basic features like area, perimeter, centroid, orientation, and average gray level; shape features such as circularity, convex hull, and granulometries; texture features including Haralick features (entropy, homogeneity), gabor filters, and LBP; and advanced features such as scale invariant feature transform (SIFT), speeded up robust features (SURF), and histogram of gradient (HOG).

After selecting appropriate features, the cells are classified into user defined classes (e.g. mitotic phenotype: interphase, prophase, and telophase). Several supervised classifiers like neural network and support vector machine (SVM) are available and can be trained to discriminate between the phenotypic classes. The accuracy of the classifier relies on the user labeled ground truth. In addition to being time consuming, it is possible to have inconsistencies due to labeling by different users. Although supervised classification is still a favored method, many studies have used unsupervised techniques for phenotypic classification. One such study on Hela cells used the Gaussian mixture model with temporally constrained combinatorial clustering for the prediction of cell morphology (interphase, prophase, anaphase, etc.)[69]. Incorporating temporal information into a classifier model using the hidden Markov model (HMM) has improved classification accuracy [59], [69]. The transition probabilities of phenotypes were used to predict cell behavior. 


\subsubsection{Data mining}

The quantitative results of all of the screens can be compared using data mining tools. A control experiment that was conducted under the same conditions as other specimens is used to compare differences in phenotypes. The "phenoprint" [70] or quantitative signature of each dataset or biological entity is defined by specific parameters. These parameters can be clustered to identify groups of datasets that have similarities. For example, in experiments where mutants are created by genetic perturbation, the phenoprint of the datasets can be clustered to predict a group of genes that are involved in the same biological function. Usually, the comparison of parameters between datasets is a two dimensional problem; however, in the case of a time series analysis, the complexity increases. Event order maps [71] are used to represent a dataset in the form of successive phenotypic events. The phenotypic events are aligned temporally around a reference phenotype. The difference between sequences of events is used to measure the dissimilarity between datasets. Another method is to discretize the pattern of changes in parameter, using piecewise aggregate approximation (PAA).The PAA coefficients are represented symbolically, based on their locations between breakpoints [72]. There are several other methods that are used to analyze time series data; the appropriate method for an experiment largely depends on the type of parameters that are extracted and the expected outcome of the analysis.

\subsection{Related Work on Postembryonic Muscle Development}

The role of various genes in post embryonic muscle development has been studied in vivo and in cultures of Drosophila to understand diverse processes such as muscle remodeling, morphogenesis, and programmed cell death. During the last decade, few studies have used in vivo imaging to visualize the histolysis of larval muscles and the formation of adult muscles. Dutta et al. [73] used Gal4-UAS driven expression to image the histolysis of both thoracic and abdomen muscles and the remodeling of surviving internal dorsal oblique muscles into the dorsal longitudinal muscles (DLMs) in pupa. A similar study on abdominal muscle visualized the dynamics of muscle remodeling during the pupal stage and reported that the abdominal muscles fail to die in $r b p$ mutants of $B R-C$ [54]. Time lapse in vivo imaging was also used to study the role of EAST and 
Chromator in the remodeling of muscle cells during Drosophila metamorphosis [74]. This study proved that Chro and east act in an antagonistic manner. East inhibits the histolysis of larval muscle cells while Chro promotes it.

Another study showed that the myofibril structure of indirect flight muscles (IFMs) is interchangeable between fibrillar and tubular types, depending on spalt switch during metamorphosis [75]. Stretch activated fibrillar myofibres are found in flight muscles, while slow moving muscles like those in legs have a tubular structure. In this study, RNAi screening was used to identify the function of the transcription factor spalt major (salm). In a recent study by Schnorrer et al., genome scale analysis was done on Drosophila muscles to identify morphological phenotypes observed by disrupting genes [76]. In this study, the muscle specific mef2-GAL4 line was crossed with 17,759 UAS-Gene RNAi lines. All of the genes that scored defective in muscle screens were classified on the basis of muscle, myofibril, and sarcomeric morphologies. This comprehensive analysis divided a cohort of genes into welldefined morphological phenotypes that were observed at different stages of development in Drosophila. However, only a limited number of phenotypic classes were analyzed in this study and the progression of muscle defects was not targeted. In a recent study on programmed cell death, it was found that apoptosis, instead of autophagy, plays a role in histolysis of the abdominal dorsal external oblique muscles (DEOM) during Drosophila metamorphosis [21].

Although many studies have used in vivo imaging to analyze muscle cell dynamics, few have performed a quantitative analysis. A study by Chinta et al. [77], characterized the phenotypic changes in muscles induced by RNAi through the quantitative analysis of time lapse in vivo images of Drosophila pupae. A confocal laser scanning microscope was used to collect images at multiple planes (3D) of the pupae. By segmenting the muscle cells using a semi-automated level set based technique, they were able to visualize the muscle cells. The patterns in muscle morphological changes in mutant EAST(1-100902)-GFP and control genotypes were measured by extracting shape features, such as volume, muscle width, and extent over multiple time points. 


\subsection{Aim of this Research}

The comprehensive study done by Schnorrer et al. provides a platform to extend research into discovering new gene functions and networks associated with muscle development [76]. Since muscle remodeling during metamorphosis is a highly dynamic process that is controlled by programmed activation of genes and secretion of hormones, it would be more interesting to observe the progression of changes in muscles throughout the developmental period. As, discussed in previous section, study on muscle remodeling throughout Drosophila metamorphosis on large number of genes has not been attempted before. With the availability of a genome-wide RNAi library for Drosophila and in vivo imaging tools, muscle development in Drosophila during metamorphosis can be used as a model to study genes that regulate skeletal muscle mass.

The goal of this study is to design a framework of genetic screens and time lapse image analysis techniques to study large number of genes involved in muscle remodeling during metamorphosis by characterizing the phenotypic changes in muscle cells that are caused by genetic perturbations. This objective has been divided into two parts. First, using time lapse in vivo microscopy for qualitative image analysis of muscle specific phenotypic changes caused by genetic perturbation. The purpose of this step is to shortlist genes which showed new transient muscle phenotype which have not been observed in traditional end-point assays in previous studies. Second, quantitative analysis to statistically confirm the findings in previous steps as well as identifying phenotypes which could not be distinguished visually. In order to achieve this objective, my solution was to design a custom tool for muscle image analysis which could manage large number of muscle images and their metadata, perform quantitative analysis of muscle morphological changes and myonuclei spatial patterns with least manual intervention and provides interactive interfaces for exploring muscle phenotypes. Figure 6 shows the workflow of the muscle analysis framework which includes the biological experiments and computational image processing. The contributions of the thesis have also been listed in the diagram. 


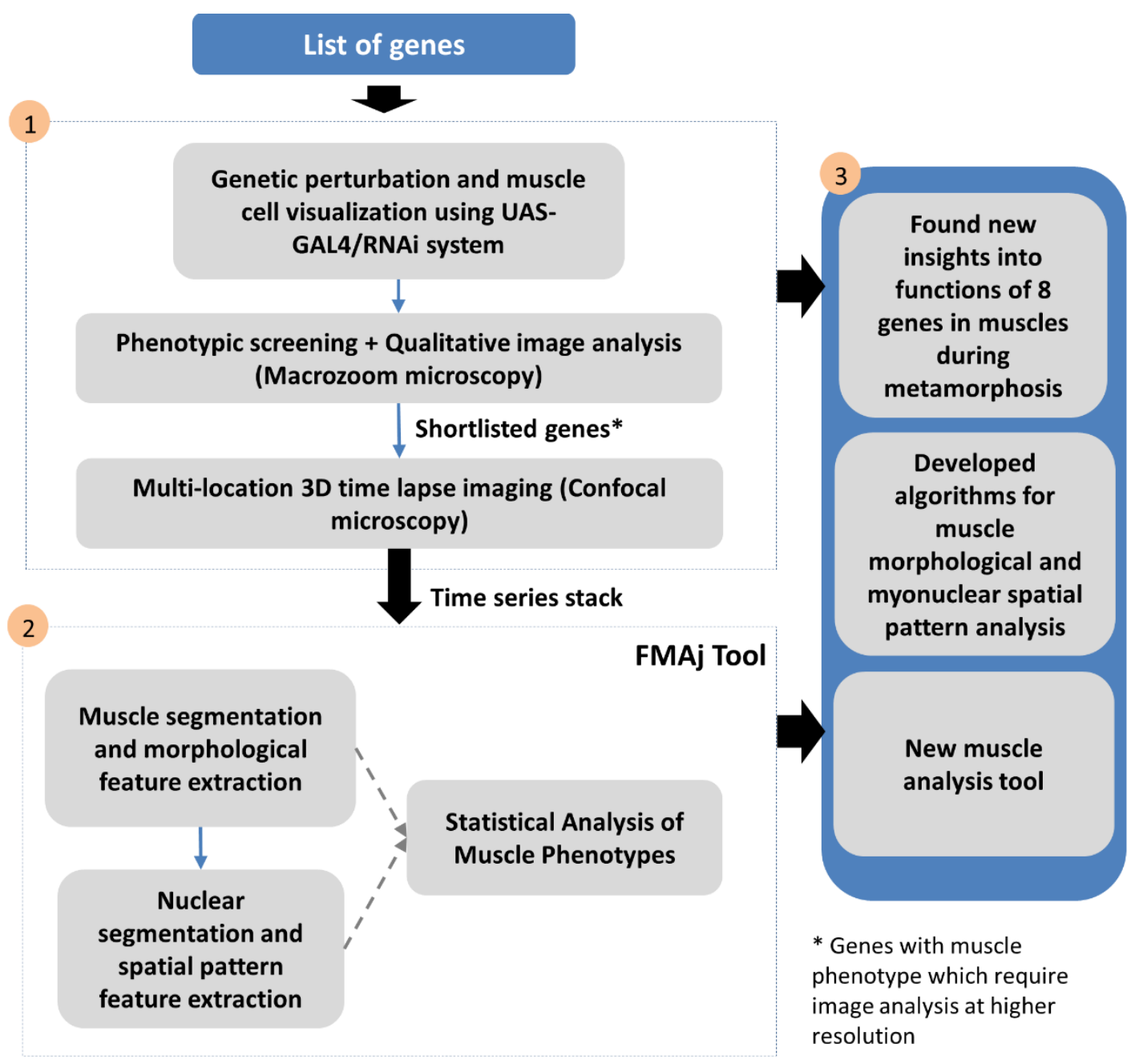

Figure 6. Workflow of the muscle analysis framework and contributions of this thesis.

\subsection{Organization of the Thesis}

First, the transient muscle phenotypes caused by genetic perturbation were captured through time lapse imaging of live pupae. The details of genetic screens and image acquisition protocols are discussed in Chapter 2.

Next, the phenotypic changes in muscles and myonuclei that are caused by genetic perturbations are characterized. A detailed phenotype analysis would require an assay of a large number of mutants. Analyzing datasets of hundreds of mutants, each with multiple time frames, would have become a bottleneck in this system. Therefore, I designed algorithms that can characterize the phenotypes quantitatively in a semiautomated or automated manner. In Chapter 3, I'll discuss the design of muscle 
analysis tool (FMAj) in which the algorithm for quantitative phenotypic analysis of muscles are implemented.

Chapter 4 focuses on quantifying the morphological changes in muscle cells using a watershed based time series segmentation technique.

In Chapter 5, I'll discuss the nuclear segmentation and classification algorithms. The nuclear pattern features designed for analysis of myonuclear distribution are also described in this chapter.

The results of the analyses from Chapters 2-5 are discussed in Chapter 6. It includes the performance evaluation of algorithms and results of phenotypic analyses of muscles.

In Chapter 7, I'll summarize the major contributions of this thesis and discuss the future work.

The genetic screening protocol design and phenotypic analysis of images was done by me and Martin Wasser. Phua Wee Choo and I did the microscopy of Drosophila pupa. The computational pipeline and FMAj tool was designed by me under the supervision of both my supervisors. 


\section{Chapter 2 Experimental Work on}

\section{Genetic Perturbation and Imaging}

\section{Protocol}

We require an experimental system that can capture the transient phenotypes of muscles in vivo to understand the role of genes in muscle remodeling. Most of the research on muscles is conducted on cultured cells. Few studies have performed in vivo live cell imaging of muscles in Drosophila [54], [74], [76]. However, none of them have studied muscle remodeling throughout metamorphosis for large number of genes. In this study, we modified the genetic screening protocol used in Chinta et al. Mainly there are two differences. First, we used RNA interference (RNAi) in combination with a UAS-GAL4 system to target genetic perturbations in muscles. By using RNAi transgene available in stock centers for genetic perturbation instead of creating new transgene, we were able to assay large number of genes in less period of time. Second, in order to assay large number of genes, we designed a two level-phenotypic screening protocol. The purpose of level-1 screen is to select genetic perturbations which affect the muscle morphology or myonuclear distribution during metamorphosis, by time lapse in vivo imaging of muscles at large time intervals using a macro zoom fluorescence microscope. At level-2 screen, the selected genetic perturbations are used for imaging of muscles at higher resolution using confocal microscope. The first screen reduces the amount of resources which would have been required, if we did confocal imaging of every genetic perturbation. In this chapter, the techniques and protocols used for targeted genetic perturbation analysis and time lapse in vivo imaging are discussed. In the first and second section, we will discuss the details of targeted genetic perturbation using UAS-GAL4 system and RNAi. In the third section, the two levelphenotypic screening protocol is discussed. 


\subsection{Targeted Misexpression using the GAL4/UAS System}

The GAL4 system is a bipartite system, as shown in Figure 7; the driver transgene controls expression of GAL4 through a cell/tissue specific transcription factor while the responder transgene is activated by GAL4, which binds to the UAS and results in transcription of the target sequence [78, p. 4]. This design has two main advantages: First, the expression of a target gene can be controlled spatially and temporally by the appropriate choice of transcription factor binding sites for GAL4 drivers. For example, GAL4 can be selectively expressed in tissues like muscles or neurons. Second, it provides a controlled system (i.e. the presence of GAL4 would have no effect on cells if the fly did not have a UAS region, as a UAS will also not be activated without GAL4) and these flies will behave normally. Thus, both transgenes are necessary for this system to work.

In order to visualize a specific tissue, the UAS region is often associated with a target gene that expresses a fluorescent protein, such as a green fluorescent protein (GFP). GFP emits green light when exposed to blue laser and is used to visualize biological structures.

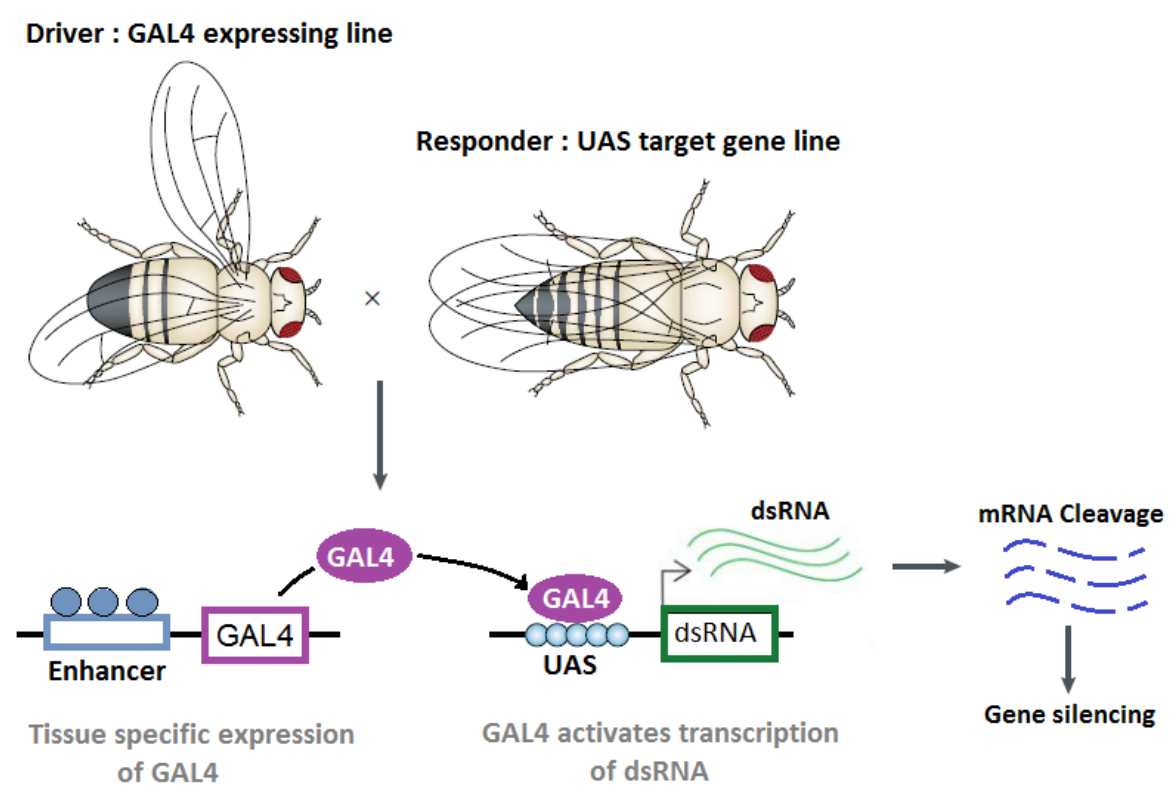

Figure 7. Tissue specific gene silencing by combining UAS-GAL4 system and RNAi approach.

First, a GAL4 line that expresses GAL4 in the desired tissue is selected and crossed with a reporter line that expresses fluorescent protein. In the offspring, the desired 
subset of cells will make GAL4, which binds to the UAS region and enables the production of fluorescent protein. Thus, the desired subset of cells will fluoresce and can be observed with a microscope. This technique can be combined with an RNAi approach to visualize the phenotypic changes caused by genetic perturbations in a specific tissue [79, p. 4].

\subsubsection{RNA Interference}

The RNA interference (RNAi), discovered by Andrew Fire and Craig Mello in 1998 [80], is a fast technique in which the function of a specific gene is knocked out using dsRNA. RNAi can achieve sequence specific degradation of its homologous mRNA sequence. The dsRNA is cleaved into a siRNA (small interfering RNA) duplex by a dicer. siRNA have complementary base pairs to target mRNA, and form a complex with RISC leading them to the target mRNA. The small RNA binds to the mRNA, inducing its cleavage by RISC and thus preventing mRNA from producing a protein. The selective and robust silencing of a gene by RNAi makes it a valuable research tool. With the capability to design dsRNA for every gene and the availability of complete genome sequences, it is possible to perform genome wide screens using RNAi. The complete genome analysis of C. elegans [81] by RNAi has proven that it is an ideal tool for high throughput functional genomics. Since the success of the genome wide RNAi screen of $C$. elegans, a number of functional genomics studies based on RNAi screening have been conducted in Drosophila tissues and human cell cultures. Genome wide analysis has been performed on notch signaling [82], self-renewal of neural stem cells [83], and muscle morphogenesis in Drosophila [25]. A collection of Drosophila melanogaster RNAi transgenes for $88 \%$ of the predicted protein-coding genes $(22,247$ lines) is provided by the Vienna Drosophila RNAi center [84]. RNAi stock is also available from the Transgenic RNAi Project (TRIP) of Harvard Medical School [85] and the National Institute of Genetics (NIG-FLY: 8,182 lines) [86]. With the availability of RNAi lines, it has become faster and easier to design a model for studying various biological processes in Drosophila.

As mentioned previously, targeted gene silencing by RNAi can be achieved by using it with a UAS-GAL4 system (Figure 7). In this approach GAL4 controls two responder transgenes: UAS-dsRNA transgene and UAS-FP (fluorescent protein) 
transgene. UAS-dsRNA expresses the dsRNA that knocks out the function of a specific gene. A GAL4 driver also activates the UAS-FP transgene that is present on the same chromosome. Thus, the expression of a fluorescent protein would indicate the silencing of a gene that corresponds to the RNAi. A similar approach has been used to silence genes in muscles in this work. The protocols for genetic screens in our study are discussed in the next section.

\subsection{Targeted Genetic Perturbation in Muscles}

We used the UAS-GAL4 system in combination with RNAi, as explained in previous section, for muscle specific silencing of genes. For visualization of both muscles and myonuclei, we used a reporter line with two fluorescent proteins. We will discuss the details of Drosophila strains and crossing protocols in this section.

\subsubsection{Drosophila Strains}

The Drosophila strains used in this study are

1. Reporter line with GFP and mKO:

MHC-tauGFP/FM7-GFP; Mef2-Gal4, UAS-Histone 2Av-mKO/ TM6B Tb

2. UAS-IR (Inverted repeat) transgenic RNAi lines from TRiP at Harvard Medical School (NIH/NIGMS R01-GM084947):

UAS-GeneX-RNAi

All Drosophila strains were kept either at $25^{\circ} \mathrm{C}$ or $18^{\circ} \mathrm{C}$. Mef2-GAL4 was used as a muscle specific driver. MHC-tauGFP was used to label muscle cytoplasm and UASHistone 2Av-mKO to label nuclei. The silencing of GeneX using RNAi expression was controlled by the UAS-GAL4 system. In a cross between the reporter line and UASGeneX-RNAi line, expression of mKO in the muscle cells of the progeny indicates that the RNAi was also expressed.

\subsubsection{Preparing Crosses}

The reporter line was crossed to the UAS-RNAi lines in order to examine the muscle phenotype. Virgin females from reporter line were collected. The virgin 
females were kept in separate vials at $25^{\circ} \mathrm{C}$. If after 2-3 days, no larvae were observed in the vial, the females were used for crosses. 10 female virgins were crossed to 10 males from RNAi line. All crosses were kept at $25^{\circ} \mathrm{C}$. Every $2-3$ days the flies were transferred to new vials to obtain multiple cultures of similar age. The mechanism of expressing fluorescent proteins in muscles using UAS-GAL4 system is shown in Figure 8.

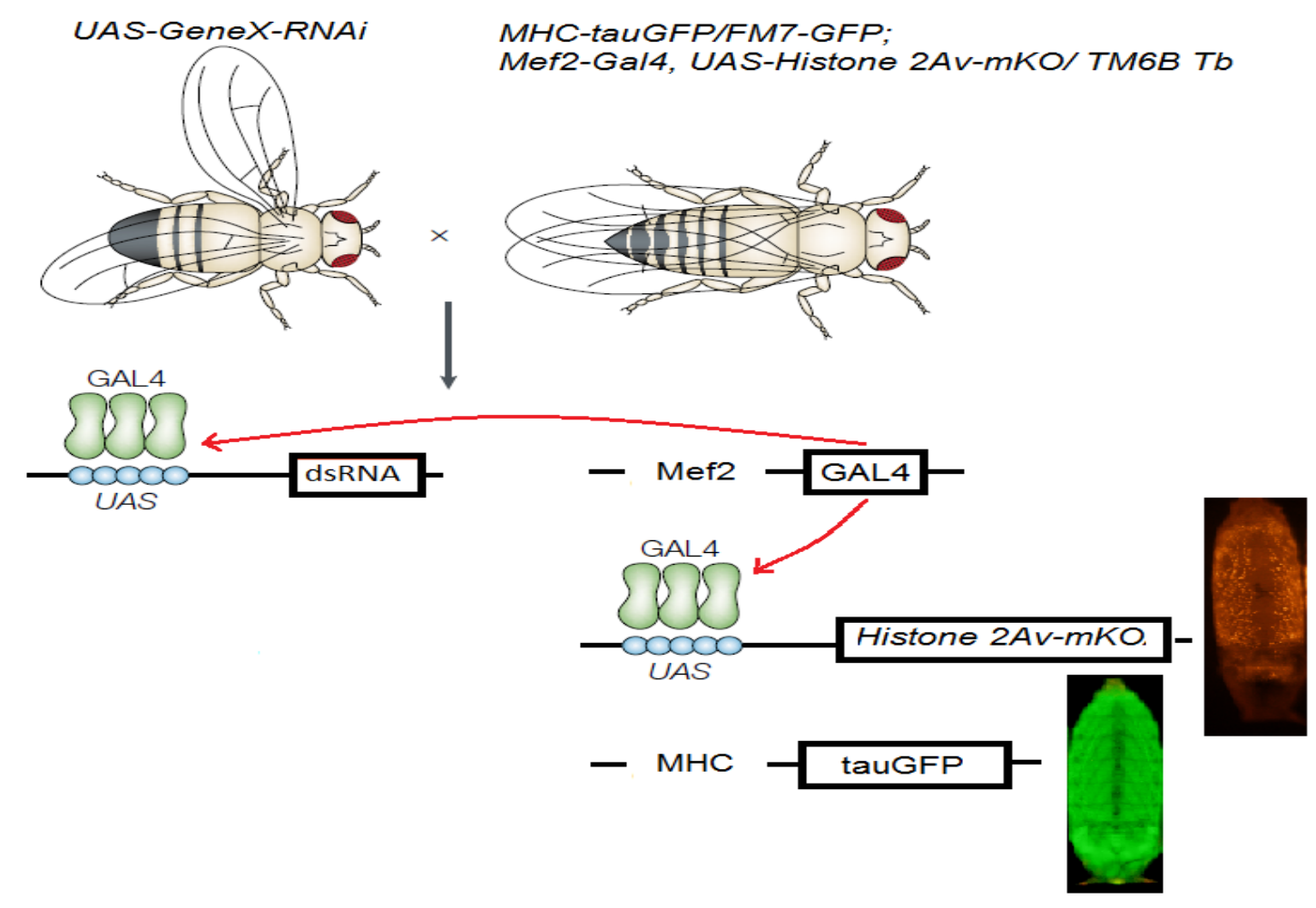

Figure 8. Muscle specific expression of two fluorescent proteins using UAS-GAL4 system.

\subsubsection{Specimen preparation for imaging}

Larvae were collected during the white prepupal stage for imaging. They were washed with water to remove the fly food from their surface. This helped in reducing the background fluorescence. Once washed, they were observed under macrozoom microscope to select larva expressing RNAi. For phenotypic analysis, we examined the non-Tubby (non-Tb), mKO expressing flies. 


\subsection{Phenotypic Analysis}

Next, we analyzed the effects of silencing of genes on the development of Drosophila; most importantly, muscle remodeling during metamorphosis. Silencing of genes can result in different types of phenotypic affects, for example, lethality during larval stage, enlarged muscles during pupal stage or flightlessness. Therefore, before analyzing muscles in detail, we categorized the genetic perturbations based on a preliminary phenotypic screening (level-1).

\subsubsection{Level-1 screen}

At level-1 screening, we either visually identified the phenotypes or use macro zoom fluorescence microscope. We collected images of muscle between large intervals of time which made it faster to analyze the phenotypic changes in muscles. This is an efficient system to identify genes of interest which require further analysis using confocal microscope. For level-1 screening, 20-30 samples (section 2.2.3) were selected and placed on a petri dish for lethality test and muscle morphology analysis.

\subsubsection{Macro zoom fluorescence microscope}

We used Macro Zoom Fluorescence Microscope, also called stereo microscope (Figure 10a) for qualitative analysis of muscle phenotypes. Snapshots of 20 samples from each RNAi cross were taken. The images were collected every 12-24 hours. A DP73 Olympus camera mounted on a MVX10 Olympus Macro Zoom Fluorescence microscope was used to capture the images. The GFP filter was used to view the fluorescent muscles. The live feed from the camera was observed on CellSens Standard software. Image of four pupae was taken in one frame at 1.6x magnification. The acquired RGB images were $2400 \times 1800$ in size at 24 bit depth.

\subsubsection{Lethality test}

1. Larval lethality: We observed the movement of 3rd instar larva on the surface of vial. If they did not move when agitated, then we checked whether pupariation has started. Pupariation is preceded by shortening of larva, eversion of anterior spiracles and 
hardening of the cuticle. If pupariation did not occur after emergence of 3rd instar larva then the phenotype is larval lethal.

2. Prepupal lethal: Prepupae fail to progress to pupal stage. In Drosophila prepupa, head eversion occurs approximately 12 hours APF, which marks the onset of the pupal stage.

During head eversion the body moves towards the posterior end of pupa and massive muscle displacement is observed. We collected equal number of mutant and wild type prepupae (20) in a petri dish and observed them under a stereo microscope.An absence of head eversion indicates developmental arrest during prepupal stage.

3. Pupal lethal: Head eversion indicates the start of pupal stage. The body segments i.e. head, thorax and abdomen starts to form after head eversion. Histolysis of DEOMs starts at approximately 10 hours APF. At approximately 20-24 hours APF, most of the DEOMs are destroyed. During histolysis, the broken down muscles can be seen throughout the body. The DIOMs survive and form adult muscles. During late pupal stage, eye color darkens and bristles on thorax and abdomen become visible. Eclosion occurs at around $110-120$ hours APF at $23^{\circ} \mathrm{C}$. We examined these phenotypes in the selected pupae to confirm the lethality stage. If the sample undergoes developmental arrest within 30 hours APF, then the phenotype is early pupal lethal. However if the pupa continued to develop but did not eclose, then the phenotype is late pupal lethal. By observing pupa under stereo microscope at every 12 hour interval, we determined whether developmental arrest occurred during early pupal stage or late pupal stage. We observed that the time of eclosion varied depending on the temperature during development of pupa. The adult fly eclosed around 90-100 hours APF at $25^{\circ} \mathrm{C}$ that is lower than the eclosion time at $23^{\circ} \mathrm{C}$.

\subsubsection{Flight analysis}

For flight analysis, 5 to 10 flies were placed in a vial. The vial was gently tapped to observe the flight events.

\subsubsection{Muscle morphology analysis}

The muscle morphology during each stage was observed as explained before. The list of phenotypes studied is given in Table 2 . 


\begin{tabular}{|c|c|}
\hline $\begin{array}{l}\text { Development } \\
\text { Stage }\end{array}$ & Phenotype test \\
\hline Early Prepupa & $\begin{array}{l}\text { - Whether dorsal internal and external oblique } \\
\text { muscles were present in each abdomen segment? } \\
\text { (Figure 9a) }\end{array}$ \\
\hline Late Prepupa & $\begin{array}{l}\text { - Whether prepupal to pupal transition started? } \\
\text { (Figure 9b) } \\
\text { - Whether histolysis of dorsal abdominal muscles } \\
\text { started? }\end{array}$ \\
\hline Early Pupa & $\begin{array}{l}\text { - Whether eversion of head was successful? } \\
\text { - Whether external oblique muscle cells were } \\
\text { histolysed completely? (Figure 9c) }\end{array}$ \\
\hline Late Pupa & $\begin{array}{l}\text { - Whether the abdomen muscles showed any } \\
\text { abnormal shape? } \\
\text { - Whether pigmentation (Bristles/eye color) was } \\
\text { observed? }\end{array}$ \\
\hline Adult & Flight test \\
\hline
\end{tabular}

Table 2. List of phenotypic tests for different developmental stages during Drosophila metamorphosis. 

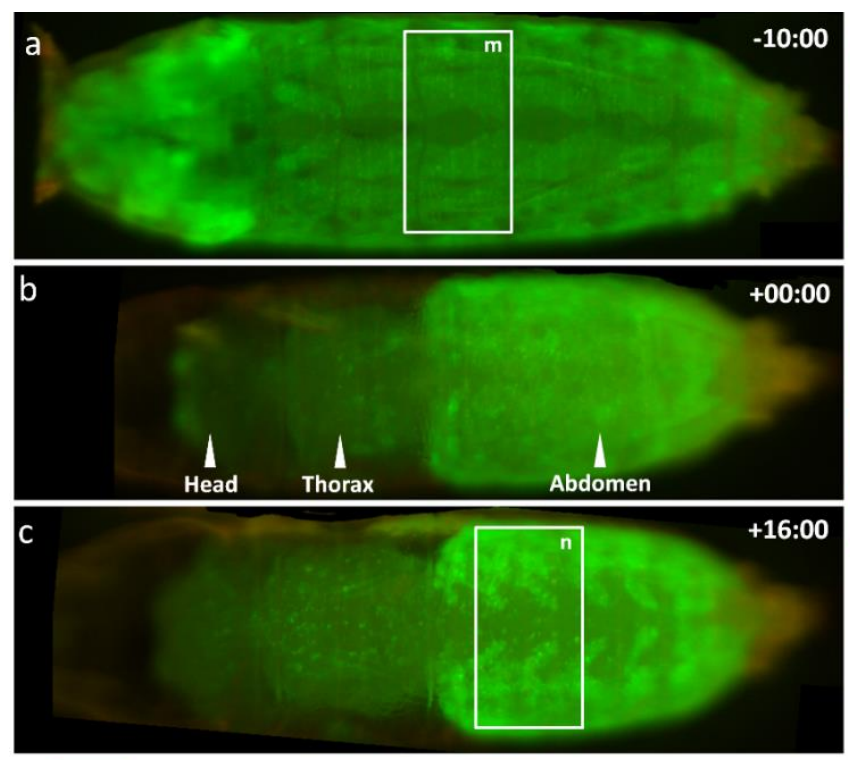

- External Oblique Muscle Internal Oblique Muscle

Missing External Oblique Muscle

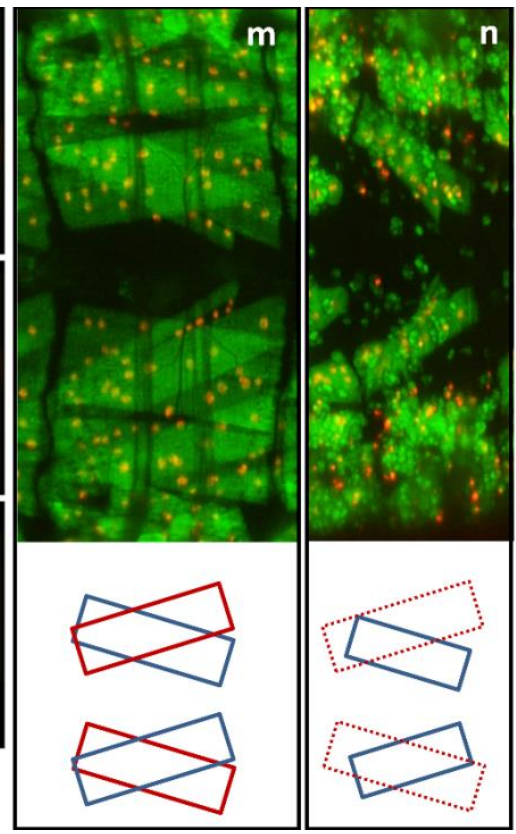

Figure 9. Muscle phenotype at different stages of development during metamorphosis.

a) Prepupal stage. Enlarged view of dorsal external and internal oblique muscle is shown in (m). b) Formation of head, thorax and abdomen during head eversion. c) External oblique muscles undergo histolysis during head eversion. They are completely destroyed by early pupal stage as shown in (n).

\subsubsection{Level-2 screen}

Level-2 screening was for genetic perturbations which were found to affect the muscle morphology and myonuclear distribution during level-1 screening. In level-2 screening, we imaged the muscle cells at higher resolution and more frequently using confocal microscope. For confocal imaging, we selected 10-12 samples of each RNAi expressing prepupa. They were placed on an uncoated $32 \mathrm{~mm}$ diameter glass bottom dish (MatTek). The dorsal side of the prepupa was towards the bottom of the dish. On one dish approximately 30 prepupa were arranged in 3 rows. The prepupae were embedded with CyGEL to restrict their movement during imaging. A wet tissue was kept around the specimens to maintain humidity level during development. 


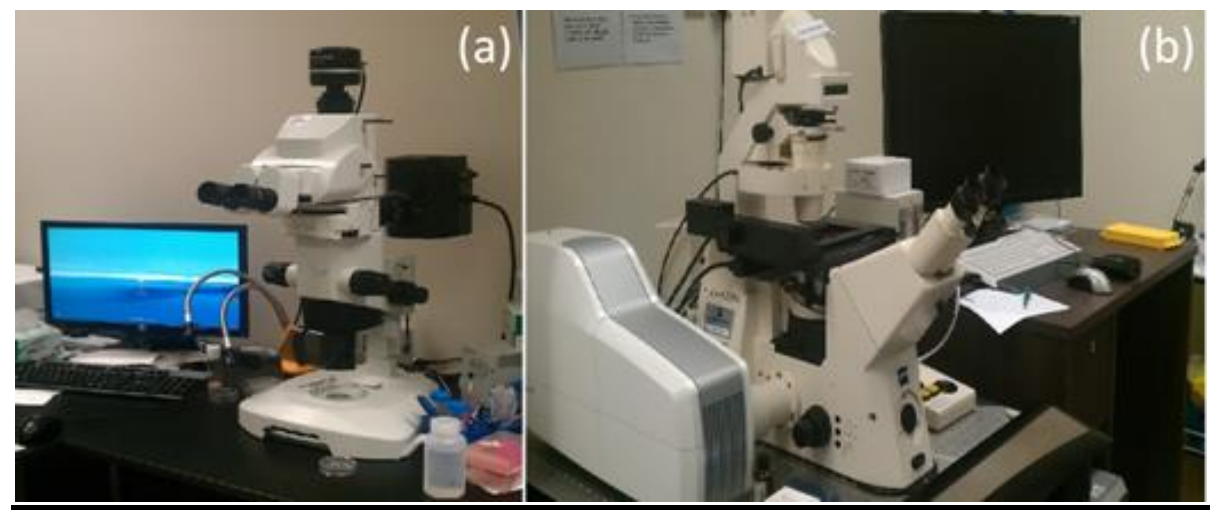

Figure 10. a) Macro zoom fluorescence microscope b) Line scanning Zeiss LSM 5 Live microscope.

\subsubsection{Confocal microscopy}

We used line scanning Zeiss LSM 5 Live microscope (Figure 10b) to perform live cell imaging of Drosophila pupae. We collected images at multiple focal planes and multiple time points for each sample. Zen 2008 software was used to configure the settings of the confocal microscope. We acquired the images with the following settings; 488 laser diode (GFP), 10x objective magnification (EC Plan-Neofluar 10x/0.30 M27), Pin hole size 16.6 $\mu \mathrm{m}$ and frame speed 2 FPS. The refractive index correction was set at 1 for air. For individual sample the $\mathrm{z}$ stack and location setting was done separately. The live images from LSM were viewed directly on ZEN software. The first and last slice of the $\mathrm{z}$ stack was chosen by adjusting the fine focus. The optimum number of slice was calculated based on the distance between two $\mathrm{z}$ slices chosen by the user. The number varied from 30 to 40 slices. Because single slide can accommodate around 28-30 samples, the confocal was configured to collect images at multiple locations. The location of each sample was marked by adjusting the stage at appropriate position on top of the sample. The marked locations were loaded into a multi-location imaging macro installed in ZEN 2008. This macro was also used to set the interval between consecutive image collection and total duration of the run. For our dataset, images were taken at 30 minute interval. The duration of imaging varied from 3 to 5 days, depending on the temperature during imaging. The confocal images (1024x1024, 8 bit) were stored in LSM format (Carl Zeiss). One lsm file contains 3D stack images from one sample at one time point. Further processing (concatenation) is required to view every time points of a sample together. 


\subsection{Summary}

In this chapter, the detailed protocol of genetic screens and image acquisition is provided. The proposed methodology enables us to examine the muscle phenotypes caused by genetic perturbation using imaging techniques. The contributions are summarized as follows:

1. A system for conducting live cell time lapse imaging of Drosophila pupae has been developed. Its capability to image time lapse in vivo images helps in studying the transient phenotypes of the muscles. The proposed methodology visualizes both muscle cells and nuclei using two markers at multiple z-planes.

2. A two level-phenotypic screening protocol has been proposed. Since confocal microscopy is not a high throughput technique, analyzing samples that do not show any muscle phenotype would be a waste of resources and time. Thus, a preliminary screening to short list genes with the muscle phenotype was applied. 


\section{Chapter 3 FMAj for Time Series}

Analysis of Drosophila Muscles

Time series in vivo imaging of Drosophila muscles for large durations has not been conducted. Two major problems that this study attempted to address are discussed below:

1. The imaging of a large number of genetic perturbations for 4-5 days produced sizeable amounts of image data. Image acquisition at $30 \mathrm{~min}$ intervals produced data of approximately 18-20 GB per sample. Considering each genotype and their replicates, the amount of data produced is many terabytes. Managing and analyzing such large data requires the automation of image processing.

2. Drosophila abdomen muscle images are highly complex and few muscle processing algorithms (segmentation, muscle features) are available [77]. In recent years, many excellent non-commercial software packages for generic image analysis have been developed, including ImageJ [87], Fiji [88], CellProfiler [89], BioImageXD [90]. However, these tools do not provide image analysis algorithms for Drosophila muscle specific problems like muscle and non-muscle region classification and myonuclear spatial pattern analysis. Another problem with these tools is the lack of integrated image/data storage, image annotation, processing and visualization to facilitate analysis of very large number of images. If the above mentioned components become part of a pipeline with automated flow of data (features, contours, image metadata) between them, then the muscle image analysis will be faster, reproducible and more accurate with least manual intervention. Although, the tools like CellProfiler and BioImageXD have application to build pipelines but only using analysis method already implemented in them. Therefore, it became necessary to create a muscle specific image analysis pipeline/tool by extending existing image analysis libraries.

I decided to develop a custom software tool that could implement algorithms for pre-processing, segmentation, feature generation and statistical analysis of muscle 
images, and managing the data through relational database management, resulting in a minimal amount of manual data processing. I designed a custom image analysis tool (FMAj) based on ImageJ. FMAj uses the image processing libraries of ImageJ as a foundation for developing advanced algorithms. In this chapter, the details of time series analysis of muscles and the flow of data between FMAj and database is discussed. Figure 11 shows a schematic diagram of the quantitative time series analysis of muscle cells. 


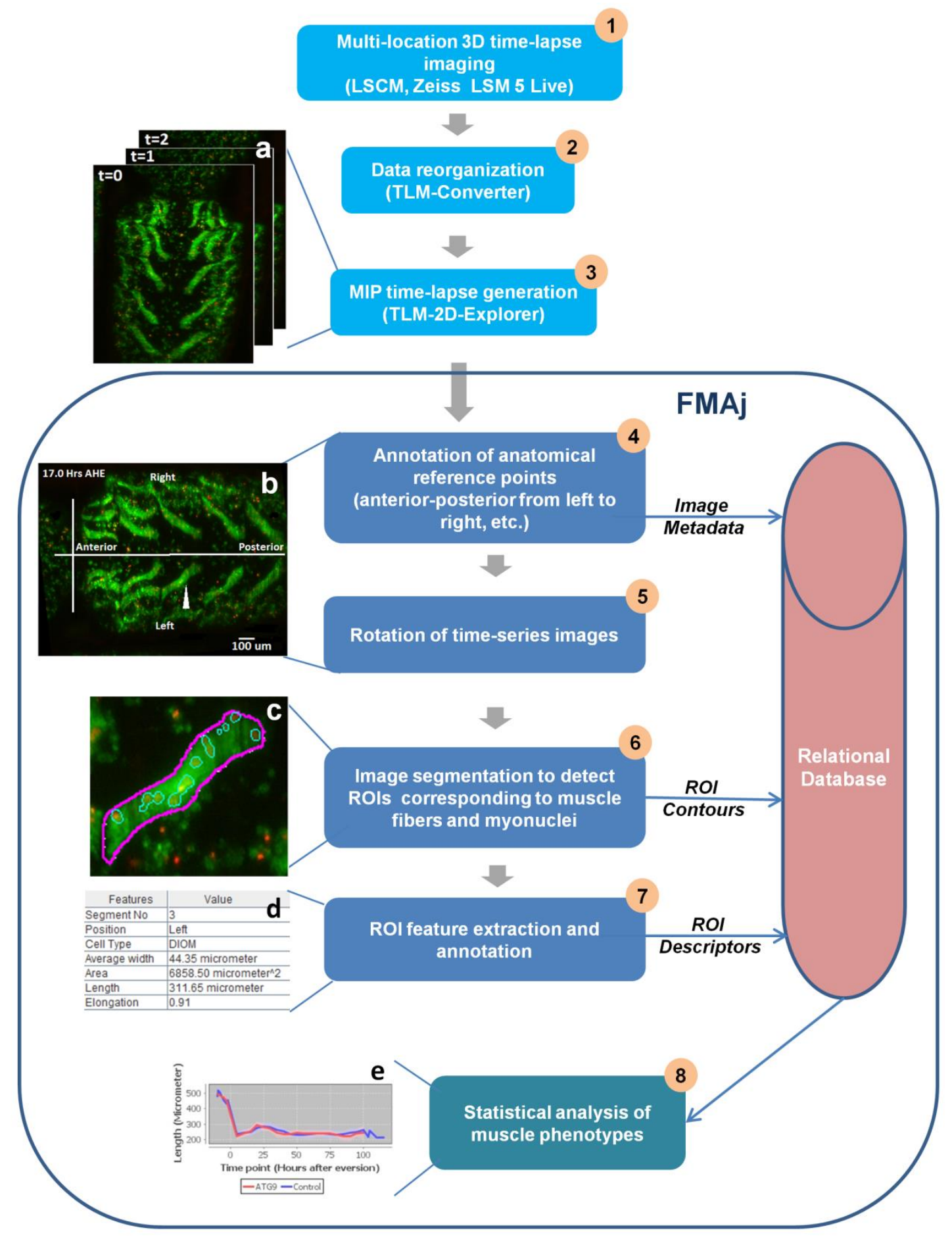

Figure 11. Pipeline for quantitative time series phenotypic analysis of muscles. 


\subsection{Data Formatting and Pre-Processing}

FMAj processes 2D projection instead of 3D stacks for high throughput analysis of time series images. Thus, the images from confocal microscope are pre-processed before they could be used in the tool. The images obtained from microscope are in LSM format. The microscope creates one LSM file containing 3D stack per sample per time point. The files from each sample were concatenated using the TLM software [91]. I used Image Cytometry Standard (ICS) format to store the images. Image files can be opened and viewed in ImageJ [87] using LOCI plugin [92] (Figure 12a). For the purpose of time series analysis, the 3D stack of ICS images was further processed to obtain their maximum intensity projection (MIP). An in house software tool was used to produce MIP from the ICS files[93]. It produces MIP in tiff format for each time frame.

\subsection{Image Analysis Workflow of the FMAj Tool}

FMAj tool consists of three modules: annotation, segmentation and feature extraction, and quantitative phenotypic analysis. Each module performs a specific type of task as their names imply and is dependent on the data from other modules. Another important feature of this tool is the incorporation of a MySQL database to store the segmented regions of interest (ROIs) along with extracted features. Next, I'll will discuss the role of each module and their interactions with each other and MySQL database.

After starting the application, the user establishes a database connection and selects the root folder of the microscopic images. Once the database is online, the tool automatically downloads the experimental information, such as gene names, stock ids, muscle type, developmental stages and the imaging protocol from the MySQL database. Initially, metadata about image acquisition are extracted from raw image files, while biological details are entered into the database by an expert user. Input images of FMAj are time-series MIPs in RGB format containing two color channels; with green representing the cytoplasm and red the nuclei of muscle fibers (Figure 12a). The tool provides options to view both channels together or separately. As mentioned before, 
FMAj tool consists of three modules which perform three sequential tasks. The first module captures experimental metadata which are either derived from the images or via manual annotation by the user. An important user input is the definition of the onset of head eversion, which serves as the main reference point (time point $=0$ hours) for comparing datasets. The second module performs segmentation of muscle cells and nuclei. Features of detected ROIs are either calculated (e.g. shape, pattern) or annotated by the expert user (e.g. cell nomenclature). The third module performs comparative phenotypic analysis, such as comparing the cell morphology between control and genetically perturbed cells.

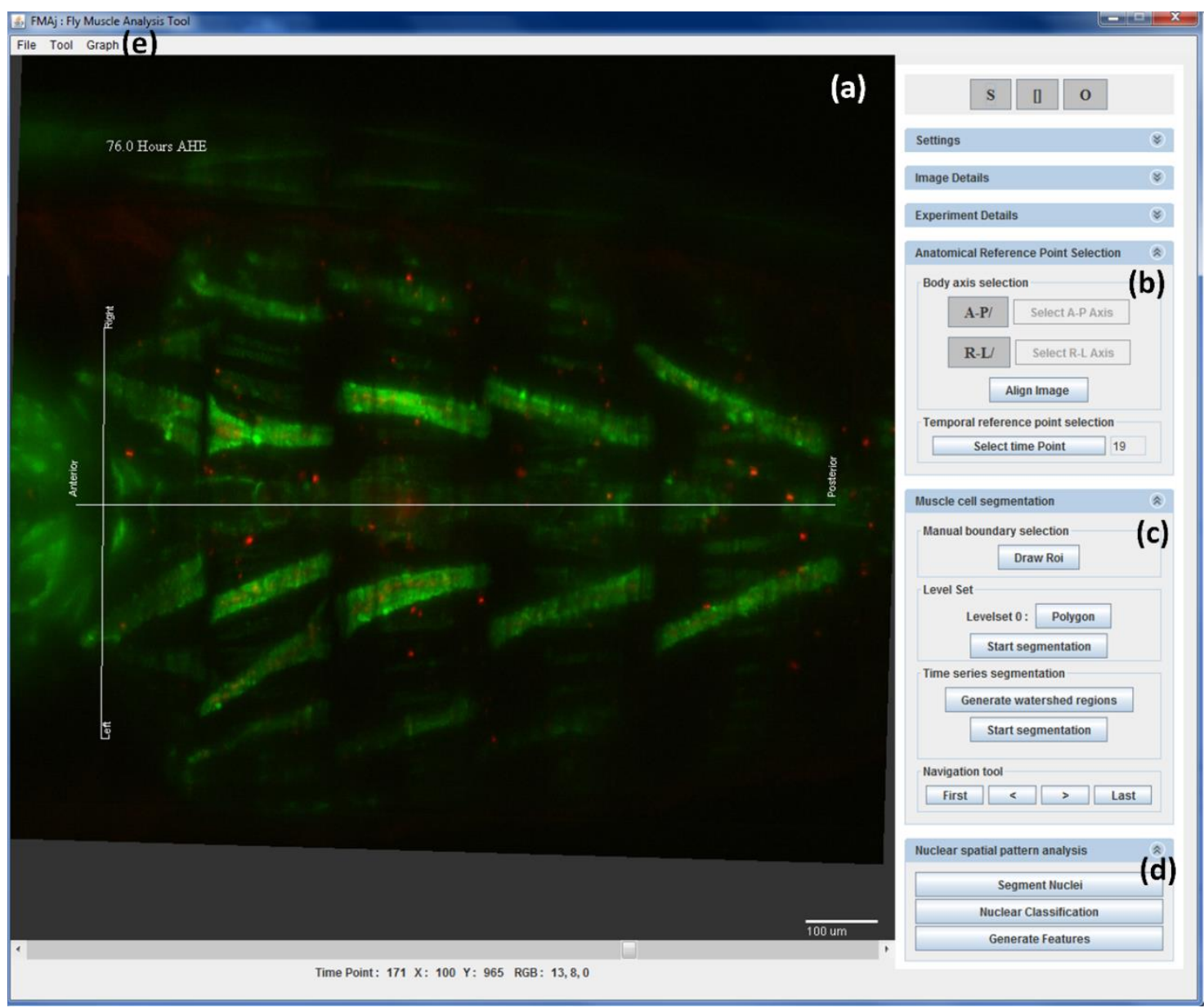

Figure 12. Screenshot of the FMAj user interface.

a) Panel to view time series stack. b) Application for annotation of time series images. c) Application for segmentation of muscle cells. d) Nuclear spatial pattern analysis application. e) Application for graphical visualization of time series data. 


\subsubsection{Annotation of time-series images}

To facilitate the objective comparison of image sets of different samples, a robust spatial and temporal reference system was established (Figure 12b). Spatial registration is achieved by interactively drawing a left-right symmetry axis along the midline and rotating the time-lapse image stack so that the anterior to posterior axis of the pupa is oriented horizontally from left to right. In addition, the user draws a second line that demarcates the boundary between thorax and abdomen. These reference lines assist in the visual comparison of different samples. The onset of head eversion occurs approximately 12 hours after puparium formation and was used for the temporal alignment of different samples, thus enabling the comparison of equivalent frames in different time-lapse datasets [77].

\subsubsection{Segmentation and feature extraction}

\subsubsection{Morphological analysis of muscle cells}

The goal of muscle segmentation is to quantify the morphological changes of persistent abdominal muscles throughout metamorphosis. In the early pupal stages right after head eversion, muscles are difficult, if not impossible, to segment using automated methods due to large amounts of debris created by the histolysis of obsolete muscles. Therefore, I decided against an automatic approach for muscle detection. Instead, I designed three ways for generating muscle boundary (Figure 12c).

1. Drawing the muscle boundary manually, especially during early pupal stages.

2. Using a semi-automated active level set method [94] for segmentation of muscle cells. In the first step, the user draws one or more polygons around muscles of interest in one or more frames. In the second step, the polygons serve as initial level sets for the subsequent curve evolution, which is performed by an ImageJ level set plugin [95]. The contour evolution is controlled by an edge-based constraint that applies grey value and curvature penalties to prevent the leakage of contours into low contrast regions. For muscle cell segmentation, contour evolution is restricted to the inside of manually drawn polygons. If the output of the level-set method is not satisfactory, the user can delete the ROI and manually redraw its boundary. 
3. Third option available is the watershed based time series segmentation designed specifically for segmentation of muscle cells. This method uses the muscle boundary (generated manually or using level set) at one time point to segment muscles in subsequent time points automatically. I'll will discuss about this segmentation algorithm in detail in chapter 4.

For each segmented muscle in a particular frame, the user assigns a unique description based on 4 criteria; (1) the cell type (e.g. DEOM or DIOM), (2) the body part (e.g. thorax or abdomen), (3) the segment (e.g. abdominal segment 3) and (4) the lateral position (left or right hemi-segment). To improve productivity, lateral positions can be inferred from the midline, while tracking of overlapping ROIs provides the options to automatically propagate annotations to other subsequent frames. Correspondences between cells in adjacent time points are established using a nearest neighbor search on the basis of minimum distance between centroids. To avoid incorrect tracking, a maximum distance $D$ between centroids of adjacent frames is used as a constraint. $D$ was estimated based on the maximum movement of muscle cells observed in 30 minute intervals. Highlighting different cell types with different contour colors helps to identify annotation errors that can be manually corrected by the user.

The difference in muscle shape and size between mutants can be quantified by comparing various morphological features extracted from muscle cell contours. The collection of features available in FMAj are average diameter, area, length, elongation, orientation and extent [96]. Average diameter, area and length quantify the size of muscle cell. Average diameter is calculated by measuring the width of muscle cell at multiple points along its skeleton [97], which is equivalent to the medial axis of the muscle cell. Elongation and extent quantify the shape of the muscle cells. Elongation, as the name suggests, quantifies the elongation of muscle cells and is defined by the ratio of length of minor and major axis. Extent, on the other hand, measures whether the muscle cells are rectangular in shape. Extent is the ratio of the area of muscle cell and the area of its bounding box.

\subsubsection{Spatial pattern analysis of myonuclei}

Muscle cells are multinucleated. In order to study the distribution of nuclei inside the muscle cells, locations of nuclei are required. Since the images were recorded at a 
relatively low resolution using a 10x objective, it was difficult to identify individual nuclei. Hence, instead of identifying the centroids of all nuclei, I detected the regions (or clusters of nuclei) of muscle fiber. FMAj segments nuclei for all the time points where muscle boundary is available and calculates the spatial pattern features (Figure 12d). The details about nuclear segmentation and the spatial pattern features used are given in chapter 5 . The boundaries of nuclei can be viewed as contours within muscle ROIs in FMAj (Figures 11c).

\subsubsection{Quantitative phenotypic analysis}

\subsubsection{Data browsing}

FMAj allows the user to view images, annotations and processed data. To detect interesting phenotypes, spatially and temporally aligned datasets can be viewed side by side. Annotations such as boundaries can be projected on top of images.

When the user opens a multi-page TIFF image stack in FMAj, the tool retrieves image metadata like width, height, physical pixel size and bit depth. The user can view the image acquisition settings extracted from LSM metadata like magnification, laser wavelength and pinhole size. The user can also upload temperature and humidity readings collected using a temperature logger device from a spreadsheet to the database and visualize them in the graphical form.

\subsubsection{Statistical analysis}

To compare muscle phenotypes of different RNAi genotypes, I segmented muscles in abdominal segments 3-5 of 10 pupae per genotype at 5 hour intervals. With one muscle per hemi-segment, the statistical analysis comprised 10-20 muscles per genotype and time-point. To compare the morphology of different genotypes at equivalent times in development, I performed the non-parametric MannWhitney U test in FMAj with help of the Java Statistical Classes (JSC) library [98]. Significance values (p) were determined using a non-parametric test because of low number of samples per population (10-20) and unknown distributions. The MannWhitney U test statistics can be viewed in graphical form using the phenotype comparison tool. For ease of visualization, p-values are plotted in a $-1 * \log _{10}(\mathrm{p}$-value) scale. 
Similarly, for comparing the distribution of nuclei in different RNAi genotypes, I segmented nuclei for the available muscle boundaries and calculated their spatial pattern features. The Mann-Whitney U test was also used for testing statistics of spatial pattern features.

\subsubsection{Graphical visualization of time-series data}

FMAj has built-in charting functions, such as line plots, that can be used to visualize and compare the dynamics of cellular features (Figure 12e). FMAj uses jFreeChart library to generate graphs [99]. The user interface allows users to select the genotypes, muscle types and shape features (Figure 13-a1). When comparing different populations, mean or median values along with their error margins (standard deviations, 25-75\% percentiles) can be plotted. Significance values can be displayed underneath the charts of cellular features (Figure 13-a1). Environmental parameters like temperature and humidity can also be plotted (Figure 13b). An application to view and compare the muscle contours between two samples is also available in FMAj (Figure 14).

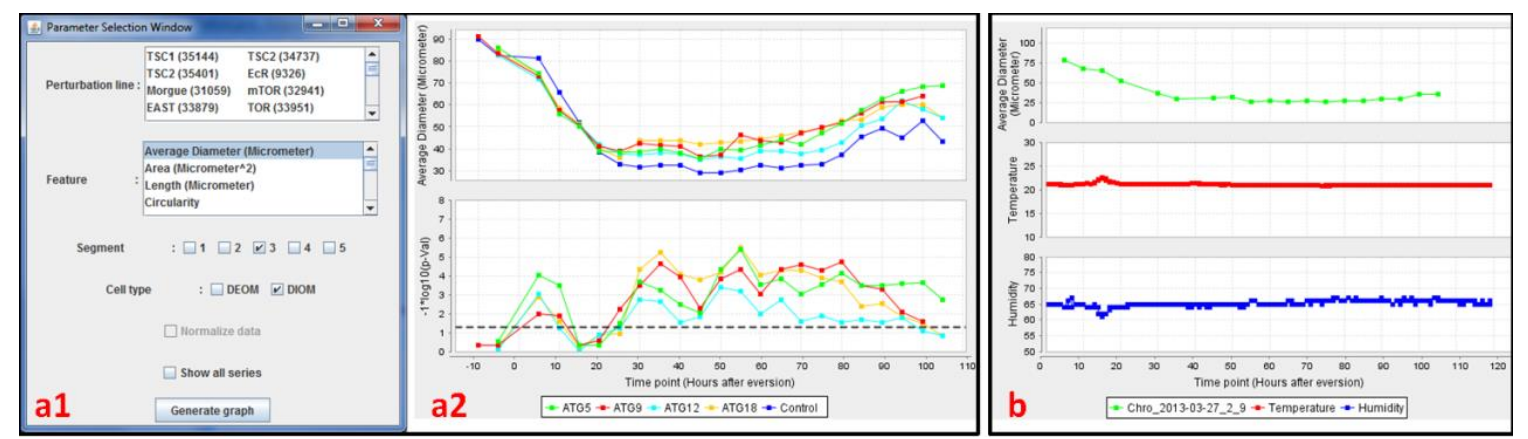

Figure 13. Quantitative phenotypic analysis using FMAj.

a) Application to compare muscle features. User has to choose from multiple options like perturbation line, feature and muscle type, (a1) in order to generate a graph (a2). b) Application to check whether temperature/humidity is responsible for any change in muscle morphology. 


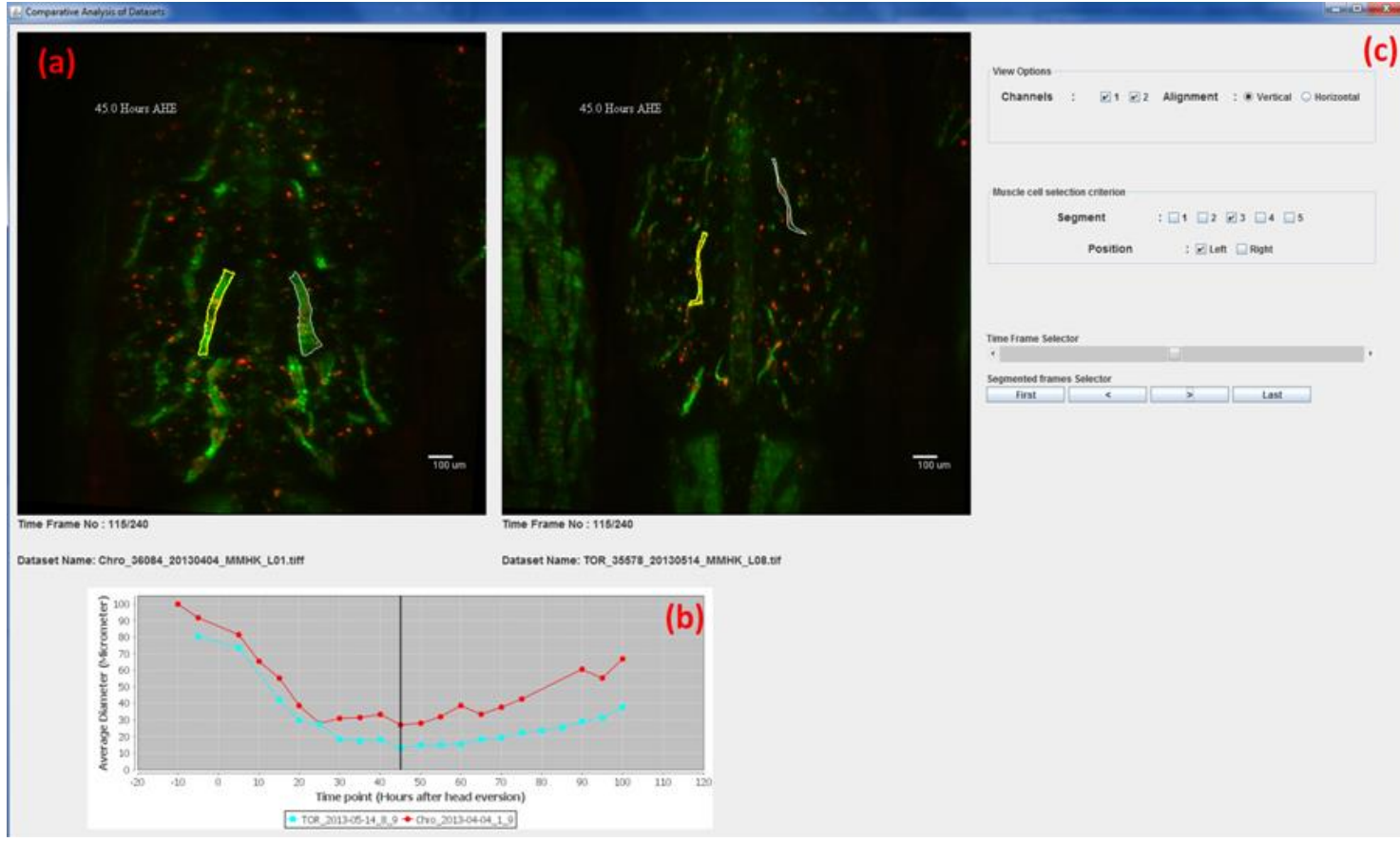

Figure 14. Screenshot of an FMAj application to view and compare the muscle contours between two samples.

a) Two samples chosen by the user are shown with overlaid muscle contours. b) A user selected feature is plotted for the muscles in yellow contour (a). c) Navigation tool and options to plot features of other muscles.

\subsection{Relational Database Management}

To improve data handling efficiency, I created a relational database using MySQL and connected it to FMAj. Figure 15a shows the general dataflow between FMAj and the database. MySQL is an open source database which ensures data integrity and is very efficient in querying large amount of data [100]. The dependencies and relationships between the entities of our experimental data were modeled in the relational database tables (Figure $15 b$ ). 
(a)

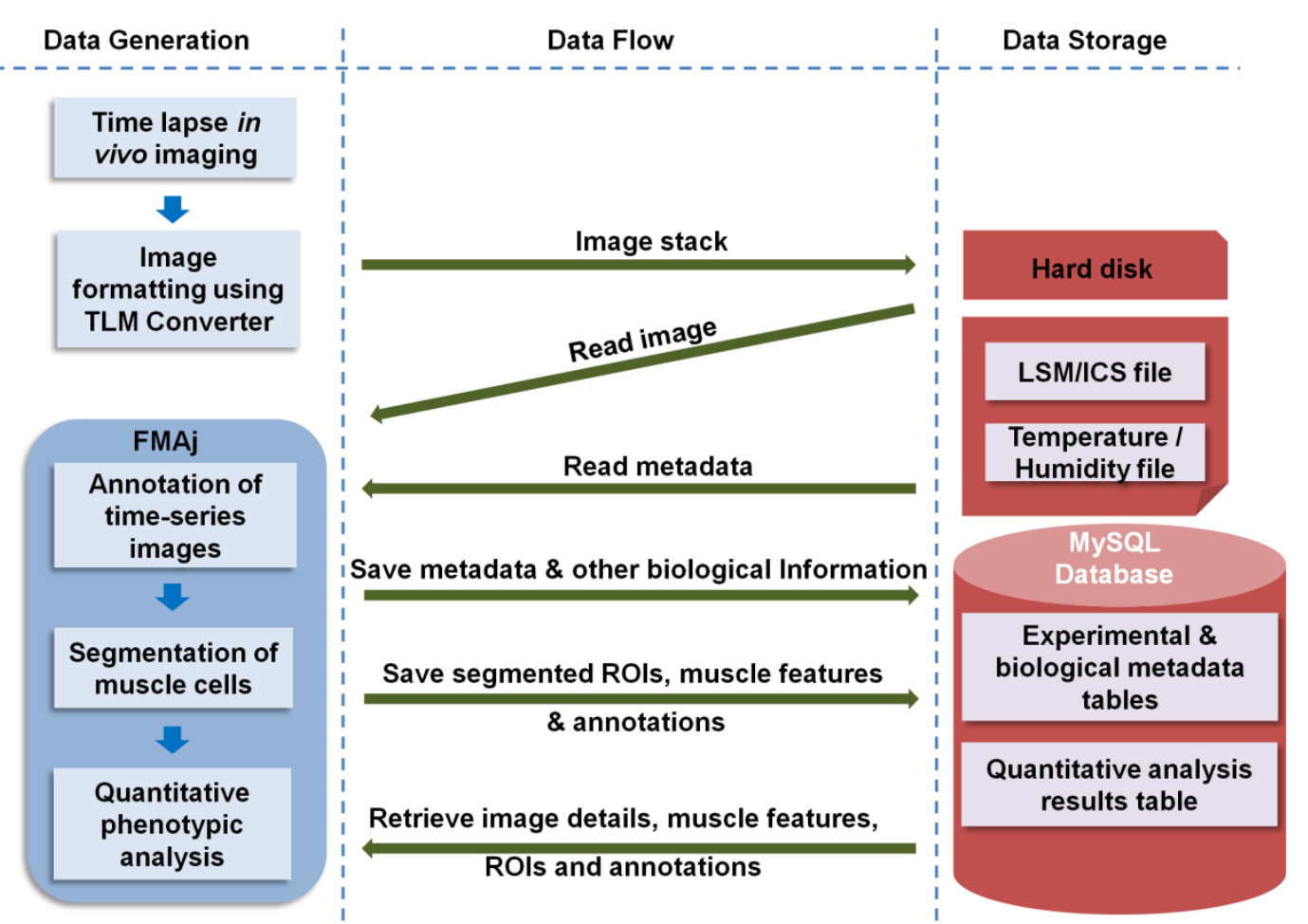

(b)

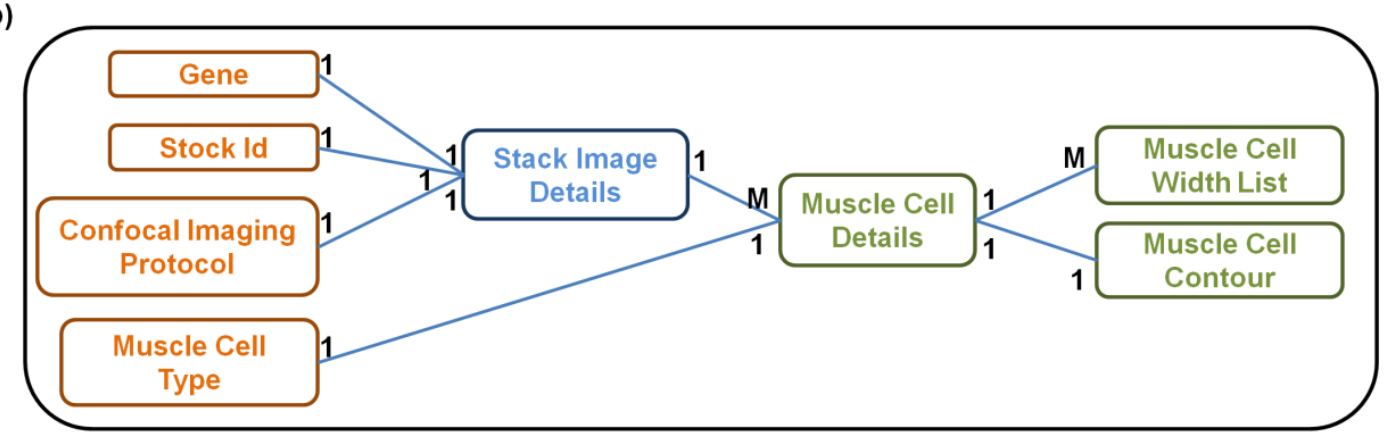

Figure 15. Relational database

(a) Schematic diagram of the dataflow between FMAj and the relational database. The forward arrows indicate storage of data into MySQL while backward arrows indicate retrieval of data from MySQL. (b) Simplified version of entity relationship diagram of the database. Each block represents a table and each line indicates a relationship between tables. The notations at the end of lines define the type of relationship, such as one (1) to one (1) or one to many (M). For example, the muscle cell details table has One to One relationship with muscle cell type. Table names in orange store experimental and biological metadata, while those in green store results from image segmentation like the annotation or calculated features of muscle ROIs. 
The database is organized into two groups of tables: One for experimental and biological metadata and the other for segmentation outputs. Data collected in module 1 is stored in experimental and biological metadata tables. It contains two types of data. First, there are experimental parameters like genotypes, and imaging protocols. These data are entered directly into the database by the user. Secondly, image acquisition parameters like laser wavelength, magnification and image properties like stack name, image size, bit size, physical size of pixel are extracted from LSM image files recorded by the microscopy system. Results produced by the segmentation module 2 are stored in ROI tables. These tables store ROIs corresponding to muscle cells and nuclei as blobs. They also store the features extracted from muscle cell boundaries and nuclei. The image stacks are not stored in the database because of their large sizes. Instead, their file names and locations on the hard disk relative to a user-defined root folder are stored in the MySQL table StackImageInfoMaster. In order to make sure there is no redundancy of data, each stack can be identified by its name and a unique set of three parameters: stock id, date of acquisition and location of sample in the glass bottom dish. Module 3 of FMAj retrieves information from the database for image browsing or graphical comparison of different samples based on specific criteria of query.

\subsection{Summary}

I developed the FMAj software tool for the quantitative characterization of muscle phenotypes in time series images. To effectively perform comparative phenotypic profiling of muscle development, various modules i.e. image visualization, annotation, muscle segmentation, feature generation, structured storage by MySQL and statistical analysis were integrated. The integration of multiple tasks enhances productivity as the alternative export of ROI data and the manual processing in a spreadsheet program would be much more time-consuming. 


\section{Chapter 4 Time Series Segmentation of}

\section{Muscles in an Environment containing}

\section{Sarcolytes}

In order to quantify phenotypic changes in muscle cells, their boundaries must first be extracted. However, there are three major issues with muscle segmentation.

1. Metamorphosis lasts for 4-5 days; thus, time lapse in vivo imaging produces a large amount of images (in this study 180-240 image stacks per sample). Manually generating muscle boundaries is time consuming. Therefore, an automated or semi-automated segmentation method is required to segment a large number of image stacks.

2. The extracellular environment of muscles contains sarcolytes i.e. debris from dead muscles (Figure 16). The debris often moves close to or above muscle cell, which makes it difficult to identify boundary between muscle and debris.

3. So far, only one study has been done on muscle segmentation. In a previous study, the concept of Bayesian region-based front evolution [77] was incorporated with a semi-automated level set approach to segment muscles. With manual initialization of level sets for only one time point, this method can segment subsequent sequences of time series images. Although this technique requires less manual input, the accuracy tends to suffer when level set contour falsely identifies debris edge as muscle boundary. FMAj uses semi-automated edge based level sets for segmentation. The user draws a polygon around the muscle cell to initialize the level set and forces the contour to move inward only. Since user intervention is required for the segmentation of each cell at every time point, the results are more accurate but the process is time consuming. 

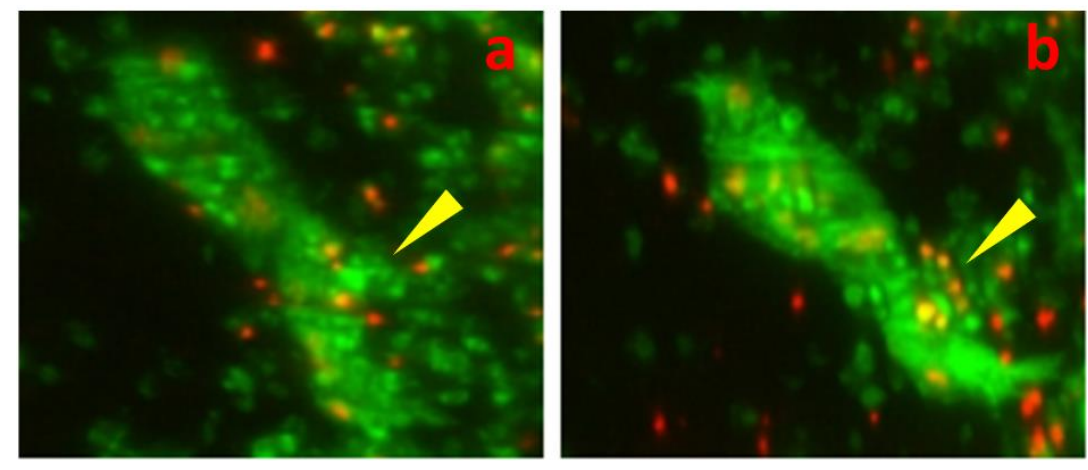

Figure 16. Sequestered muscle debris attached to the muscle cells. Yellow arrowhead indicate the muscle debris.

Therefore, an algorithm for muscle segmentation is required which can separate muscle from debris more accurately and with least manual intervention. One of the properties of persistent muscles (DIOMs) is that their movement is small between two time points and their overall shape changes slowly. This property was exploited in the new muscle segmentation algorithm to improve throughput and accuracy of segmentation. The segmentation method comprises of two steps. First, the watershed based region segmentation was performed and canny edge detection was used to generate a confidence map of edges. Secondly, the confidence map is used with chamfer distance-based feature to classify watershed regions into muscle and nonmuscle classes. In the next section, I'll explore different segmentation techniques for muscle edge extraction.

\subsection{Canny edge detection}

Canny algorithm has been used extensively for edge detection in computer vision [64]. It is a multistage algorithm and detects different type of edges reliably. The canny algorithm is divided into 5 steps:

1. The image is smoothened by applying gaussian filter. It removes the noise in the image.

2. Edge detection operator (Sobel, Roberts) is applied to the image, to calculate gradient magnitude and direction at each pixel in the image. The ridges in gradient magnitude correspond to the edges in the image. 
3. The pixels on the ridges are tracked by a process called non- maximal suppression. In other words, it tries to find the local maxima in multiple directions and assigns 0 to every non-local maxima pixel.

4. In case of a noisy edge, multiple fragments of edges are generated by previous step. To avoid that, a low and a high threshold are selected. The edge connections are tracked from an edge pixel which has gradient higher than the high threshold and tracking continues until the edge pixel's gradient value falls below the low threshold.

Comparison between the performances of canny with other edge detection algorithms is shown in figure 17 . Figure $17-\mathrm{d} 2 \& 17$-e 2 show the zero-crossing line obtained after applying Laplacian filter to the image [101]. The edges extracted using laplacian filter have been pushed inside muscles because they are affected by the low intensity regions inside the muscles. Whereas, when auto thresholding technique called isodata was used for segmenting the muscle cells, this method could not identify boundaries between muscles and debris Figure 17-d3 \& 17-e3 [102]. This technique is highly sensitive to intensity. Since at many places the intensity of debris is like muscle cells, isodata technique is unable to distinguish them. Whereas, canny detector can generate smooth curves at muscle boundaries (Figure 17-d4 \& 17-e4) [103]. Canny also generates small edges corresponding to smaller changes in intensities inside muscle and debris. Despite that, I tried to obtain the boundary of muscle by removing the smaller edges; since the performance of canny edge detector was much better than other techniques. To remove the smaller edges, Gaussian blur was applied to the image and high value of sigma $(\sigma=5)$ was used. The identification of correct edges over accuracy of the edges (i.e. edges as close as possible to the actual muscle boundary) was prioritized. After applying Gaussian blur, Gaussian derivative of the image is calculated and then the edges are isolated by non-maximum suppression. The resulting edges contain the boundaries of both muscles and sarcolytes (Figure 17a-c2, 18b). Sarcolytes have lower intensity dotted with high intensity whereas, muscle have high intensity with smaller regions of low intensity. Therefore, it's highly likely that the average of derivative will be high for a muscle edge and low for a debris/sarcolyte edge. This property was used to identify edges which belong to sarcolytes. For each canny edge $C$, the average value of Gaussian derivative $G c$ was calculated at the pixels which constitute 
the edge. The edges near muscles have higher $G c$ whereas near sarcolyte have lower $G c$. Next, kmeans was used to cluster all the edges in three groups. The cluster with edges with highest $G c$ values were labelled as muscle edges (Figure 1a-c3, 18c). Canny combined with clustering correctly identifies muscle boundaries. However, it produces open ended edges in locations where smoothening has blurred the features. Next, I'll analyze over-segmentation techniques; focusing on watershed algorithm and test how they perform on muscle images.
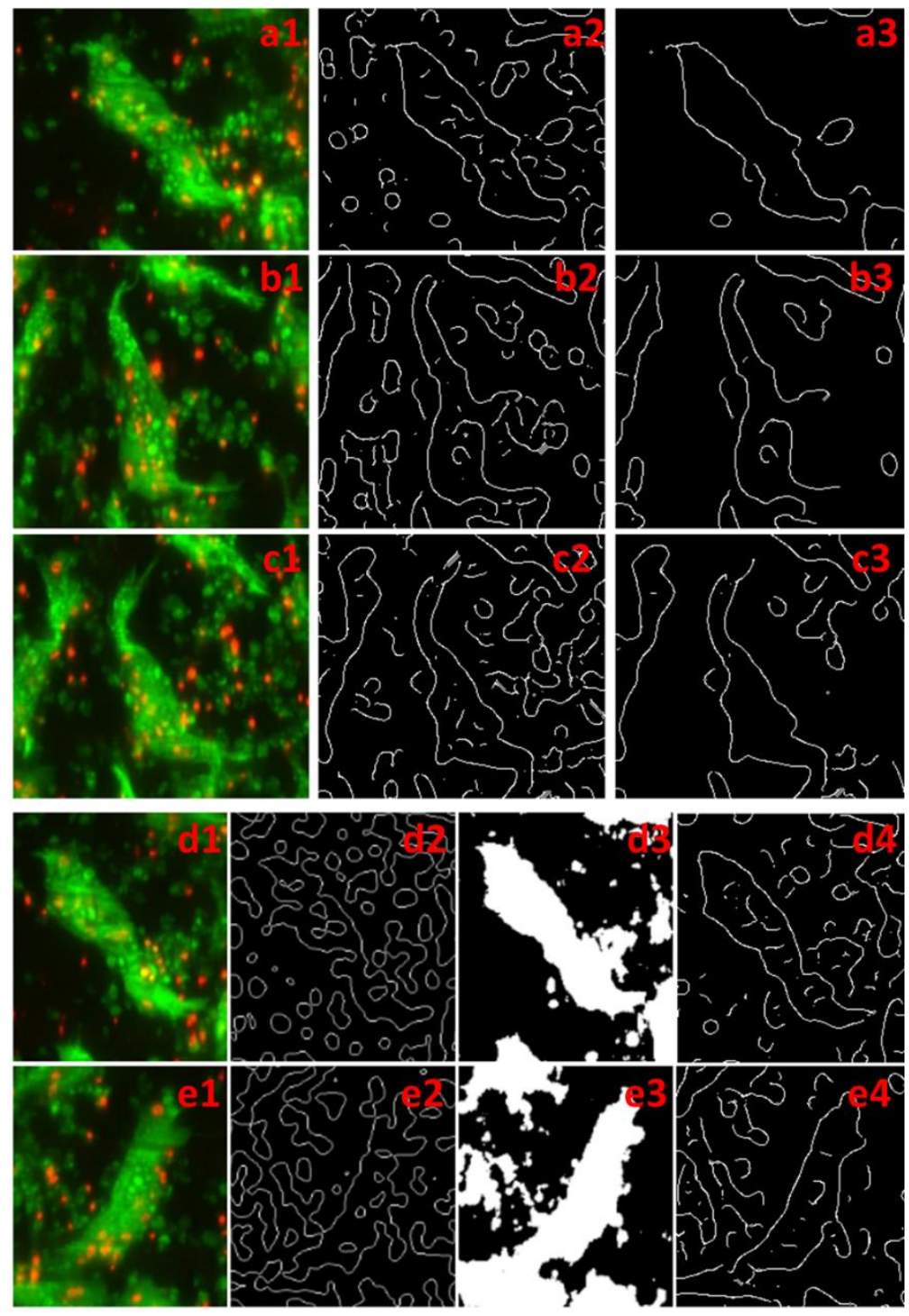

Figure 17. Comparison of different edge detection techniques.

a2, b2, c2, d4, e4: Output of canny edge detection. a3, b3, c3: Output of kmeans clustering for muscle boundary extraction. d2, e2: Output of laplacian edge detection. d3, e3: Output of isodata thresholding technique. 


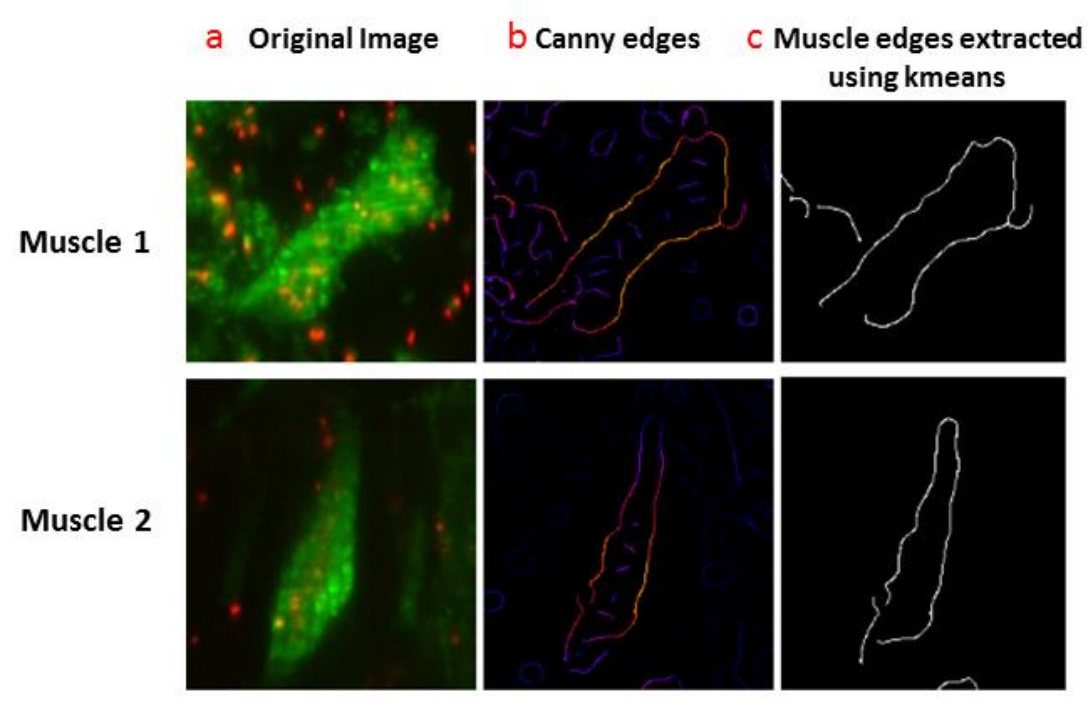

Figure 18. Muscle boundary extraction using canny detector.

(a) Input image of muscle cell. (b) Edges obtained by canny edge detector. The color indicates the value of Gaussian derivative (yellow indicates high value, blue indicates low value). (c) Muscle edges extracted after applying kmeans to average value of Gaussian derivative of the edges.

\subsection{Watershed algorithm}

Watershed transform performs region based segmentation of images. It treats an image like a topological map where intensity of the pixel represents its height. Watershed transform extracts catchment basins surrounded by ridges and the line on the ridge are considered the edges of the image.

I used a marker based morphological watershed segmentation algorithm [104][106]. A morphological gradient of the image is calculated using a disk structuring element. Then, an extended minima transform is applied to the image to find the regional minima. This controls the number of minima by removing any trough with a depth below the user defined threshold $h$ (H-minima transform). Finally, these minima points are used as markers to apply the watershed segmentation. Therefore, the total number of regions produced by the watershed segmentation can be regulated by the number of markers. When the value of $h$ is high, regions that have strong edges are obtained, and when $h$ is low, regions with weak edges are obtained. In Figure 19, the watershed regions for three different values of $h$ are shown. There should be an optimal number of regions to maintain the balance between execution time and degree of accuracy. 


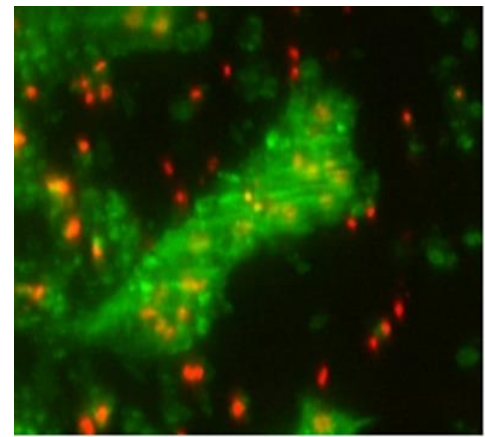

Original Image

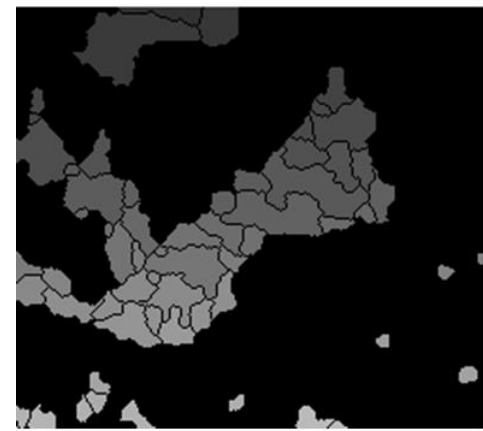

$h=7$
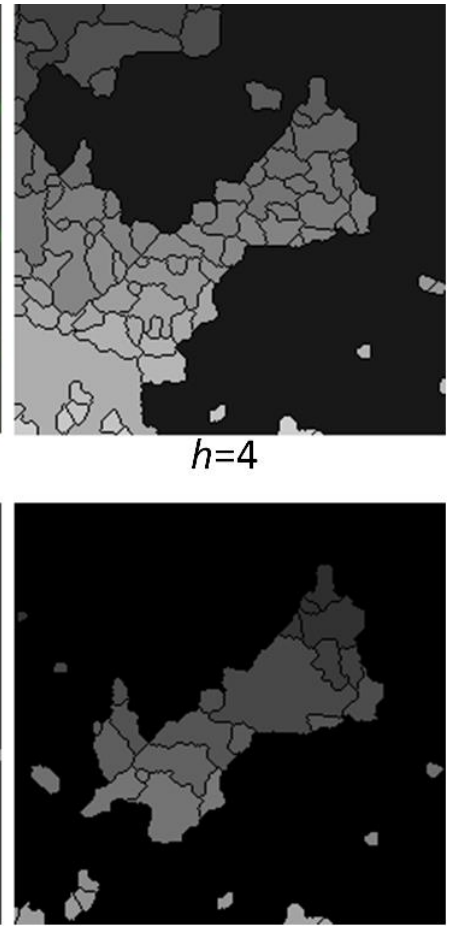

$h=10$

Figure 19. Watershed regions generated at different values of threshold $h$.

Another technique which is often used to generate over-segmentation are graph based. I compared the performance of watershed over-segmentation with $\mathrm{N}$-cut based superpixel generation [68]. Graph cut segmentation designs a graph where pixel value are the nodes and edges represent affinity between pixel pairs. The graph is partitioned such that a global cost function is minimized. Normalized cut or N-cut uses a spectral approximation method whose run time complexity is dependent on number of pixels. Also, due to a compactness constraint, $\mathrm{N}$-cut generates quasi-uniform regions. Its performance on muscle images is shown in figure 20 [107]. Since the smaller region generation is based on size of region instead of intensity, in many cases they miss smaller structures in the muscle image. Example of the issue discussed above is shown in figure 20 a2-3, b2-3 (yellow arrowhead), where the edge at the tip of muscle cell (a2) and the boundary between debris and muscle is missed by N-cut. Since, the boundaries generated by watershed adhere to the intensity homogeneity constraint; there performance is better in muscle images (Figure 20 b1-2). Also, morphological watershed segmentation is much faster than $\mathrm{N}$-cut segmentation 

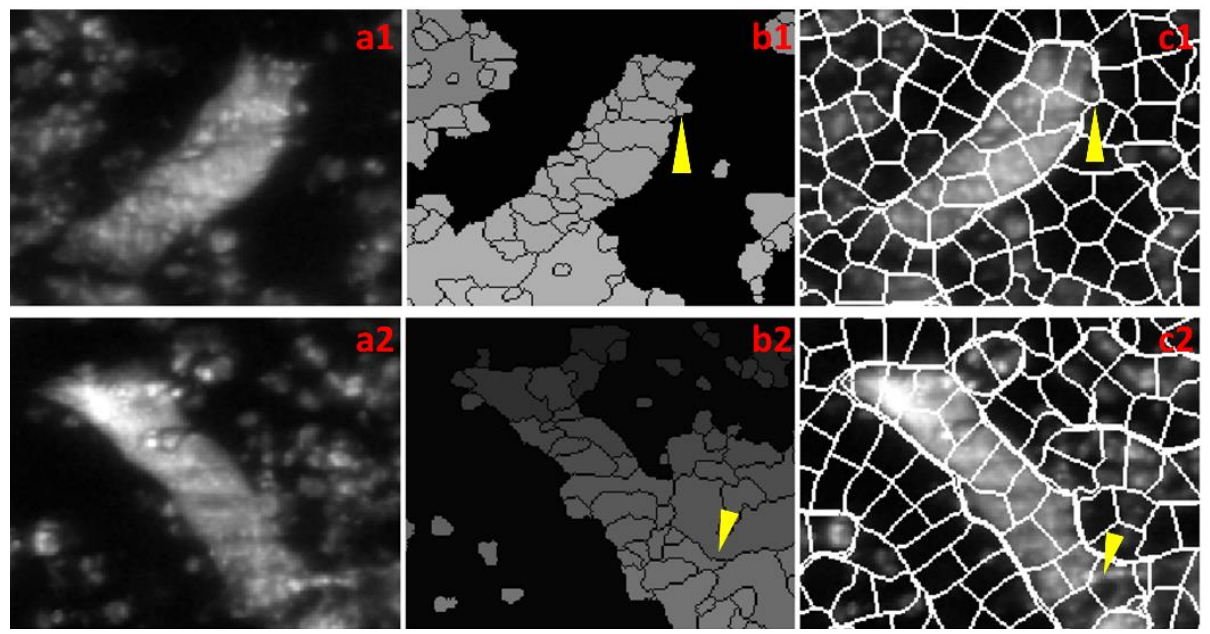

Figure 20. Comparison between the performances of morphological watershed (b1, b2) and N-cut (c1, c2) segmentation on muscle image. Yellow arrowhead shows the edge which N-cut was not able to identify.

Therefore, the topological advantage i.e. closed contours of watershed technique can be combined with high localization accuracy of canny edge detector to extract the missing muscle boundaries. To achieve that, there is still a major issue that is to be solved. How do one identify the actual muscle boundary among multiple edges generated by watershed? The edges that were extracted using canny detector can be used to extract high confidence watershed edges based on proximity. However, what about the regions (e.g. muscle and debris in close proximity, two muscles lying very close to each other) where canny could not detect edges. Debris lying close to muscle cell is most common problem in our dataset. At many time points they are difficult to identify based on intensity and texture because the muscle cells also have similar profile. For such cases, structural information about the shape of muscle cell is required. Since I am working on time series images, the shape information can be extracted from the previous time point. In the next section, I'll discuss the techniques used for comparing two shapes. 


\subsection{Chamfer Distance}

There are multiple techniques available for shape matching. Hausdorff distance is equivalent to the largest value among the shortest distances between two set of points (two shapes). However, it is highly sensitive to outliers and does not give a cumulative shape differences. Shape context have also been used for matching shapes. In shape context based matching: first, points are sampled on the shape boundary; second, a log-polar histogram is generated using the sampled points and last, the histograms of two shapes are compared to identify how similar they are. Due to the nature of shape context design, it is cannot differentiate very small changes in shapes like a protrusion on muscle cell which lies within same bin of log-polar histogram. Another method called chamfer distance [108] has been used extensively for shape-based template matching for object detection. It is used to measure the similarity between two shapes. Let $U=\left\{u_{i}\right\} i=1 \ldots n$ and $V=\left\{v_{j}\right\} j=1 \ldots m$ be the contour points of two shapes; the chamfer distance is the average distance between each point $\mathrm{u}_{\mathrm{i}}$ and its closest point in $\mathrm{V}$ :

$$
C(U, V)=\frac{1}{n} \sum_{u_{u_{i} \in U}} \min _{v_{j} \in V}\left|u_{i^{-}} v_{j}\right|
$$

The chamfer distance can be calculated using the distance transform. One of the shapes is taken as a binary template, whereas distance transform is calculated for the other shape. The chamfer distance is given by the mean of the distance transform values at the boundary of the binary template. In order to increase the robustness of chamfer distance against noisy or cluttered edges, a threshold distance is used [109]:

$$
\left.C(U, V)=\frac{1}{n} \sum_{u_{i} \in U} \underset{v_{j} \in V}{\max (\min }\left|u_{i}-v_{j}\right|, \tau\right)
$$

where $\tau$ is the threshold value. Further improvement in the robustness of chamfer distance has been obtained using edge orientation information [110] (Figure 21). The 
orientation mismatch is given by the mean difference in orientation between a point on the template $U$ and its closest point on the edge map $V$.

$$
O(U, V)=\frac{1}{\pi n} \sum_{u_{i} \in U}\left|\alpha_{u_{i}}-\alpha_{P(V)}\right|
$$

$\alpha_{u_{i}}$ is the angle at point $u_{i}$ in $U$, and $\alpha_{P(V)}$ is the angle at a point $P$ in $V$ closest to $u_{i} . \mid \alpha 1$ $\alpha 2 \mid$ gives the smallest difference between $\alpha 1$ and $\alpha 2$. The oriented chamfer distance is determined by:

$$
\text { Docd }=(\sigma-1)^{*} C(U, V)+\sigma^{*} O(U, V)
$$

where $\sigma$ is the orientation specificity parameter.
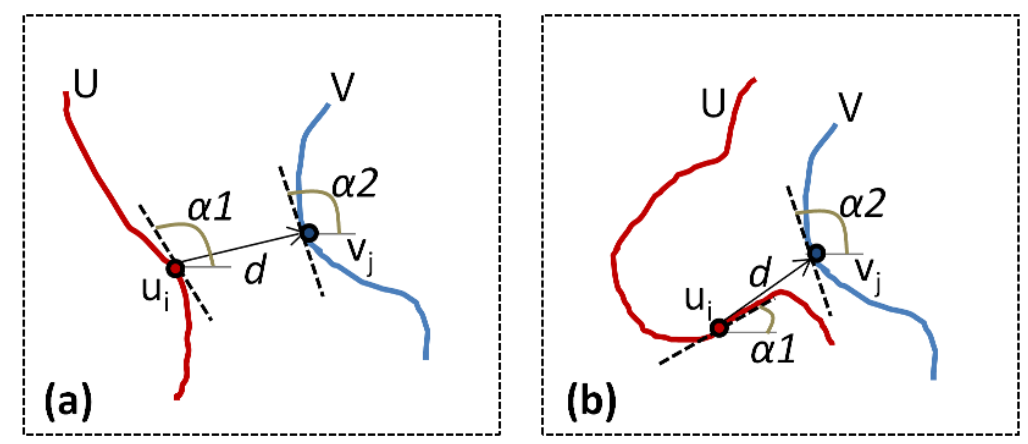

Figure 21. Oriented chamfer distance.

a) The OCD at point $u_{i}$ consists of two components. The distance of $u_{i}$ with the closest point on $V$ i.e. $v_{j}$ and the smallest difference between the orientation of contour at these points $|\alpha 1-\alpha 2|$. b) Since the shapes of contours $U$ and $V$ are different, the orientation difference between $u_{i}$ and its closest point $v_{j}$ is higher than (a).

Previous methods have also used edge information to weight the distance transform, such that more significance is given to strong edges than to weak edges [111], [112]; this helps to reduce the impact of weak edges during shape matching.

Due to abrupt changes in muscle shape (debris attachment to muscle), the capability of oriented chamfer distance to measure the similarity between shapes based on distance and orientation differences at each set of points on two shapes is better than hausdorff or shape context where distance is measure only at a single point or sampled 
points. Therefore, in my segmentation method, the chamfer distance is used to the measure the similarity between muscle boundaries of two subsequent time points. High similarity indicates a reduced chance of muscle boundary lying inside the debris. However, chamfer distance alone cannot provide robust results. I have designed a methodology that combines chamfer distance with edge confidence map for muscle segmentation.

\subsection{Methodology}

Based on discussions in previous sections, a new muscle segmentation algorithm that follows a two-step approach was proposed: First, a watershed algorithm is implemented to divide images into multiple regions while preserving edges and a confidence map is generated for the edges of watershed regions using canny edge detection. Secondly, these 'watershed' regions are classified into muscle and nonmuscle classes based on confidence map and shape comparison. In this work the second step is referred to as a region classification algorithm. In order to avoid incorrect classification of non-muscle regions near actual muscle boundary the shape information derived from muscle boundary at a previous time point is used. I'll discuss the methodology of muscle segmentation in detail next. Figure 22 shows the schematic diagram of the muscle segmentation methodology: 


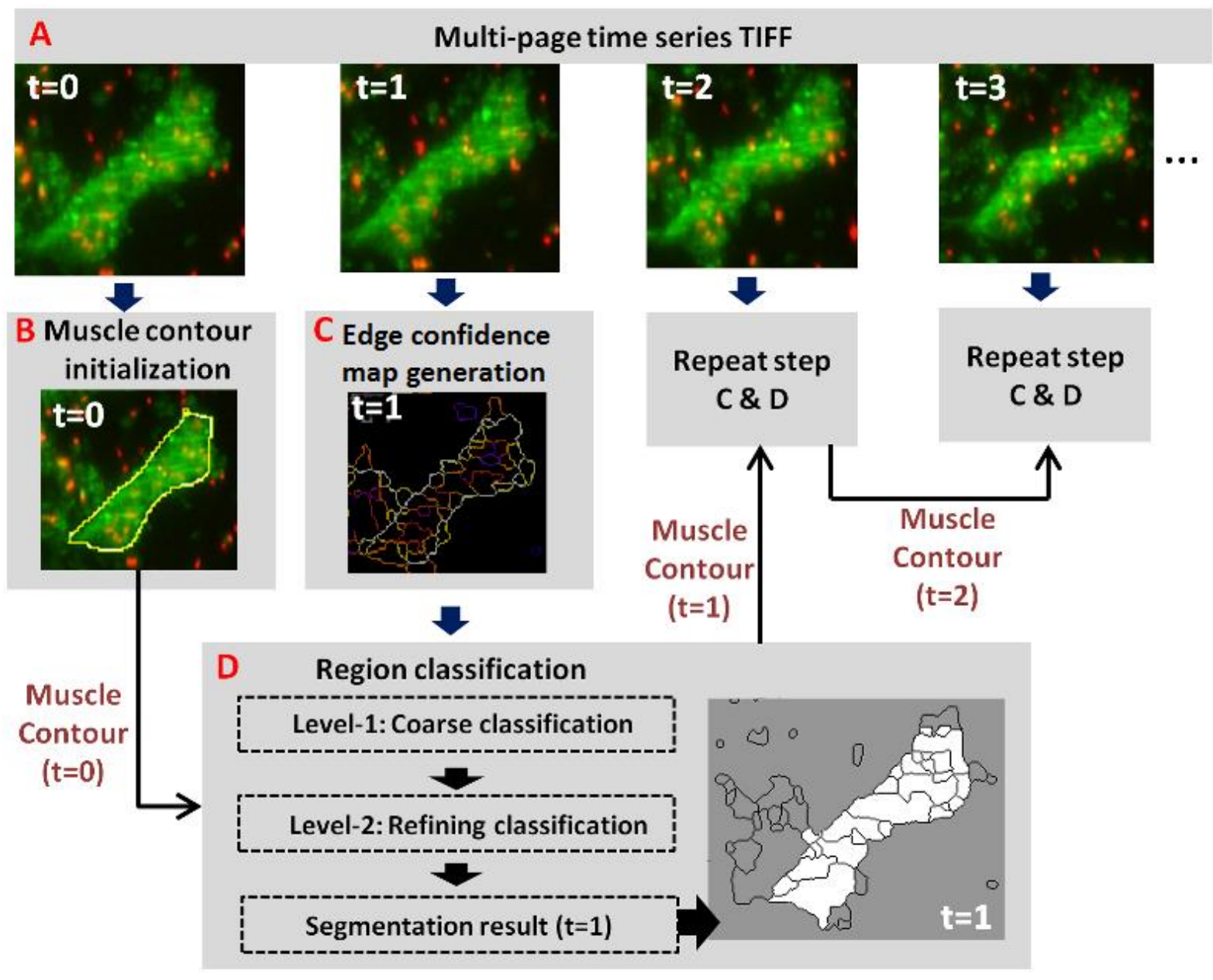

Figure 22. Schematic overview of muscle segmentation.

A) First four time points of a time series stack. B) Muscle boundary at $t=0$ shown in yellow. C) Edge confidence map generated at $\mathrm{t}=1$ using watershed segmentation and canny edge detector. D) Classification of watershed regions at $\mathrm{t}=1$. The white watershed regions constitute the segmented muscle at $\mathrm{t}=1$. The muscle contour of the previous time point $(\mathrm{B})$ is required as an input for region classification.

\subsubsection{Initializing muscle boundary}

During the region classification step, shape information from the boundary of the muscle cells at a previous time point is used. Therefore, it is crucial that the boundary of the muscle cells at the time point $t=0$ is as accurate as possible. Users can obtain the muscle boundary at $\mathrm{t}=0$ by manually drawing a contour around the muscle or using the level set segmentation method. Figure 22B shows a muscle boundary generated manually at $\mathrm{t}=0$. 


\subsubsection{Edge confidence map generation using watershed segmentation}

As discussed before, watershed segmentation is type of region based segmentation which divides the image into homogenous regions. However, due to noise or texture of the image; it suffers from over-segmentation. Various solutions have been suggested to solve this issue for example, marker controlled and hierarchical watershed [113]. On the other hand, canny operator is a very powerful edge based segmentation technique. However, the edges detected by canny method are not connected. Therefore, post processing is required to connect these edges. In my segmentation method, these two types of segmentation techniques are combined to overcome the problems discussed above and generate a confidence map of edges. The confidence level of an edge is an indicator of the probability that the edge does not belong to the boundary of a muscle cell. After obtaining the muscle boundary from $t=0$, a confidence map is generated for every image from subsequent time points $(\mathrm{t}>0)$ (Figure 22-C). The steps of watershed edge confidence map generation are explained below.

The confidence map is generated on the basis of the distance between the edges obtained by watershed segmentation and canny edge detection technique. A distance transform is applied on the binary image which contains the muscle edges isolated using canny edge detector. The confidence value of a watershed edge $C w$ is given as,

$$
C w=\frac{1}{n w} \sum_{i=1}^{i=n w} C D_{i}
$$

where, $n w$ is the length of watershed edge in pixels, $i$ is the location of the watershed edge pixel and $C D_{i}$ is the value of distance map obtained from canny edges at $i$ location. High value of $C w$ indicate higher chances that the edge does not belong to the muscle. Similarly, low value of $C w$ indicate lower chances that the edge does not belong to the muscle. After calculating confidence value for every watershed edges, the confidence map was obtained (Figure 23). These edge confidence values are used as a feature for region classification algorithm. 
Input Image

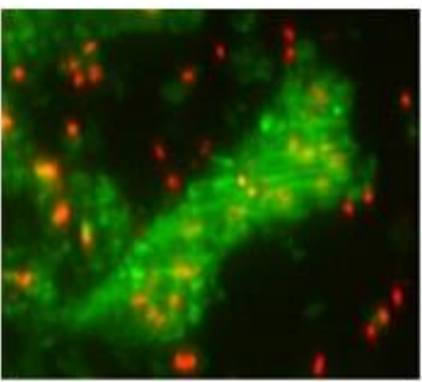

Watershed segmentation

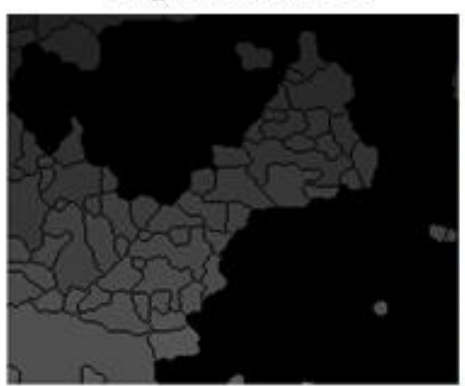

Edge confidence map

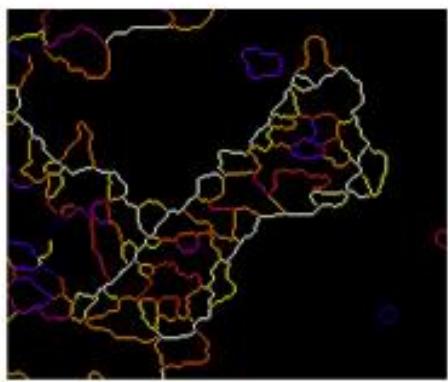

Figure 23. The figure shows the results of watershed segmentation and the corresponding edge confidence map.

The white and yellow edges have low confidence value i.e. they have higher probability of being a muscle boundary.

\subsubsection{Region classification algorithm}

Muscle segmentation requires classification of the watershed regions into muscle and non-muscle regions. I propose a two-level approach for region classification (Figure 22-D). At level 1, a coarse classification is applied and at level 2, the results of level 1 are refined on the basis of a dissimilarity function that is derived from chamfer distance and edge confidence map.

Level 1: The purpose of coarse classification is to reduce the number of regions to be classified based on shape and edge based features. It will save computation time as well as improve accuracy by identifying the segment where muscle is located. The watershed regions are coarsely classified on the basis of their pixel overlap with the muscle segmentation result $M_{t-1}$ from the previous time point. The following criterion is used:

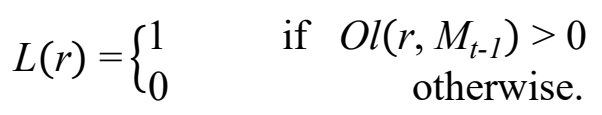

$L$ is the label of the watershed region, $r$ is 0 for non-muscle and 1 for muscle, and $O l$ is the pixel overlap of region $\mathrm{r}$ with the previous time point muscle segmentation, $M_{t \text { - }}$ 
1. After classifying the regions, two segments are obtained: $S_{0}$ containing regions with no overlap $(L(r)=0)$ that correspond to the non-muscle class (shown in grey in Figure 24-A1) and $S_{1}$ containing regions with overlap $(L(r)=1)$ that correspond to the muscle class (shown in white in Figure 24-A1). The label $r_{1}$ in Figure 24-A1 was classified incorrectly. The red contour is the muscle boundary from the previous time point.

Level 2: The results from level 1 are refined by reclassifying the boundary regions of the two segments, $S_{1}$ and $S_{0}$, on the basis of a dissimilarity measure. Each boundary region $r_{b}$ can either stay in its current segment or move to another segment, depending on which option has the smallest dissimilarity measure. For each region $\left(r_{b}\right)$ two dissimilarity measures are calculated: $D_{0}$ with segment $S_{0}$ and $D_{1}$ with segment $S_{1}$. A dissimilarity measure has two components. The first is the confidence value of the edges which was calculated in the previous section.

$$
\begin{aligned}
& I_{0}=\text { Confidence value of edge between } r_{b} \text { and segment } S_{0} \\
& I_{1}=\text { Confidence value of edge between } r_{b} \text { and segment } S_{1}
\end{aligned}
$$

The second component is derived from the difference in the shape of the $S_{1}$ segment (muscle class) from the muscle boundary of a previous time point $B_{t-1} . B_{t-1}$ was compared with two shapes: $S_{1}$ when $L\left(r_{b}\right)=1$ and $S_{1}$ when $L\left(r_{b}\right)=0$. A high shape difference when $L\left(r_{b}\right)=1$ would indicate a high chance of $r_{b}$ belonging to non-muscle class and a low shape difference would indicate a low chance. An example of shape comparison is shown in Figures 24-B4 and 24-B5.

The difference in shape is quantified by measuring the oriented chamfer distance, given by $E_{0}$ and $E_{1}$ for the two shapes [110]. As explained in the previous section, the oriented chamfer distance measures the similarity between two shapes or contours. In this analysis, $U$ is the set of contour points of the muscle cell at a previous time point $B_{t-1}$, and $V$ is the set of contour points of segment $S_{1}$. Therefore, from equation 4 , the oriented chamfer distance is determined by:

$$
E=\frac{1}{2}(\tau(C(U, V)+C(V, U))+(O(U, V)+O(V, U))) .
$$


The weight of the chamfer distance component is given by $\tau$. The overall dissimilarity between region $r_{b}$ and segment $S_{0}$ and $S_{l}$ is given as:

$$
\begin{aligned}
& D_{0}=E_{0} * I_{0} \\
& D_{1}=E_{1} * I_{1} .
\end{aligned}
$$

$E_{0}$ is the oriented chamfer distance between $B_{t-1}$ and segment $S_{l}$ without the region $r_{b}$. $E_{l}$ is the oriented chamfer distance between $B_{t-1}$ and segment $S_{l}$ with region $r_{b}$. The weight of the intensity component is given by $\lambda$. The following criterion for reclassification of region $r_{b}$ was used:

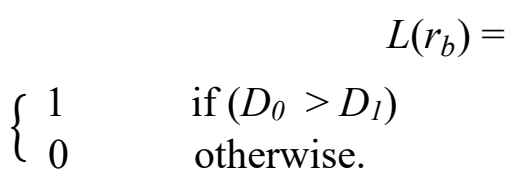

The boundary regions which are farthest from the muscle boundary of previous time point are assessed first for reclassification. If the label of a region $\left(r_{b}\right)$ changes, then the list of boundary regions of the segments is updated. After iterating through all of the boundary regions, the final muscle segmentation is defined by the boundary of segment $S_{1}$. The outcome of classification refinement is shown in Figure 24-B3.

Stopping Criterion: The watershed-based division of image and region classification is repeated for each time point until the end of the time series stack (Figures 22C \& 22D). Whereas, the chamfer distance, $C(U, V)$, between the muscle contours of adjacent time points is used as a criterion to stop the segmentation of a muscle. If the difference between the shapes of muscle boundaries is greater than a predefined chamfer distance threshold $(\gamma)$, then the segmentation stops; indicating that segmentation is not performing well. If the criterion is not met at any time point, then the segmentation continues until the end of the time series stack. 


\section{Region classification}

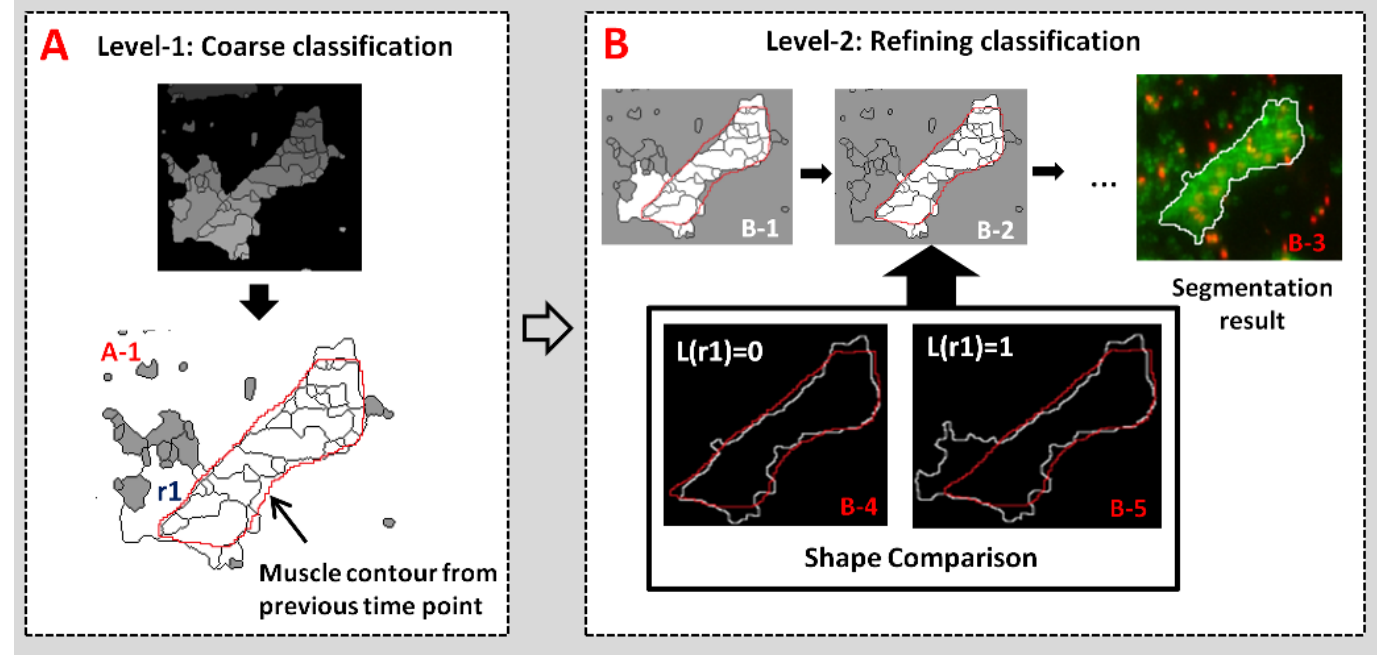

Figure 24. Region classification and its subsections.

A) Coarse classification of watershed regions at $\mathrm{t}=1$ based on their overlap with muscle boundary ( in red) at $\mathrm{t}=0$ (A1). B) Refining classification. The intermediate results of refining step (B1-B2). The final result of refining step is shown in B3. An example of shape comparison for boundary region $r l$ is shown in B4 \& B5. The contour in red is the muscle boundary of the previous time point $B_{t=0}$ and the grey contours show the boundary of a segment formed by watershed regions, classified as muscle (SI) when $L(r l)=0(\mathrm{~B} 4)$ and $L(r l)=1$ (B5). The difference between shape of $B_{t=0}$ and $S 1$ is higher when $r l$ belongs to muscle class (i.e. $L(r l)=1$ ).

\subsection{Muscle Morphological Features}

FMAj calculates various morphological features like average diameter, area, length, elongation and extent for the segmented muscle boundaries. Elongation and extent quantify the shape of the muscle cells. Elongation, as the name suggests, quantifies the elongation of muscle cells and is defined by the ratio of length of minor and major axis. Extent, on the other hand, measures whether the muscle cells are rectangular in shape. The value of extent is affected by change in muscle orientation and muscle bending. The extent feature was modified to quantify the muscle shape along the medial axis of muscle cell. 


\subsubsection{Extent-S}

Extent is the ratio of the area of muscle cell and the area of its bounding box (Figure 25). To measure the rectangular-ness of muscles irrespective of whether they are bent or not, the bounding box of muscle cell along its medial axis needs to be extracted.

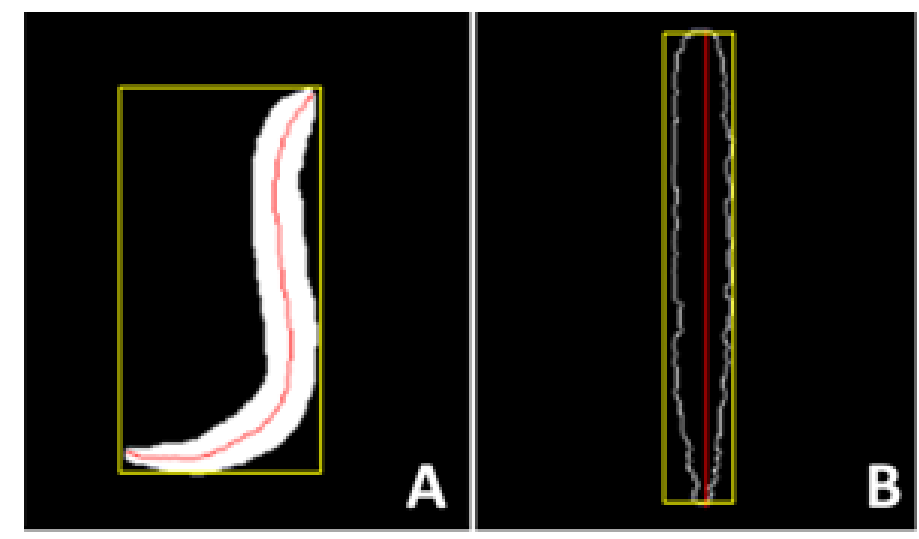

Figure 25. (A) Bounding box of a muscle cell. (B) Bounding box of (A) muscle cell if it was not bent.

The extent (Extent-S) for a straight muscle cell, in other words along the medial axis is calculated by,

$$
\text { Extent-S }=A /\left(L^{*} W\right)
$$

Where, $A$ is the area of muscle cell, $L$ is the length of muscle's medial axis and $W$ is the largest width of muscle cell along its medial axis. The figure 26 shows the muscle cell contours and the corresponding values of extent and extent-S. In figure 26 , the value of extent is low because the muscle 1 is oriented at a certain angle. The bent structure of muscle 2 in figure 26 results in low value of extent. 


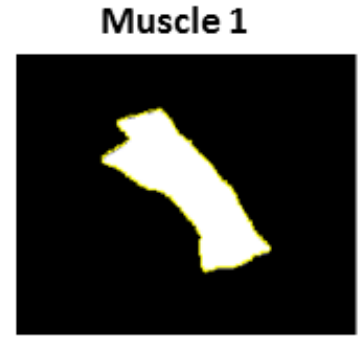

Extent $=0.395$

Extent- $\mathrm{S}=0.664$
Muscle 2

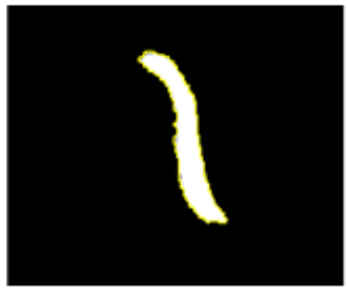

Extent $\mathbf{~} \mathbf{0 . 2 8 4}$

Extent- $\mathrm{S}=0.868$

Figure 26. Comparison between the values of extent and extent-S in two different muscle cells.

\subsubsection{Polar histogram based morphological feature}

Just like extent, other features are also affected by the bending of muscle cell for example elongation. Although, bending of muscle cell is in itself a biological phenotype; however it is difficult to describe the shape of muscle cell along the medial axis. For this reason, first the muscle cell is straightened along its medial axis and then its contour is converted into histogram to generate different types of shape features. The medial axis acts as a spatial reference to straighten the muscle cell (Figure 25). The steps used for straightening are explained below:

1. Find the skeleton of the muscle cell.

2. For a point $\mathbf{P}$ on the muscle boundary, find its closest point on the skeleton $S_{\mathrm{p}}(\mathrm{x}$, y). The distance between these points is given by $d x$.

3. Find the length of medial axis $d y$ till point $\mathrm{P}(\mathrm{x}, \mathrm{y})$.

4. If one assumes that the straightened medial axis lies at $x=0$, then the new coordinate of the point $\mathrm{P}$ of the object is given by $( \pm d x, d y)$. The sign of $d x$ depends on whether point $\mathrm{P}$ lies on right or left of the skeleton.

5. Repeat the steps from 1-4 for every point on the muscle boundary.

Next, I create a polar histogram of the muscle cell contours. The polar histogram is the distribution of the points on the contour grouped according to their angle and distance from a reference point. The midpoint of the medial axis of muscle was used as the reference point. A fixed number of randomly chosen points are used from the muscle contour to generate histogram. After grouping the points, the histogram $H(A \times D)$ was calculated; where $A$ is the number of bins for angle and $D$ is the number of bins for 
distance. Figure $27 \mathrm{~b}$ shows a muscle cell with overlapping polar bins. The point from muscle contour will be grouped according to which block it falls into.

(a)

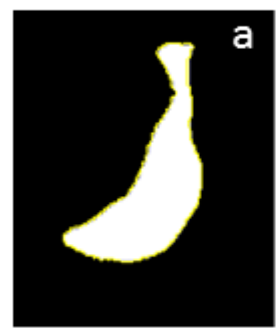

$E=0.78$

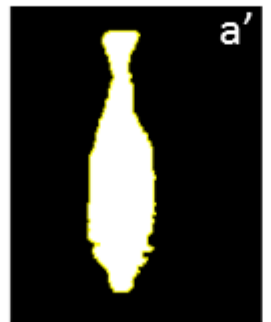

$\mathrm{E}-\mathrm{S}=\mathbf{0 . 9 2}$ (b)

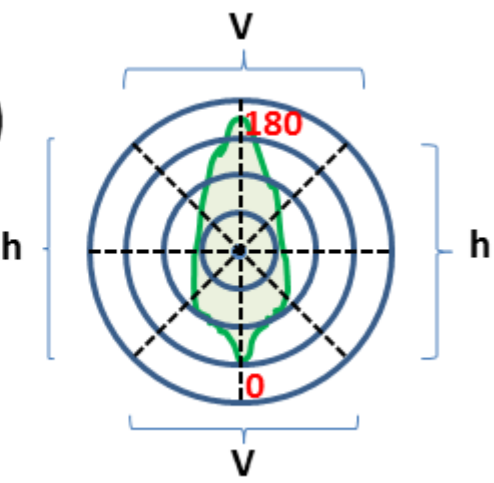

Figure 27. Polar histogram based derivation of elongation feature.

(a) The figure shows muscle cell with the value of elongation (ellipse fitting) at the bottom. (a') The figure shows the straightened muscle cell (a) and the value of elongation-S (E-S) at the bottom. (b) The polar bins overlapping with muscle cell and its center positioned at the middle of muscle's medial axis. $\mathrm{v}$ and $\mathrm{h}$ show the vertical and horizontal group of bins.

I designed a new feature to evaluate the elongation of muscle cell. The angle bins were grouped into two categories: vertical and horizontal as shown in figure $27 \mathrm{~b}$. The elongation of straightened muscle cell or 'elongation-S' is defines as,

$$
\begin{aligned}
\text { elongation- } \mathrm{S} & =\mathrm{V}_{\mathrm{s}} /\left(\mathrm{V}_{\mathrm{s}}+\mathrm{H}_{\mathrm{s}}\right) \\
\mathrm{V}_{\mathrm{s}} & =\sum(H(A v, d) * l) \\
\mathrm{H}_{\mathrm{s}} & =\sum(H(A h, d) * l)
\end{aligned}
$$

where, $A v$ is the subset of angles that fall in the vertical group, $A h$ is the subset of angles that fall in the horizontal group and $l$ is the upper distance of the $d_{t h}$ distance bin. High value of elongation-S indicates that the muscle cell is much longer and less wide. An example comparing the elongation calculated using ellipse fitting and elongation calculated using my method is shown in the figure 27a. Due to bending of the muscle cell the value of elongation (Figure 27a) is lower than the elongation-S (Figure 27a'). 


\subsection{Summary}

The major contribution of this chapter is the new muscle segmentation algorithm. The objective is to segment large number of time series images with least amount of manual intervention. In order to achieve that I combined watershed segmentation with a dissimilarity based region classification technique. The advantage of the region classification method is two folds: first it uses an edge based confidence map to accurately segment low intensity regions of muscle cell and second, a chamfer distance based shape metric to reduce the false classification of non-muscle regions as muscle. Due to the shape based constraint, the user need to initialize muscle boundary only once at $\mathrm{t}=0$ and the subsequent time points will be segmented automatically. The performance of muscle segmentation algorithm is discussed in chapter 6. 


\section{Chapter 5 Nuclear Spatial Pattern}

\section{Analysis}

Previously, we found that the nuclei in abdominal dorsal internal oblique muscles (DIOM)/persistent muscles of Drosophila show changes in myonuclear distribution during metamorphosis [114]. The nuclei form a two-row formation during prepupa stage (Figure 28a). After head eversion, the nuclei inside persistent muscles retain their two-row formation whereas the nuclei inside doomed muscle become part of the fragments of dead muscles (sarcolytes). Hereafter, I will call the nuclei inside the persistent muscles as internal nuclei and nuclei inside sarcolytes as external nuclei. During early pupal development, the nuclei inside persistent muscle start migrating towards the medial axis of muscle into a one-row formation. Around the mid-pupal stage, we observed anti-polar migration of nuclei and their localization in the muscle center. After this stage, the nuclei migrate back to the poles and formed a one-row structure. Although the muscles increase in size towards the end of metamorphosis, the nuclei stay in one-row formation. Furthermore we demonstrated that knockout of Cpl and several autophagy-related genes affect myonuclear distribution (Figure 28c) [114]. To better understand the effects of genetic perturbations on myonuclear distribution, a quantitative analysis of nuclear migration and localization is required. The advantage of quantitative analysis of myonuclear distribution is two folds. First, quantitative analysis helps in identifying new nuclear phenotypes which might not be noticed visually. It can also be used to compare the phenotypic effects of different genetic perturbations on myonuclear distribution using statistical tests. Second, semiautomated/automated quantitative tools enable us to study large number of time series images with least amount of manual interference.

Nuclear spatial pattern analysis has been used previously to investigate the distribution of sub-nuclear structures such as centromeres [115], nuclei in multinucleated muscles [33], and nuclei in Drosophila embryos [116]. In recent years, many studies in myonuclear distribution has been done. However, each of these studies were done on very few genes and they did not analyze nuclear distribution for long durations 
( 4-5 days). Previously, it was found that lack of plectin isoform 1 (P1) affected the orientation as well as spacing between nuclei in mice muscle fibers [117]. Manual tracking imageJ tool was used to study mobility of nuclei [118]. In another research which found that nesprin $1 \alpha 2$ plays a critical role in nuclear distribution in mice skeletal muscle; nuclear distance measurements were done manually [119]. Manual selection of nuclear centroids was done in an analysis on the involvement of microtubule motor proteins on nuclear movement during skeletal muscle differentiation [120]. A study which found that overexpression of PGC-1 $\alpha$ results in reduction in internuclear distances used custom MATLAB tool for analysis [121]. Whereas, a study on regulation of nuclear positioning by nesprin dependent recruitment of kinesin-1 performed nuclear distribution analysis using Volocity 3D Image Analysis tool [122]. They used fixed cell images for analysis of nuclear distribution i.e. nuclear pattern over a period of time was not studied. All of the studies mentioned above used inter-nuclei distances or count of nuclei in muscle fibers to characterize the nuclear spatial pattern. The major differences between previous studies and my methodology are as follows:

1. This is the first study to analyze changes in myonuclear distributions during the complete metamorphosis stage in Drosophila (approx. 3-4 days).

2. Nuclear spatial pattern analysis pipeline which I designed is fully automated i.e. user does not have to intervene between nuclear segmentation to feature generation.

3. In this dataset, during mid pupal development the nuclei move very close to each other making it difficult to locate individual nucleus. For this reason, I could not use inter nuclear distances to study nuclear distribution which requires nuclear centroids. Therefore, spatial pattern features based on nuclear region were designed.

4. Our dataset poses a special problem i.e. Drosophila abdomen has two types of nuclei i.e. the nuclei inside muscle cells (internal nuclei) and the nuclei present inside debris produced during histolysis of muscle cell (external nuclei). Only internal nuclei are required for nuclear spatial pattern analysis. However, it is difficult to identify the external nuclei which appear to be inside muscle cells 
due to $2 \mathrm{D}$ projection. Therefore, an additional step to classify internal and external nuclei was designed.

5. The myonuclei shows many subtypes of non-random distribution. They show one or two row like distributions along the muscle cell. In addition, the myonuclei show anti-polar and polar movements during different developmental stages of Drosophila. Thus, features which can characterize these patterns and quantify the change over time were designed.

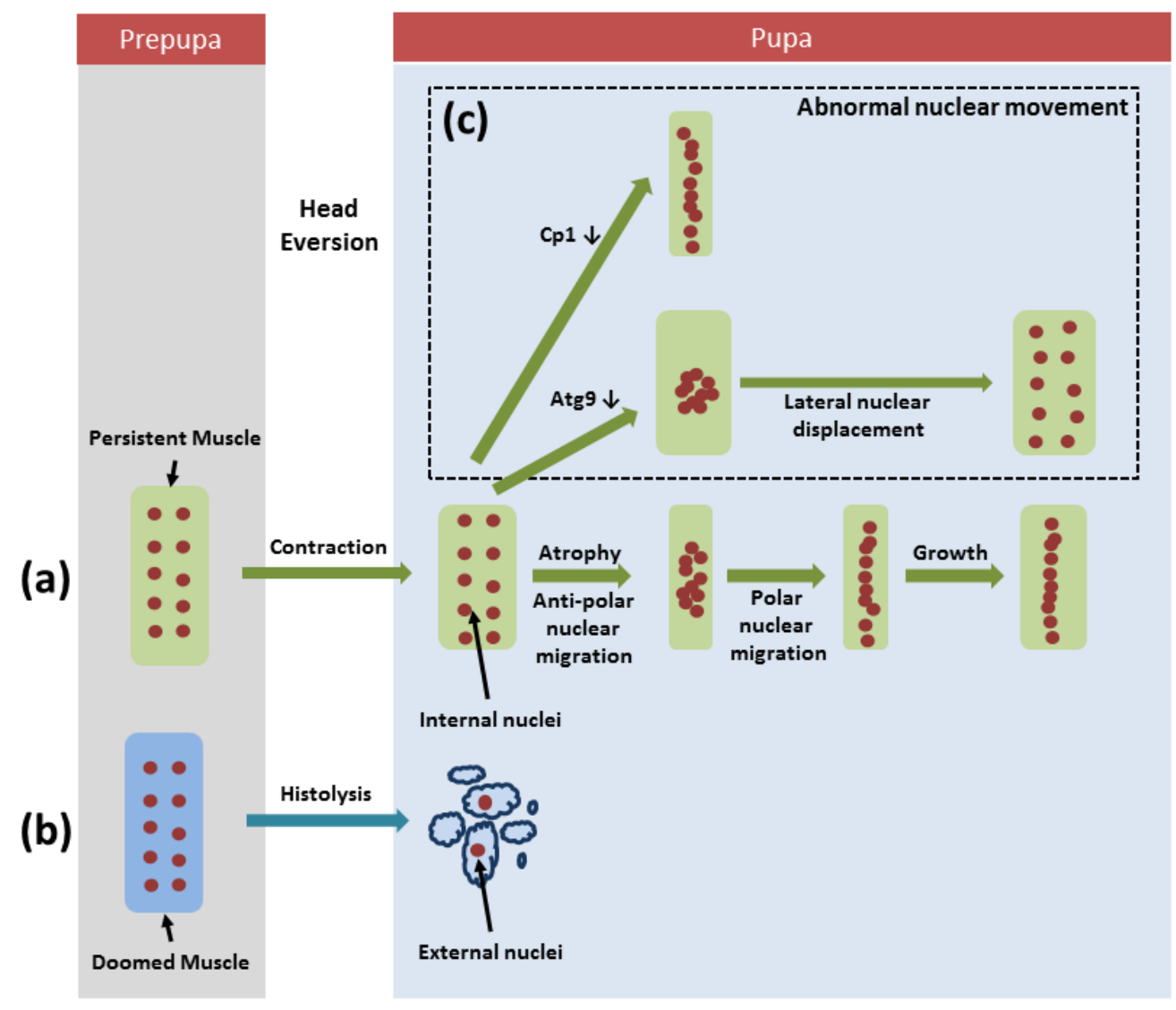

Figure 28 Schematic diagram explaining different stages of nuclear localization.

(a) In persistent muscles, the nuclear positioning transitions from an initial two-row like formation in prepupae to a clustered distribution in mid-pupation, and lastly a one row formation in late pupation. (b) A sub-set of DIOM undergo histolysis and create muscle debris with nuclei inside them. These nuclei form the external nuclei. (c) Knockdown of $C p l$ and Atgs affect the myonuclear distribution. 
Next, I'll will discuss the spatial pattern analysis pipeline to study the effects of genetic perturbations on the distribution of nuclei in remodelled Drosophila muscles during metamorphosis. The pipeline consists of two sections:

1) Extraction of nuclear region inside persistent muscles: I propose an algorithm which is capable of classifying myonuclei into internal and external nuclei. First, I detect myonuclei by performing nuclear segmentation and excluding nuclei outside of the muscle boundary which were obtained using FMAj tool. Next, I track the movements of remaining nuclei and classify them into internal and external nuclei based on their movements. The regions corresponding to the internal nuclei are used to calculate the nuclear spatial pattern features. Performance tests showed that my approach for segmentation and classification of nuclei has very high accuracy in extracting internal nuclei.

2) Nuclear spatial pattern feature generation: I designed new spatial pattern features to quantify the myonuclear distributions like anti-polar/polar migration and one/two row formation of internal nuclei during pupal development. I also demonstrate that these features are capable of quantifying the nuclear distribution patterns in a case study on phenotypic characterization of targeted gene silencing by RNAi of $C p 1$ and autophagy related genes. 


\subsection{Nuclear Spatial Pattern Analysis}

A schematic diagram of the nuclear spatial pattern analysis has been shown in figure

29.

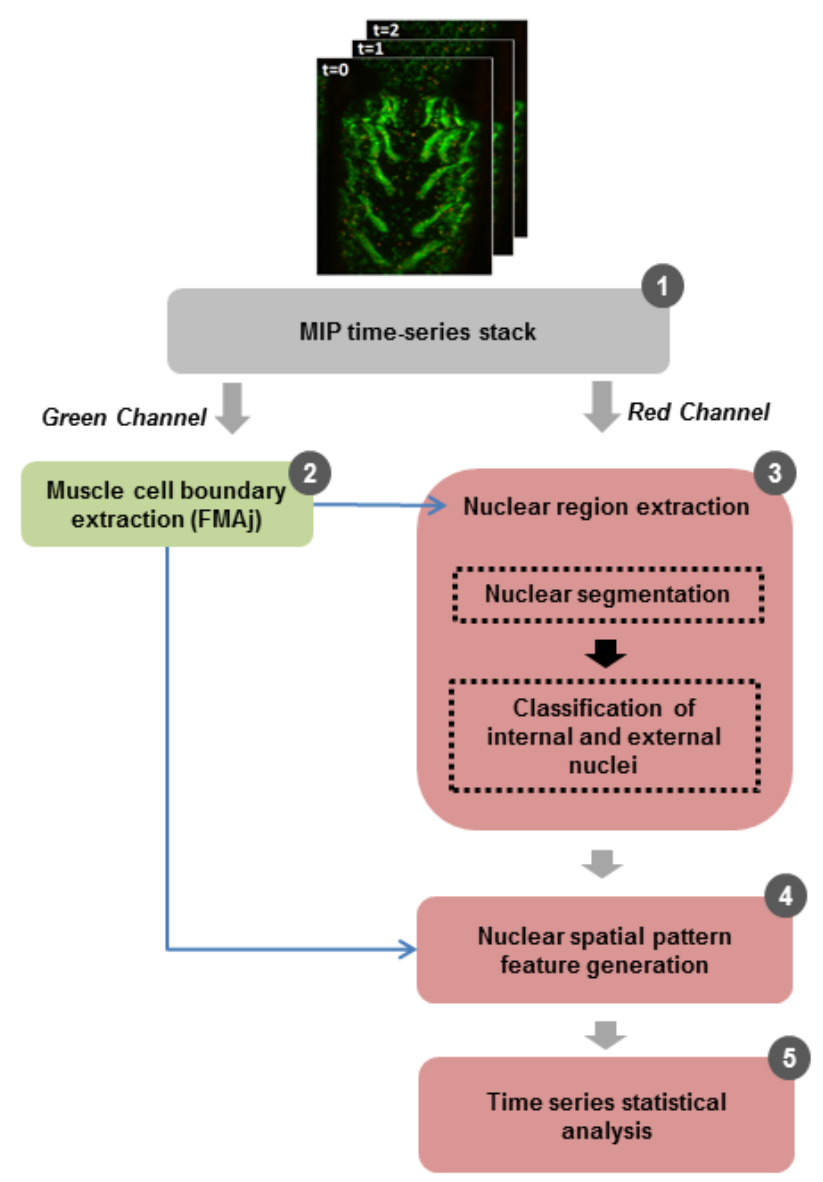

Figure 29 Workflow of the nuclear spatial pattern analysis pipeline.

(1) The pipeline takes a multi-tiff time series stack as input. (2) The muscle cell boundary is extracted using FMAj tool. (3) The colour channel which contains nuclei is segmented to obtain the nuclear regions. The muscle boundary is used to remove nuclei which lie outside the muscle cell. The external nuclei (present inside the fragments of dead muscle) are identified and removed from the segmentation results to avoid incorrect feature calculations. (4) \& (5) Nuclear spatial pattern features are calculated using the extracted nuclear regions and these features are used for time series statistical analysis of the myonuclear distributions and localization during metamorphosis. 

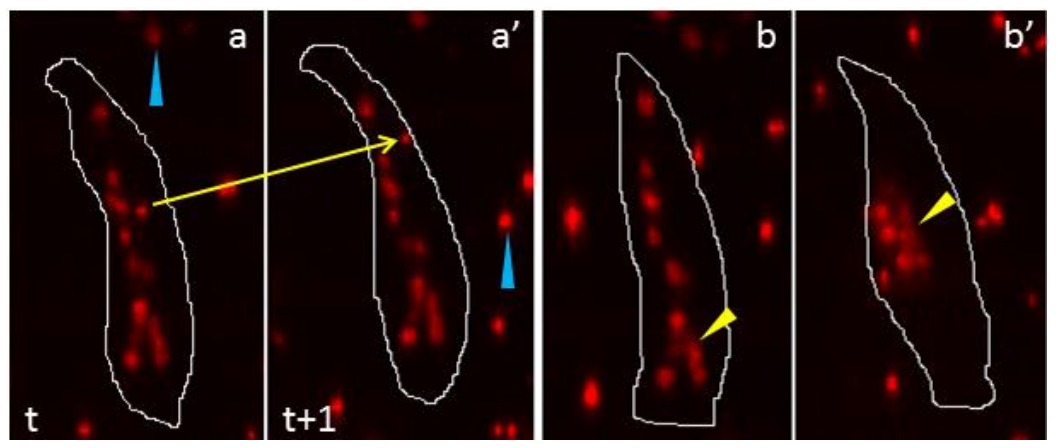

Figure 30 a) Example of internal and external nuclei. b) Examples of nuclei clustering which complicates the tracking of nuclei.

(a) The arrow show the movement of an external nucleus over a muscle cell between time points $t$ (a) and $t+1$ (a'). The arrowhead show an external nucleus located outside muscle cells. (b) \& (b') The arrowheads show the clumping of nuclei in two different muscle cells.

\subsubsection{Nuclear region extraction inside persistent muscles}

As mentioned before, apart from the nuclei inside the persistent muscles (internal nuclei), the nuclei inside dead muscle fragment which were generated during muscle histolysis (external nuclei) are also present in the pupa abdomen, as shown in Figures 30a, a'. For calculation of nuclear features, only the region occupied by internal nuclei are required. Therefore, after nuclear segmentation, the removal of external nuclei from segmented nuclear regions is an important step in myonuclear spatial pattern analysis. The external nuclei which are located outside the persistent muscle can be removed easily using muscle boundary. However, it is difficult to remove external nuclei which appear to be inside persistent muscle due to 2D projection; whereas they are actually moving above the persistent muscles. 3D images could not be used for removal of external nuclei due to low $\mathrm{z}$ resolution. To tackle these problems, I designed a new algorithm for extracting region occupied by internal nuclei. The steps of algorithm are:

\subsubsection{Muscle segmentation}

As mentioned before, the muscle boundaries are required to remove external nuclei located outside persistent muscles. The color channel of input stack containing muscle cells is used for segmentation. The muscle boundaries are obtained by using FMAj. 
Morphological features are extracted from muscle boundary to understand the relationship between nuclear distribution and muscle mass change.

\subsubsection{Nuclear segmentation}

Due to low resolution of images, it is difficult to extract boundaries of nuclei when they are close to each other. For example, a clustered group of nuclei appeared as a large blob of bright fluorescence, as shown in figures 30b, b'. For this reason, instead of detecting each nucleus, regions of nuclei are extracted. Each region can contain one nucleus or multiple nuclei. Nuclear segmentation is used to detect myonuclei. To avoid incorrect segmentation due to imaging noise, images are smoothened using a biexponential edge preserving smoother (BEEPS) [123]. This step smoothens the high intensity spots near the muscle boundary which occur due to dual channel imaging, while retaining the edge information of nuclei.

For segmentation of nuclei, I use the negative Laplacian of Gaussian (LoG) filter based scheme [124]. The LoG filter has been used previously to find dark circular spots of radius $\sigma$ surrounded by bright backgrounds [125], [126]. The general idea is that after Gaussian blurring, the intensity distribution of a nuclei form a smooth ridge and LoG filter can locate the nuclei by detecting the peak point of these ridges. A negative LoG kernel is used (i.e., $-L_{\sigma}$ ) to enhance bright nuclei surrounded by a dark background. Here, $\sigma=6$, the average radius of myonuclei in our dataset, is used. In muscle images, the LoG filter increases the intensity of the regions where nuclei are present. Nuclei are segmented by applying binary thresholding to the filtered image. Any pixel of intensity 255 was labelled as nuclear region whereas the rest of the pixels were treated as background. Figure 31a shows the results of different stages of nuclear segmentation. Smoothening of image results in decrease in the number of false positives during segmentation (Table 5). Also, using LoG filter as pre-processing step, improves the segmentation of nuclei which have relatively low intensity as compared to others. 


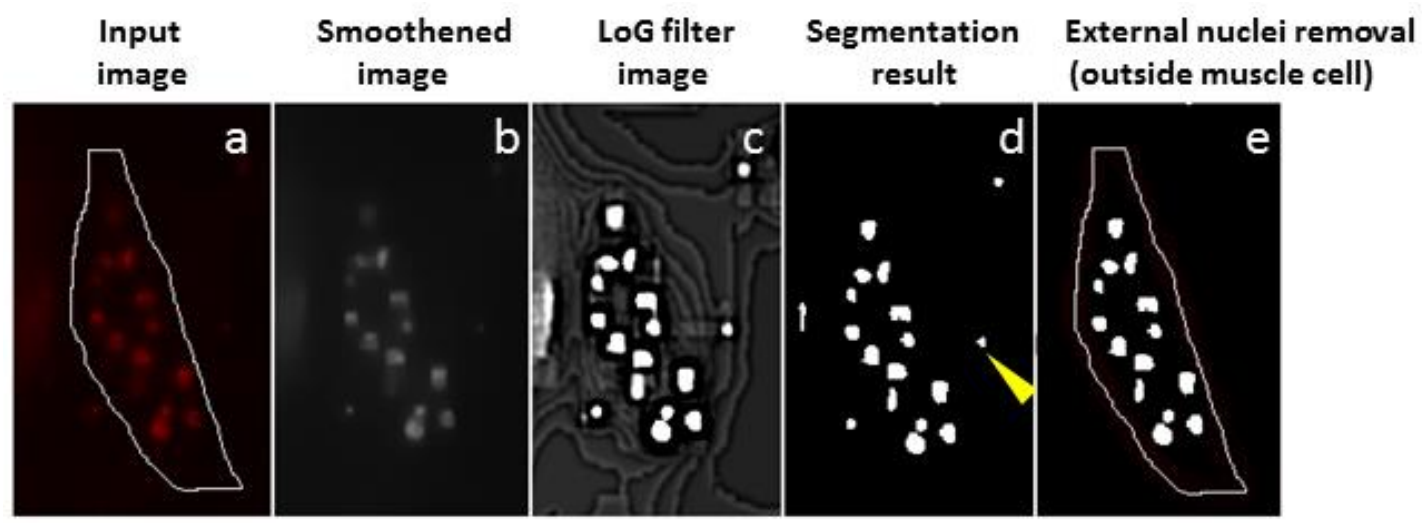

Figure 31. Results of different stages of myonuclear segmentation.

The figure shows the input image for nuclear segmentation (a) and the results of image smoothening using BEEPS (b), LoG filtering (c) and thresholding (d). The bright spots in (d) are the regions occupied by nuclei. The external nuclei located outside muscle cells are removed using muscle cell boundary (yellow arrowhead) as shown in (e). The white contour is the muscle cell boundary.

Since only the nuclei present inside the muscle cells are required for analysis; after obtaining the segmentation results, the external nuclei located outside the muscle boundary are removed, as shown in Figures 31e. In addition, external nuclei that appear inside muscles as a result of overlapping in image projections have to be removed from segmentation results. I will describe the classification technique used for removing external nuclei in the next section.

\subsubsection{Nuclear tracking and classification}

In order to remove the external nuclei from the segmentation results, I designed a methodology to classify external and internal nuclei based on their movements. There is a significant difference in the movement of these two types of nuclei. The external nuclei move faster $(25-37 \mu \mathrm{m} / \mathrm{hr})$ than internal nuclei $(0-12 \mu \mathrm{m} / \mathrm{hr})$. In my classification methodology, motion characteristic is used to differentiate between external and internal nuclei. First, I obtain the tracks of nuclei based on a proximity criterion and then classify these tracks on the basis of a cost function derived from nuclear movement in each track.

Various studies were done on tracking nuclei previously [126], [127]. However, these problems were customized for tracking nuclei during cell division. On the other hand, muscles have multiple nuclei and they don't undergo division. Combined with various issues in our dataset like indistinguishable nuclei due to their adhering to each 
other, missing nuclei in many time points due to movement of muscles during imaging; makes it a unique problem which cannot use the previous nuclei tracking techniques. In the following section, I'll discuss the approach on nuclear tracking and classification in detail.

\section{Step 1 : Generating tracks of nuclei}

First, I use connected component analysis on the nuclear regions extracted in previous section, to detect and label blobs [88], [96]. Each blob can contain one nucleus or multiple nuclei. I use these labelled nuclei to generate tracks. However, in our dataset, it is impossible to track a nucleus from beginning to the end of time series. As mentioned before, at certain time points the nuclei are so close to each other that it is difficult to distinguish them, and due to this issue, incomplete tracks of nuclei are generated. Therefore, instead of trying to track nuclei throughout the development, multiple smaller tracks were generated. For example, I am tracking a nuclei $n_{1}$ and the track number is $k_{1}$. At a time point $T=t, n_{l}$ comes close to another nucleus/group of nuclei and form a large clump of high intensity (individual nucleus not visible). At time point $T=t+1$, a nucleus separates from the group of nuclei. However, I am not sure whether it is nucleus $n_{1}$ or other nucleus from the group. In order to avoid this discrepancy, I call this separated nucleus $n_{2}$ and create a new track $k_{2}$. In this manner, multiple tracks of the same nucleus are created over a period of time. The tracks are created based on the nearest neighbour approach. For a nucleus at time point $t$, its nearest neighbour at time point $t+1$ is found using the minimum distance between centroids of nuclei as criterion. Similarly, for each nucleus at time point $t+1$, its nearest neighbour at time point $t$ is found. Three types of relationships were observed between nuclei in adjacent time points as shown in Figure 32a, a'. 
(a)

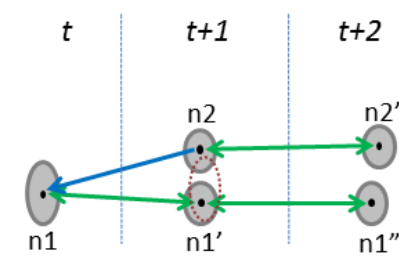

(b)

\footnotetext{
- Mutual relationship

One way relationship-Nucleus separates
}

One way relationship-Nucleus join

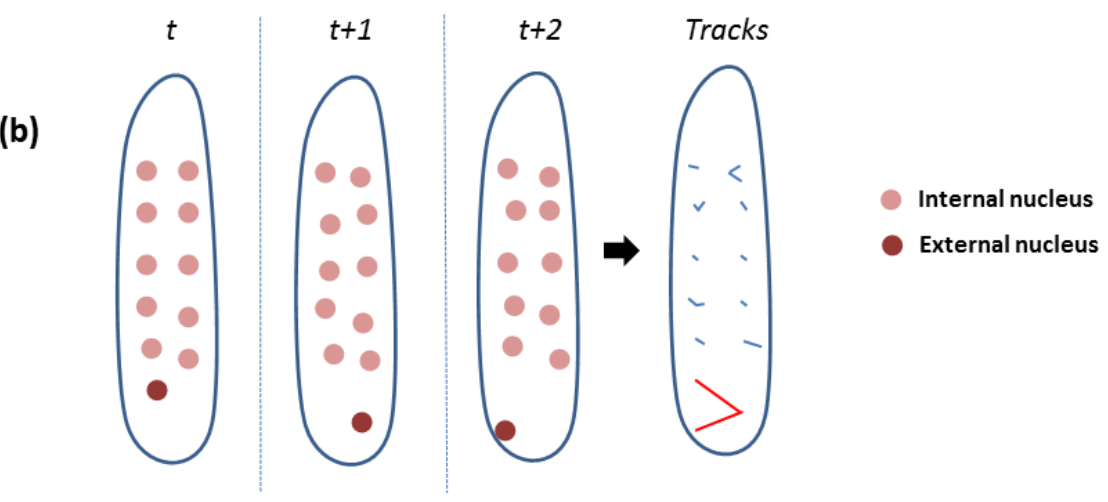

$\left(a^{\prime}\right)$

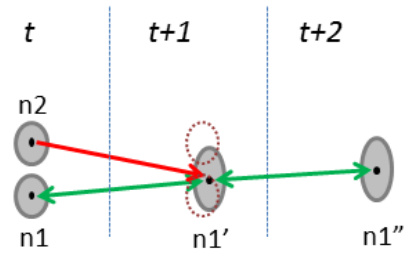

$\odot$ Nucleus

Figure 32. Nuclear tracking.

(a) Two nuclei which formed a clump $n l$ at time point $t$, separate at $t+1 . n 1$ and $n l$ ' are mutually closest to each other; therefore $n 1$ ' is added into the track containing $n 1$. Whereas, $n 2$ starts a new track. (a') Two internal nuclei $n l$ and $n 2$ at $t$ form a clump at $t+1$. Since $n 2$ does not have mutual closeness to $n 1$ '; the track containing $n 2$ terminates at $t$. Whereas, $n l$ forms a track which contains $n l$ ' and $n l$ '. (b) The diagram shows an example of tracks generated by nuclei movement between three time points. The movement of external nuclei is larger than the internal nuclei.

Case I: Nucleus $n l$ from time point $t$ is the nearest neighbour of nucleus $n l$ ' from time point $t+1$ and nucleus $n l$ ' from time point $t+1$ is the nearest neighbour of nucleus $n 1$ from time point $t$. Therefore, they have a mutual relationship, indicating that these two nuclei are the same.

Case II: Nucleus $n 1$ from time point $t$ is the nearest neighbour of nucleus $n 2$ from time point $t+1$, but nucleus $n 2$ is not the nearest neighbour to nucleus $n l$. This indicates that $n 1$ is a group of nuclei instead of single nucleus and $n 2$ is a nucleus that has broken off from group of nuclei $n l$ (Figure 32a). Here, 'broken off' refers to separation of nuclei which are very close to each other.

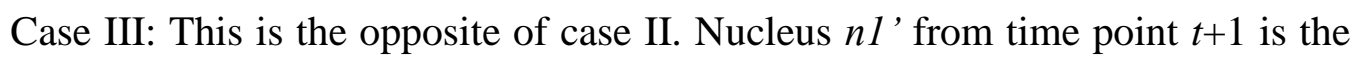
nearest neighbour to nucleus $n 2$ from time point $t$; however, nucleus $n 1$ ' is not the 
nearest neighbour of nucleus $n 2$ (Figure 32a'). This would indicate that $n 1$ ' is a group of nuclei that was formed by joining nucleus $n 2$ with another nucleus. Here, joining refers to two or more nuclei adhering to each other, making them indistinguishable.

Next, I'll explain the approach used to generate tracks from the relationships between nuclei in adjacent time points. At the first time point of time series stack, every nucleus starts a new track. If a nucleus has mutual relationship with another nucleus from the next time point (Case I), the track continues. However, if a nucleus has one way relationship with a nucleus in the next time point (Case III), its track terminates. New tracks are created when a nucleus does not have a mutual relationship with any nucleus in previous time point (Case II) (Figure 32a, a'). I represent the tracks in the form of a $\mathrm{T}$ by $\mathrm{X}$ table; where $\mathrm{T}$ is the time point and $\mathrm{X}$ is the total number of tracks. It contains the labels of nuclei. Each nucleus in a time point has a unique label. These unique labels are generated by finding connected components in the image (binary image containing nuclear region as 255 and background as 0) and labeling each component/nuclear region. The labels are assigned based on the location of nuclei along the y axis. Therefore, if there is a difference in the sequence of nuclei along y axis in subsequent time points, the same nuclei will have different labels. If external nuclei are also present, the labels of nuclei change. Track 2 in table 3 has different labels at many time points for the same nucleus. In the case of track 1 , the nucleus is closest to $\mathrm{x}$ axis and there are no nuclei in its proximity; therefore its label does not change in subsequent time points.

\begin{tabular}{|c|c|c|c|c|c|c|c|c|}
\hline \multirow{2}{*}{$\begin{array}{l}\text { Time, } \\
\text { aHE } \\
\text { [Hours] }\end{array}$} & \multirow{2}{*}{$\begin{array}{c}\text { Time } \\
\text { Frame } \\
\text { Number }\end{array}$} & \multicolumn{7}{|c|}{ Nuclear Tracks } \\
\hline & & Track 1 & Track 2 & Track 3 & Track 4 & Track 5 & Track 6 & Track 7 \\
\hline 36 & 87 & 100 & 102 & 103 & 104 & 108 & & \\
\hline 36.5 & 88 & 0 & 103 & 104 & 105 & 110 & 102 & 109 \\
\hline 37 & 89 & 0 & 102 & 103 & 104 & 107 & 0 & 111 \\
\hline 37.5 & 90 & 0 & 101 & 102 & 103 & 109 & 0 & 112 \\
\hline 38 & 91 & 0 & 102 & 103 & 104 & 108 & 0 & 0 \\
\hline
\end{tabular}

Table 3. Representation of nuclear tracks in tabular manner

Each column of the table is one track. The column contains the unique label of the nuclei which are part of the track. Zero value indicate that the track has terminated. Tracks shown in red were classified as external nuclei and their contours were removed from segmentation results. aHE: after Head Eversion 
The nuclei can be tracked accurately when their movement between adjacent time points is close to zero. However, that is not the case throughout pupal development. The movement of muscle cells varies during the development of pupa. Between 1240 hours after head eversion, due to fast movement of muscle cells, the distance covered by internal nuclei in adjacent frames is high (>10 pixels/12.4 $\mu \mathrm{m}$ distance between centroids of muscle cell in consecutive time points). Whereas, between 40 90 hours after head eversion, the distance moved by muscle cell is close to zero. In order to correctly track the nuclei between two consecutive time points, the position of nuclei are adjusted at one of the time points to compensate the movement of muscle cells. First, the amount of displacement muscle cell undergo is calculated by measuring the displacement of its centroid in two consecutive time points. For example, centroid of muscle cell moved $x$ pixels horizontally and $y$ pixels vertically between time point $t$ and $t+1$. Next, the image at time point $t$ is translated horizontally by $x$ pixels and vertically by $y$ pixels, so that the nuclei at time point $t$ and $t+1$ align with each other. This alignment facilitates the tracking process by reducing the number of incorrect matching of nuclei between consecutive time points.

\section{Step 2 : Distinguishing the internal nuclei tracks from external nuclei tracks}

As mentioned before, the movement of internal nucleus is much smaller than the movement of external nucleus. I exploit this property to classify these two types of nuclei. Intuitively, if a track belongs to an external nucleus, then the average movement of nucleus between consecutive frames should be higher as compared to a track which belongs to an internal nucleus. A schematic diagram in figure $32 \mathrm{~b}$ shows the difference between the track of an internal and external nucleus. Therefore, I design a cost function which is an indicator of the nuclei motion. For a track $x$ of length $n_{x}$ which starts at time point $t_{s}$ and ends at time point $t_{e}$, the cost function $M(x)$ is given as

$$
M(x)=\frac{1}{n_{x}} \sum_{i=t_{s}+1}^{i=t_{e}} D_{i} *\left(1-O_{i}\right)
$$

$D_{i}$ is the distance between centroid of nuclei in consecutive time points and $O_{i}$ is the percentage overlap between nuclei in consecutive time points. Higher value of cost function indicates higher possibility of the track belonging to an external nucleus and 
vice versa. The overlap factor $\left(\mathrm{O}_{\mathrm{i}}\right)$ increases the cost when the overlap between nuclei is low.

After obtaining the cost function for every track, they are classified on the basis of a cost cut-off. Tracks whose cost function is higher than a threshold $\lambda$ are classified as tracks of external nuclei. The external nuclei from these tracks are removed from the segmentation results (Figure 33). Since, the position of nuclei is normalized according to the movements of muscle cells during calculations; the threshold $\lambda$ should be same for every time series stack. The value of threshold is obtained by performing accuracy tests on training datasets; which will be discussed in the results section.

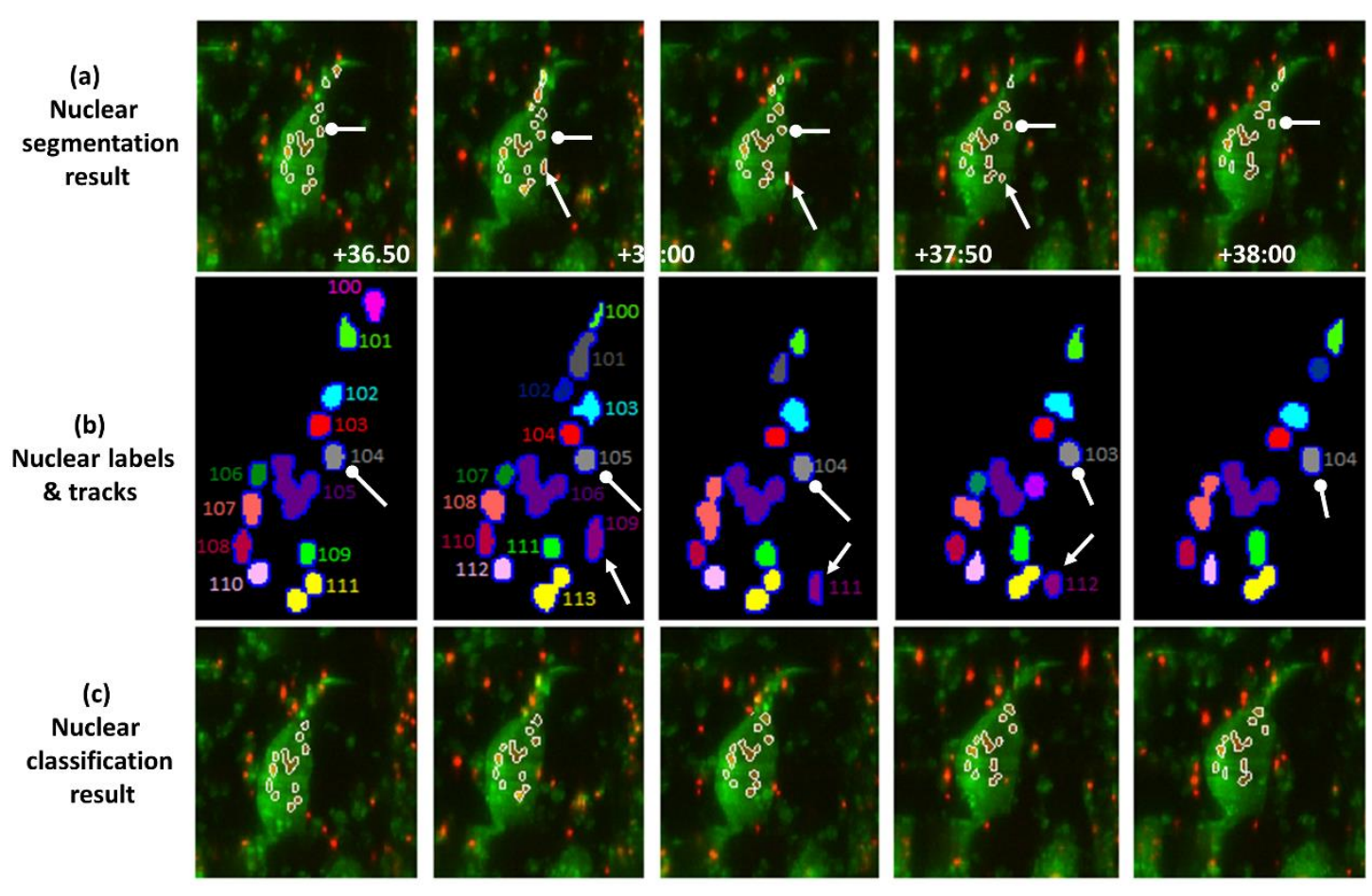

Figure 33. Results of nuclear classification.

(a) The images show the result of nuclear segmentation for five time points selected from a pupa expressing Atg9 RNAi. DIOM muscle from 3rd segment of pupa abdomen was used for this analysis. The segmented nuclei contain both internal and external nuclei. The white arrow and oval arrow indicates an external and internal nucleus. (b) The labels of nuclei at each time point are shown. Nuclei which have same colour in subsequent time points belong to same track. (c) The images show results of nuclear classification. The contours of classified external nuclei are removed from the segmentation results. 


\subsubsection{Nuclear spatial pattern feature}

As mentioned before, during certain time points, it is impossible to identify the location of each nucleus because multiple nuclei are lying very close to each other and form a large clump of high intensity. In these cases, extraction of nuclear region generates a blob (connected components) which contains many nuclei. For this reason, it was not possible to use point pattern analysis [116] to study nuclear distribution and had to design a different approach which uses complete nuclear structure inside muscles as compared to only centroids for nuclear pattern feature generation. I designed three new features which quantify different types of nuclear distributions i.e. nuclear spatial density, longitudinal migration index and lateral displacement index. Before analyzing the nuclear distribution, the nuclear structure is rearranged along the straightened medial axis of the muscle cell.

\section{Rearrangement of nuclei in straightened muscle cell}

Aligning the nuclei along the straightened medial axis of muscle cell helps to provide a spatial reference for comparing nuclei from different samples. I assume that the medial axis of muscle cell is straight and adjust the position of nuclei according to the changes in the curvature of the medial axis (Figure $34 \& 35 \mathrm{a}-\mathrm{a}$ "). The straightening algorithm is as follows:

1. Find the centroid of a nucleus.

2. Find the smallest distance $(d x)$ to the centroid from the medial axis of muscle cell. Let the point at the medial axis which has the least distance from the centroid be $m$.

3. Find the length of medial axis $(d y)$ between the start of medial axis and point $m$.

4. Find the angle $(\alpha)$, i.e. the angle between the tangent at point $m$ of medial axis and y axis.

5. Assuming that the start point of straightened medial axis lies at $x=\mathrm{X}, y=0$, then the new coordinate of point $m$ is $P(X, d y)$ and the centroid of the nucleus is $P(X \pm d x, d y) . \mathrm{X}$ is a constant number such that $X \pm d x$ is never negative. The sign in $X \pm d x$ depends on whether the nucleus is located on left or right side of medial axis. 
6. Next, Translate the nucleus to point $P(X \pm d x, d y)$ and rotate the nucleus by an angle $\alpha$.

7. Repeat steps 1-6 for each nuclei inside the muscle cell.
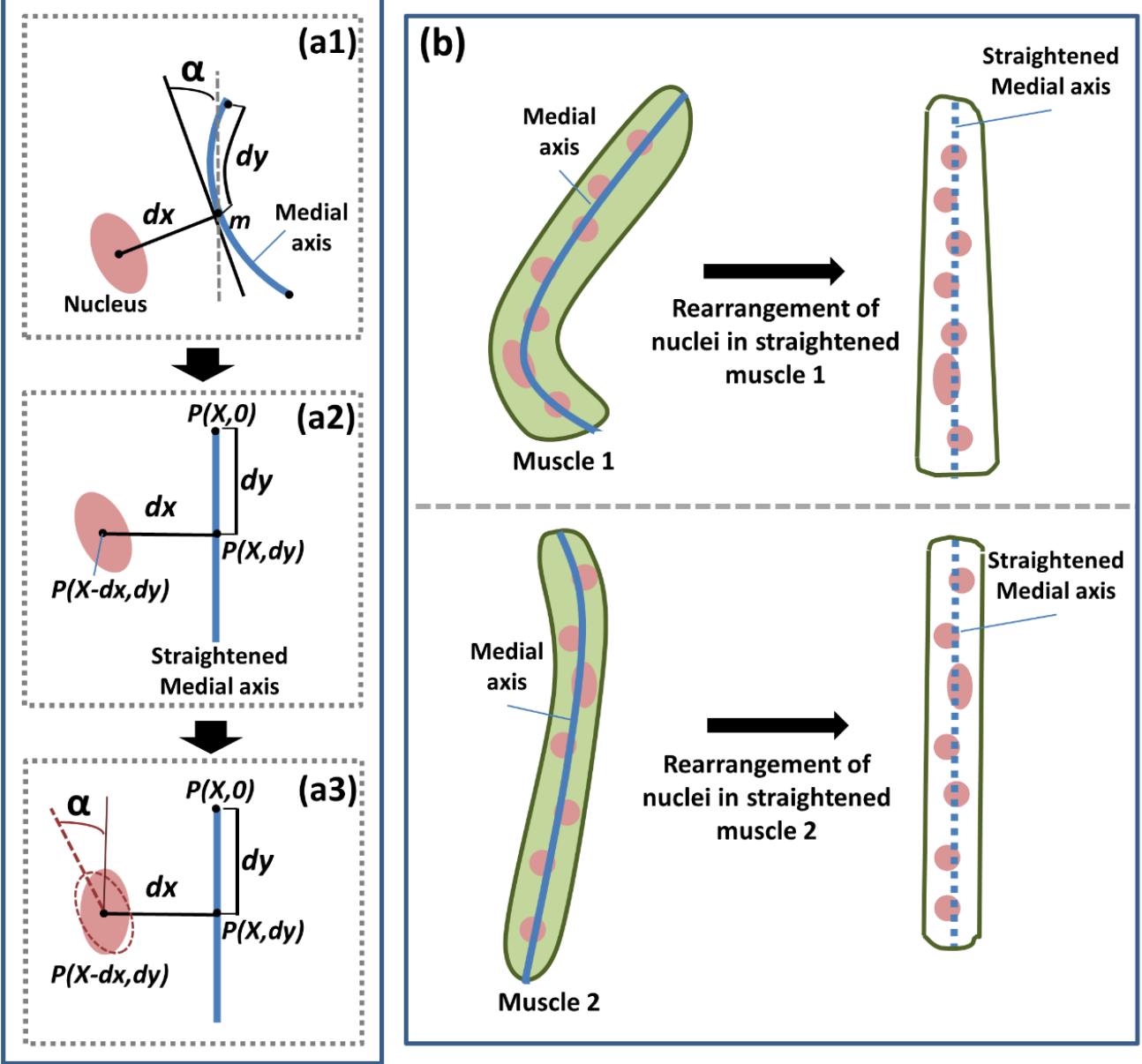

Figure 34. Rearrangement of nuclei in straightened muscle cell for comparing nuclei from different samples.

(a1-a3) The figure shows various steps involved in rearrangement of a nucleus along straightened muscle medial axis i.e. calculation of new coordinates of nucleus and medial axis (a1), translation of nucleus to new coordinate (a2) and rotation of nucleus about its centroid (a3). (b) Rearranged nuclei along medial axis are shown for two muscles with different shapes. 

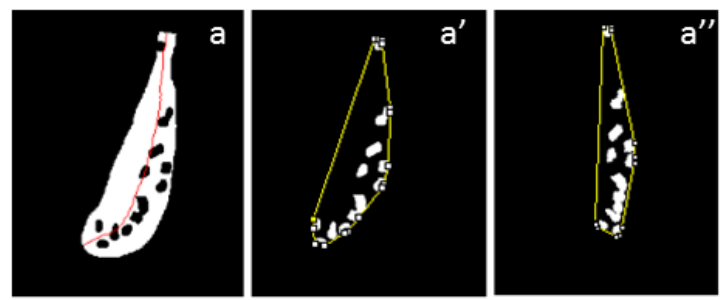

$\mathrm{ND}=\mathbf{0 . 7 7}$

$\mathrm{ND}=0.52$

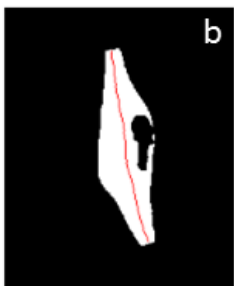

$\mathrm{ND}=\mathbf{0 . 1 1}$

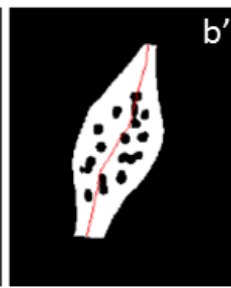

$\mathrm{ND}=0.38$

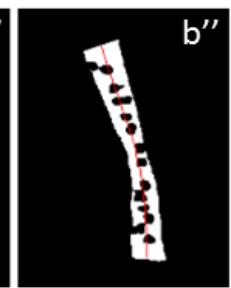

$\mathrm{ND}=\mathbf{0 . 7 2}$

Figure 35. Example showing differences in nuclear spatial density due to change in cell size.

(a-a'") ND : Nuclear density. The figure shows straightening of nuclei with respect to the medial axis of nuclei. It compares the nuclear density index for straightened and un-straightened nuclei. (b-b") The value of nuclear spatial density is lower for densely packed nuclei (b) as compared to evenly distributed nuclei (b").

\subsubsection{Nuclear spatial density}

As mentioned earlier, it has been previously shown that there is an association between nuclear localization and muscle mass change. To confirm this hypothesis, I designed a feature that measures the nuclear spatial density with respect to the cell size.

$$
\text { Nuclear spatial density }=\frac{\text { Area of convex hull of nuclei }}{\text { Area of muscle cell }}
$$

A straightened nuclear structure is used to calculate the convex hull. The convex hull of a nuclear region is the smallest convex set which contains that region [128]. If nuclear spatial density is close to 1 , then the nuclei are located close to the muscle boundary and are distributed more evenly. Alternatively, a low value of nuclear density indicates that the nuclei formed a cluster and occupied a small part of the muscle cell. In figure 35, the sample $b$ has the smallest nuclear density, resulting from clustering of the nuclei. In sample $b$ ', although the nuclei are more spread out, the nuclear density is not as high as in sample $b^{\prime \prime}$. This is because sample $b$ ' has a larger cell size than sample $b ”$. 
(a)

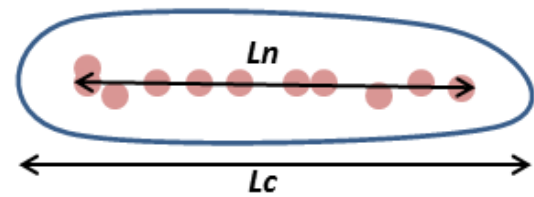

(b)

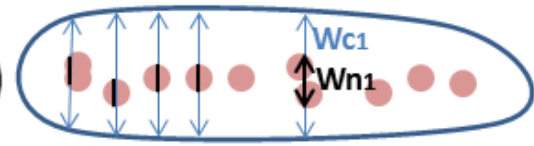

Figure 36. Schematic diagram explaining the derivation of (a) Normalized longitudinal migration index.

(b) Normalized lateral displacement index.

\subsubsection{Longitudinal nuclear spread}

Longitudinal nuclear spread characterizes the polar and anti-polar migration of nuclei. It is defined as the distance between the extremes of the nuclei along the medial axis and denoted as $L_{n}$. The normalized migration of nuclei along the medial axis/normalized longitudinal nuclear spread $N M_{l o n}$ is defined as:

$$
N M_{\text {lon }}=L_{n} / L_{c}
$$

where $L_{n}$ is the longitudinal nuclear spread and $L_{c}$ is the length of the muscle cell (Figure 36a). A high $N M_{\text {lon }}$ indicates that the nuclei are close to the poles of the muscle cell and a low value indicates that they are far.

\subsubsection{Lateral nuclear spread}

Lateral nuclear spread characterizes the movement of nuclei away from the medial axis of muscle during late stages of pupal development. The lateral nuclear spread $M_{\text {lat }}$ is defined as:

$$
M_{\text {lat }}=\frac{1}{u} \sum_{i=1}^{i=u} W_{n_{i}}
$$

where $W_{n_{i}}$ is the width of the nuclear structure at the $i^{t h}$ location on the medial axis, and $u$ is the number of samples taken along medial axis (Figure 36b). All samples are collected at equal interval along medial axis. A high $M_{\text {lat }}$ indicates an increase in the distance between nuclei along the width of muscle i.e. two-row formation of nuclei. 
In order to quantify the influence of muscle mass change on lateral displacement of nuclei, I also designed normalized lateral nuclear spread. Normalized lateral nuclear spread $N M_{\text {lat }}$ is defined as:

$$
N M_{\text {lat }}=\frac{1}{u} \sum_{i=1}^{i=u} W_{n_{i}} / W_{c_{i}}
$$

where $W_{c_{i}}$ is the width of muscle cell at the $i^{\text {th }}$ location on the medial axis. A high $N M_{\text {lat }}$ indicates that the nuclei are close to the boundary of the muscle cell. A few examples of the values of longitudinal nuclear spread and lateral nuclear spread are shown in Figure 37.
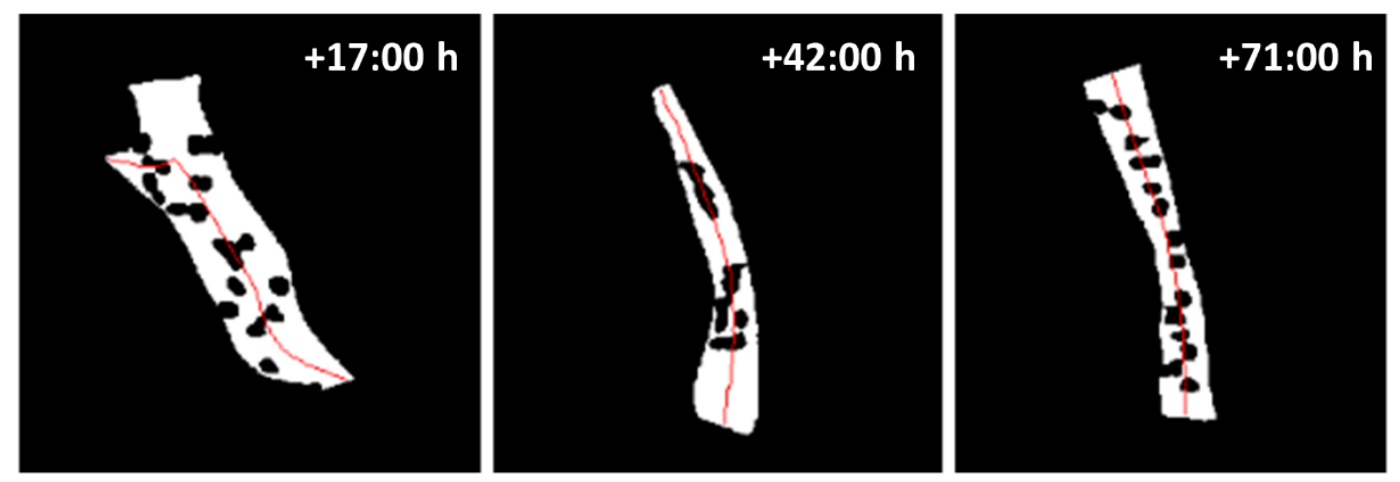

\begin{tabular}{|c|c|c|c|c|}
\hline $\begin{array}{c}\text { Time point } \\
\text { (h) }\end{array}$ & $\begin{array}{c}\text { Longitudinal } \\
\text { nuclear spread }\end{array}$ & $\begin{array}{c}\text { Lateral nuclear } \\
\text { spread }\end{array}$ & $\begin{array}{c}\text { Normalized } \\
\text { longitudinal } \\
\text { nuclear spread }\end{array}$ & $\begin{array}{c}\text { Normalized } \\
\text { lateral nuclear } \\
\text { spread }\end{array}$ \\
\hline+17 & 129 & 14.2 & 0.76 & 0.32 \\
\hline+42 & 105 & 7.53 & 0.59 & 0.36 \\
\hline+71 & 157 & 6.76 & 0.87 & 0.30 \\
\hline
\end{tabular}

Figure 37. Comparison of nuclear pattern features of a muscle at three different time points.

The muscle shown in the figure is from a control samples. At $+71 \mathrm{~h}$, the muscle has highest Mlon and NMlon because the nuclei are closer to the poles of the muscle cell than at other time points. Whereas, the muscle has highest Mlat at $+17 \mathrm{~h}$; because the nuclei are more spread out along the width of muscle. The value of NMlat is approximately similar at three time points. This indicates that at these three time points, the spread of nuclei with respect to width of muscle is similar. 


\subsection{Summary}

In this chapter, the algorithm for nuclear distribution analysis was discussed. Two major contributions of this chapter are:

1. Nuclei segmentation results were improved by introducing a tracking based nuclei classification algorithm. The nuclei classification algorithm exploits the motion behavior of nuclei to classify them. It is also robust enough to reduce classification errors during large movements of the muscle cell.

2. Multiple nuclear spatial pattern features were designed, each catering to a specific type of phenotype. The nuclear spatial density index measure how densely the nuclei are packed; however, they cannot quantify the localization of the nuclei in the muscle cell. To accomplish this, I designed new features that can quantify the distribution of nuclei along the medial axis and along the width of the muscle cell i.e. the longitudinal migration index and lateral displacement index. 


\section{Chapter 6 Experimental Results}

\subsection{Testing and Validation of Muscle Segmentation}

\subsubsection{Segmentation evaluation}

I evaluated the performance of segmentation using the ground truth produced manually using the segmentation editor tool of ImageJ [129]. During ground truth creation, each cell in an image was labeled separately to avoid any discrepancy due to closeness of cells. An in-house ImageJ plugin was used to measure the performance of new segmentation method. This plugin takes two inputs: a time series stack of binary segmentation results and a stack of labeled ground truth for the same time points. Both stacks should have the same dimensions. As a cell is labeled with the same id in subsequent time series images, it is possible to compare the performance of segmentation across different muscle cells. Three parameters were used to evaluate the performance of the segmentation algorithm namely Recall, Precision and the Fmeasure.

$$
\begin{aligned}
& \text { Recall }=\mathrm{TP} /(\mathrm{TP}+\mathrm{FP}) \\
& \text { Precision }=\mathrm{TP} /(\mathrm{TP}+\mathrm{FN}) \\
& \text { F-measure }=2 * \frac{(\text { Recall } * \text { Precision })}{(\text { Recall }+ \text { Precision })}
\end{aligned}
$$

True positive (TP) are the pixels correctly segmented as muscle cell, true negative (TN) are the pixels correctly segmented as non-muscle (background and debris), false positive (FP) are the non-muscle pixels which were incorrectly segmented as muscle cell and false negative (FN) are the muscle cell pixels which were incorrectly segmented as non-muscle.

I segmented six muscles at different developmental stages for evaluation. For each muscle, the user manually drew the muscle contour at a chosen time point and the subsequent time points were segmented by the algorithm. Segmentation was carried out using the following parameters $\mathrm{T}=1 / 25$. The performance evaluation results are 
discussed in the next section.

\subsubsection{Segmentation performance assessment}

Figure 38 a-c shows the segmentation results of three different muscle cells at multiple time points.

a
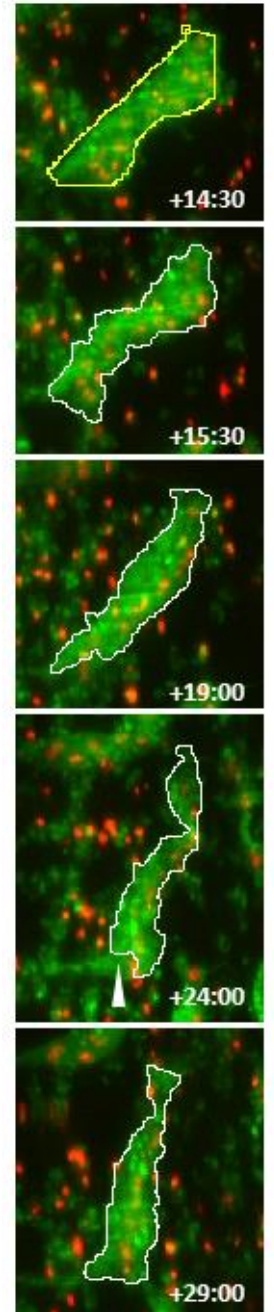

b

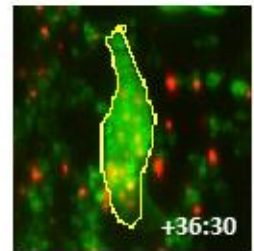

$+36: 30$
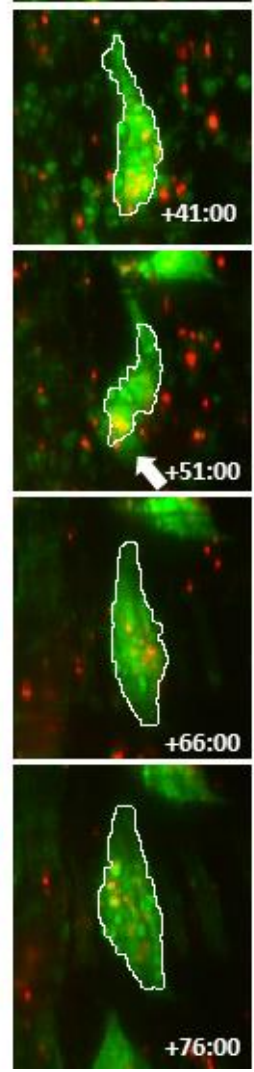

c
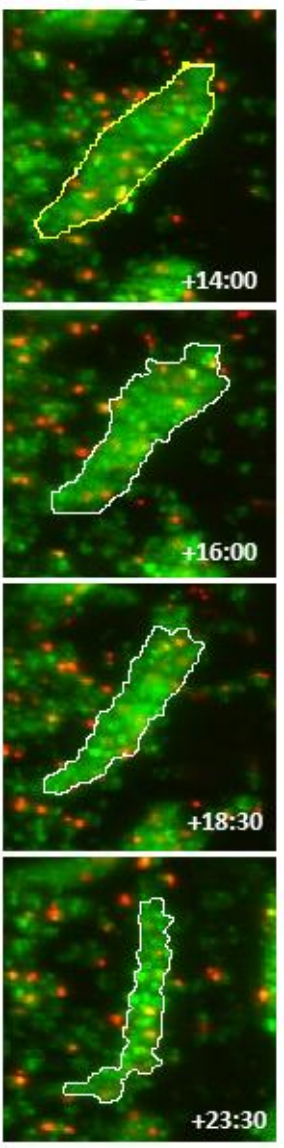

$+23: 30$
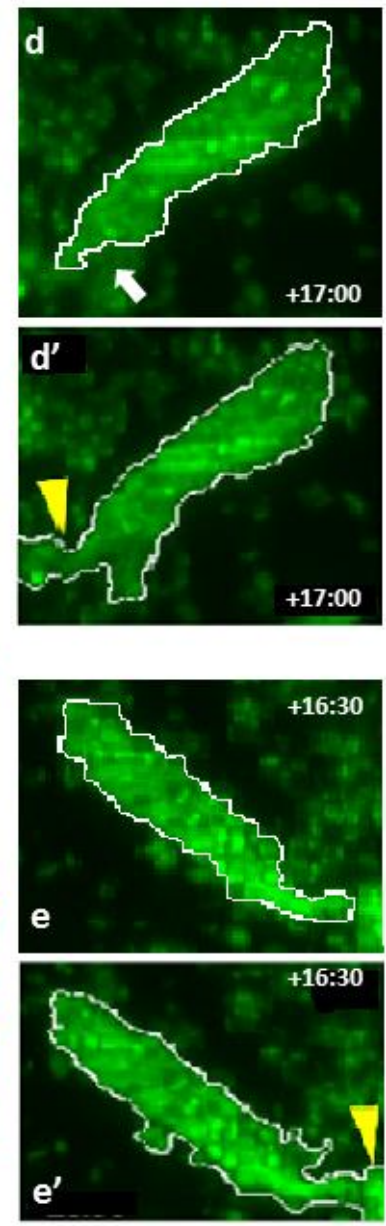

Figure 38. Segmentation evaluation.

(a \& c) Segmentation results of muscles from the early developmental stage. (b) Segmentation results of muscles from the late developmental stage. White arrowhead indicates the incorrect segmentation due to large movement of muscles. White arrow indicates the incorrect segmentation due to low intensity region. The muscle contours in the first row of column (a-c) were initialized by the user. (d - e') show the comparison of my method (d, e) with a previous study (d', e') [77]. Yellow arrowhead shows the incorrect segmentation result due to adjoining debris (d') or another muscle cell (e'). 
The advantage of watershed based segmentation method is that the results adhere to the actual boundary of the muscle cell instead of leaking out to the debris as shown in Figure 38 d, e. Figure 38 d', e' show the results of a previous study where leakage of boundary was observed (indicated by yellow pointer) [77]. Improved results were obtained by my segmentation technique because the muscle boundary at $t$ time point is restricted to be close to the muscle boundary $B_{t-1}$ at $t-1$ time point. There are two reasons for this behavior. First, due to level 1 of region classification, regions which do not overlap with the muscle cell at $t-1$ are removed from muscle class irrespective of their intensity. Secondly, by comparing shapes at level 2, any boundary region whose edge is far from $B_{t-1}$ or has edge with different orientations from $B_{t-1}$ was discarded. The edge confidence component of the dissimilarity metric increases the robustness of method by ensuring that muscle regions with high confidence edges are not falsely reclassified because of slightly high shape dissimilarity. Thus, by balancing the temporal shape based distance and edge confidence feature in region classification algorithm, high segmentation accuracy was obtained. The segmentation evaluation results are as follows: average recall $=80.1 \pm 15.9 \%$, average precision $=89.3 \pm 6.5 \%$. My method was able to achieve an accuracy (F-measure) of $83.5 \pm 11.5 \%$. To test the functionality of the algorithm, I performed the evaluation test for different scenarios by altering the structure of muscle segmentation algorithm. I calculated the performance of segmentation algorithm with only one feature i.e. either edge confidence or oriented chamfer distance. As expected, the accuracy of algorithm goes down drastically for both without edge confidence $(78.2 \pm 16.8 \%)$ and without oriented chamfer distance $(48.8 \pm 38.8 \%$ ) (Table 4). Segmentation with only chamfer distance feature was affected by the movement of muscles during early stages of metamorphosis; whereas edge confidence based segmentation resulted in poor performance because of the missing canny edges. It shows that both features contribute to the improvement in time series segmentation of muscle cells.

In Figure 39, the F-measure values at each time point were plotted for four samples. During early pupal development, muscle cells were segmented for only 20-30 time points. Due to large amount of debris during this stage, the rate of error in segmentation was high. As opposed to early stages, the amount of debris is low during late stage i.e. after $+50 \mathrm{~h}$. Therefore, more number of time points were segmented with only one muscle boundary initialization. The performance of the segmentation method also 
reduces when the intensity of some sections of the muscle cell are extremely low as compared to the rest of the muscle cell (Figure 39 - sample 4). The algorithm was compared against the semi-automated level set based muscle segmentation algorithm from a previous study [77]. For the same datasets, the latter gave an average recall of $87.1 \pm 12.9 \%$, an average precision of $75.4 \pm 19.8 \%$ and an average accuracy of $79.2 \pm 16.8 \%$ (Table 4). On comparison, the recall was higher for the previously published method; however my algorithm outperformed in terms of the accuracy. The leakage of boundary into the debris of the muscles resulted in low precision in previous study on muscle segmentation discussed above. I also compared the performance of watershed based segmentation algorithm with two more segmentation techniques i.e. levelset and kmeans. In levelset segmentation, I performed two tests i.e. the 0-levelset contour could move only inside and only outside [95]. In case of inside movement of contour, due to movement of muscles after few time points, the overlap of contour with the muscle gradually reduces. This resulted in disappearance of contour and low segmentation accuracy especially recall $(40 \pm 24.8 \%)$. Whereas, in case of outward movement of levelset contour, the contour moved into the adjoining debris and could not be restricted to the muscle. This resulted in very low precision of segmentation $(44.3 \pm 28.5 \%)$. As compared to levelset, kmeans suffered from low intensity region in the muscle cell [130]. The low intensity region in muscle were falsely classified as debris by kmeans clustering; resulting in low recall $(74.5 \pm 21 \%)$. Therefore, among all the segmentation techniques discussed above, watershed based segmentation algorithm gave the best accuracy on muscle dataset. 


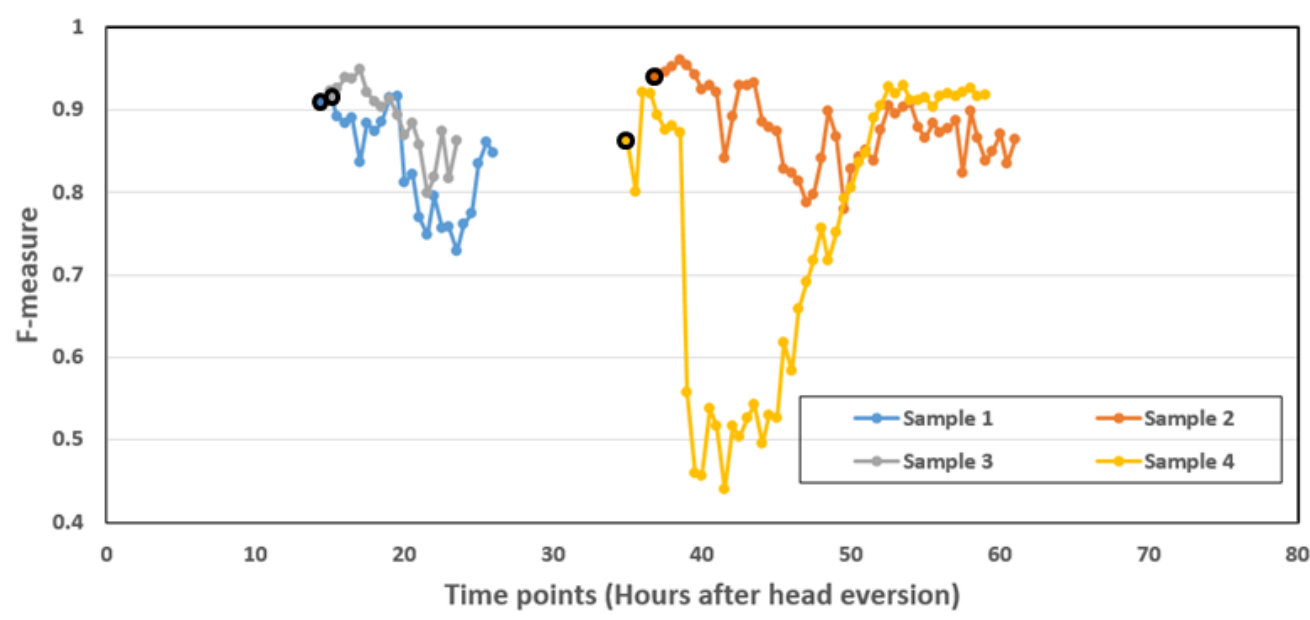

Figure 39. Graph comparing F-measure at different stages of development for four muscle cells.

The large black dots indicate the time point segmented after manual initialization of muscle boundary. Sample 4 shows significant reduction in accuracy around $+40 \mathrm{~h}$ due to out of focus muscle cell.

\begin{tabular}{|c|c|c|c|}
\hline & $\begin{array}{c}\text { Average Recall } \\
\mathbf{( \% )}\end{array}$ & $\begin{array}{c}\text { Average } \\
\text { Precision (\%) }\end{array}$ & $\begin{array}{c}\text { Average F- } \\
\text { measure (\%) }\end{array}$ \\
\hline Watershed based Method & $80.1 \pm 15.9$ & $89.3 \pm 6.5$ & $83.5 \pm 11.5$ \\
\hline $\begin{array}{c}\text { Watershed based Method-Edge } \\
\text { confidence feature only }\end{array}$ & $47.3 \pm 39.4$ & $55.5 \pm 41.8$ & $48.8 \pm 38.8$ \\
\hline $\begin{array}{c}\text { Watershed based Method-Oriented } \\
\text { chamfer distance feature only }\end{array}$ & $76.4 \pm 16.1$ & $83.2 \pm 17.9$ & $78.2 \pm 16.8$ \\
\hline Chinta et al & $87.1 \pm 12.9$ & $75.4 \pm 19.9$ & $79.2 \pm 16.8$ \\
\hline Kmeans & $74.5 \pm 21$ & $90.8 \pm 8.8$ & $79.6 \pm 17.9$ \\
\hline Level Set (Inward) & $40 \pm 24.8$ & $77.3 \pm 20.1$ & $48.3 \pm 23.7$ \\
\hline Level Set (Outward) & $92.9 \pm 7.1$ & $44.3 \pm 28.5$ & $52.7 \pm 30.1$ \\
\hline
\end{tabular}

Table 4. Comparison of the muscle segmentation performance of my method and a previous studies.

\subsection{Evaluation of Nuclear Segmentation and Classification}

\subsubsection{Nuclear segmentation}

I evaluated the performance of segmentation at object level rather than pixel level. The values of false negative, false positive and true positive were manually counted by comparing the segmentation results with original image. False positive (FP) is the count of segmented objects which were not nuclei. True positive (TP) is the count of 
segmented objects which were nuclei. False negative $(\mathrm{FN})$ is the count of nuclei which were not segmented. The segmentation results were evaluated for 140 time points that were selected from two Atg9 samples. Following two parameters were used for evaluation:

$$
\begin{aligned}
& \text { False negative rate }=\mathrm{FN} /(\mathrm{FN}+\mathrm{TP}) \\
& \text { False positive rate }=\mathrm{FP} /(\mathrm{FP}+\mathrm{TP})
\end{aligned}
$$

The false negative rate is the ratio of false negative and the total number of actual nuclei; whereas, the false positive rate is the ratio of false positive and the total number of nuclei segmented.

The performance of thresholding segmentation with LoG filtering is compared with thresholding (Otsu) without LoG filtering in Table 5. As shown in Table 5, LoG based method has zero false negative rate and 0.024 false positive rate. It means that LoG based method correctly segments every nuclei; however it also segments some nonnuclei high intensity spots (imaging noise). In comparison, Otsu thresholding without LoG filtering has false negative rate of 0.11 which is not suitable for spatial pattern analysis. The results of LoG based segmentation without bi-exponential smoothening is also shown in Table 5. The false positive rate is higher in the case of segmentation without smoothening as compared to with smoothening. This verifies that smoothening of our images is necessary to remove imaging noise. 


\begin{tabular}{|c|c|c|c|c|c|}
\hline & $\begin{array}{c}\text { True } \\
\text { positive }\end{array}$ & $\begin{array}{c}\text { False } \\
\text { positive }\end{array}$ & $\begin{array}{c}\text { False } \\
\text { negative }\end{array}$ & $\begin{array}{c}\text { False } \\
\text { positive } \\
\text { rate }\end{array}$ & $\begin{array}{c}\text { False } \\
\text { negative } \\
\text { rate }\end{array}$ \\
\hline $\begin{array}{c}\text { Otsu thresholding without } \\
\text { LoG filter }\end{array}$ & 846 & 0 & 108 & 0 & 0.11 \\
\hline $\begin{array}{c}\text { LoG based segmentation } \\
\text { with BEEPS smoothening }\end{array}$ & 1180 & 29 & 0 & 0.024 & 0 \\
\hline $\begin{array}{c}\text { LoG based segmentation } \\
\text { without BEEPS } \\
\text { smoothening }\end{array}$ & 1180 & 334 & 0 & 0.221 & 0 \\
\hline
\end{tabular}

Table 5. Nuclear segmentation performance evaluation.

\subsubsection{Nuclear classification}

$\lambda=7$ is used for nuclei classification in our dataset. In order to find the correct $\lambda$ for our dataset, a training dataset was used to calculate the performance of classification for different thresholds. I calculated following parameters for classification performance evaluation: accuracy, false positive rate and false negative rate.

$$
\text { Accuracy }=(\mathrm{TP}+\mathrm{TN}) /(\mathrm{TP}+\mathrm{FP}+\mathrm{TN}+\mathrm{FN})
$$

The false negative, false positive, true negative and true positive were calculated by comparing the classification results with a ground truth which was generated manually using FMAj. False positive (FP) is the count of external nuclei which were falsely classified as internal nuclei. True positive (TP) is the count of correctly classified internal nuclei. False negative (FN) is the count of internal nuclei falsely classified as external nuclei. True negative (TN) is the count of correctly classified external nuclei. The performance evaluation was done for 6 different muscle cells from different genotypes i.e. two samples from control, $\operatorname{Atg} 9$ and $\operatorname{Atg} 12$; data was generated from at least 50 time points per muscle cell. I visualized accuracy, false positive rate and false negative rate for these 6 muscles in a graph as shown in Figure 40a-c. The threshold at which accuracy was highest and error rates were minimum was selected for classification of nuclei (Shown by black dotted line) i.e. $\lambda=7$. 
Thus, the performance of the nuclear classification algorithm is measured at $\lambda=7$, for the same 6 muscles which were used for calculation of $\lambda$. The results are as follows: average accuracy $=96.9 \pm 1.2 \%$, average false positive rate $=1.2 \pm 0.2 \%$, average false negative rate $=2.1 \pm 1.3 \%$. A comparison of nuclear classification performance evaluation during different stages of pupal development is shown in Figure 40d-e. It can be observed that the accuracy of nuclear classification suffers due to the large movements of internal nuclei between 20 hours and 50 hours after head eversion. Whereas, during later stages of pupal development, the reduction in movement of internal nuclei results in high accuracy and low false positive and negative rates.

(a)

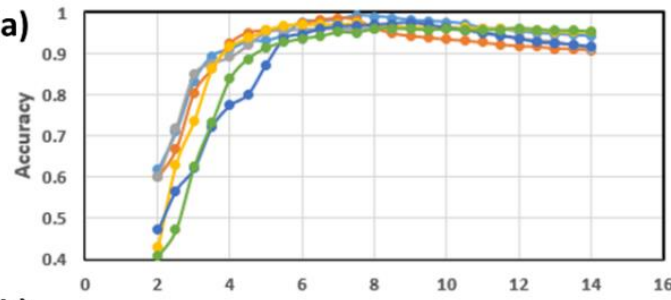

(b)
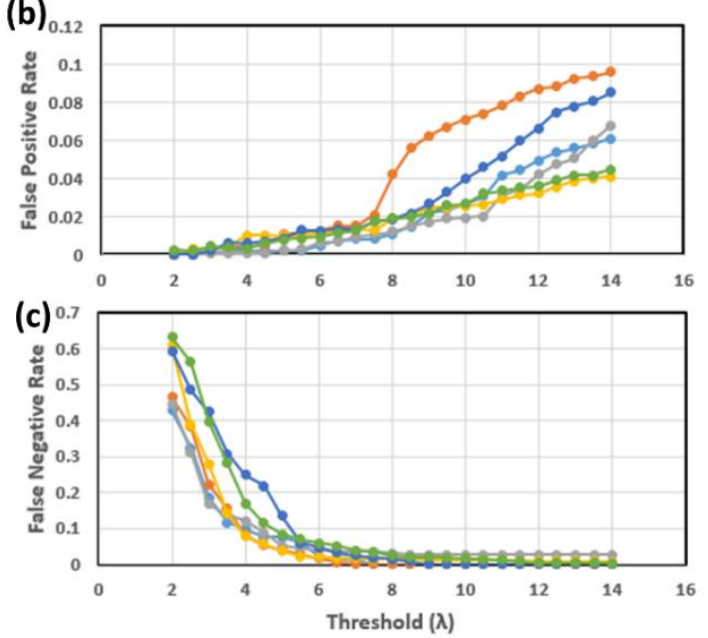
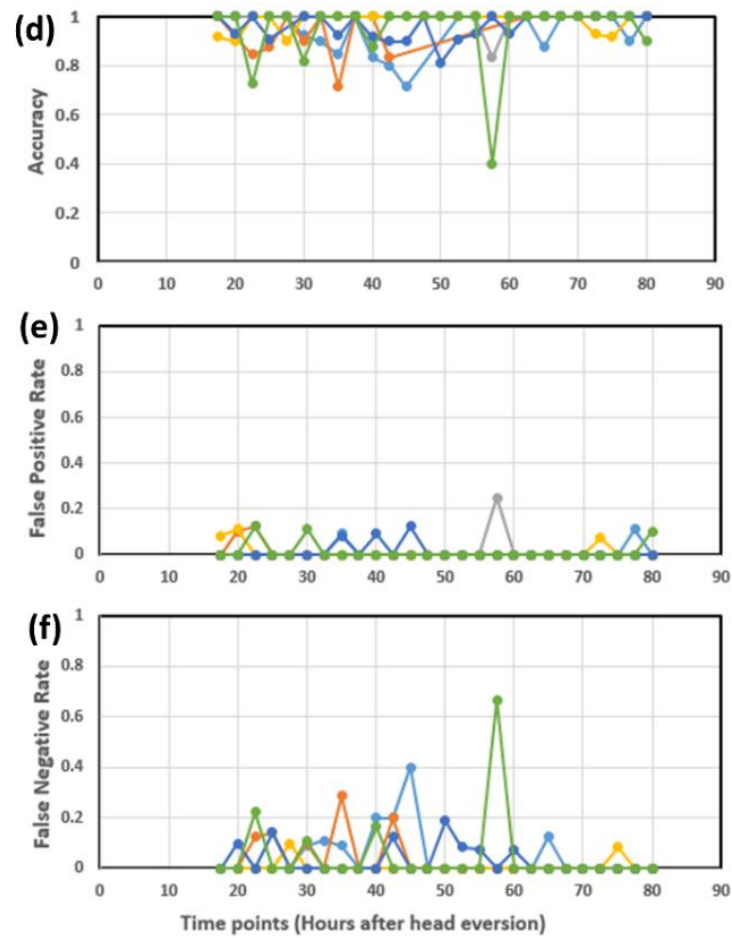

Figure 40. Performance evaluation of nuclear classification at different threshold values and different development stages.

(a-c) The graphs compare the performance of nuclear classification at different thresholds using following parameters i.e. accuracy (a), false positive rate (b) and false negative rate (c). The black dotted line indicate the threshold value at which accuracy is highest and error rates are lowest. This threshold value is used for nuclear classification. (d-f) The graphs compare the performance of nuclear classification at different stages of pupal development using following parameters i.e. accuracy (d), false positive rate (e) and false negative rate (f). Large movements of internal nuclei results in low accuracy 
and high error during between $+20 \mathrm{~h}$ and $+50 \mathrm{~h}$. In comparison, accuracy is high after $+50 \mathrm{~h}$ due to slow movement of internal nuclei.

The performance of nuclear classification method was compared with another tracking based technique. The MTrack2 application from ImageJ can track objects in 2D over time based on parameters like nuclei larger/smallest size, nuclei velocity and track length [131]. In order to use it for classification, I restricted the velocity of nuclei to $12 \mu \mathrm{m}$ which is the maximum velocity observed in internal nuclei. Thus, the tracks found by MTrack2 application will belong to internal nuclei. The nuclei which were not a part of any track were classified as external nuclei. The results from classification of 135 time points from Atg9 samples for both MTrack2 and my method is shown in the table 6. It shows that my method performs better than Mtrack2 in nuclear classification by $5.7 \%$.

\begin{tabular}{|c|c|c|c|c|c|}
\hline Method & TP & TN & FP & FN & Accuracy (\%) \\
\hline My method & 1011 & 117 & 9 & 12 & 98.2 \\
\hline MTrack2 & 992 & 71 & 55 & 31 & 92.5 \\
\hline
\end{tabular}

Table 6. Comparison of performance of my nuclear classification with MTrack2

\subsection{Phenotypic Analysis Results}

In a previous study by our lab, targeted genetic perturbation analysis was done for a set of genes [114]. Qualitative analysis revealed that mutation in some of these genes resulted in changes in the muscle morphology and abnormal myonuclei distribution during metamorphosis. In the current study, we have further analyzed a new set of genes to find their role in muscle remodeling during metamorphosis. Also, an image processing tool (FMAj) was used to quantify the phenotypes for genes from both studies. Hereafter, we will use head eversion as a reference to compare different samples and the time will be represented as hours (h) after head eversion. First, i'll discuss the contributions of our muscle phenotypic analysis framework and in what way it differed from previous analyses.

As discussed before, very few studies have been done on understanding the genes 
regulating the mechanisms of muscle development during Drosophila metamorphosis using in vivo analysis [54], [73], [74], [76], [132]. Our study is the first one to analyze muscles mass change and myonuclear distribution in vivo in large number of genes during Drosophila metamorphosis. By using time lapse image analysis and FMAj tool, we were able to characterize transient phenotypes and differentiate gene perturbations based on following criteria;

(1) whether they promoted or inhibited cell death,

(2) which muscles were affected,

(3) the developmental period of phenotypic abnormalities

(4) the morphological transformations during muscle destruction.

(5) the myonuclear distribution

Table 7 shows the comparison between the findings of previous studies and results of our analysis for respective genes during Drosophila metamorphosis.

\begin{tabular}{|l|l|l|}
\hline Gene & Previous studies & New phenotypes \\
\hline Atg5 & $\begin{array}{l}\text { During salivary gland degradation } \\
\text { caused by rise in ecdyson, an increase in } \\
\text { transcription of Atg5 was observed } \\
{[133],[134]}\end{array}$ & $\begin{array}{l}\text { 1. Increase in thickness of } \\
\text { muscle observed in DIOMs } \\
\text { due to knockdown of gene. } \\
\text { Selective targeting of the } \\
\text { DIOMs observed; whereas } \\
\text { other muscles i.e. IFM, DIOM } \\
\text { were unaffected. } \\
\text { 2. Atg } 5 \text { does not promote } \\
\text { atrophy throughout } \\
\text { metamorphosis. Hypertrophy } \\
\text { in DIOMs is observed after } \\
\text { head eversion. } \\
3 . \quad \text { Abnormality in anti- } \\
\text { polar/polar migration and } \\
\text { lateral distribution of nuclei }\end{array}$ \\
\hline
\end{tabular}




\begin{tabular}{|c|c|c|}
\hline & & $\begin{array}{l}\text { was observed. However, they } \\
\text { could be dependent on the } \\
\text { change in shape of muscle. }\end{array}$ \\
\hline $\operatorname{Atg} 9$ & $\begin{array}{l}\text { Role of Atg9 in autophagy under nutrient } \\
\text { deprivation has been studied in midgut } \\
\text { [135, p. 9] and fat body cells [136, p. 18] } \\
\text { in Drosophila }\end{array}$ & $\begin{array}{l}1 \& \text { 2. Same as Atg } 5 \\
\text { 3. Higher clustering of nuclei } \\
\text { toward muscle cell center was } \\
\text { observed due to prolonged } \\
\text { anti-polar migration. } \\
\text { 4. Larger lateral displacement } \\
\text { of nuclei along width of } \\
\text { muscle was observed in Atg9 } \\
\text { RNAi. However, it could be } \\
\text { the result of increase in muscle } \\
\text { width. }\end{array}$ \\
\hline $\operatorname{Atg} 12$ & $\begin{array}{l}\text { Knockdown of Atg12 resulted in } \\
\text { incomplete degradation of the larval } \\
\text { salivary glands [137] }\end{array}$ & $\begin{array}{l}\text { 1 \& 2. Same as Atg5 } \\
\text { 3. The effect of Atg } 12 \\
\text { knockdown in muscle width } \\
\text { change is less compared to } \\
\text { Atg5, Atg9 and Atg18. Despite } \\
\text { increase in DIOM width, no } \\
\text { significant increase in the area } \\
\text { of the muscle cells was } \\
\text { observed. } \\
\text { 4. Same as (3) of Atg5 }\end{array}$ \\
\hline $\operatorname{Atg} 18$ & $\begin{array}{l}\text { - Knockdown of Atg18 resulted in } \\
\text { incomplete degradation of the larval } \\
\text { salivary glands [137] and delayed } \\
\text { midgut removal [138]. } \\
\text { - Interaction between Atg9 and Atgl8 in } \\
\text { autophagy has been studied during }\end{array}$ & $\begin{array}{l}1 \& 2 . \text { Same as } \operatorname{Atg} 5 \\
\text { 3. Same as } \operatorname{Atg} 9 \\
\text { 4. Same as } \operatorname{Atg} 9\end{array}$ \\
\hline
\end{tabular}




\begin{tabular}{|c|c|c|}
\hline & $\begin{array}{l}\text { starvation in Drosophila fat bodies [136, } \\
\text { p. 18]. }\end{array}$ & \\
\hline Atg17 & $\begin{array}{l}\text { Atg } 17 \text { promotes autophagy in an Atgl } \\
\text { dependent manner in salivary gland, } \\
\text { midgut and fat bodies }[139, \text { p. } 17],[140]\end{array}$ & $\begin{array}{l}\text { Atg17 knockdown caused } \\
\text { hypertrophy, cell death and } \\
\text { abnormal morphology in } \\
\text { DIOMs. }\end{array}$ \\
\hline Grunge & $\begin{array}{l}\text { It has been shown that Grunge } \\
\text { overexpression contributed to cell death } \\
\text { in the nervous system [141] }\end{array}$ & $\begin{array}{l}\text { Grunge silencing causes } \\
\text { selective inhibition of DEOM } \\
\text { cell death and appearance of } \\
\text { large vacuoles in the persistent } \\
\text { DEOMs suggesting } \\
\text { that Grunge may play a role in } \\
\text { promoting muscle } \\
\text { degeneration. }\end{array}$ \\
\hline SNFIA & $\begin{array}{l}\text { Loss of } d A M P K \alpha \text { results in reduction of } \\
\text { thickness of midgut musculature [142] }\end{array}$ & $\begin{array}{l}\text { 1. AMPK } \alpha \text { RNAi leads to loss } \\
\text { of tubular morphology and } \\
\text { degeneration of DIOMs. } \\
\text { 2. Other muscle were not } \\
\text { affected by AMPK } \\
\text { knockdown. }\end{array}$ \\
\hline Cpl & $\begin{array}{l}\text { It has been shown that expression of } \\
C p 1 \text { is upregulated during salivary } \\
\text { gland cell death indicating its role in } \\
\text { autophagic cell death [133] }\end{array}$ & $\begin{array}{l}\text { 1. } C p 1 \text { acts as a suppressor of } \\
\text { cell death in DIOMs } \\
2 . \\
T O R^{T E D} \text { overexpression } \\
\text { and } R m 62 \mathrm{RNAi} \text {, cell death } \\
\text { due to } C p 1 \text { knockdown } \\
\text { occurred in a later stage of } \\
\text { metamorphosis. }\end{array}$ \\
\hline
\end{tabular}




\begin{tabular}{|l|l|l|}
\hline $\mid \begin{array}{l}3 . \quad \text { Anti-polar and polar } \\
\text { migration of nuclei was not } \\
\text { observed in } C p 1 \text { RNAi } \\
\text { indicating that } C p 1 \text { is required } \\
\text { for myonuclear migration. }\end{array}$ \\
\hline
\end{tabular}

Table 7. Comparison between the findings of previous studies and results of our analysis for respective genes during Drosophila metamorphosis

The results of phenotypic analysis of these genes using our screening protocol and custom muscle analysis algorithms has proven that Drosophila metamorphosis can be used as disease model to study muscles. Next, i'll discuss the results of both qualitative as well as quantitative phenotypic analysis in detail.

The RNAi line of each gene was crossed with the double marker reporter line (MHC-tauGFP/FM7-GFP; Mef2-Gal4, UAS-Histone 2Av-mKO/ TM6B Tb). In each experiment 20 progenies per cross were examined. The progeny of the crosses were classified on the basis of lethality stages i.e. prepupal, early pupal, late pupal and adult. The numbers recorded under lethality test columns were the count of the samples which survived during a particular developmental phase (Table 8). Next, we used macro zoom fluorescence microscope (Stereo microscope) to analyze the phenotype of the muscle. As the images from the stereo microscope were collected only at an interval of 6-12 hours, our capacity to examine progression of a phenotype was limited. Thus, the objective of this analysis was to qualitatively identify the type of phenotype observed in muscles. We used Chromator (Chro) RNAi as a control for genetic perturbation. Chro mutants did not show any morphological muscle abnormalities and the adults were fertile and capable of flying. We compared the morphology, degree of histolysis, spatial and temporal patterning of muscles in genetic crosses with the control. We also examined the spatial distribution of myonuclei. Figure 41 shows the muscle development in a control sample. In the case of absence of defective muscle phenotype, we concluded that either RNAi could not achieve partial/complete gene silencing or the gene is not actively involved in muscle cell death during metamorphosis. The results of lethality test and muscle phenotype analysis are shown in Table 8 . The gene without ** 
in Table 8 were studied previously in our lab. First, we will briefly discuss the findings of the screens done in the previous study and then show the results of further analysis done in the current work.

Prepupal lethality was observed in Ecr-IR (Inverted repeat), Mtor-IR, Tor-IR, Tor$O E$ (Overexpression) and Tor $^{T e d}-O E$ lines in the previous study (Figure 42). The role of Ecr and Mtor in muscle remodeling was not confirmed because of high penetration rate of lethality (100\% in Ecr, 95\% in Mtor). Overexpression of Tor and Tor ${ }^{\text {Ted }}$ resulted in cell death whereas silencing of Tor resulted in atrophy of persistent muscles. Cell death of persistent muscles was also observed in $C p 1$ and $R M 62$ mutant. Atrophy of muscles was observed in Rheb, a positive regulator of Tor. Whereas silencing of Tscl, $T s c 2$ and autophagy related genes resulted in hypertrophy of the muscles. The only gene which affected the IFMs development was yorkie. The knockdown of yorkie resulted in cell death and atrophy of IFMs. Abnormal distribution of nuclei was associated with silencing of the autophagy related genes and $C p l$. The results discussed above were found in the previous study [114]. In our current work, we found two genes with interesting muscle phenotypes. In Grunge mutants, inhibition of DEOMs histolysis was observed. On the other hand, knockdown of SNF1A resulted in complete destruction of DIOMs.

In order to further analyze the observed phenotypes, the lines which did not show prepupal lethality were imaged using confocal microscopy (Shown in red in Table 8). The advantage of confocal microscopy is that we can collect high resolution images at shorter temporal intervals in an automated manner. This reduced the manual intervention during image acquisition and thereby the related errors. Time lapse images of pupae were collected at an interval of $30 \mathrm{~min}$ at $23^{\circ} \mathrm{C}$. A window of 30 minute was small enough to ensure that we do not miss any phenotypic changes in muscles. Only three lines i.e. Grunge, $S N F 1 A$ and $A \operatorname{tg} 17$ were imaged at $25^{\circ} \mathrm{C}$. At $23^{\circ} \mathrm{C}$, Drosophila pupa took approximately $+100 \mathrm{~h}$ to develop into adult whereas at $25^{\circ} \mathrm{C}$ the development time reduced to approximately $+80 \mathrm{~h}$.

Next, we extracted the quantitative features for characterizing different phenotypes using the FMAj tool and measured their statistical significance. Before calculating the morphological features of muscles, they were segmented using the three methods mentioned in chapter 4 . Muscle width, area and length were used to quantify the muscle 
mass change. Extent and elongation were used to quantify the changes in the shape of the muscle. In every morphological analysis we have used DIOM1 [143] from abdominal segment 3 for comparison. Nuclear spatial patterns were calculated using the pipeline explained in chapter 5. The results of quantitative phenotypic analysis are discussed in detail in next section. 


\begin{tabular}{|c|c|c|c|c|c|c|c|c|c|c|c|c|c|c|c|c|c|}
\hline 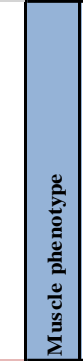 & & 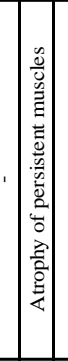 & 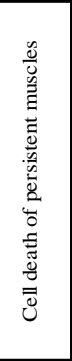 & 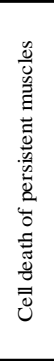 & 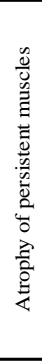 & 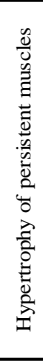 & 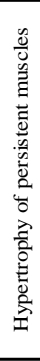 & 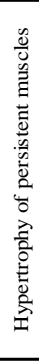 & 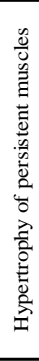 & 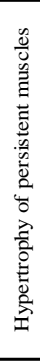 & 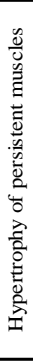 & 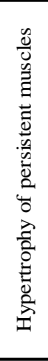 & 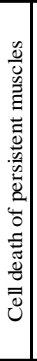 & 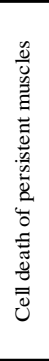 & 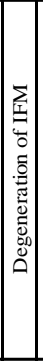 & 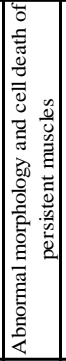 & 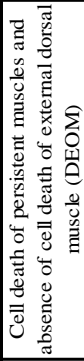 \\
\hline 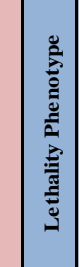 & 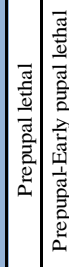 & 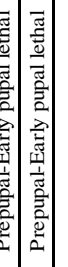 & 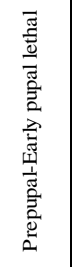 & 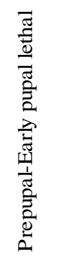 & & & & & & & & & & & & & \\
\hline$\frac{\bar{z}}{\frac{\bar{z}}{4}}$ & 00 & $\circ A$ & 0 & $a$ & 2 & $\stackrel{2}{2}$ & $\simeq$ & ¿ิ & ¿ิ & $=$ & 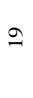 & तి & ते & $\stackrel{\varrho}{\circ}$ & $\simeq$ & $\stackrel{\infty}{=}$ & 2 \\
\hline 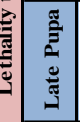 & 0 - - & $-1 \mathrm{~N}$ & 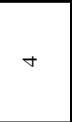 & $\simeq$ & तి & तి & $\underline{0}$ & ¿ & त & $=$ & 2 & ¿ి & त) & तิ & $\simeq$ & $\stackrel{\infty}{\simeq}$ & สิ \\
\hline 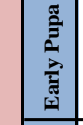 & নి & 교 & $\stackrel{\infty}{=}$ & तి & సి & సి & ๙ & ¿ิ & तి & तి & 2 & శి & ते & तิ & त) & สิ & สิ \\
\hline 竎 & तิ ন্ & 워 & $\stackrel{\infty}{=}$ & तิ & సి & ন & तి & तิ & तి & तి & तิ & त) & तิ & ते & ते & ì & तิ \\
\hline 晜 & 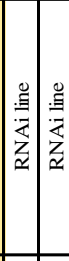 & 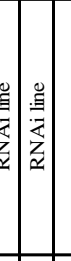 & 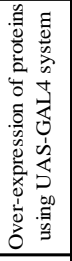 & 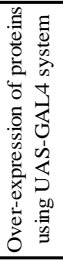 & 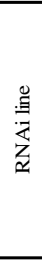 & 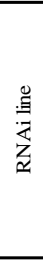 & 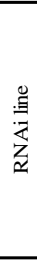 & 莺 & $\begin{array}{l}\text { 盿 } \\
z \\
z\end{array}$ & 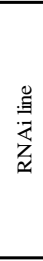 & & 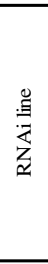 & 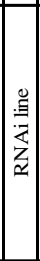 & 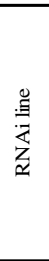 & 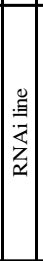 & 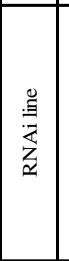 & 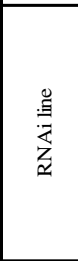 \\
\hline 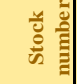 & 啇 & 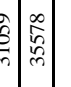 & $\frac{1}{2}$ & $\stackrel{m}{2}$ & $\begin{array}{l}\text { : } \\
\stackrel{0}{\text { m. }}\end{array}$ & $\underset{m}{\stackrel{J}{*}}$ & $\begin{array}{l}\hat{m} \\
\hat{f} \\
\text { nd }\end{array}$ & 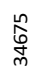 & 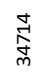 & $\begin{array}{l}\text { ब. } \\
\text { 岕 }\end{array}$ & 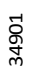 & $\frac{\infty}{0}$ & 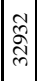 & 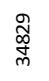 & $\left|\begin{array}{|c}\hat{\delta} \\
\bar{m}\end{array}\right|$ & $\stackrel{\tilde{m}}{\stackrel{m}{m}}$ & $\begin{array}{l}\overline{0} \\
\text { ले }\end{array}$ \\
\hline 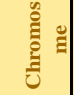 & $m$ & $n \mathrm{~m}$ & $m$ & $N$ & $m$ & $m$ & $m$ & $m$ & $m$ & $m$ & $m$ & $m$ & $m$ & $m$ & $m$ & $m$ & $m$ \\
\hline ญँّ & 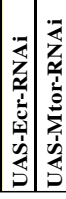 & 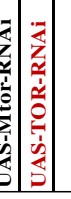 & 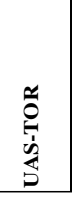 & 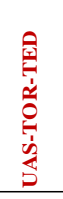 & 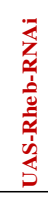 & 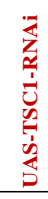 & 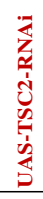 & 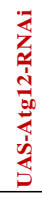 & 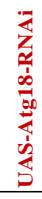 & 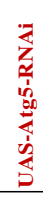 & 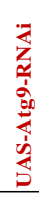 & 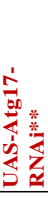 & 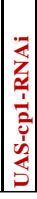 & 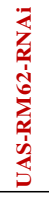 & 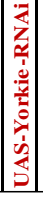 & 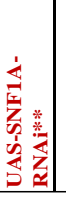 & 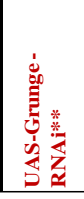 \\
\hline
\end{tabular}

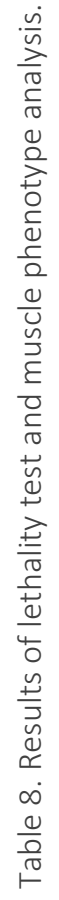


(a)

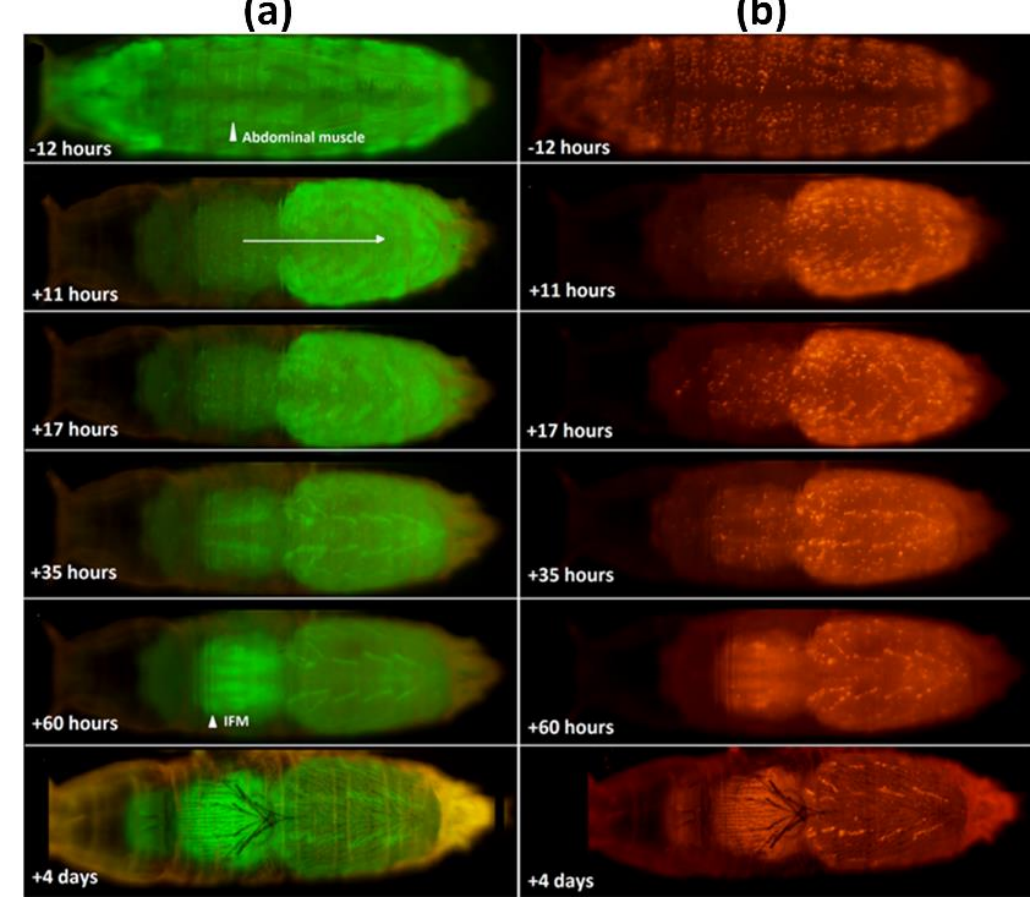

Figure 41. Muscle development during the different stages of metamorphosis.

(a) Green channel visualizes the muscle cells. (b) Red channel visualizes the muscle cell nuclei. The onset of Head eversion or prepupal to pupal transition (PPT) is assigned the time stamp 0 hours on the time scale.

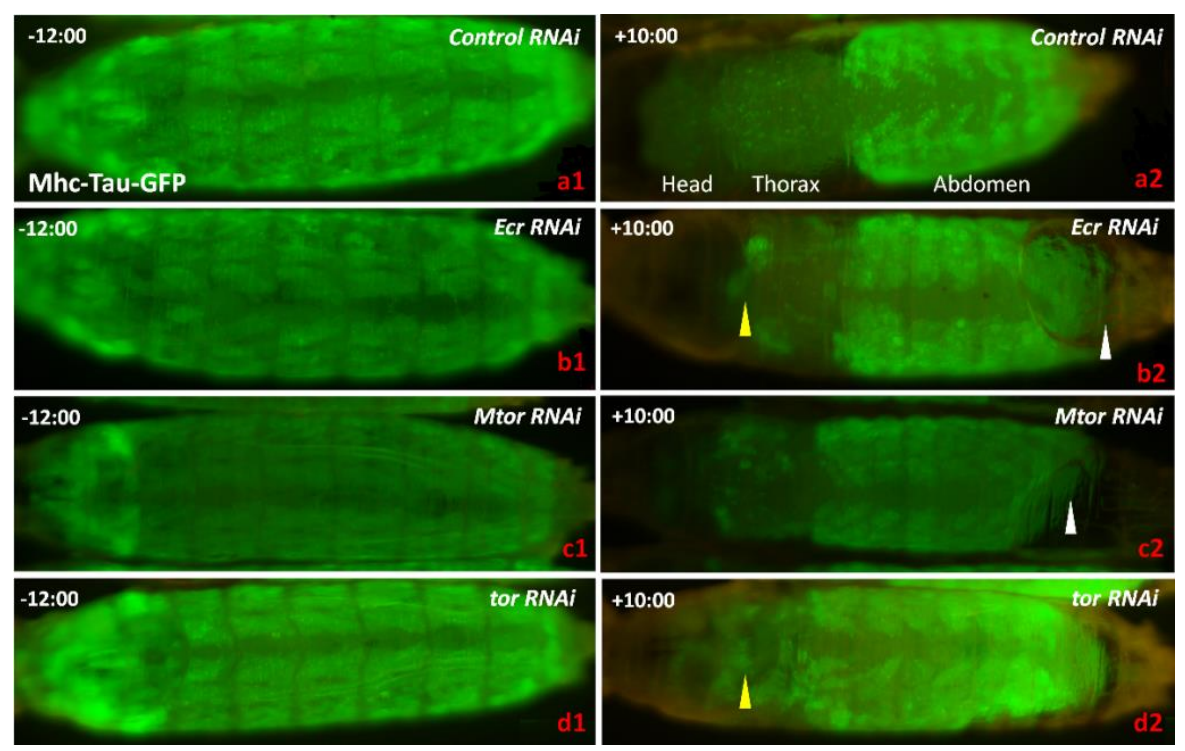

Figure 42. Defective development in prepupal lethal mutants.

White arrowhead shows the incomplete separation of prepupa from cuticle. Yellow arrowhead shows the incomplete development of the head and thorax. 


\subsubsection{Morphological analysis of muscle cells}

\section{PI3K signaling pathway regulates the persistent muscle mass change during metamorphosis}

We were able to quantitatively confirm the results from previous screens that Tor, Rheb, Tsc1 and Tsc2 regulate mass of persistent muscles during metamorphosis. Knockdown of Tor and Rheb caused decrease in the width of the persistent muscles (DIOM) during metamorphosis (Figure 43 column b \& c). The width and area of muscles was significantly lower than the control throughout the metamorphosis (Figure 43 top \& middle graph). We also found that Tor silencing resulted in reduced length of muscle cells as compared to control (Figure 43 bottom graph). Whereas Rheb silencing did not show significant difference in muscle length except around $30 \mathrm{~h}$ and $70 \mathrm{~h}$ (Figure 43 bottom graph). As compared to Rheb, silencing of negative regulator of Tor i.e. $T s c 1$ and $T s c 2$ resulted in hypertrophy of persistent muscles during metamorphosis (Figure 44 column b \& c). Both the area and width of muscles was significantly larger in $T s c 2$ mutants than control (Figure 44 top \& middle graph). The increase in muscle size due to silencing of $T s c 1$ is not as high as $T s c 2$. We observed significant increase in the muscle cell width and area between $+15 \mathrm{~h}$ and $+70 \mathrm{~h}$. We did not observe any significant difference in the length of muscle cells in Tsc1 and Tsc2 mutants (Figure 44 bottom graph).

\section{Atg 5, Atg 9, Atg 12 and Atg 18 promote atrophy in persistent muscles}

Quantitative analysis confirmed that silencing of $\operatorname{Atg} 5, \operatorname{Atg} 9, \operatorname{Atg} 12$ and $\operatorname{Atg} 18$ resulted in significant increase in the size of persistent muscles as described in the previous screen. However, the degree of hypertrophy in muscles varied between these genes. Before $+25 \mathrm{~h}$, there was no difference in the area of Atg5, Atg9, Atg12 and Atg18 mutants and control (Figure 45). After $+25 \mathrm{~h}$, the width of muscles in these four Atg mutants was significantly higher than the control (Figure 45 top graph). Atg5, Atg9, Atg18 mutants showed similar trends in change of the muscle width, area and length during development. However in Atg12 mutants, the increase in the width of muscles was compensated by the reduced length resulting in no significant increase in the area of the muscle cells (Figure 45 middle \& bottom graph). This time series analysis 
showed that these Atg genes do not promote atrophy throughout metamorphosis and selectively target the DIOMs.

Atg mutants also showed central bulging in the muscle cells. The low values of elongation in $\operatorname{Atg} 9$ and $\operatorname{Atg} 18$ mutants after $+35 \mathrm{~h}$ showed that the muscle cells were larger in the middle as compared to the poles (Figure 46a, b). Whereas, the values of elongation-S, were significantly low for $\operatorname{Atg} 9$ and $\operatorname{Atg} 18$ after $+27 \mathrm{~h}$ approximately (Figure 46c, d). This indicates that between $+27 \mathrm{~h}$ and $+35 \mathrm{~h}$ the muscle cell were bent in structure which resulted in lower value of elongation-S than elongation. We also compared extent and extent-S to understand the shape changes in muscles. After +50 $57 \mathrm{~h}$, extent of muscles in Atg9 and Atg 18 mutants was higher than control. Since extent does not consider the orientation of the muscle cell; we can only conclude that higher extent of the two Atgs is the result of their increased rectangular-ness as compared to control (Figure 47 a \& b). However, extent of straightened muscle (Extent-S) cell can be used to measure the variation in width of muscles along medial axis. The graph in figure 47 (c \& d) shows that Extent-S is not significantly different for the two Atg mutants and control. The DIOMs in Atg9 and Atg18 are wider near the center of muscle cell whereas in control the muscles are wider near poles. Due to this phenotype, extent$\mathrm{S}$ in the two Atg mutants and control are similar.

As compared to the Atgs mentioned above, knockdown of Atg17 resulted in multiple muscle phenotypes. We analyzed the DIOM1 of every abdominal segment in Atg 17 mutant and found that 15\% were hypertrophied, 33\% died during pupal stage and $51 \%$ formed a circular structure. The role of Atg17 in activation of autophagy could not be concluded from these results.

\section{SNF1A silencing cause cell death of persistent muscles}

In our study, SNF1A silencing resulted in cell death of all the DEOMs (Figure 48). After the histolysis of DEOMs, the DIOM continued to break down until $+25 \mathrm{~h}$. After $+25 \mathrm{~h}$, the muscle cells formed a circular structure and towards the end of metamorphosis they were destroyed completely. We compared the cell death phenotype between SNF1A mutants and the previously studied TOR ${ }^{T E D}-O E, C P 1-I R$ and RM62-IR lines (Figure 48). In both $S N F 1 A$ mutant and TOR ${ }^{T E D}$ overexpression, the histolysis of DIOMs started immediately after head eversion and all of them were destroyed by the end of metamorphosis. However, the circular structures that muscles formed during 
disintegration were much larger in SNF1A mutant as compared to TOR ${ }^{T E D}$ overexpression (Figure 48-b4 \& Figure 48-b3). Also, TOR ${ }^{T E D}$ overexpression delayed histolysis of DEOMs (Figure 49-b1). This phenotype was not observed in SNF1A. Cell death of DIOMs in CPI and RM62 mutant occurred later as compared to SNF1A mutant. Table 9 lists the time (hours after head eversion) at which DIOM1 started histolysing in CP1 and RM62 mutants. Unlike SNF1A, histolysis of DIOMs due to the knockdown of RM62 started after the histolysis of DEOMs was completed i.e. at 10 - 16 hours. Silencing of SNF1A, RM62 and TOR ${ }^{T E D}$ overexpression, resulted in generation of large amount of muscle debris during histolysis of DIOMs and the eventual destruction of every DIOM. Unlike these three genes, $C p 1$ knockdown caused cell death of only a subset of DIOMs at different time points spanning between $+30-80 \mathrm{~h}$. Also, we did not observe large amount of muscle debris during cell death of DIOMs in $C p 1$ mutants. These results showed that the four genes (SNF1A, Tor, RM62 and CP1) participate in muscle remodeling at different stages of metamorphosis. SNF1A did not affect the histolysis of DEOMs. Whereas it triggered the histolysis of DIOMs after head eversion; this indicates that SNF1A might not be participating in muscle remodeling before head eversion.

\begin{tabular}{|c|c|c|c|c|c|c|c|c|c|c|}
\hline Cp1 & \multicolumn{2}{|c|}{ Segment 1 } & \multicolumn{2}{c|}{ Segment 2 } & \multicolumn{2}{c|}{ Segment 3 } & \multicolumn{2}{c|}{ Segment 4 } & \multicolumn{2}{c|}{ Segment 5 } \\
\cline { 2 - 11 } & $\begin{array}{c}\text { DIOM 1 } \\
\text { (Right) }\end{array}$ & $\begin{array}{c}\text { DIOM 1 } \\
\text { (Left) }\end{array}$ & $\begin{array}{c}\text { DIOM 1 } \\
\text { (Right) }\end{array}$ & $\begin{array}{c}\text { DIOM 1 } \\
\text { (Left) }\end{array}$ & $\begin{array}{c}\text { DIOM 1 } \\
\text { (Right) }\end{array}$ & $\begin{array}{c}\text { DIOM 1 } \\
\text { (Left) }\end{array}$ & $\begin{array}{c}\text { DIOM 1 } \\
\text { (Right) }\end{array}$ & $\begin{array}{c}\text { DIOM 1 } \\
\text { (Left) }\end{array}$ & $\begin{array}{c}\text { DIOM 1 } \\
\text { (Right) }\end{array}$ & $\begin{array}{c}\text { DIOM 1 } \\
\text { (Left) }\end{array}$ \\
\hline Sample 1 & - & 50 & 84.5 & - & 84.5 & - & 84.5 & - & 58.5 & - \\
\hline Sample 2 & 63 & 63 & 90 & 90 & - & - & - & - & - & - \\
\hline Sample 3 & 37 & 52 & 52 & 52 & 62 & - & - & 69 & 52 & - \\
\hline Sample 4 & 45 & - & 55.5 & 55.5 & 64.5 & - & - & - & 40 & 55.5 \\
\hline Sample 5 & 42.5 & 42.5 & 42.5 & 42.5 & 62 & - & 62 & 62 & - & - \\
\hline Sample 6 & - & 31 & 50 & 92.5 & 92.5 & 75 & 50 & 75 & 50 & 75 \\
\hline Sample 7 & 58 & - & 58 & 70 & - & - & - & - & - & - \\
\hline Sample 8 & 38.5 & 38.5 & - & 68 & 95 & - & - & - & - & 46.5 \\
\hline Sample 9 & 34 & 53 & 90.5 & 69 & 53 & - & 69 & - & 53 & - \\
\hline Sample 10 & 40.5 & 51.5 & 51.5 & 73 & 59.5 & 59.5 & 51.5 & 40.5 & 51.5 & 51.5 \\
\hline
\end{tabular}

\begin{tabular}{|c|c|}
\hline RM62 & All DIOMs \\
\hline Sample 1 & 10 \\
\hline Sample 2 & 13 \\
\hline Sample 3 & 12 \\
\hline Sample 4 & 16 \\
\hline Sample 5 & 13 \\
\hline Sample 6 & 15 \\
\hline
\end{tabular}


Table 9. Time (Hours after head eversion) at which DIOM1 starts histolysing in different segments of the abdomen for Cp1 and RM62 mutants. The yellow cell indicates the group of muscle cells in a sample which die at the same time in Cp1 mutant.

\section{Grunge silencing causes selective inhibition of DEOM cell death}

In our study, silencing of Grunge ( $\mathrm{gug}$ ) led to inhibition of DEOM histolysis in abdominal segment 1 and 2 (Figure 50 b2 - b4). We observed that in gug mutants, DEOM1 in both segment 1 and 2 did not undergo histolysis. Whereas, 90\% of DEOM2 survived in segment 1 as compared to 8\% in segment 2. Neither DEOM1 nor DEOM2 escaped histolysis in the segments three to five. We confirmed that the DEOMs were not missing before head eversion. Gug silencing also resulted in the appearance of large vacuoles in the persistent DEOMs. High resolution 3D images (20x) of DEOMs were taken to visualize the vacuoles (Figure $50 \mathrm{c} 1, \mathrm{~d} 1$ ). Orthogonal view of the 3D stacks were generated using Fiji [88] to show the vacuoles inside DEOMs (Figure 50 c2, d2).

\subsubsection{Nuclear spatial pattern analysis results}

We used the nuclei spatial pattern analysis pipeline to quantify the effects of genetic perturbation on nuclei arrangement in persistent muscles. In this study, we compared the effects of loss of $C p l$ and Atgs on nuclear distribution with the control using our nuclear spatial pattern analysis pipeline. We have also compared the properties of nuclear tracks between different genotypes like start and end time of track, length of track, nucleus speed etc (Table 10). It shows that external nuclei display high speed and large movements compared to internal nuclei irrespective of genotype.

\section{Cp1 participates in the anti-polar/polar migration of nuclei}

Quantitative nuclear pattern analysis confirmed that the knockdown of $C p 1$ affected anti-polar nuclear migration in early pupation (Figure 51c, d). In control muscles, anti-polar migration reduced the expanse of nuclei along the medial axis $\left(L_{n}\right)$ by $\sim 97.5 \mu \mathrm{m}$ between $+28.9 \mathrm{~h}(205.8 \mu \mathrm{m})$ and $+46.6 \mathrm{~h}(108.4 \mu \mathrm{m})($ Table 11 , Figure $51 \mathrm{a}, \mathrm{b})$. By contrast, $C p 1$ silencing resulted in reduction of $L_{n}$ by $\sim 58.6 \mu \mathrm{m}$ between $+23.5 \mathrm{~h}(209.8 \mu \mathrm{m})$ and $+54.1 \mathrm{~h}(151.3 \mu \mathrm{m})$ (Figure 55a). Unlike control, where nuclear polar migration increased the value of $L_{n}$ by $\sim 82.6 \mu \mathrm{m}$ between $+46.6(108.4 \mu \mathrm{m}) \mathrm{h}$ and $+72.1 \mathrm{~h}(190.9 \mu \mathrm{m})$; Cpl RNAi did not show polar migration. Due to decreased 
anti-polar migration in Cpl RNAi, the values of $L_{n}$ are significantly different for control and $C p 1$ RNAi between $+42 \mathrm{~h}$ and $+52 \mathrm{~h}$ (Figure 52a, 52b).

The normalized longitudinal nuclear spread $\left(N M_{l o n}\right)$, indicated that the reduction in expanse of nuclei along medial axis of muscle $\left(L_{n}\right)$ in $C p l$ RNAi is due to shortening of muscle cells. This phenomenon was confirmed by very small change in values of $N M_{l o n}$ for $C p 1$ RNAi between $+32.5 \mathrm{~h}$ and $+60 \mathrm{~h}$ (Figure 52c).Between $+32.5 \mathrm{~h}$ and $+45 \mathrm{~h}, N M_{\text {lon }}$ values changed only by $0.2(0.82 \pm 0.07-0.84 \pm 0.05)$ for $C p 1$ RNAi as compared to $0.27(0.83 \pm 0.05-0.55 \pm 0.13)$ for control. Similarly, between $+45 \mathrm{~h}$ and +60 h, $N M_{\text {lon }}$ values changed only by $0.2(0.84 \pm 0.05-0.85 \pm 0.02)$ for $C p 1$ RNAi as compared to $0.28(0.55 \pm 0.13-0.83 \pm 0.03)$ for control. The significantly high values of $N M_{l o n}$ for $C p l$ RNAi as compared to control between $+40 \mathrm{~h}$ and $+57 \mathrm{~h}$ indicate that the knockdown of $C p 1$ resulted in absence of anti-polar migration (Figure 52d).

Silencing of $\operatorname{Atg} 9$ and $\operatorname{Atg} 18$ increases the anti-polar migration and decreases the polar migration of nuclei

RNAi of Atg5, Atg9 and Atg18 affected myonuclear migration. While knockdown of all Atgs RNAi did not block anti-polar and polar nuclear migration (Figure 45), the silencing of Atg9 and Atg 18 resulted in prolonged anti-polar migration of nuclei $(7.5 \mathrm{~h}$ (median) delay for Atg9 RNAi and $4 \mathrm{~h}$ (median) delay for Atg18) and larger anti-polar movement of nuclei (between $+27.4 \mathrm{~h}$ and $+53 \mathrm{~h}$ for $\operatorname{Atg} 9$ and between $+22.4 \mathrm{~h}$ and $+48.1 \mathrm{~h}$ for $\mathrm{Atg} 18$ ) as compared to control (between $+28.9 \mathrm{~h}$ and $+46.6 \mathrm{~h}$ ) (Figure 53a, b). The reduction in value of $L_{n}$ during anti-polar migration in $\operatorname{Atg} 5, \operatorname{Atg} 9, \operatorname{Atg} 18$ and control was $\sim 130.8 \mu \mathrm{m}, \sim 133.9 \mu \mathrm{m}, \sim 164.4 \mu \mathrm{m}$ and $\sim 97.5 \mu \mathrm{m}$; this indicates that nuclei are farther from poles in Atg5, Atg9 and Atg18 as compared to control (Figure 55a, Table 11). Knockdown of Atg9 and Atg18 also reduced the polar migration of nuclei (Between $+55 \mathrm{~h}$ and $+72.5 \mathrm{~h}$ for Atg9 and between $+50 \mathrm{~h}$ and $+72.5 \mathrm{~h}$ for Atg18), resulting in larger distances between pole and nearest nucleus (Figure 53a, b). The value of $L_{n}$ at the end of polar migration in Atg9, Atg18 and control was $\sim 141.6 \mu \mathrm{m}$, $\sim 162.4 \mu \mathrm{m}$ and $\sim 190.9 \mu \mathrm{m}$ approximately; this indicates reduced polar migration in Atg9 and Atg18 (Table 11). However, it is important to note that in spite of lower longitudinal nuclear spread in Atgs, there is no significant difference between the changes in longitudinal nuclear spread (between start and end of polar migration) in Atgs and control as shown in Figure 55b.

The significant difference in $N M_{\text {lon }}$ between the control and $\operatorname{Atg} 9$ and Atg18 RNAi 
after $+50 \mathrm{~h}$ and $+46 \mathrm{~h}$ respectively proved that the polar/anti-polar migration of nuclei in Atg9 RNAi and Atg18 RNAi is not affected by muscle contraction/elongation

(Figure 53c, d). The effects of silencing Atg5 and Atg 12 on nuclear migration are not as prominent as $\operatorname{Atg} 9$ and $\operatorname{Atg} 18$ silencing. In both $\operatorname{Atg} 5$ and $\operatorname{Atg} 12$, the values of $L_{n}$ is significantly less than control at the end of polar migration $(\sim 169 \mu \mathrm{m}$ at $+72.1 \mathrm{~h}$ for Atg $5, \sim 177.1 \mu \mathrm{m}$ at $+72.2 \mathrm{~h}$ for Atg12 and $\sim 190.9 \mu \mathrm{m}$ for control), although not as low as Atg9 and Atg18; this indicates reduced polar migration (Table 11). Also, there was no significant difference in the values of $N M_{l o n}$ in Atg5 and Atg12 RNAi during the polar migration. These findings indicate that the decreased polar migration caused by silencing of Atg5 and Atg 12 could be due to contraction of muscle cells.

\section{Lateral myonuclear displacement Atg knockdowns correlates with increased diameter of muscles.}

As previously reported [14], loss of autophagy resulted in nuclei being arranged in two rows in late metamorphosis as compared to single row formation in control muscles. We used lateral nuclear spread $\left(M_{l a t}\right)$ and normalized lateral nuclear spread $\left(N M_{\text {lat }}\right)$ to quantify the lateral movement of nuclei perpendicular to the medial axis of muscles. In Atg9 and Atg 18 RNAi, the value of $M_{\text {lat }}$ was significantly higher in controls after $+53 \mathrm{~h}$ and $+50 \mathrm{~h}$, respectively; suggesting that nuclei moved away from the muscle's medial axis and arranged in a two-row formation (Figure 53e, f). Interestingly, we did not observe a similar trend in $N M_{\text {lat }}$ for $\operatorname{Atg} 9$ and $\operatorname{Atg} 18 \mathrm{RNAi}$ (Figure $53 \mathrm{~g}$ ). We found the values of $N M_{\text {lat }}$ were significantly lower for Atg9 RNAi compared to controls between $+50 \mathrm{~h}$ and $+70 \mathrm{~h}$ (Figure $53 \mathrm{~h}$ ). This indicates that between $+50 \mathrm{~h}$ to $+70 \mathrm{~h}$, the nuclei were located away from the muscle cell boundary as compared to control, even though they were arranged in two rows. However, after $+70 \mathrm{~h}$, values of $N M_{\text {lat }}$ were not significantly different between control and Atg9 RNAi. Whereas, in Atg18 RNAi, the value of $N M_{\text {lat }}$ were not significantly different from control throughout pupal development. Therefore, the lateral displacement of nuclei might be dependent on the change in muscle width in $\operatorname{Atg} 9$ and $\operatorname{Atg} 18$ RNAi. The increase in width of muscle cell due to loss of autophagy could be increasing the distance between nuclei resulting in two-row formation. In case of $\operatorname{Atg} 5$ and $\operatorname{Atg} 12$, the lateral displacement features were unable to statistically prove the two-row formation, despite being confirmed visually, indicating a lower penetration of the phenotype. 


\section{The anti-polar/polar migration and lateral displacement of nuclei affect the}

spatial density of nuclei in muscles

During early stages of pupal development, differences in spatial density of nuclei were observed between Atgs (Atg5, Atg9 \& Atg18) and control. In Atg5 RNAi, significant reduction in NSD was observed between $+25 \mathrm{~h}$ to $+42.5 \mathrm{~h}$ compared to control. This observation could be the result of larger anti-polar migration and smaller lateral displacement of nuclei with respect to width of muscle. Significant reduction in spatial density of nuclei was also observed in Atg9 (between $+30 \mathrm{~h}$ to $+40 \mathrm{~h}$ ) and Atg18 (between $+35 \mathrm{~h}$ to $+40 \mathrm{~h}$ ). We also observed that the spatial density of nuclei with respect to the muscle area in Atg9 and Atg 18 was significantly lower than the control between $+50 \mathrm{~h}$ to $+70 \mathrm{~h}$ and $+50 \mathrm{~h}$ to $+65 \mathrm{~h}$ respectively (Figure $54 \mathrm{a}, \mathrm{b}$ ). It indicates that the knockdown of both $\operatorname{Atg} 9$ and $\operatorname{Atg} 18$ increased the area devoid of nuclei in muscles. Since, the lateral displacement of nuclei increases between $+50 \mathrm{~h}$ to $+70 \mathrm{~h}$ in Atg9 and Atg18 RNAi; the reduced polar migration could be the reason for low spatial density of nuclei (NSD) with respect to the muscle area. As opposed to other Atgs, Atg12 knockdown did not affect the spatial density of nuclei with respect to the muscle area despite the two-row formation. Increased polar migration during later pupal development could be one of the reasons behind the control like nuclear spatial density in Atg5 and Atg12 RNAi as compared to Atg9 and Atg18 (Figure 53a, b). 


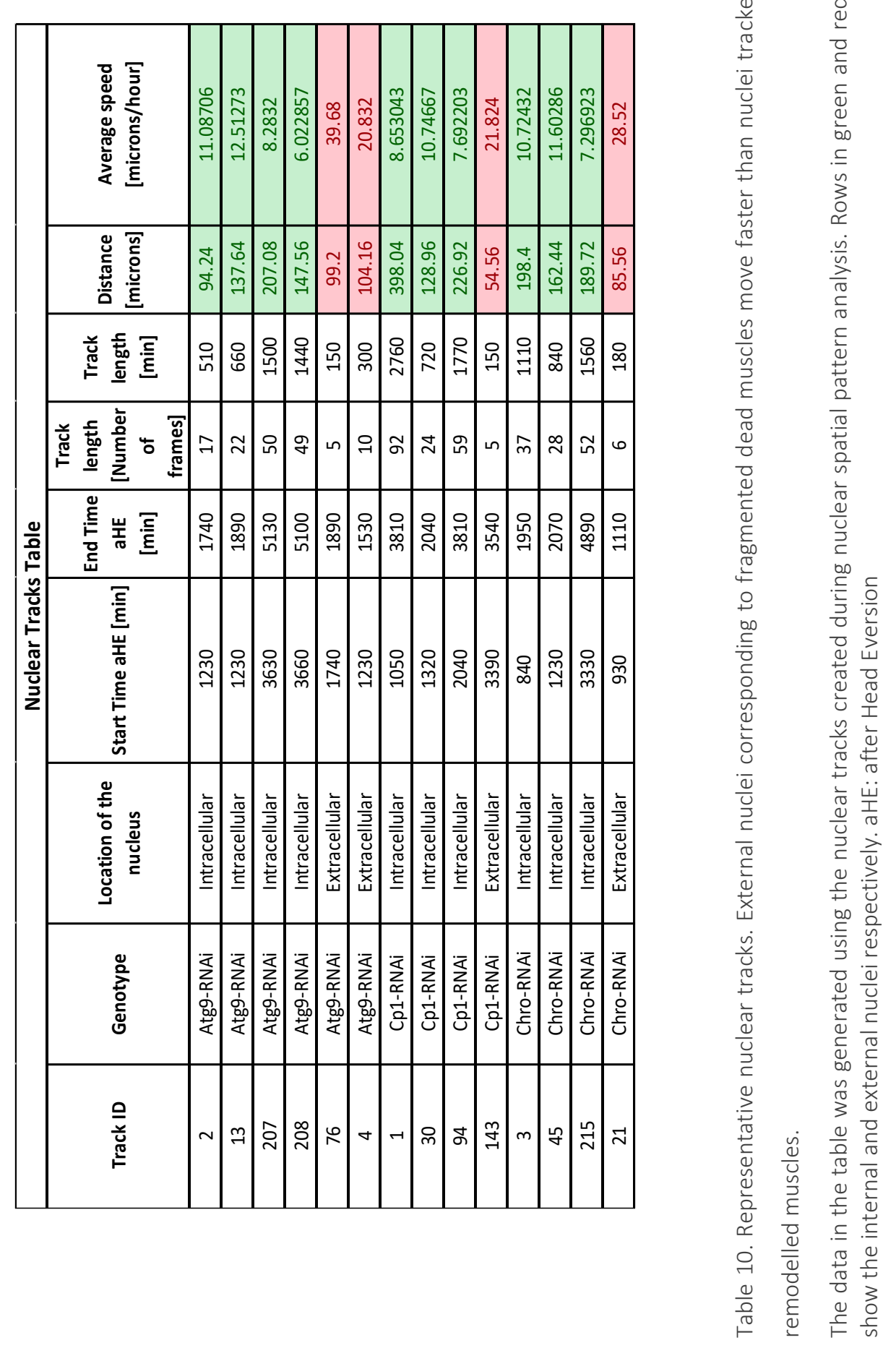




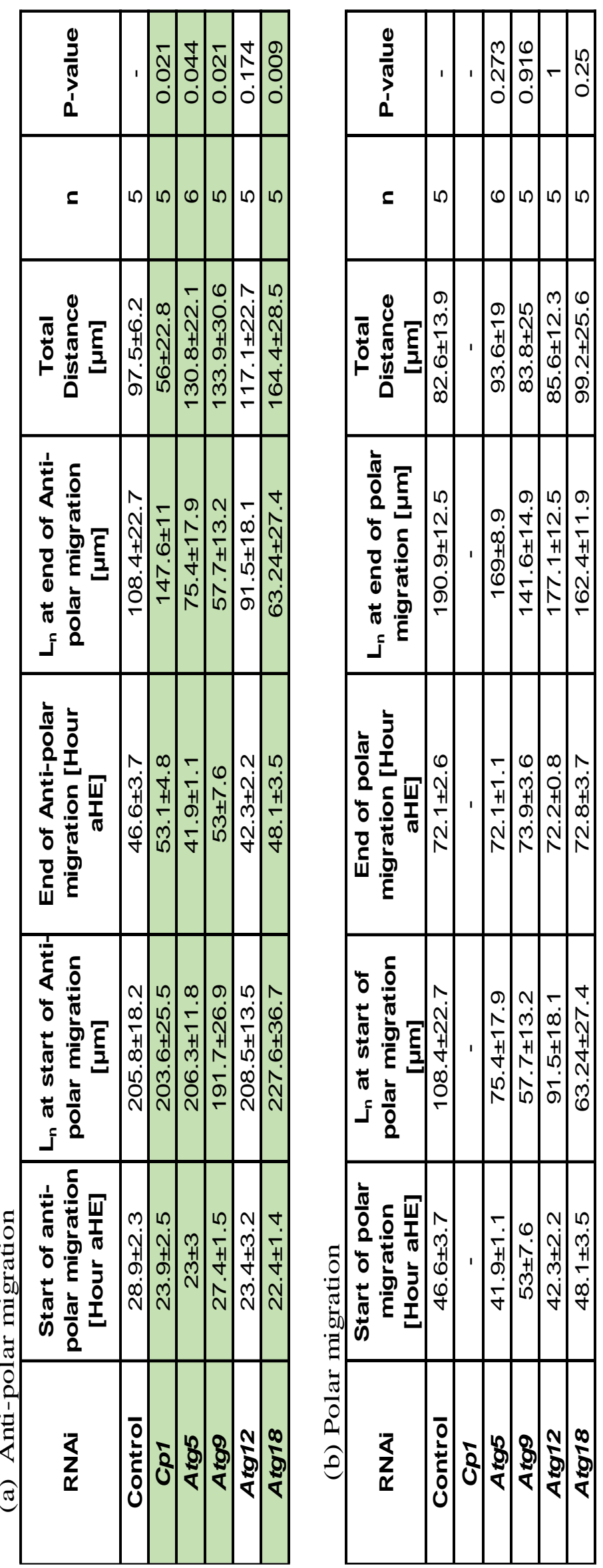

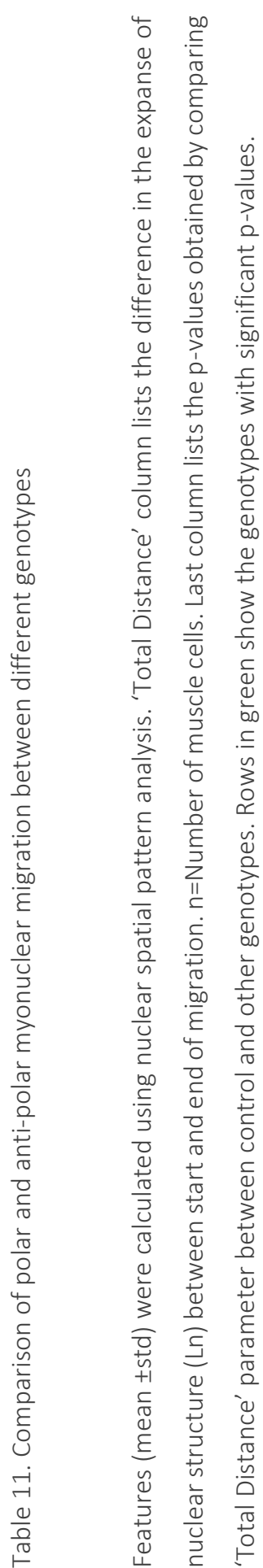



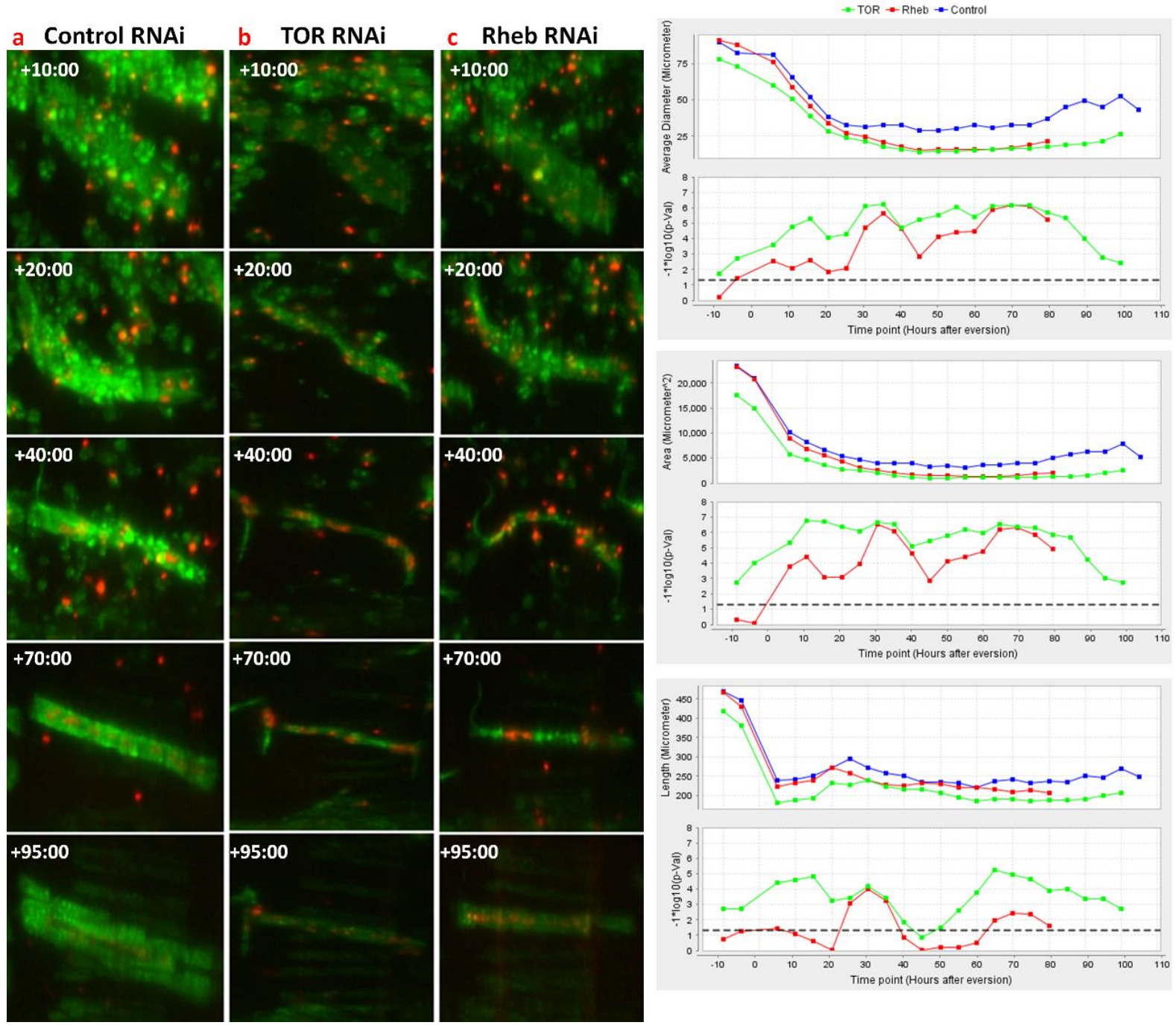

Figure 43. Knockdown of Tor and Rheb causes decrease in the width of the persistent muscles.

Left side of the figure shows the development of DIOM in control (a), Tor (b) and Rheb (c). The last three rows show the atrophied muscles in Tor and Rheb. Right side of the figure compares the median values of three morphological features of muscle cells expressing different RNAi constructs: width (Top), area (Middle) and length (Bottom). For each population, statistics were derived from 10-20 muscle cells. The horizontal dotted lines in the significance graphs (bottom panels) represent the p-value 0.05. 

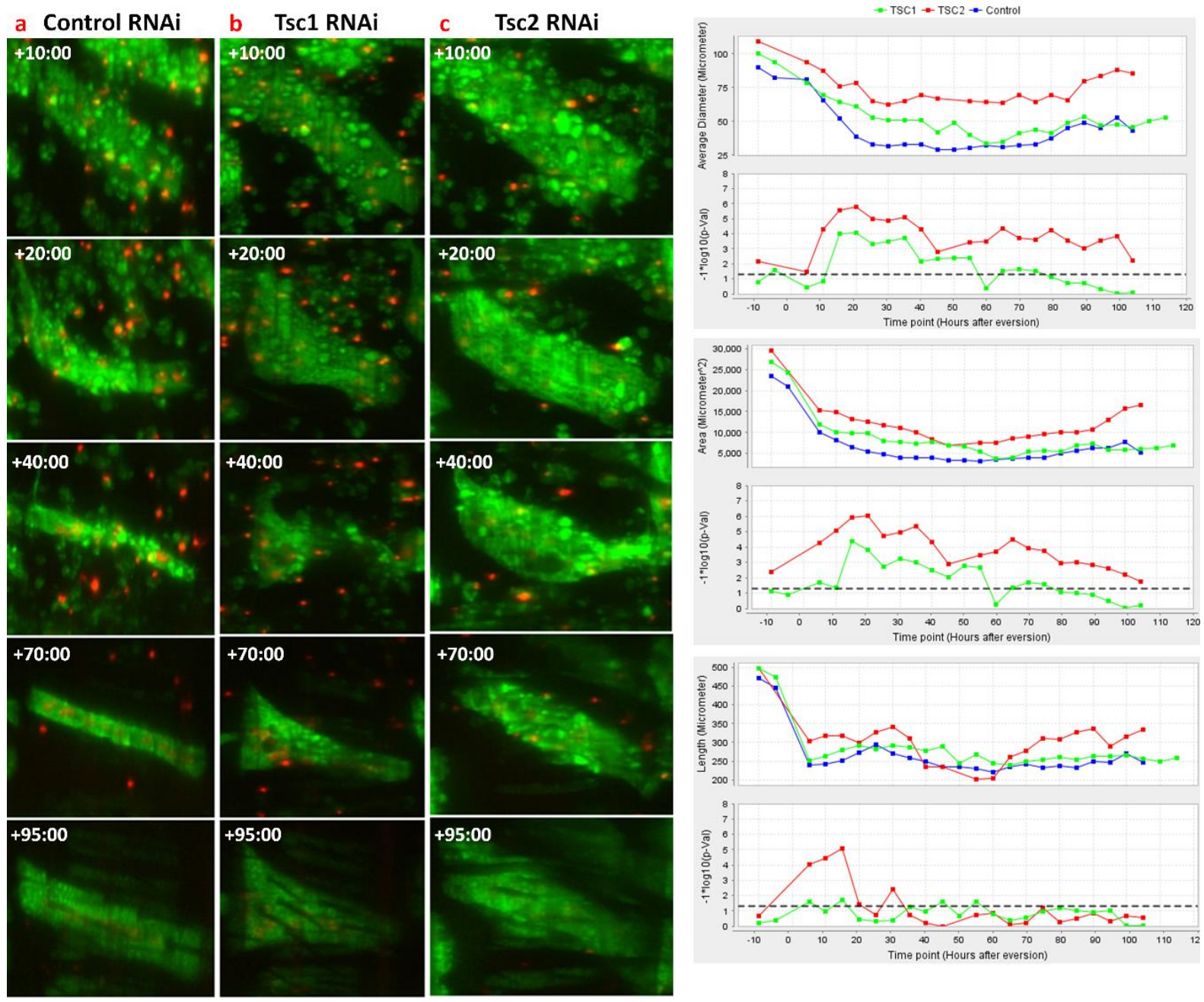

Figure 44. Silencing of $T s c 1$ and $T s c 2$, regulator of Tor, causes hypertrophy of persistent muscles.

Left side of the figure shows the development of DIOM in control (a), Tsc1 (b) and Tsc2 (c). The degree of hypertrophy in $T s c 2$ is higher than $T s c 1$. Right side of the figure compares the median values of three morphological features of muscle cells expressing different RNAi constructs: width (Top), area (Middle) and length (Bottom). For each population, statistics were derived from 10-20 muscle cells. The horizontal dotted lines in the significance graphs (bottom panels) represent the p-value 0.05. 

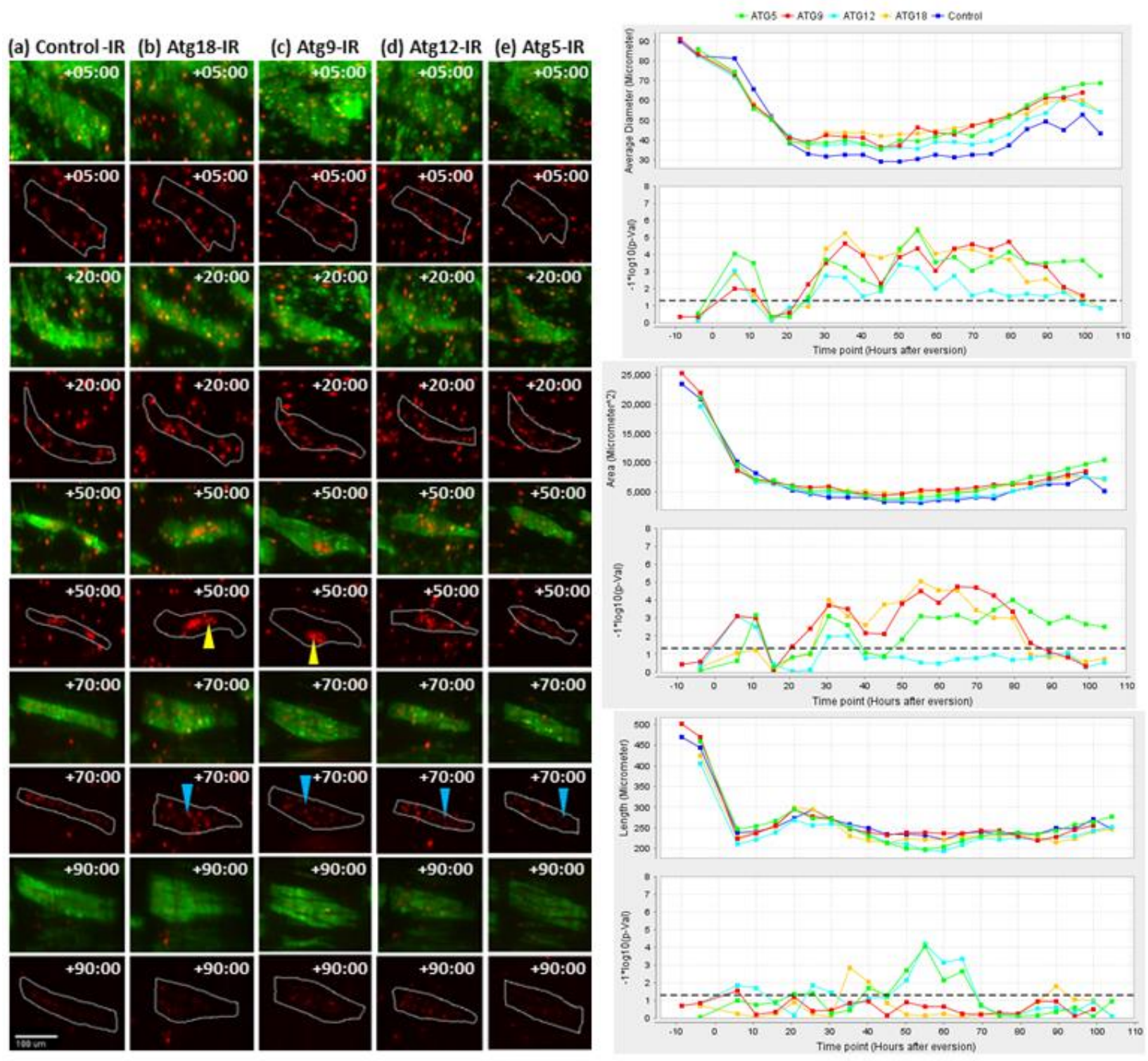

Figure 45. Atg5, Atg9, Atg12 and Atg18 promote atrophy and central clustering of nuclei in persistent muscles.

Left side of the figure shows the development of DIOM and nuclei distribution in control (a), Atg18 (b), $\operatorname{Atg} 9$ (c), Atg12 (d) and Atg5 (e). After +20 h, the muscle cells are hypertrophied for all Atgs. The yellow arrowhead shows the centrally clustered nuclei in $\operatorname{Atg} 9$ and $\operatorname{Atg} 18$ at $50 \mathrm{~h}$. During late stages, the nuclei in control form one row. Whereas in all Atg genes, the nuclei form two rows irrespective of muscle size (Shown by a cyan arrowhead). Right side of the figure compares the median values of three morphological features of muscle cells expressing different RNAi constructs: width (Top), area (Middle) and length (Bottom). For each population, statistics were derived from 10-20 muscle cells. The horizontal dotted lines in the significance graphs (bottom panels) represent the p-value 0.05. 

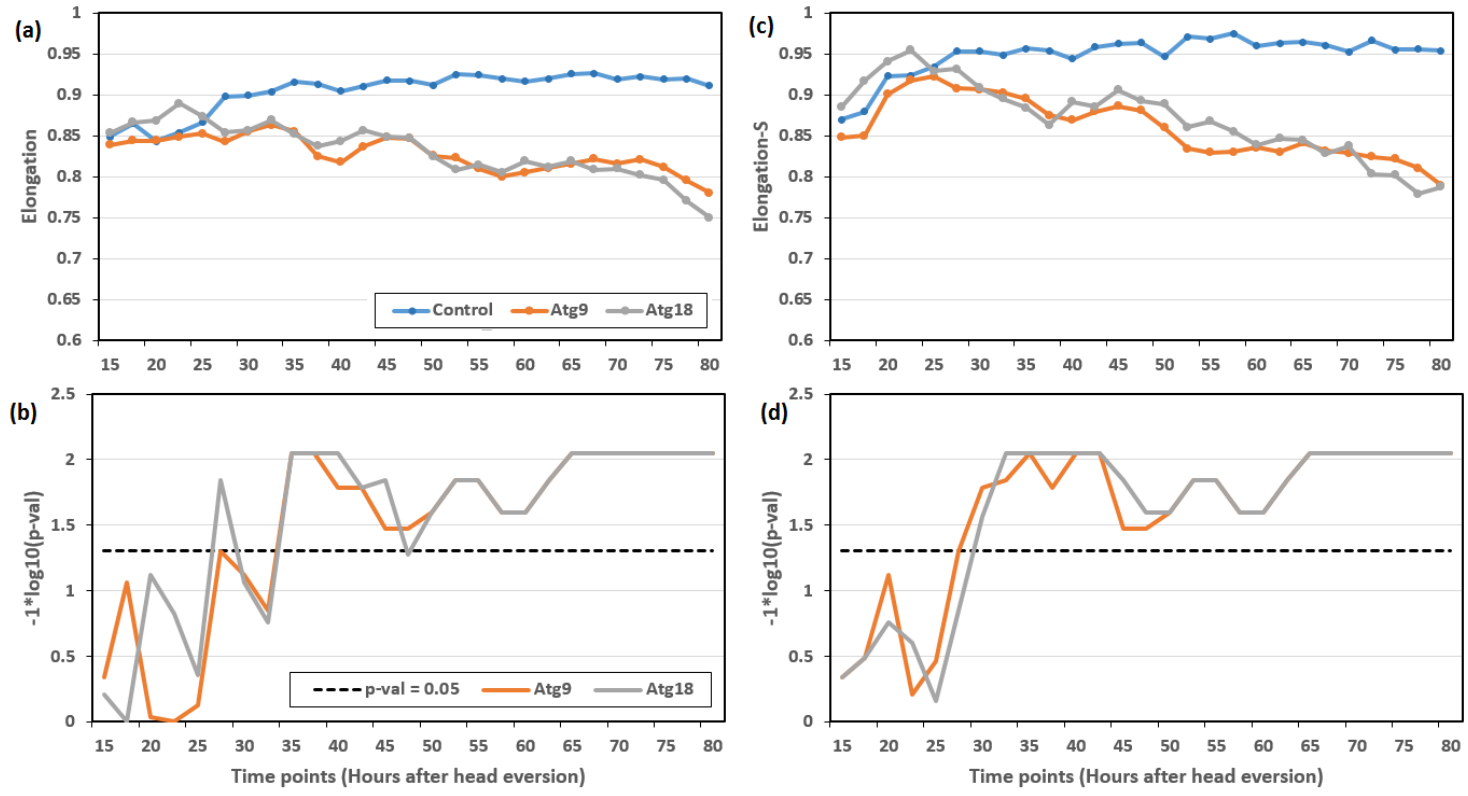

Figure 46.Comparison of median elongation (a) and elongation-S (c) in Atg9 and Atg18 RNAi.

For each population, statistics were derived from 5 muscle cells. (b \& d) Significance graph. The horizontal dotted lines in the significance graphs (bottom panel) represent the p-value 0.05.
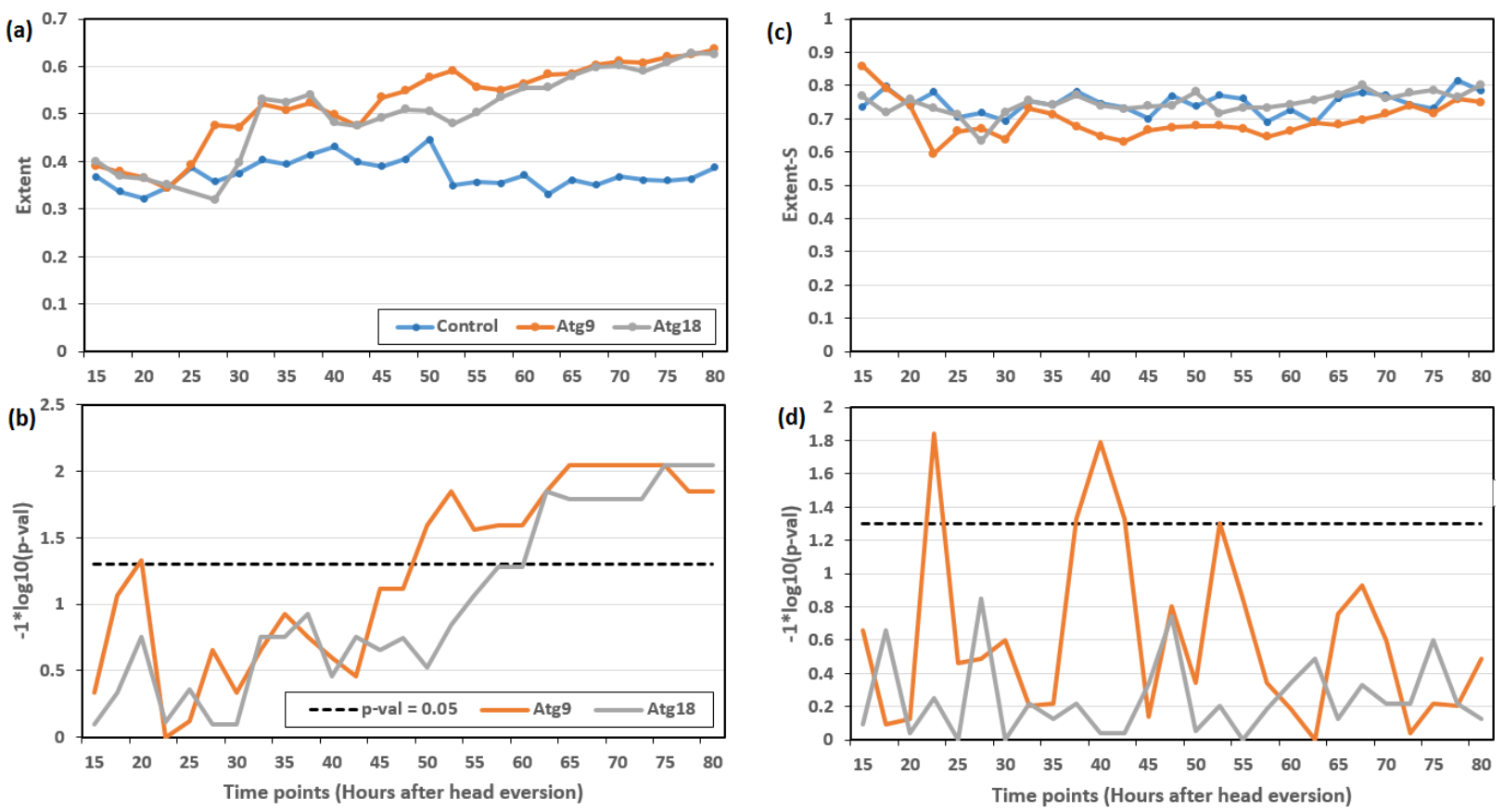

Figure 47. Average extent of muscle cells in Atg9 and Atg18 mutants.

a) Extent c) Extent-S. b, d) Significance graph. For each population, statistics were derived from 5-10 muscle cells. The horizontal dotted lines in the significance graphs represent the p-value 0.05. 


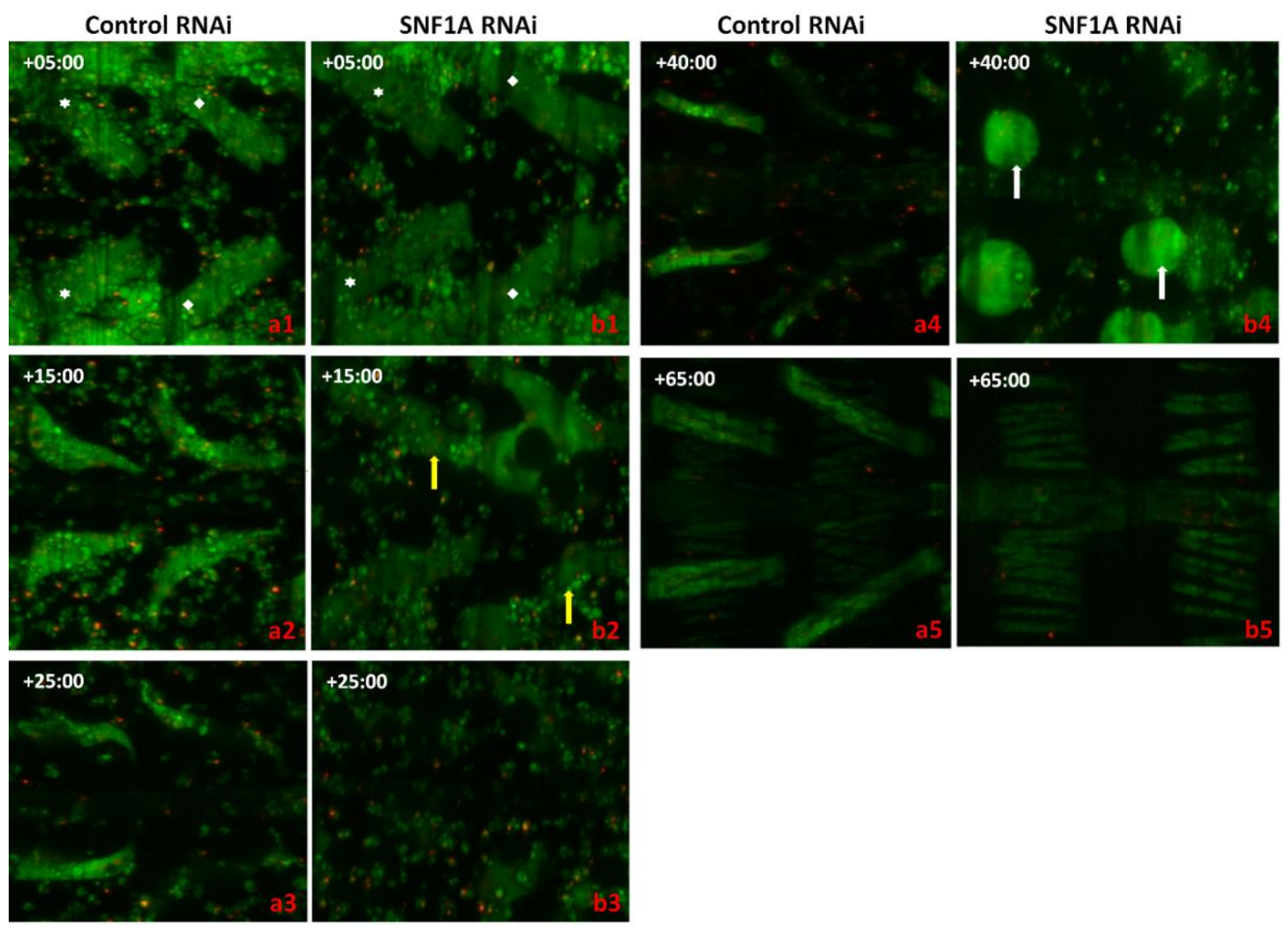

Figure 48. Silencing of SNF1A causes cell death of persistent muscles.

The figure shows the development of muscles (star-3rd segment, diamond-4th segment) in control (a) and SNF1A (b). The yellow arrow shows DIOMs undergoing cell death. The DIOMs form a circular structure before they are completely destroyed which is shown by the white arrow. 

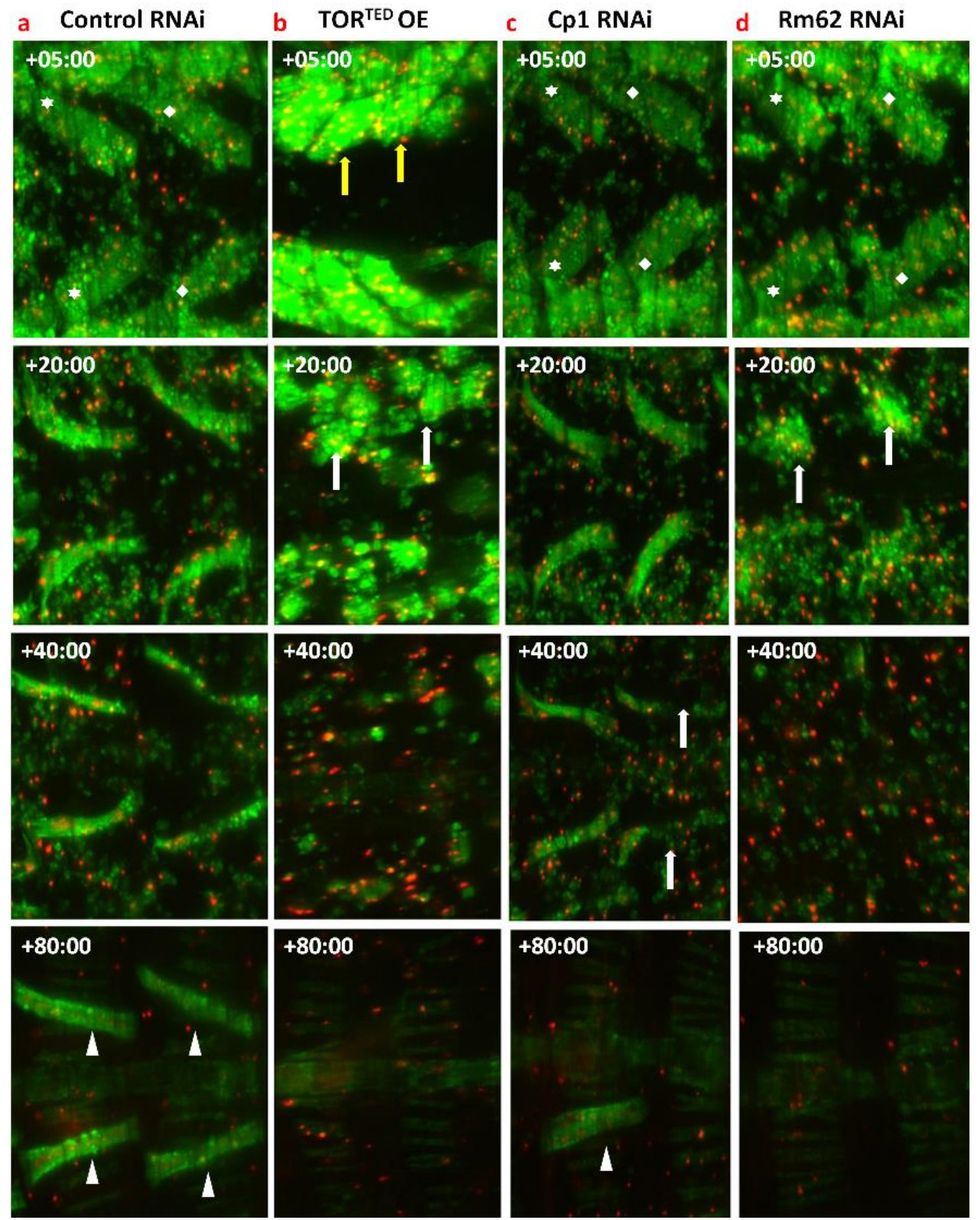

Figure 49. Silencing of $C P 1$ and $R M 62$ and over-expression of $T O R^{T E D}$ causes cell death of persistent muscles.

The figure shows the development of muscles (star- $3^{\text {rd }}$ segment, diamond- $4^{\text {th }}$ segment) in control (a), $T O R^{T E D}$ (b), CP1 (c) and RM62(d). The yellow arrow show delayed histolysis of DEOM due to overexpression of TOR ${ }^{T E D}$. The white arrow shows the cell death of DIOM. The time of death of DIOM is different in the three mutants. The surviving persistent muscle cells are shown by white arrowhead. 

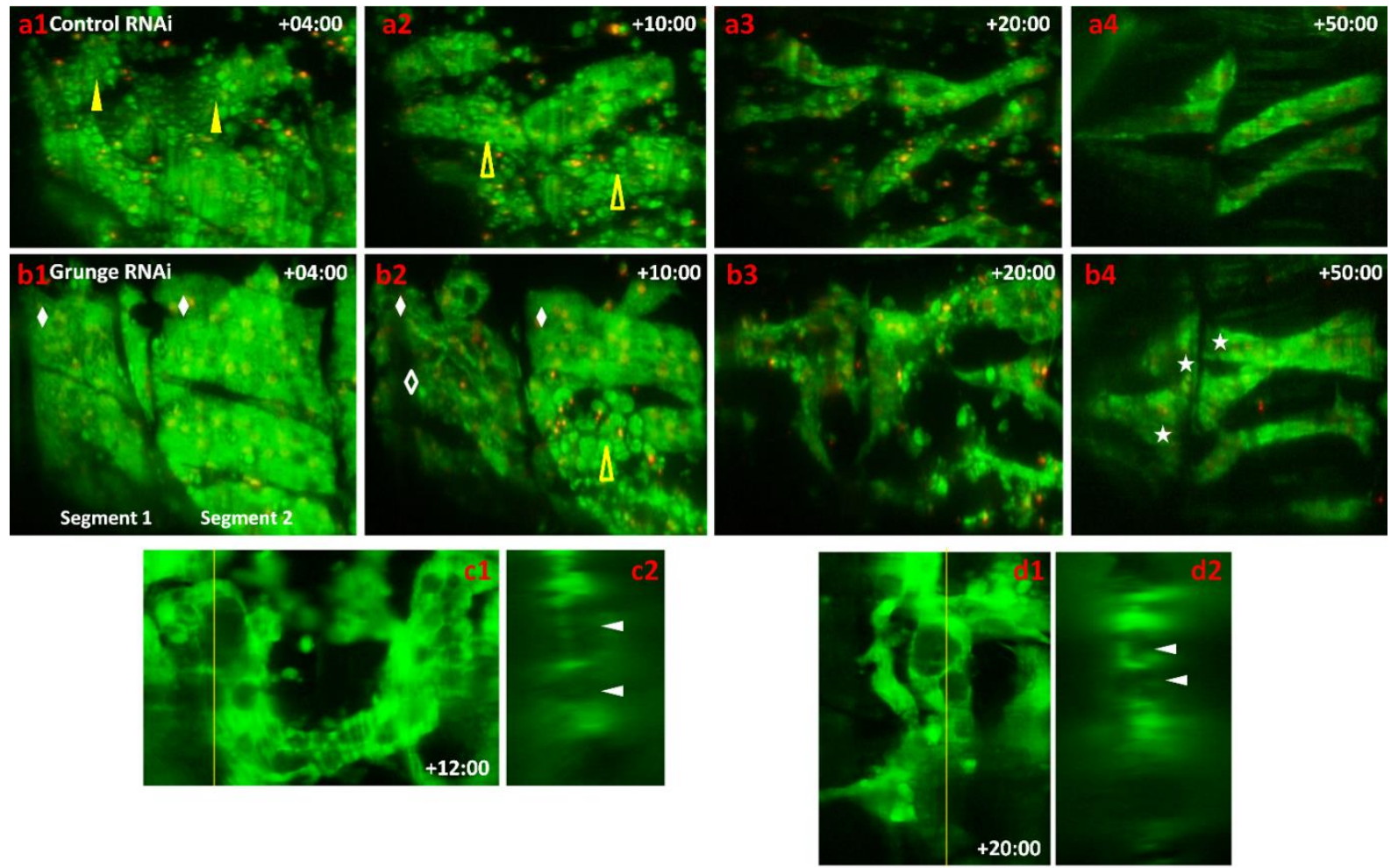

Figure 50.Grunge knockdown inhibits the histolysis of DEOMs in segment 1 and 2.

Top of the figure shows the development of muscles in control (a) and Grunge (b). The DEOM1 in segment 1 and 2 which escaped histolysis in Grunge mutant are indicated by a white diamond. DEOM2 in segment 2 undergoes histolysis (Yellow hollow arrowhead) whereas DEOM2 in segment 1 does not (White hollow diamond). The white stars in b4 show the adult DEOMs. c1 \& d1) Large vacuoles in the persisting DEOMs in Grunge mutant. The images are maximum intensity projection of 3D confocal stacks taken at 20x resolution. c2 \& d2) Orthogonal view of the DEOMs. White arrowhead indicates the vacuoles. 


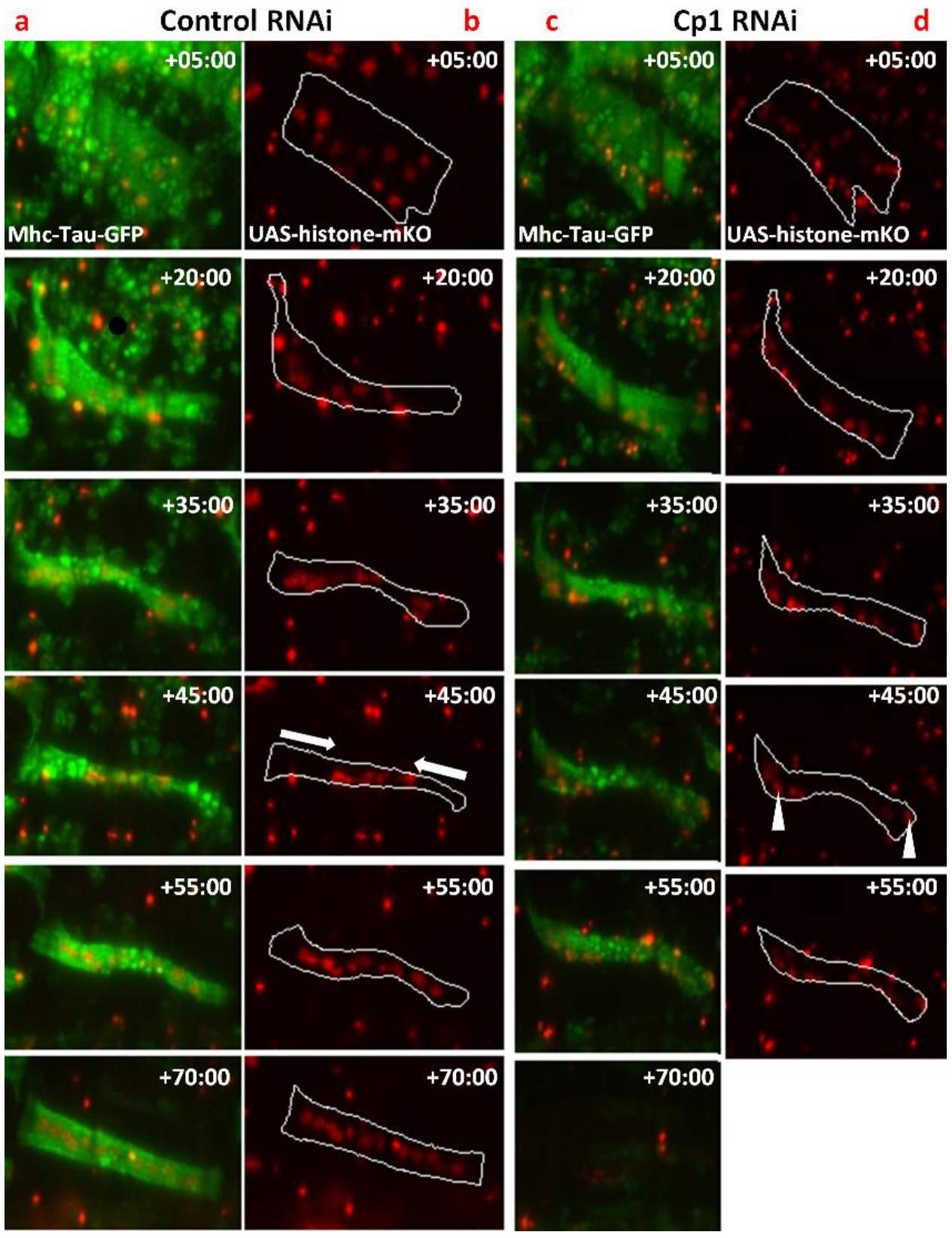

Figure 51. Silencing of $C p l$ affect the distribution of nuclei along the medial axis in persistent muscles.

(a) \& (b) In control, the nuclei move in anti-polar direction at $+45 \mathrm{~h}$ (White arrow) followed by polar migration. (c) \& (d) In Cpl RNAi, the anti-polar migration of nuclei is absent. At $+45 \mathrm{~h}$, the nuclei located at the poles is shown by white arrowheads. 

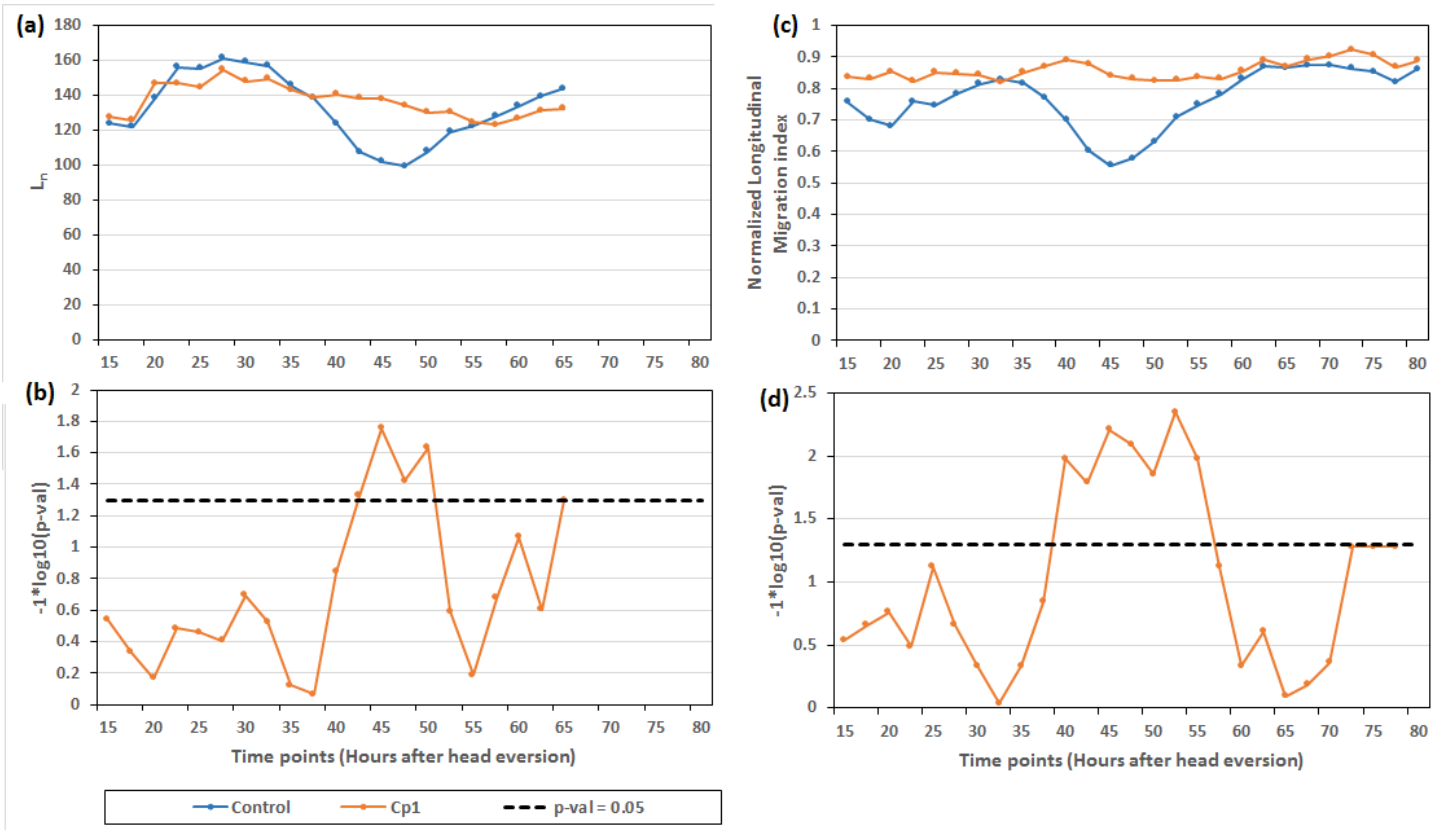

Figure 52. Longitudinal nuclear migration index help quantify the effect of $C p l$ knockdown on anti-polar migration of nuclei in persistent muscles.

(a) \& (c) Graphical comparisons of $L_{n}$ (Distance between the extremes of the nuclei along the medial axis) and normalized longitudinal migration index $N M_{\text {lon }}$ between control and $C p 1$ mutant. Due to absence of anti-polar movement of muscles around $+50 \mathrm{~h}$ in $C p l$ mutants; the value of both $L_{n}$ and $N M_{l o n}$ is high as compared to control. (b) \& (d) Significance graph. For each population, statistics were derived from the same DIOM1 used in previous analysis. The graph on top show average values of the features. The horizontal dotted lines in the significance graphs represent the p-value 0.05. 

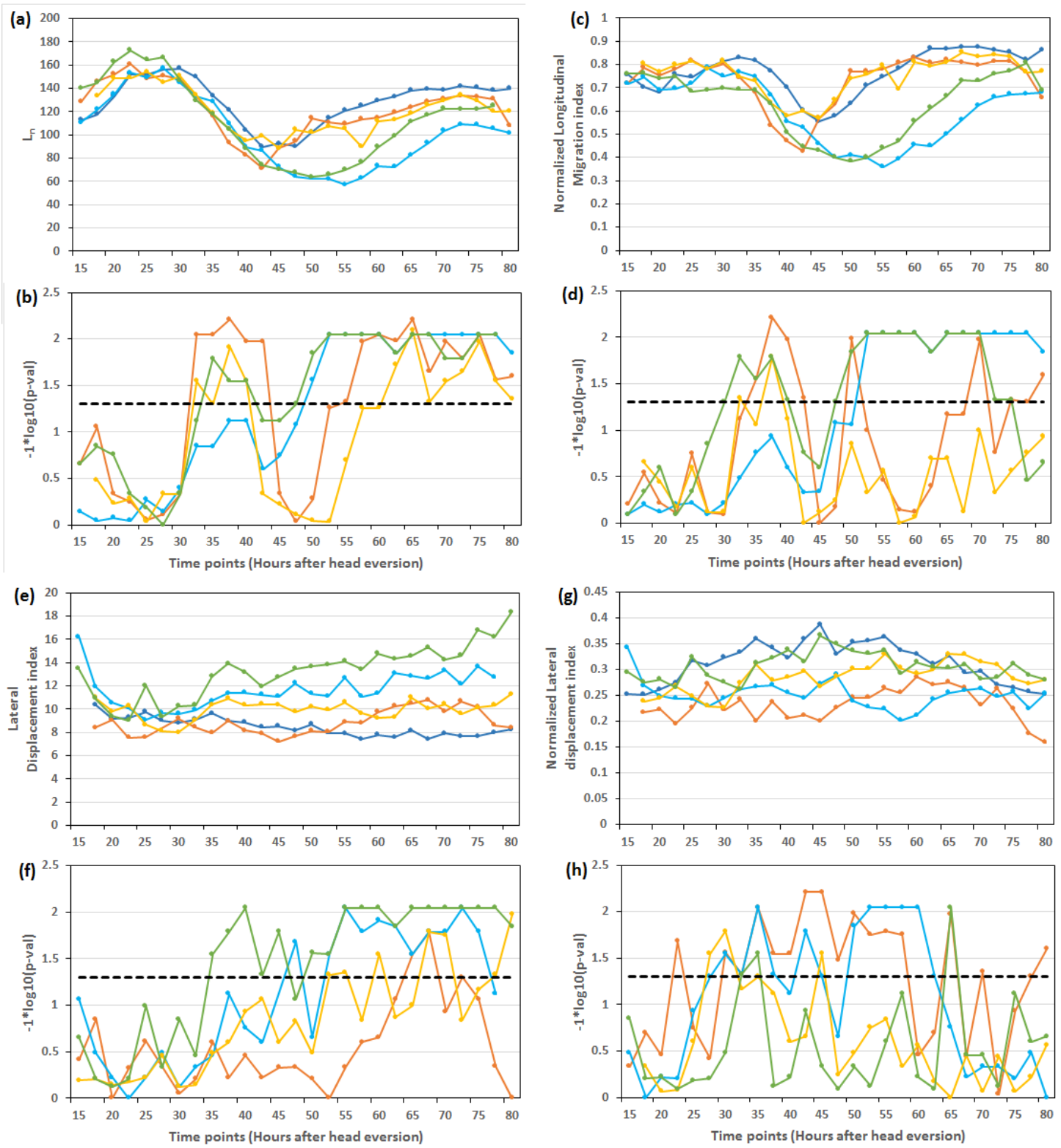

$\rightarrow$ Control $\longrightarrow$ Atg5 $\longrightarrow$ Atg9 $\multimap$ Atg12 $\multimap$ Atg18 $\because$ - p-val $=0.05$

Figure 53. Nuclear spatial pattern features help quantify the effect of autophagy related genes knockdown on nuclear distribution in persistent muscles.

The figure compares following nuclear pattern features in muscles expressing Atg5 RNAi, Atg9 RNAi, Atg12 RNAi, Atg18 RNAi and control: a) $L_{n}$. c) Normalized longitudinal migration index. e) Lateral displacement index. g) Normalized lateral displacement index. b, d, f, h) Significance graph. For each population, statistics were derived from the same DIOM1 used in previous analysis. The graph on top show average values of the features. The horizontal dotted lines in the significance graphs (bottom panels) represent the p-value 0.05 . 

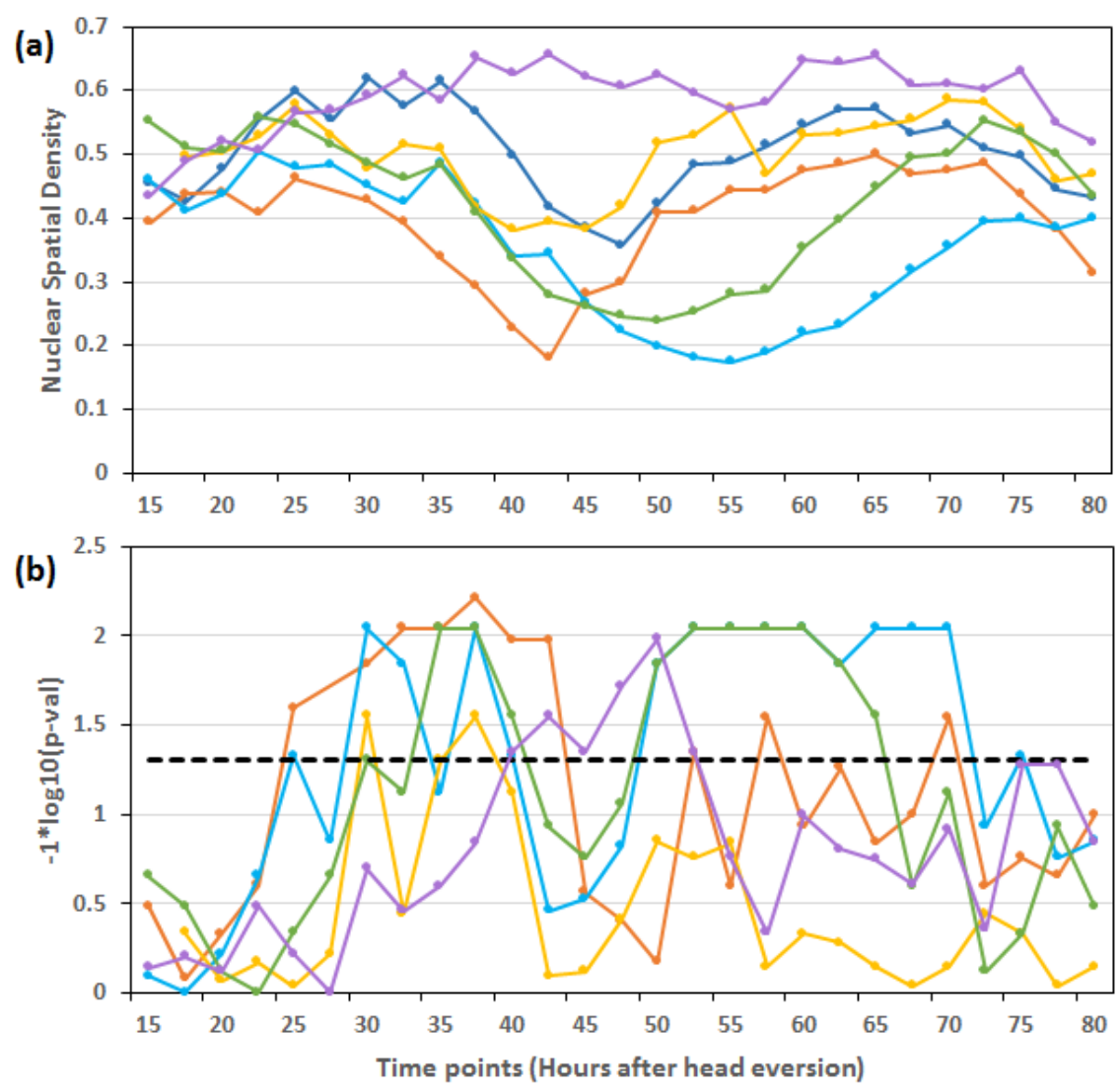

-Control —Atg5 —Atg9 —Atg12 —Atg18 —Cp1 - p-val $=0.05$

Figure 54. Spatial nuclear density index help quantify the distribution of nuclei with respect to the muscle mass change.

(a) The figure compares average nuclear density index of nuclei in muscles expressing Cp1 RNAi, Atg5 RNAi, Atg9 RNAi, Atg 12 RNAi, Atg 18 RNAi and control. (b) Significance graph. Statistics were derived from 5, 7 and 6 DIOMs for control, Cpl and Atgs respectively. The muscles were from segment 3 of abdomen. The horizontal dotted lines in the significance graphs (bottom panels) represent the p-value 0.05 . 
(a)

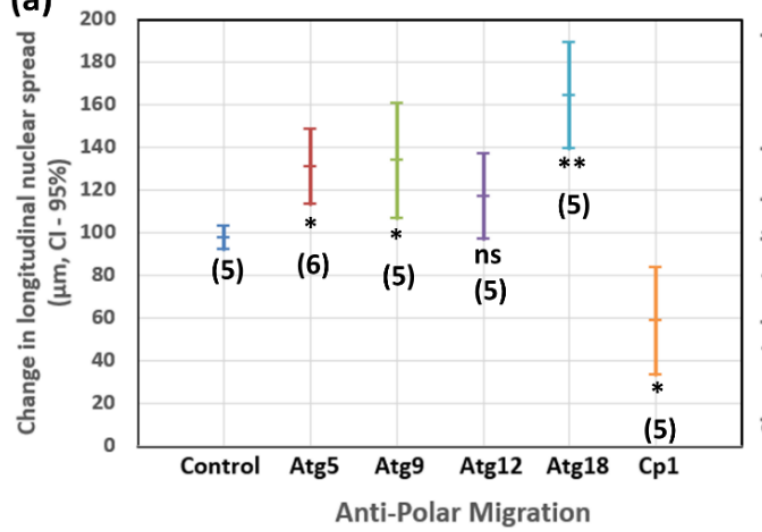

(b)

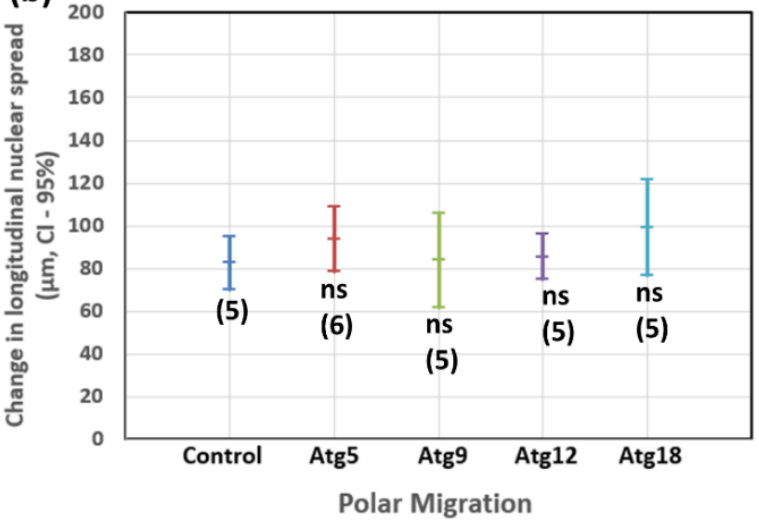

Figure 55. Comparison of change in longitudinal nuclear spread during polar and anti-polar migration between different genotypes 


\section{Chapter 7 Discussions and Conclusions}

\subsection{Major Contributions}

From biological perspective, our major contribution has been the design of a framework for skeletal muscle research using muscle remodeling during Drosophila metamorphosis as a model. The primary objective of this framework i.e. phenotypic analysis of genetic perturbations on the development of persistent muscles, was achieved by combining RNAi based gene silencing with time lapse in vivo imaging. The ability of fluorescent markers to visualize a specific biological process without affecting the global physiology of cell has made it an ideal tool for studying gene functions in live muscle cells. In our study, we used two markers in one reporter line to visualize both muscles and myonuclei and by using UAS-GAL4 system we assured muscle specific expression of dsRNA and $\mathrm{mKO}$ fluorophore (for visualizing myonuclei) In order to characterize the muscle phenotypes caused by genetic perturbation in large number of genes, we designed an efficient two-level phenotypic screening system. At level-1 screening, preliminary phenotypic analysis was done using macro zoom microscope to shortlist genetic perturbations which affect muscle morphology or myonuclear distribution. Performing confocal microscopy, which is not a high throughput technique, for every genetic perturbation is not a feasible idea. Therefore, by taking images at fewer time intervals using macro zoom microscope for preliminary phenotypic analysis, we saved both resources and time. The shortlisted genes were further analyzed quantitatively using time lapse confocal imaging in level2 screening.

The quantitative analysis of time lapse confocal images is the secondary objective of the framework for skeletal muscle research. The quantitative phenotypic analysis of large number of images generated during 4-5 days time lapse confocal imaging of Drosophila metamorphosis requires high throughput image analysis algorithms and structured data storage. Therefore, I developed the Fly Muscle Analysis in Java (FMAj) software tool for the quantitative characterization of muscle phenotypes in time series images. To effectively perform comparative phenotypic profiling of muscle 
development, I integrated image processing, segmentation, structured storage by MySQL and statistical analysis. The integration of multiple tasks in a single platform enhances productivity as the alternative of exporting ROI data and manual processing in a spreadsheet program would be more time-consuming. Since FMAj uses ImageJ library, it is easier to enhance its functionalities by incorporating additional image processing and computer vision algorithms. The two newly designed muscle analysis algorithms were implemented in FMAj i.e. muscle segmentation for morphological analysis and myonuclei spatial pattern analysis.

For morphological analysis of muscles, a segmentation method was required which can extract the muscle boundaries in large number of time series images with minimal manual intervention. A new muscle segmentation was developed which first divided the image into edge-preserving regions using watershed algorithm, and then, classified these regions into muscle and non-muscle classes on the basis of shape and edge confidence map. The advantage of my method is three-fold: Firstly better results were achieved because the classification of the regions is constrained by the shape of muscle cell from the previous time point and thus resulting in fewer false classification of debris as muscle. Secondly, the confidence map component of the dissimilarity metric increased the robustness of method by ensuring that the edges with high probability of being a part of muscle boundary were not falsely removed because of slightly high shape dissimilarity. Thus, by balancing the temporal shape based distance and edge confidence value in the region classification algorithm, high segmentation accuracy was obtained. The last advantage of watershed based segmentation method is the faster processing time due to minimal user intervention.

To better understand the process of nuclear migration in muscle cells, I also designed algorithms to extract set of nuclear spatial pattern features which includes nuclear segmentation, tracking and classification. All steps including nuclear segmentation and tracking are performed in a fully automated fashion, thus enabling a more reproducible analysis of sizeable time-series image data. Nuclear classification results were improved by introducing a tracking based algorithm which exploits the differences in motion of external and internal nuclei to classify them. It is also robust enough to reduce classification errors during large movements of the muscle cell. Multiple nuclear spatial pattern features were designed, each catering to a specific type 
of nuclear distribution. The nuclear spatial density index measures how densely the nuclei are packed; however, they cannot quantify the localization of the nuclei in the muscle cell. To accomplish this, new nuclear features were designed that can quantify the distribution of nuclei along the medial axis and along the width of the muscle cell, called as longitudinal migration index and lateral displacement index respectively.

Using FMAj tool for characterization of the muscle phenotypes, we found new phenotypes for genes which have not been studied before in muscles. We found that the silencing of Atg genes (Atg5, Atg9, Atg12 and Atg18) caused supression of atrophy in DIOMs in transient manner. During metamorphosis in Drosophila, autophagy does not regulate atrophy throughout metamorphosis and the degree of hypertrophy varies between the four Atg genes. However, it is interesting that the genes mentioned above do not affect the histolysis of DEOMs and the development of IFMs. The reason for selective regulation of atrophy in muscles is unclear. We also found that SNF1A and Grunge participate in muscle remodeling during metamorphosis. SNF1A/AMPK is a negative regulator of cell growth. It inhibits Tor by phosphorylation of Tsc2 and Raptor to regulate autophagy [144], [145]. In a recent study, direct association between SNF1A with Atgl has been found [146]. Contrary to the previous results, SNF1A silencing resulted in cell death of persistent muscles instead of decrease in autophagy. Unlike SNF1A, silencing of Grunge was associated with selective inhibition of DEOMs histolysis. Grunge (Gug), the only member of atrophin family in Drosophila, is an important regulator of embryonic segmentation [147]. It has also been found that Grunge plays an important role in autophagic cell death of salivary gland during metamorphosis [148]; however its role in muscle remodeling during metamorphosis is unclear. Consistent with previous study on salivary gland, we found that Grunge plays a role in cell death of DEOMs. The results of our study also indicate that the activity of Grunge in DEOMs might be dependent on the abdominal segment in which the muscle is located. We also observed that the knockdown of Grunge resulted in formation of large vacuoles in DEOMs. The formation of vacuoles has been observed during the programmed cell death of salivary gland in Drosophila [149]. Vacuole formation in muscles has been associated with accumulation of lysosomal vesicles containing glycogen and it has been observed in autophagic vacuolar myopathies like pompe and danon disease [150]. 
The abnormal nuclei arrangement in $C p 1$ and Atgs RNAi were analyzed statistically using nuclear spatial pattern features. According to myonuclear domain theory nuclei should be evenly distributed in healthy muscle [31]; a phenotype we observed in $C p 1$ mutant. Whereas central positioning of nuclei, which has been associated with Central nuclear myopathies, was observed in control and Atgs RNAi during the mid-pupal stage. Knockdown of $\operatorname{Atg} 9$ and $\operatorname{Atg} 18$ RNAi resulted in more densely packed (longitudinally) nuclei as compared to control. This indicates that $\operatorname{Atg} 9$ and $\operatorname{Atg} 18$ help in the central positioning of nuclei by regulating their anti-polar migration. We found that the effect of silencing of genes on nuclear migration is more prominent in $\operatorname{Atg} 9$ and Atg18 as compared to Atg5 and Atg12. Atgs were also involved in positioning of nuclei along the width of muscle.

The in vivo visualization system and the custom image processing tool (FMAj) provides a unique platform for uncovering new insights into the regulation of muscle wasting in Drosophila during metamorphosis. The phenotypic profiling of the initial set of genes prove that metamorphosis in Drosophila can be used to study the genes regulating muscle mass. In summary, our model can fill knowledge gaps and propose new hypotheses in the arena of muscle wasting research.

\subsection{Future Work}

In image processing pipeline, further work is required to improve the segmentation methodology to handle cases where some sections of muscle are misclassified as debris due to very low intensity. In future, the parameters used in segmentation could be learned to improve the accuracy of region classification. Features to measure the amount of debris in the pupa could also be designed. Further comparative analysis of nuclear spatial pattern in Atg genes is required. Various improvements can be made in FMAj like implementing a segmentation editor to remove outliers, handling samples from same genotype imaged at different temperatures.

More research is required to find the molecular pathways through which genes like SNF1A and Grunge control cell death in muscles. The opposite nuclear phenotypes observed in $\operatorname{Atg} 9, \operatorname{Atg} 18$ and $C p 1$ mutants raises lot of questions about the association 
between nuclei distribution and muscle wasting. Further work is required to understand the molecular mechanisms behind the migratory patterns of nuclei in muscles. 


\section{References}

[1] T. Shavlakadze and M. Grounds, "Of bears, frogs, meat, mice and men: complexity of factors affecting skeletal muscle mass and fat," BioEssays, vol. 28, no. 10, pp. 994-1009, Oct. 2006.

[2] D. J. Glass, "Signalling pathways that mediate skeletal muscle hypertrophy and atrophy," Nat. Cell Biol., vol. 5, no. 2, pp. 87-90, Feb. 2003.

[3] S. Schiaffino and C. Mammucari, "Regulation of skeletal muscle growth by the IGF1-Akt/PKB pathway: insights from genetic models," Skelet. Muscle, vol. 1, no. 1, p. 4, 2011.

[4] R. Kambadur, M. Sharma, T. P. Smith, and J. J. Bass, "Mutations in myostatin (GDF8) in double-muscled Belgian Blue and Piedmontese cattle," Genome Res., vol. 7, no. 9, pp. 910-916, Sep. 1997.

[5] W. A. Dunn, "Autophagy and related mechanisms of lysosome-mediated protein degradation," Trends Cell Biol., vol. 4, no. 4, pp. 139-143, Apr. 1994.

[6] D. J. Klionsky et al., "A unified nomenclature for yeast autophagy-related genes," Dev. Cell, vol. 5, no. 4, pp. 539-545, Oct. 2003.

[7] M. Sandri, “Autophagy in skeletal muscle," FEBS Lett., vol. 584, no. 7, pp. 14111416, Apr. 2010.

[8] P. Codogno and A. J. Meijer, "Autophagy and signaling: their role in cell survival and cell death," Cell Death Differ., vol. 12 Suppl 2, pp. 1509-1518, Nov. 2005.

[9] M. Sandri, "Autophagy in health and disease. 3. Involvement of autophagy in muscle atrophy," Am. J. Physiol. Cell Physiol., vol. 298, no. 6, pp. C1291-1297, Jun. 2010.

[10] S. E. Wohlgemuth, A. Y. Seo, E. Marzetti, H. A. Lees, and C. Leeuwenburgh, "Skeletal muscle autophagy and apoptosis during aging: effects of calorie restriction and life-long exercise," Exp. Gerontol., vol. 45, no. 2, pp. 138-148, Feb. 2010.

[11] K. Weigmann et al., "FlyMove--a new way to look at development of Drosophila," Trends Genet. TIG, vol. 19, no. 6, pp. 310-311, Jun. 2003.

[12] E. H. Chen and E. N. Olson, "Towards a molecular pathway for myoblast fusion in Drosophila," Trends Cell Biol., vol. 14, no. 8, pp. 452-460, Aug. 2004.

[13] M. K. Baylies, M. Bate, and M. Ruiz Gomez, "Myogenesis: a view from Drosophila," Cell, vol. 93, no. 6, pp. 921-927, Jun. 1998.

[14] H. Sink, Ed., Muscle development in drosophila. Georgetown, Tex. : New York, N.Y: Landes Bioscience/Eurekah.com ; Springer Science+Business Media, 2006.

[15] C. W. Robertson, "The metamorphosis of Drosophila melanogaster, including an accurately timed account of the principal morphological changes," J. Morphol., vol. 59, no. 2, pp. 351-399, Jun. 1936.

[16] M. Bate and A. Martinez Arias, Eds., "The mesoderm and its derivatives," in The development of Drosophila melanogaster, Plainview, NY: Cold Spring Harbor Laboratory Press, 1993, pp. 1013-1090.

[17] D. Bodenstein, "The Postembryonic Development of Drosophila," in Biology of Drosophila, M. Demerec, Ed. New York and London: Hafner Publishing Company, 1965, pp. 275-367. 
[18] C. Jiang, E. H. Baehrecke, and C. S. Thummel, "Steroid regulated programmed cell death during Drosophila metamorphosis," Dev. Camb. Engl., vol. 124, no. 22, pp. 4673-4683, Nov. 1997.

[19] E. H. Baehrecke, "Autophagic programmed cell death in Drosophila," Cell Death Differ., vol. 10, no. 9, pp. 940-945, Sep. 2003.

[20] C. Jiang, A. F. Lamblin, H. Steller, and C. S. Thummel, "A steroid-triggered transcriptional hierarchy controls salivary gland cell death during Drosophila metamorphosis," Mol. Cell, vol. 5, no. 3, pp. 445-455, Mar. 2000.

[21] J. Zirin et al., "Ecdysone signaling at metamorphosis triggers apoptosis of Drosophila abdominal muscles," Dev. Biol., vol. 383, no. 2, pp. 275-284, Nov. 2013.

[22] T. E. Rusten et al., "Programmed autophagy in the Drosophila fat body is induced by ecdysone through regulation of the PI3K pathway," Dev. Cell, vol. 7, no. 2, pp. 179-192, Aug. 2004.

[23] T. N. Stitt et al., "The IGF-1/PI3K/Akt Pathway Prevents Expression of Muscle Atrophy-Induced Ubiquitin Ligases by Inhibiting FOXO Transcription Factors," Mol. Cell, vol. 14, no. 3, pp. 395-403, May 2004.

[24] C. K. McPhee and E. H. Baehrecke, "Autophagy in Drosophila melanogaster," Biochim. Biophys. Acta BBA - Mol. Cell Res., vol. 1793, no. 9, pp. 1452-1460, Sep. 2009.

[25] "OrthoDisease: Human Disease Orthologs Database." [Online]. Available: http://orthodisease.sbc.su.se/cgi-bin/index.cgi. [Accessed: 24-Sep-2015].

[26] W. Brogiolo, H. Stocker, T. Ikeya, F. Rintelen, R. Fernandez, and E. Hafen, “An evolutionarily conserved function of the Drosophila insulin receptor and insulinlike peptides in growth control," Curr. Biol. CB, vol. 11, no. 4, pp. 213-221, Feb. 2001.

[27] J. Zirin and N. Perrimon, "Drosophila as a model system to study autophagy," Semin. Immunopathol., vol. 32, no. 4, pp. 363-372, Dec. 2010.

[28] R. S. Garofalo, "Genetic analysis of insulin signaling in Drosophila," Trends Endocrinol. Metab. TEM, vol. 13, no. 4, pp. 156-162, Jun. 2002.

[29] E. Bier, "Drosophila, the golden bug, emerges as a tool for human genetics," Nat. Rev. Genet., vol. 6, no. 1, pp. 9-23, Jan. 2005.

[30] T. R. Gregory, "Coincidence, coevolution, or causation? DNA content, cell size, and the C-value enigma," Biol. Rev. Camb. Philos. Soc., vol. 76, no. 1, pp. 65101, Feb. 2001.

[31] Z. W. Hall and E. Ralston, "Nuclear domains in muscle cells," Cell, vol. 59, no. 5, pp. 771-772, Dec. 1989.

[32] C. Priester, L. C. Morton, S. T. Kinsey, W. O. Watanabe, and R. M. Dillaman, "Growth patterns and nuclear distribution in white muscle fibers from black sea bass, Centropristis striata: evidence for the influence of diffusion," J. Exp. Biol., vol. 214, no. 8, pp. 1230-1239, Apr. 2011.

[33] J. C. Bruusgaard, K. Liestøl, M. Ekmark, K. Kollstad, and K. Gundersen, "Number and spatial distribution of nuclei in the muscle fibres of normal mice studied in vivo," J. Physiol., vol. 551, no. 2, pp. 467-478, Sep. 2003.

[34] D. L. Allen, R. R. Roy, and V. R. Edgerton, "Myonuclear domains in muscle adaptation and disease," Muscle Nerve, vol. 22, no. 10, pp. 1350-1360, Oct. 1999.

[35] K. I. Wada, S. Katsuta, and H. Soya, "Natural occurrence of myofiber cytoplasmic enlargement accompanied by decrease in myonuclear number," Jpn. J. Physiol., vol. 53, no. 2, pp. 145-150, Apr. 2003. 
[36] H. Jungbluth and M. Gautel, "Pathogenic mechanisms in centronuclear myopathies," Front. Aging Neurosci., vol. 6, p. 339, 2014.

[37] L. Gueneau et al., "Mutations of the FHL1 Gene Cause Emery-Dreifuss Muscular Dystrophy," Am. J. Hum. Genet., vol. 85, no. 3, pp. 338-353, Sep. 2009.

[38] H.-H. Li et al., "[Adeno-associated virus vector carrying human minidystrophin gene SMCKA3999 effectively ameliorates dystrophic pathology in mdx model mice]," Zhonghua Yi Xue Za Zhi, vol. 83, no. 17, pp. 1513-1516, Sep. 2003.

[39] X. Zhang et al., "Syne-1 and Syne-2 play crucial roles in myonuclear anchorage and motor neuron innervation," Dev. Camb. Engl., vol. 134, no. 5, pp. 901-908, Mar. 2007.

[40] D. A. Starr and H. N. Fridolfsson, "Interactions between nuclei and the cytoskeleton are mediated by SUN-KASH nuclear-envelope bridges," Annu. Rev. Cell Dev. Biol., vol. 26, pp. 421-444, 2010.

[41] H. Elhanany-Tamir, Y. V. Yu, M. Shnayder, A. Jain, M. Welte, and T. Volk, "Organelle positioning in muscles requires cooperation between two KASH proteins and microtubules," J. Cell Biol., vol. 198, no. 5, pp. 833-846, Sep. 2012.

[42] V. K. Schulman, E. S. Folker, J. N. Rosen, and M. K. Baylies, "Syd/JIP3 and JNK Signaling Are Required for Myonuclear Positioning and Muscle Function," PLoS Genet., vol. 10, no. 12, p. e1004880, Dec. 2014.

[43] "Milestones in light microscopy," Nat. Cell Biol., vol. 11, no. 10, p. 1165, Oct. 2009.

[44] "Nikon MicroscopyU | Stereomicroscopy | Darkfield Illumination." [Online]. Available:

http://www.microscopyu.com/articles/stereomicroscopy/stereodarkfield.html. [Accessed: 03-Oct-2015].

[45] "Nikon MicroscopyU | Phase Contrast Microscopy." [Online]. Available: http://www.microscopyu.com/articles/phasecontrast/index.html. [Accessed: 03Oct-2015].

[46] "Nikon MicroscopyU | Concepts and Formulas in Microscopy." [Online]. Available: http://www.microscopyu.com/articles/formulas/. [Accessed: 03-Oct2015].

[47] “Olympus Microscopy Resource Center | Fluorescence Microscopy." [Online]. Available:

http://www.olympusmicro.com/primer/techniques/fluorescence/fluorhome.html. [Accessed: 03-Oct-2015].

[48] “Olympus Microscopy Resource Center | Confocal Microscopy - Fluorophores for Confocal Microscopy." [Online]. Available: http://www.olympusmicro.com/primer/techniques/confocal/fluorophoresintro.ht ml. [Accessed: 24-Sep-2015].

[49] "Molecular Expressions Microscopy Primer: Specialized Microscopy Techniques - Confocal Microscopy." [Online]. Available: http://micro.magnet.fsu.edu/primer/techniques/confocal/index.html. [Accessed: 24-Sep-2015].

[50] J. Huisken, "Optical Sectioning Deep Inside Live Embryos by Selective Plane Illumination Microscopy," Science, vol. 305, no. 5686, pp. 1007-1009, Aug. 2004.

[51] W. Denk, J. Strickler, and W. Webb, "Two-photon laser scanning fluorescence microscopy," Science, vol. 248, no. 4951, pp. 73-76, Apr. 1990. 
[52] B. Neumann et al., "Phenotypic profiling of the human genome by time-lapse microscopy reveals cell division genes," Nature, vol. 464, no. 7289, pp. 721-727, Apr. 2010.

[53] J. Bai, R. Binari, J.-Q. Ni, M. Vijayakanthan, H.-S. Li, and N. Perrimon, "RNA interference screening in Drosophila primary cells for genes involved in muscle assembly and maintenance," Dev. Camb. Engl., vol. 135, no. 8, pp. 1439-1449, Apr. 2008.

[54] R. E. Ward, P. Reid, A. Bashirullah, P. P. D’Avino, and C. S. Thummel, "GFP in living animals reveals dynamic developmental responses to ecdysone during

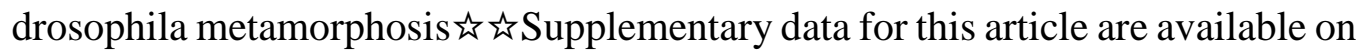
Science Direct (http://www.sciencedirect.com ) and on the author's web site (http://thummel.genetics.utah.edu/).," Dev. Biol., vol. 256, no. 2, pp. 389-402, Apr. 2003.

[55] C. Vinegoni, C. Pitsouli, D. Razansky, N. Perrimon, and V. Ntziachristos, "In vivo imaging of Drosophila melanogaster pupae with mesoscopic fluorescence tomography," Nat. Methods, vol. 5, no. 1, pp. 45-47, Dec. 2007.

[56] B. Sönnichsen et al., "Full-genome RNAi profiling of early embryogenesis in Caenorhabditis elegans," Nature, vol. 434, no. 7032, pp. 462-469, Mar. 2005.

[57] L. Pelkmans et al., "Genome-wide analysis of human kinases in clathrin- and caveolae/raft-mediated endocytosis," Nature, vol. 436, no. 7047, pp. 78-86, Jul. 2005.

[58] B. Neumann et al., "High-throughput RNAi screening by time-lapse imaging of live human cells," Nat. Methods, vol. 3, no. 5, pp. 385-390, May 2006.

[59] M. Held et al., "CellCognition: time-resolved phenotype annotation in highthroughput live cell imaging," Nat. Methods, vol. 7, no. 9, pp. 747-754, Sep. 2010.

[60] Bo Peng, Lei Zhang, and D. Zhang, "Automatic Image Segmentation by Dynamic Region Merging," IEEE Trans. Image Process., vol. 20, no. 12, pp. 3592-3605, Dec. 2011.

[61] S. Beucher and C. Lantuejoul, "Use of watersheds in contour detection," Proc Int Workshop Image Process. Real-Time Edge Motion Detect., 1979.

[62] L. Vincent and P. Soille, "Watersheds in digital spaces: an efficient algorithm based on immersion simulations," IEEE Trans. Pattern Anal. Mach. Intell., vol. 13, no. 6, pp. 583-598, Jun. 1991.

[63] R. C. Gonzalez and R. E. Woods, Digital image processing, 3rd ed. Upper Saddle River, N.J: Prentice Hall, 2008.

[64] J. Canny, "A Computational Approach to Edge Detection," IEEE Trans. Pattern Anal. Mach. Intell., vol. PAMI-8, no. 6, pp. 679-698, Nov. 1986.

[65] M. Kass, A. Witkin, and D. Terzopoulos, "Snakes: Active contour models," Int. J. Comput. Vis., vol. 1, no. 4, pp. 321-331, Jan. 1988.

[66] J. A. Sethian and J. A. Sethian, Level set methods and fast marching methods: evolving interfaces in computational geometry, fluid mechanics, computer vision, and materials science, 2nd ed. Cambridge, U.K.; New York: Cambridge University Press, 1999.

[67] Z. Wu and R. Leahy, "An optimal graph theoretic approach to data clustering: theory and its application to image segmentation," IEEE Trans. Pattern Anal. Mach. Intell., vol. 15, no. 11, pp. 1101-1113, Nov. 1993.

[68] Jianbo Shi and J. Malik, "Normalized cuts and image segmentation," 1997, pp. 731-737. 
[69] Q. Zhong, A. G. Busetto, J. P. Fededa, J. M. Buhmann, and D. W. Gerlich, "Unsupervised modeling of cell morphology dynamics for time-lapse microscopy," Nat. Methods, vol. 9, no. 7, pp. 711-713, May 2012.

[70] R. Pepperkok and J. Ellenberg, "High-throughput fluorescence microscopy for systems biology," Nat. Rev. Mol. Cell Biol., vol. 7, no. 9, pp. 690-696, Sep. 2006.

[71] T. Walter et al., "Automatic identification and clustering of chromosome phenotypes in a genome wide RNAi screen by time-lapse imaging," J. Struct. Biol., vol. 170, no. 1, pp. 1-9, Apr. 2010.

[72] H. Lee et al., "Quantification and clustering of phenotypic screening data using time-series analysis for chemotherapy of schistosomiasis," BMC Genomics, vol. 13, no. Suppl 1, p. S4, 2012.

[73] D. Dutta, J. W. Bloor, M. Ruiz-Gomez, K. VijayRaghavan, and D. P. Kiehart, "Real-time imaging of morphogenetic movements in Drosophila using Gal4UAS-driven expression of GFP fused to the actin-binding domain of moesin," Genes. N. Y. N 2000, vol. 34, no. 1-2, pp. 146-151, Oct. 2002.

[74] M. Wasser, Z. Bte Osman, and W. Chia, "EAST and Chromator control the destruction and remodeling of muscles during Drosophila metamorphosis," Dev. Biol., vol. 307, no. 2, pp. 380-393, Jul. 2007.

[75] C. Schönbauer et al., "Spalt mediates an evolutionarily conserved switch to fibrillar muscle fate in insects," Nature, vol. 479, no. 7373, pp. 406-409, Nov. 2011.

[76] F. Schnorrer et al., "Systematic genetic analysis of muscle morphogenesis and function in Drosophila," Nature, vol. 464, no. 7286, pp. 287-291, Mar. 2010.

[77] R. Chinta, J. H. Tan, and M. Wasser, "The study of muscle remodeling in Drosophila metamorphosis using in vivo microscopy and bioimage informatics," BMC Bioinformatics, vol. 13 Suppl 17, p. S14, 2012.

[78] D. A. Elliott and A. H. Brand, "The GAL4 system : a versatile system for the expression of genes," Methods Mol. Biol. Clifton NJ, vol. 420, pp. 79-95, 2008.

[79] J. B. Duffy, "GAL4 system in Drosophila: a fly geneticist's Swiss army knife," Genes. N. Y. N 2000, vol. 34, no. 1-2, pp. 1-15, Oct. 2002.

[80] A. Fire, S. Xu, M. K. Montgomery, S. A. Kostas, S. E. Driver, and C. C. Mello, "Potent and specific genetic interference by double-stranded RNA in Caenorhabditis elegans," Nature, vol. 391, no. 6669, pp. 806-811, Feb. 1998.

[81] R. S. Kamath and J. Ahringer, "Genome-wide RNAi screening in Caenorhabditis elegans," Methods San Diego Calif, vol. 30, no. 4, pp. 313-321, Aug. 2003.

[82] J. L. Mummery-Widmer et al., "Genome-wide analysis of Notch signalling in Drosophila by transgenic RNAi," Nature, vol. 458, no. 7241, pp. 987-992, Apr. 2009.

[83] R. A. Neumüller, C. Richter, A. Fischer, M. Novatchkova, K. G. Neumüller, and J. A. Knoblich, "Genome-wide analysis of self-renewal in Drosophila neural stem cells by transgenic RNAi," Cell Stem Cell, vol. 8, no. 5, pp. 580-593, May 2011.

[84] G. Dietzl et al., "A genome-wide transgenic RNAi library for conditional gene inactivation in Drosophila," Nature, vol. 448, no. 7150, pp. 151-156, Jul. 2007.

[85] "The Transgenic RNAi Resource Project." [Online]. Available: http://www.flyrnai.org/TRiP-HOME.html. [Accessed: 24-Sep-2015].

[86] “NIG-Fly - Fly Stocks of National Institute of Genetics -." [Online]. Available: http://www.shigen.nig.ac.jp/fly/nigfly/index.jsp. [Accessed: 24-Sep-2015].

[87] C. A. Schneider, W. S. Rasband, and K. W. Eliceiri, "NIH Image to ImageJ: 25 years of image analysis," Nat. Methods, vol. 9, no. 7, pp. 671-675, Jul. 2012. 
[88] J. Schindelin et al., "Fiji: an open-source platform for biological-image analysis," Nat. Methods, vol. 9, no. 7, pp. 676-682, Jul. 2012.

[89] A. E. Carpenter et al., "CellProfiler: image analysis software for identifying and quantifying cell phenotypes," Genome Biol., vol. 7, no. 10, p. R100, 2006.

[90] P. Kankaanpää et al., "BiolmageXD: an open, general-purpose and highthroughput image-processing platform," Nat. Methods, vol. 9, no. 7, pp. 683-689, Jul. 2012.

[91] W. C. Puah, L. P. Cheok, M. Biro, W. T. Ng, and M. Wasser, "TLM-Converter: reorganization of long time-lapse microscopy datasets for downstream image analysis," BioTechniques, vol. 51, no. 1, Jul. 2011.

[92] "Bio-Formats | LOCI." [Online]. Available: http://loci.wisc.edu/software/bioformats. [Accessed: 04-Oct-2015].

[93] Y. Kuleesha, W. C. Puah, F. Lin, and M. Wasser, "FMAj: a tool for high content analysis of muscle dynamics in Drosophila metamorphosis," BMC Bioinformatics, vol. 15, no. Suppl 16, p. S6, 2014.

[94] S. Osher, Level set methods and dynamic implicit surfaces. New York: Springer, 2003.

[95] “Level Sets - Fiji." [Online]. Available: http://fiji.sc/Level_Sets. [Accessed: 08Jul-2014].

[96] T. Wagner and H.-G. Lipinski, "IJBlob: An ImageJ Library for Connected Component Analysis and Shape Analysis," J. Open Res. Softw., vol. 1, no. 1, Oct. 2013.

[97] B. T. Moore, J. M. Jordan, and L. R. Baugh, "WormSizer: High-throughput Analysis of Nematode Size and Shape," PLoS ONE, vol. 8, no. 2, p. e57142, Feb. 2013.

[98] “JSC Home Page.” [Online]. Available: http://www.jsc.nildram.co.uk/index.htm. [Accessed: 08-Jul-2014].

[99] “JFreeChart." [Online]. Available: http://www.jfree.org/jfreechart/. [Accessed: 08-Aug-2014].

[100] "MySQL :: The world's most popular open source database." [Online]. Available: http://www.mysql.com/. [Accessed: 11-Jul-2014].

[101] "FeatureJ: Laplacian." [Online]. Available: https://imagescience.org/meijering/software/featurej/laplacian/. [Accessed: 09Nov-2017].

[102] T. Ridler, Picture thresholding using an iterative selection method, vol. 8. 1978.

[103] "FeatureJ: Edges." [Online]. Available: https://imagescience.org/meijering/software/featurej/edges/. [Accessed: 09-Nov2017].

[104] P. Soille, Morphological Image Analysis Principles and Applications. Berlin, Heidelberg: Springer Berlin Heidelberg, 2004.

[105] "Morphological Segmentation - Fiji." [Online]. Available: http://fiji.sc/Morphological_Segmentation. [Accessed: 26-Mar-2015].

[106] "Home . ijpb/MorphoLibJ Wiki · GitHub." [Online]. Available: https://github.com/ijpb/MorphoLibJ/wiki. [Accessed: 04-Oct-2015].

[107] "Superpixel code." [Online]. Available: https://www.cs.sfu.ca/ mori/research/superpixels/. [Accessed: 09-Nov-2017].

[108] H. G. Barrow, J. M. Tenenbaum, R. C. Bolles, and H. C. Wolf, "Parametric Correspondence and Chamfer Matching: Two New Techniques for Image Matching," Proc. 5th Int. Jt. Conf. Artif. Intell., vol. 2, pp. 659-663, 1977. 
[109] A. Thayananthan, B. Stenger, P. H. S. Torr, and R. Cipolla, "Shape context and chamfer matching in cluttered scenes," 2003, p. I-127-I-133.

[110] J. Shotton, A. Blake, and R. Cipolla, "Multiscale Categorical Object Recognition Using Contour Fragments," IEEE Trans. Pattern Anal. Mach. Intell., vol. 30, no. 7, pp. 1270-1281, Jul. 2008.

[111] D. T. Nguyen, "A Novel Chamfer Template Matching Method Using Variational Mean Field," 2014, pp. 2425-2432.

[112] N. D. Thanh, W. Li, and P. Ogunbona, "An Improved Template Matching Method for Object Detection," in Computer Vision - ACCV 2009, vol. 5996, H. Zha, R. Taniguchi, and S. Maybank, Eds. Berlin, Heidelberg: Springer Berlin Heidelberg, 2010, pp. 193-202.

[113] S. Beucher, "Watershed, Hierarchical Segmentation and Waterfall Algorithm," in Mathematical Morphology and Its Applications to Image Processing, vol. 2, J. Serra and P. Soille, Eds. Dordrecht: Springer Netherlands, 1994, pp. 69-76.

[114] Y. Kuleesha, W. C. Puah, and M. Wasser, "A model of muscle atrophy based on live microscopy of muscle remodelling in Drosophila metamorphosis," $R$. Soc. Open Sci., vol. 3, no. 2, p. 150517, Feb. 2016.

[115] P. Andrey et al., "Statistical analysis of 3D images detects regular spatial distributions of centromeres and chromocenters in animal and plant nuclei," PLoS Comput. Biol., vol. 6, no. 7, p. e1000853, 2010.

[116] F. He and J. Ma, "A Spatial Point Pattern Analysis in Drosophila Blastoderm Embryos Evaluating the Potential Inheritance of Transcriptional States," PLoS ONE, vol. 8, no. 4, p. e60876, Apr. 2013.

[117] I. Staszewska, I. Fischer, and G. Wiche, "Plectin isoform 1-dependent nuclear docking of desmin networks affects myonuclear architecture and expression of mechanotransducers," Hum. Mol. Genet., vol. 24, no. 25, pp. 7373-7389, Dec. 2015.

[118] "Manual Tracking." [Online]. Available: https://imagej.nih.gov/ij/plugins/track/track.html. [Accessed: 23-Sep-2017].

[119] M. J. Stroud et al., "Nesprin $1 \alpha 2$ is essential for mouse postnatal viability and nuclear positioning in skeletal muscle," J. Cell Biol., vol. 216, no. 7, pp. 19151924, Jul. 2017.

[120] V. Gache, E. R. Gomes, and B. Cadot, "Microtubule motors involved in nuclear movement during skeletal muscle differentiation," Mol. Biol. Cell, vol. 28, no. 7, pp. 865-874, Apr. 2017.

[121] J. A. Ross, A. Pearson, Y. Levy, B. Cardel, C. Handschin, and J. Ochala, "Exploring the Role of PGC-1 $\alpha$ in Defining Nuclear Organisation in Skeletal Muscle Fibres: PGC-1 $\alpha$ AND MYONUCLEAR ORGANISATION," J. Cell. Physiol., vol. 232, no. 6, pp. 1270-1274, Jun. 2017.

[122] M. H. Wilson and E. L. F. Holzbaur, "Nesprins anchor kinesin-1 motors to the nucleus to drive nuclear distribution in muscle cells," Development, vol. 142, no. 1, pp. 218-228, Jan. 2015.

[123] P. Thevenaz, D. Sage, and M. Unser, "Bi-Exponential Edge-Preserving Smoother," IEEE Trans. Image Process., vol. 21, no. 9, pp. 3924-3936, Sep. 2012.

[124] "Mexican Hat Filter." [Online]. Available: http://rsb.info.nih.gov/ij/plugins/mexican-hat/index.html. [Accessed: 04-Oct2015]. 
[125] Y. Al-Kofahi, W. Lassoued, W. Lee, and B. Roysam, "Improved Automatic Detection and Segmentation of Cell Nuclei in Histopathology Images," IEEE Trans. Biomed. Eng., vol. 57, no. 4, pp. 841-852, Apr. 2010.

[126] H. Irshad, A. Veillard, L. Roux, and D. Racoceanu, "Methods for Nuclei Detection, Segmentation, and Classification in Digital Histopathology: A Review\&amp;\#x2014;Current Status and Future Potential," IEEE Rev. Biomed. Eng., vol. 7, pp. 97-114, 2014.

[127] L. Chen, L. Chan, Z. Zhao, and H. Yan, "A novel cell nuclei segmentation method for 3D C. elegans embryonic time-lapse images," BMC Bioinformatics, vol. 14, no. 1 , p. 328, 2013.

[128] "Hull And Circle." [Online]. Available: http://rsb.info.nih.gov/ij/plugins/hullcircle.html. [Accessed: 03-Oct-2015].

[129] "Segmentation Editor - Fiji." [Online]. Available: http://fiji.sc/Segmentation_Editor. [Accessed: 26-Mar-2015].

[130] “ij-plugins - IJ Plugins: k-means Clustering." [Online]. Available: http://ijplugins.sourceforge.net/plugins/segmentation/k-means.html. [Accessed: 09Nov-2017].

[131] “MTrack2,” ImageJ. [Online]. Available: http://imagej.net/MTrack2. [Accessed: 23-Sep-2017].

[132] I. Ribeiro, L. Yuan, G. Tanentzapf, J. J. Dowling, and A. Kiger, "Phosphoinositide Regulation of Integrin Trafficking Required for Muscle Attachment and Maintenance," PLoS Genet., vol. 7, no. 2, p. e1001295, Feb. 2011.

[133] S. M. Gorski et al., "A SAGE approach to discovery of genes involved in autophagic cell death," Curr. Biol. CB, vol. 13, no. 4, pp. 358-363, Feb. 2003.

[134] C.-Y. Lee, E. A. Clough, P. Yellon, T. M. Teslovich, D. A. Stephan, and E. H. Baehrecke, "Genome-Wide Analyses of Steroid- and Radiation-Triggered Programmed Cell Death in Drosophila," Curr. Biol., vol. 13, no. 4, pp. 350-357, Feb. 2003.

[135] H.-W. Tang, H.-M. Liao, W.-H. Peng, H.-R. Lin, C.-H. Chen, and G.-C. Chen, "Atg9 Interacts with dTRAF2/TRAF6 to Regulate Oxidative Stress-Induced JNK Activation and Autophagy Induction," Dev. Cell, vol. 27, no. 5, pp. 489-503, Dec. 2013.

[136] P. Nagy, K. Hegedűs, K. Pircs, Á. Varga, and G. Juhász, "Different effects of Atg2 and Atg18 mutations on Atg8a and Atg9 trafficking during starvation in Drosophila," FEBS Lett., vol. 588, no. 3, pp. 408-413, Jan. 2014.

[137] D. L. Berry and E. H. Baehrecke, "Growth arrest and autophagy are required for salivary gland cell degradation in Drosophila," Cell, vol. 131, no. 6, pp. 11371148, Dec. 2007.

[138] D. Denton et al., "Relationship between growth arrest and autophagy in midgut programmed cell death in Drosophila," Cell Death Differ., vol. 19, no. 8, pp. 1299-1307, Aug. 2012.

[139] P. Nagy et al., "Atg17/FIP200 localizes to perilysosomal Ref(2)P aggregates and promotes autophagy by activation of Atg1 in Drosophila," Autophagy, vol. 10, no. 3, pp. 453-467, Mar. 2014.

[140] M. Kim et al., "Drosophila Fip200 is an essential regulator of autophagy that attenuates both growth and aging," Autophagy, vol. 9, no. 8, pp. 1201-1213, Aug. 2013. 
[141] J. S. Karres, V. Hilgers, I. Carrera, J. Treisman, and S. M. Cohen, "The Conserved microRNA MiR-8 Tunes Atrophin Levels to Prevent Neurodegeneration in Drosophila," Cell, vol. 131, no. 1, pp. 136-145, Oct. 2007.

[142] M. L. Bland, R. J. Lee, J. M. Magallanes, J. K. Foskett, and M. J. Birnbaum, "AMPK supports growth in Drosophila by regulating muscle activity and nutrient uptake in the gut," Dev. Biol., vol. 344, no. 1, pp. 293-303, Aug. 2010.

[143] K. I. Kimura and J. W. Truman, "Postmetamorphic cell death in the nervous and muscular systems of Drosophila melanogaster," J. Neurosci. Off. J. Soc. Neurosci., vol. 10, no. 2, pp. 403-401, Feb. 1990.

[144] D. M. Gwinn et al., "AMPK Phosphorylation of Raptor Mediates a Metabolic Checkpoint," Mol. Cell, vol. 30, no. 2, pp. 214-226, Apr. 2008.

[145] D. Meley et al., "AMP-activated protein kinase and the regulation of autophagic proteolysis," J. Biol. Chem., vol. 281, no. 46, pp. 34870-34879, Nov. 2006.

[146] C. Behrends, M. E. Sowa, S. P. Gygi, and J. W. Harper, "Network organization of the human autophagy system," Nature, vol. 466, no. 7302, pp. 68-76, Jul. 2010.

[147] A. Erkner et al., "Grunge, related to human Atrophin-like proteins, has multiple functions in Drosophila development," Dev. Camb. Engl., vol. 129, no. 5, pp. 1119-1129, Mar. 2002.

[148] D. N. Martin et al., "Proteomic analysis of steroid-triggered autophagic programmed cell death during Drosophila development," Cell Death Differ., Jan. 2007.

[149] R. Farkaš et al., "Vacuole dynamics in the salivary glands of Drosophila melanogaster during prepupal development," Dev. Growth Differ., vol. 57, no. 1, pp. 74-96, Jan. 2015.

[150] J. Zirin, J. Nieuwenhuis, and N. Perrimon, "Role of Autophagy in Glycogen Breakdown and Its Relevance to Chloroquine Myopathy," PLoS Biol., vol. 11, no. 11, p. e1001708, Nov. 2013. 